\title{
ANALYSIS AND CONTROL OF INVERTER OPERATION IN MICROGRIDS
}

PENG XIAOYANG

School of Electrical and Electronic Engineering

A thesis submitted to the Nanyang Technological University in fulfilment of the requirement for the degree of

Doctor of Philosophy 


\section{ACKNOWLEDGEMENT}

The completion of this dissertation cannot be achieved without the assistance of several individuals who gave me guidance and valuable advice in the process of my research and my thesis writing.

I would like to express my deepest appreciation of my supervisor, Associate Professor So Ping Lam, whose generous guidance and support never stop throughout my whole research period. His knowledge, dedication and keen interest in power systems, distributed generation and microgrids have affected me deeply for my research. His meticulous scrutiny, broad research vision and timely advice have helped me to a great extent to accomplish this research.

I am grateful to Dr. Tan Kuan Tak for his enormous assistance and guidance. His prompt inspiration, scholarly suggestion, and enthusiastic attitude have encouraged me to work hard on my research. His academic guidance and constructive criticisms are extremely valuable for clearing the doubts within me.

I would also like to thank Mr. Li Chao, Mr. Wang Benfei and Mr. Gelbert Lariosa for their academic support and kind help in life throughout the years of my research. Their support keeps me working until the completion of this thesis.

I am thankful for Nanyang Technological University, Singapore for giving me an opportunity to pursue my graduate study with a scholarship. I am also grateful for all my friends and related staff from Wuhan University, China for the memory of my undergraduate years.

Finally, I would like to thank my parents for their endless love and support, for their teaching and attitude. I owe everything to them and I wish that this completed thesis could make them proud one more time. 


\section{SUMMARY}

The energy crisis boosts the integration of renewable energy resources (RESs) worldwide. To develop the operation and control strategies to integrate RESs, microgrid concept is proposed and studied. The distributed generation (DG) units integrated in microgrids are usually connected through DG inverters.

This thesis conducts analysis, and proposes and investigates inverter operation and control system on several different AC and DC microgrids.

The coordination and control of the inverter operation in microgrids is crucial to integrate different RESs in power systems. This thesis investigates different problems in the microgrid to accommodate multiple inverters while sustaining the stability and reliability of power systems.

Firstly, a centralized control strategy incorporated in an energy management system (EMS) is proposed for an AC based microgrid consisting of Photovoltaic (PV) panels, battery, fuel cell and microturbine. For this inverter-interfaced AC microgrid, parallel operation of all the inverters during both grid-connected and islanded operations is realized through a coordinated control in the EMS with two different model predictive control (MPC) based control modes for inverters: current control mode (CCM) and voltage control mode (VCM). Several case studies are carried out through simulations with detailed analysis, and the results validate that the proposed centralized control strategy can handle different operations in both grid-connected and islanded conditions.

Secondly, the system frequency analysis of the islanded condition of an inverterinterfaced AC microgrid is conducted. A combined centralized and decentralized control strategy is proposed using MPC based inverter control. A frequency model is incorporated in the EMS for coordinated control among different DG units. Various test scenarios are studied through simulations. These simulations illustrate the frequency problems and verify that the proposed combined centralized and decentralized control strategy can handle different operations in islanded condition. 
Thirdly, the analysis of inverter operation and control is extended to a DC microgrid to harness the excess wind energy in a poultry farm. A double-inverter configuration between DC bus and AC bus in the DC microgrid is proposed. A double-mode MPC algorithm (VCM and CCM) and a control strategy in the EMS are proposed to handle the two inverters' operation. Several simulation tests are analyzed, and the test results demonstrate the effectiveness of the operation and control system and the design concept of the double-inverter operation. 


\section{TABLE OF CONTENTS}

ACKNOWLEDGEMENT

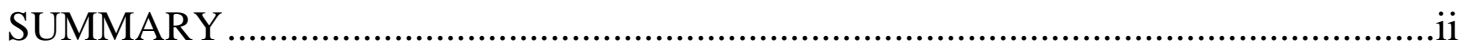

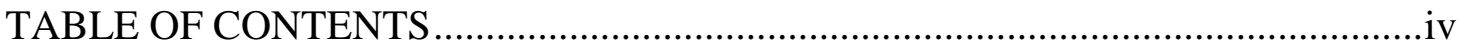

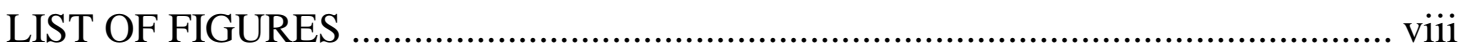

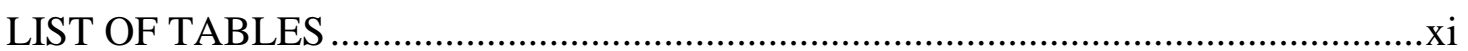

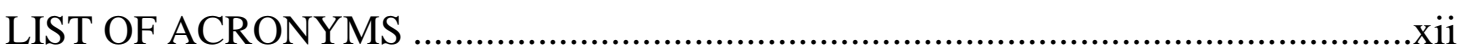

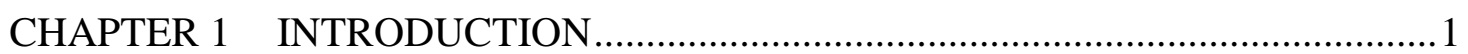

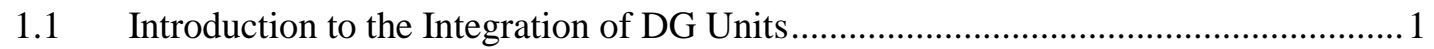

1.1.1 Integration of Photovoltaic (PV) Power Generation ......................................... 2

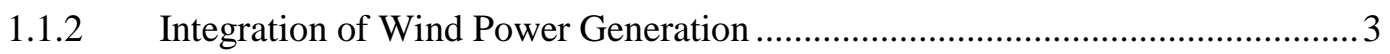

1.1.3 Integration of Energy Storage System (ESS) ……..........................................5

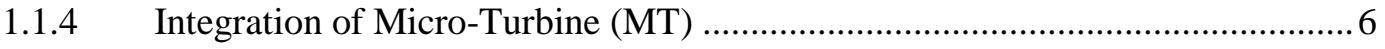

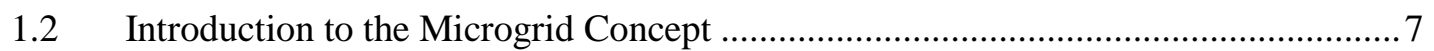

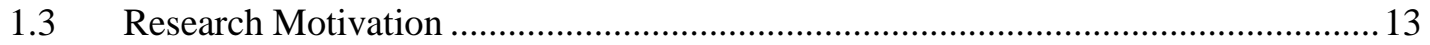

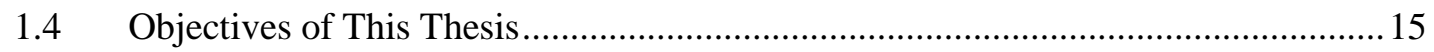

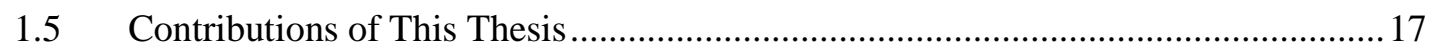

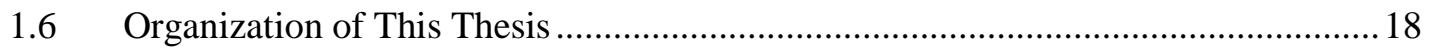

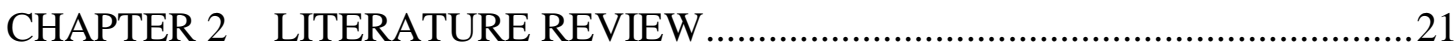

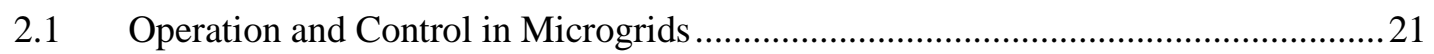

2.1.1 Centralized and Decentralized Approaches .................................................2 21

2.1.2 Operation and Control of Grid-Connected and Islanded Microgrids................224

2.2 Real Power Control and Management in Microgrids.............................................26

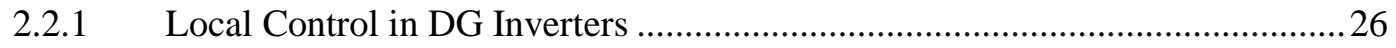

2.2.2 Real Power Management in Microgrid EMS ……….....................................2. 29

2.3 Reactive Power Compensation in Microgrids.......................................................... 31

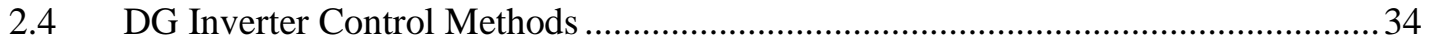




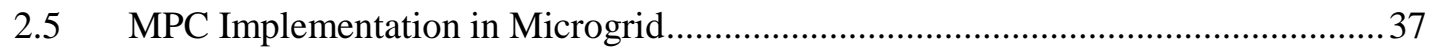

2.6 Operation and Control Strategy in Microgrid EMS …............................................. 41

2.6.1 Operation and Control Strategy Diagram.......................................................... 42

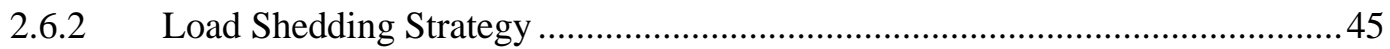

2.7 Network Configuration in DC Based Microgrids ................................................... 47

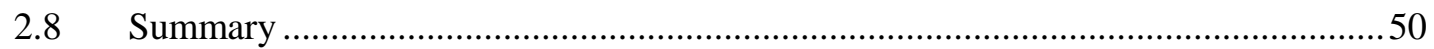

CHAPTER 3 CENTRALIZED CONTROL FOR PARALLEL OPERATION OF DISTRIBUTED GENERATION INVERTERS IN MICROGRIDS [111].................52

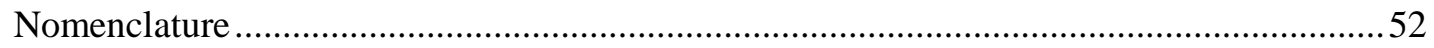

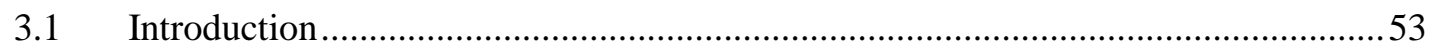

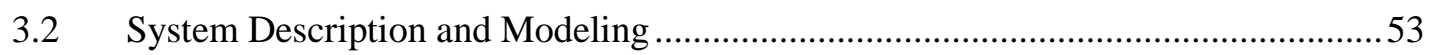

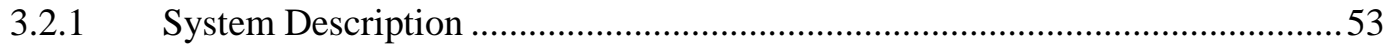

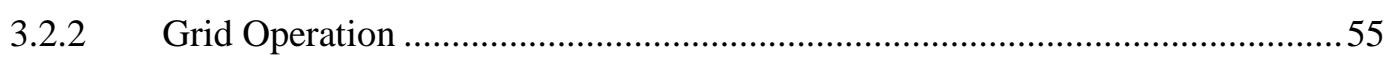

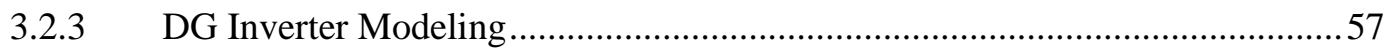

3.2.4 Droop Control for Parallel Operation of Inverters ............................................. 61

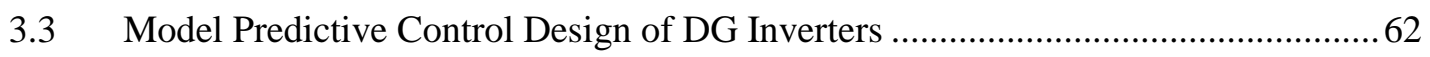

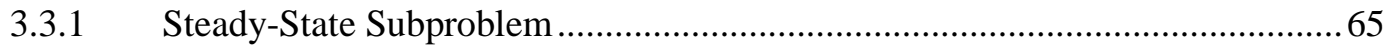

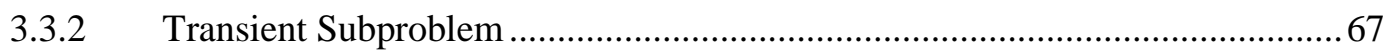

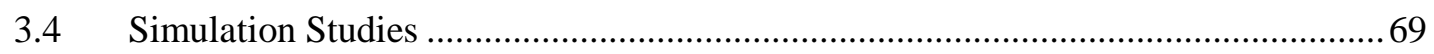

3.4.1 Test Case 1: Power Quality Improvement with Load Sharing During Grid-

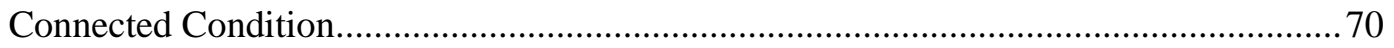

3.4.2 Test Case 2: Load Sharing during Islanded condition with Droop Control .......74

3.4.3 Test Case 3: Investigation on Network Voltage Changes .................................77

3.4.4 Test Case 4: Investigation on Increased Fault Level........................................ 78

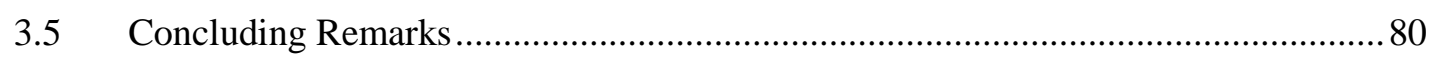

CHAPTER 4 Operation and Control of Distributed Generation Inverters in an

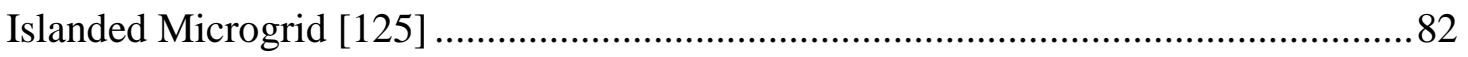

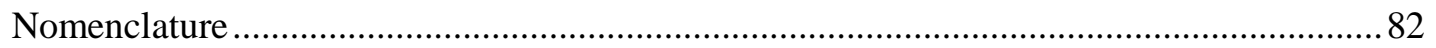

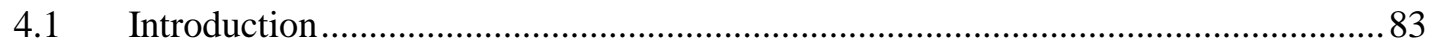




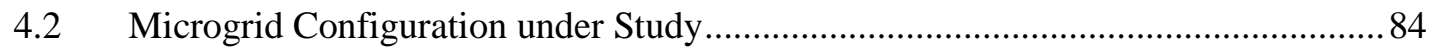

4.3 Modeling and Control of Frequency in Microgrid during Islanded Condition .......... 85

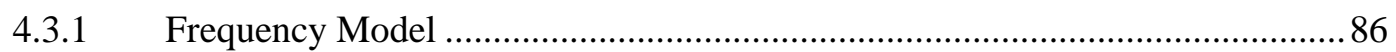

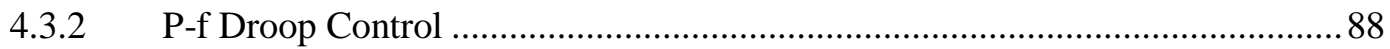

4.3.3 Modified Secondary Frequency Control .......................................................... 89

4.4 Proposed MPC Algorithm for DG Inverters ......................................................... 90

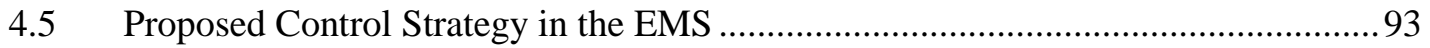

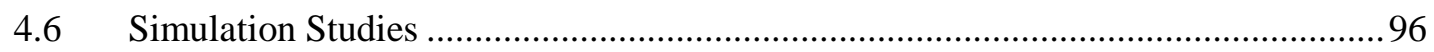

4.6.1 Test Case 1: Islanding during Light Load Condition .......................................97

4.6.2 Test Case 2: Islanding during Heavy Load Condition ....................................101

4.6.3 Test Case 3: Comparison between MPC and PR in SB discharging................ 104

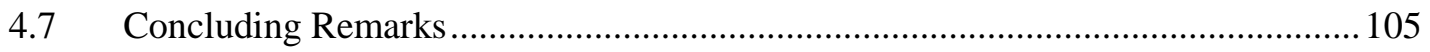

CHAPTER 5 Control and Operation of a DC Grid Based Wind Power Generation

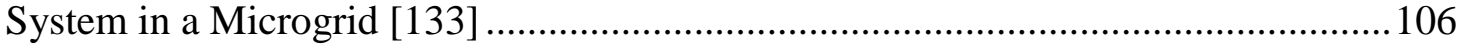

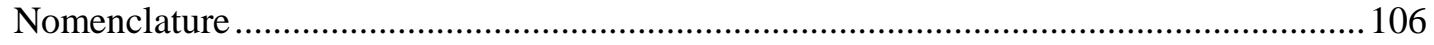

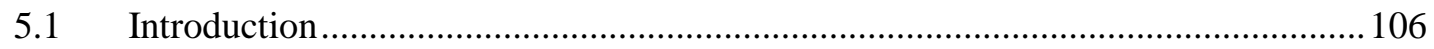

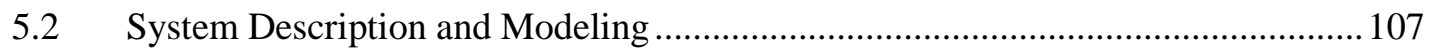

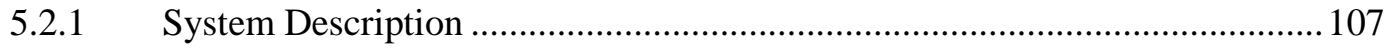

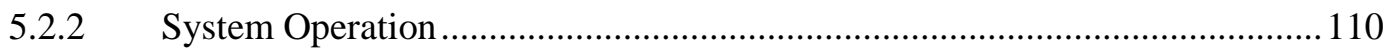

5.2.3 AC/DC Rectifier Modeling ....................................................................... 110

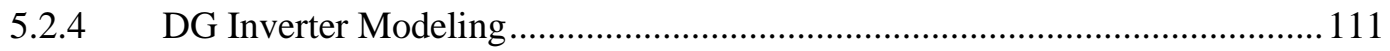

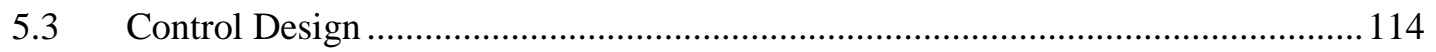

5.3.1 Control Design for the AC/DC Rectifier........................................................ 114

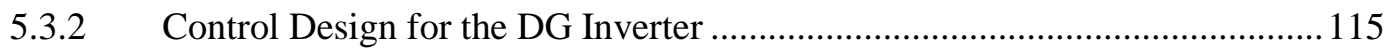

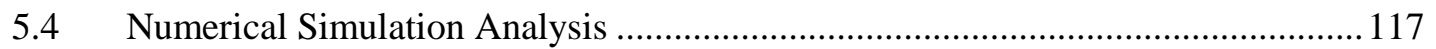

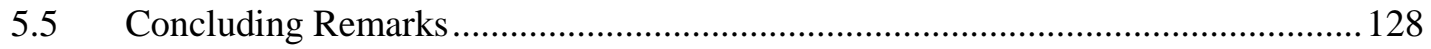

CHAPTER 6 CONCLUSION AND RECOMMENDATIONS ............................ 129

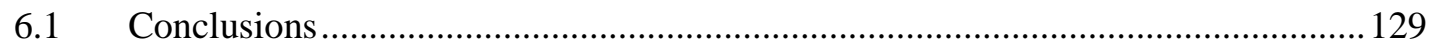

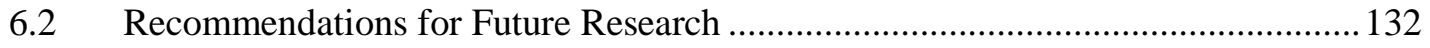




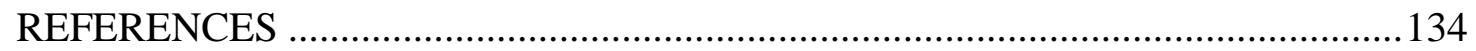

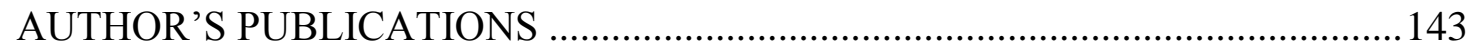




\section{LIST OF FIGURES}

Figure 1-1: A solar irradiance sample on the rooftop inside the NTU campus. .............3

Figure 1-2: Integration of wind power generation through a DC network. ...................4

Figure 1-3: A schematic diagram of an MT connecting to grid and load...................... 7

Figure 1-4: (a) An AC microgrid with PV panels, WT and SB; (b) a DC microgrid

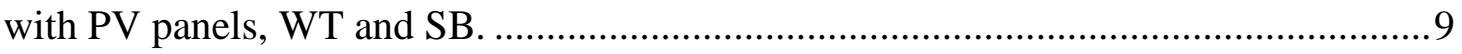

Figure 1-5: An example of the operation and control structure in a microgrid .............11

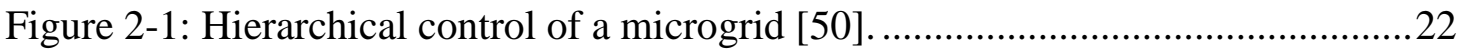

Figure 2-2: General block diagram of the dynamic LFC .........................................2

Figure 2-3: Real power versus frequency (P-f) droop characteristics. ........................28

Figure 2-4: Control strategy proposed in the EMS [71] ..........................................29

Figure 2-5: Control strategy proposed in the microgrid EMS [72] .............................30

Figure 2-6: Reactive power versus voltage (Q-v) droop characteristics......................33

Figure 2-7: General PI control configuration in the dq0 frame [83] ...........................35

Figure 2-8: General PR control configuration in the $\alpha \beta \gamma$ frame [83]. .........................36

Figure 2-9: A general three-phase DG inverter configuration with RL load [93].......40

Figure 2-10: Operation and control strategy proposed in [97]. .................................43

Figure 2-11: Operation and control strategy for a PV/battery system [98] .................44

Figure 2-12: Operation and control strategy proposed in [70]. ..................................44

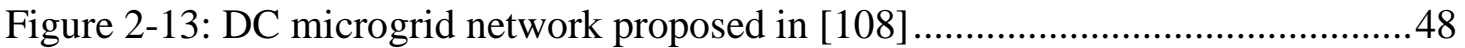

Figure 2-14: Hybrid DC/AC microgrid configuration proposed in [110] ...................49

Figure 3-1: Overall configuration of the proposed microgrid architecture...................54

Figure 3-2: Operation of the SB during grid-connected condition. ..............................56

Figure 3-3: Operation of the SB and PEMFC stack during islanded condition.............57

Figure 3-4: Equivalent single-phase representation of the DG inverter of MT $(j=1)$ or PV array $(j=2)$ during grid-connected condition.................................................57

Figure 3-5: Equivalent single-phase representation of the DG inverters of the $S B(j=3)$ and the PEMFC stack $(\mathrm{j}=4)$ during islanded condition.............................................58

Figure 3-6: Single-phase representation of the DG inverter during both grid-connected

Figure 3-7: Overall MPC controller for the DG inverter with E/KF denoting the exogenous Kalman filter and P/KF denoting the plant Kalman filter. 
Figure 3-8: Waveforms of three-phase Load 1 current $i_{\mathrm{L} 1}$ (top), three-phase DG 1 current $\mathrm{i}_{\mathrm{DG} 1}$ (middle), and three-phase grid current $\mathrm{i}_{\mathrm{G} 1}$ (bottom) ..............................71

Figure 3-9: Waveforms of grid voltage $v_{G}$ and grid current $i_{G 1}$ for phase a. ...............72

Figure 3-10: Real (top) and reactive (bottom) power delivered by DG inverter 1......73

Figure 3-11: Real (top) and reactive (bottom) power delivered by DG inverter 2 ......73

Figure 3-12: Total real (top) and reactive (bottom) power delivered by the grid to

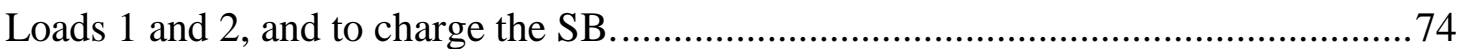

Figure 3-13: Real (top) and reactive (bottom) power delivered by DG inverter 1.......75

Figure 3-14: Real (top) and reactive (bottom) power delivered by DG inverter 2 .......75

Figure 3-15: Real power delivered by DG inverter 3 ............................................. 76

Figure 3-16: Real power delivered by DG inverter 4 ......................................... 76

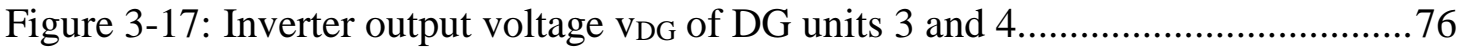

Figure 3-18: Voltage profile (in p.u.) for changes in the real and reactive power of the

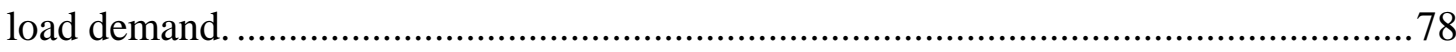

Figure 3-19: Equivalent single-line diagram of the proposed microgrid during fault analysis

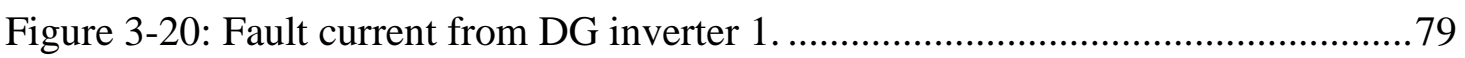

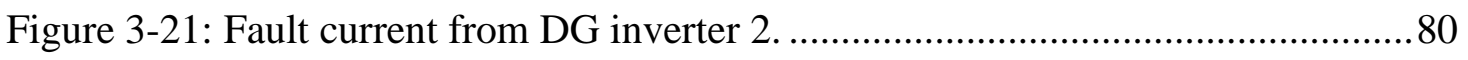

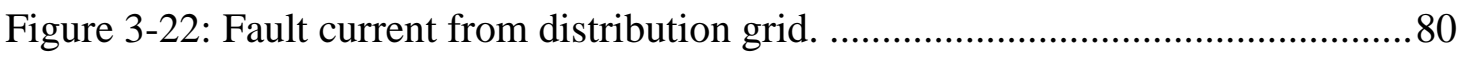

Figure 4-1: Proposed microgrid configuration under study.....................................84

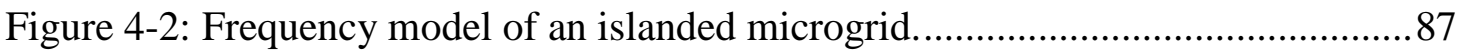

Figure 4-3: Relationship between frequency and real power: (a) without frequency

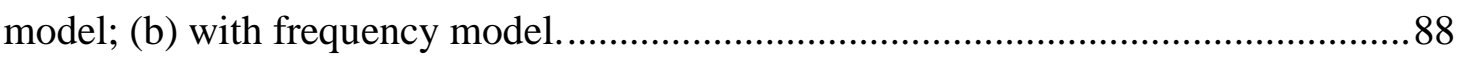

Figure 4-4: Three-phase DG inverter model........................................................... 91

Figure 4-5: Proposed control strategy adopted by the EMS .....................................94

Figure 4-6: Real power delivered by the grid and PV in Test Case 1. ........................98

Figure 4-7: Reactive power delivered by the PV in Test Case 1..............................98

Figure 4-8: Real power delivered by the SB and MT in Test Case 1........................98

Figure 4-9: Frequency of the microgrid in Test Case 1............................................99

Figure 4-10: RMS value of the PCC voltage in Test Case 1....................................99

Figure 4-11: Real power delivered by the grid and PV, and load demand in Test Case 2. 101

Figure 4-12: Reactive power delivered by the PV in Test Case 2............................102

Figure 4-13: Real power delivered by the SB and MT in Test Case 2. ......................102 
Figure 4-14: Frequency of the microgrid in Test Case 2.

Figure 4-15: Comparison between MPC and PR in real power delivered by the SB in Test Case 3.

Figure 5-1: Overall configuration of the proposed DC grid based wind power generation system in a microgrid. .108

Figure 5-2: Power circuit of a PMSG connected to an AC/DC voltage source rectifier.

Figure 5-3: Single-phase representation of the three-phase DG inverter. 112

Figure 5-4: Configuration of the proposed controller for the AC/DC converter. 115

Figure 5-5: Real (top) and reactive (bottom) power delivered by inverter 1 119

Figure 5-6: Real (top) and reactive (bottom) power delivered by inverter 2 . 120

Figure 5-7: Real (top) and reactive (bottom) power delivered by the grid.... 120

Figure 5-8: Real (top) and reactive (bottom) power consumed by the loads 121

Figure 5-9: DC grid voltage. 122

Figure 5-10: Real (top) and reactive (bottom) power delivered by inverter 1 . 123

Figure 5-11: Real (top) and reactive (bottom) power delivered by inverter 2............ 123

Figure 5-12: Real (top) and reactive (bottom) power delivered by the grid................ 124

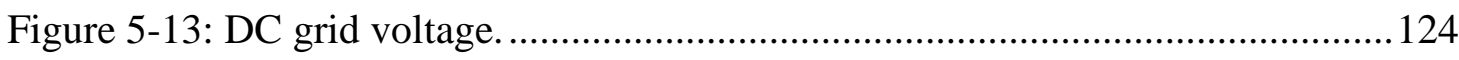

Figure 5-14: Real (top) and reactive (bottom) power delivered by the grid................ 126

Figure 5-15: Real (top) and reactive (bottom) power delivered by inverter 1........... 126

Figure 5-16: Real (top) and reactive (bottom) power delivered by inverter 2........... 127

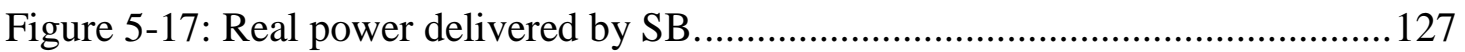

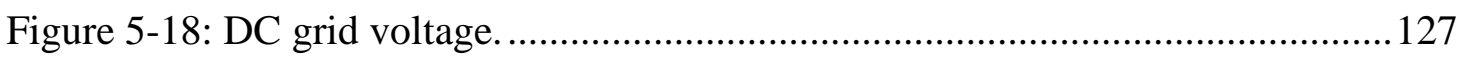




\section{LIST OF TABLES}

Table 2-1: Israel Electric Corporation Under-Frequency Load Shedding Scheme [102]

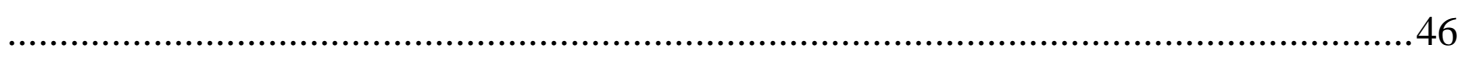

Table 3-1: Parameters of the Proposed System ......................................................... 70

Table 3-2: Numerical Values of Currents and Voltages during Fault ..........................79

Table 4-1: Parameters Used in the Simulation Studies.............................................96

Table 5-1: Parameters of the Proposed System ........................................................... 118 


\section{LIST OF ACRONYMS}

\section{List of Acronyms}

\begin{tabular}{|c|c|c|}
\hline $\mathrm{AC}$ & - & Alternating Current \\
\hline $\mathrm{AGC}$ & - & Automatic Generation Control \\
\hline BESS & - & Battery Energy Storage System \\
\hline BEMS & - & Battery Energy Management System \\
\hline $\mathrm{CB}$ & - & Circuit Breaker \\
\hline $\mathrm{CCM}$ & - & Current Control Mode \\
\hline DG & - & Distributed Generation \\
\hline $\mathrm{DC}$ & - & Direct Current \\
\hline DOE & - & Department of Energy \\
\hline DMS & - & Distributed Management System \\
\hline DSEM & 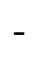 & Distribution State Estimator Model \\
\hline DSM & - & Demand Side Management \\
\hline ECM & - & Event Calculator Module \\
\hline EDB & 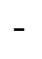 & Economic Development Board \\
\hline EMS & - & Energy Management System \\
\hline ESS & - & Energy Storage System \\
\hline $\mathrm{FCM}$ & - & Frequency Calculator Module \\
\hline FCS & - & Finite Control Set \\
\hline LFC & - & Load Frequency Control \\
\hline LPF & - & Low-Pass Filter \\
\hline LSCM & - & Load Shedding Controller Module \\
\hline LTI & - & Linear Time-Invariant \\
\hline LV & - & Low Voltage \\
\hline MAS & - & Multi-Agent System \\
\hline $\mathrm{MC}$ & - & Microsource Controller \\
\hline MGCC & 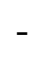 & Microgrid Central Controller \\
\hline MPC & - & Model Predictive Control \\
\hline MPPT & - & Maximum Power Point Tracking \\
\hline MT & - & Micro-Turbine \\
\hline
\end{tabular}




$\begin{array}{lll}\text { MV } & - & \text { Medium Voltage } \\ \text { NEA } & - & \text { National Environment Agency } \\ \text { NTU } & - & \text { Nanyang Technological University } \\ \text { OPF } & - & \text { Optimal Power Flow } \\ \text { PCC } & - & \text { Point of Common Coupling } \\ \text { PEMFC } & - & \text { Proton Exchange Membrane Fuel Cell } \\ \text { PEV } & - & \text { Plug-in Electric Vehicle } \\ \text { PID } & - & \text { Proportional Integral Derivative } \\ \text { PMSG } & - & \text { Permanent Magnet Synchronous Generator } \\ \text { PR } & - & \text { Proportional-Resonant } \\ \text { PV } & - & \text { Photovoltaic } \\ \text { PWM } & - & \text { Pulse-Width-Modulation } \\ \text { REIDS } & - & \text { Renewable Energy Integration Demonstrator-Singapore } \\ \text { RES } & - & \text { Renewable Energy Source } \\ \text { RMS } & - & \text { Root Mean Square } \\ \text { R\&D } & - & \text { Research and Development } \\ \text { SB } & - & \text { Storage Battery } \\ \text { SOC } & - & \text { State of Charge } \\ \text { STATCOM } & - & \text { Static Synchronous Compensator } \\ \text { SVC } & - & \text { Static VAr Compensator } \\ \text { THD } & - & \text { Total Harmonic Distortion } \\ \text { TOU } & - & \text { Time-of-Use } \\ \text { US } & - & \text { United States } \\ \text { VCM } & - & \text { Voltage Control Mode } \\ \text { WBM } & - & \text { Wind Turbine/Battery Mode } \\ \text { WDBM } & - & \text { Wind Turbine/Diesel Generator/Battery Mode } \\ \text { WG } & - & \text { Wind Generator } \\ \text { WT } & - & -\end{array}$




\section{CHAPTER 1 INTRODUCTION}

This chapter presents a general introduction to the integration of different distributed generation (DG) units, the characteristics, the operation and control of microgrids. The motivation, objectives, contributions and organization of the thesis are also discussed.

\subsection{Introduction to the Integration of DG Units}

The energy problem is one of the biggest problems which the world has encountered. As the energy consumption of the entire world continues to increase, relying on traditional fossil fuels is unpractical and unsustainable. Renewable energy sources (RESs) are becoming more popular these days because of this energy problem. More and more countries are promoting RESs to satisfy their rapidly-increasing energy consumptions. According to [1], the percentage of RESs' penetration in the total global energy generation increased from $2.4 \%$ (2014) to $2.8 \%$ (2015), and RESs' penetration in Singapore increased $7.8 \%$ from 2014 to 2015. From this data, the energy production of RESs is steadily increasing.

For power systems, RESs such as solar and wind energy are usually harnessed in distributed areas, which is different from the situations of traditional power plants. Such power generations, which are integrated within power distribution systems, are defined as DG units. It is universally acknowledged that most of the RESs are utilized as DG units [2]. Aside from supplying power to power systems, DG units also have many other advantages [3], [4]:

- Less transmission loss

- Less construction time

- Low investment risk

- Possibility to operate in islanded mode

However, with the current technology, high penetration of DG nits in power systems are technically viable, as there are a few examples such as in Texas U. S., California U. S., Denmark and Spain. 


\subsubsection{Integration of Photovoltaic (PV) Power Generation}

Research works about utilizing PV power generation can be found as early as 1839 when French scientist Edmond Becquerel discovered the photovoltaic effect while experimenting with an electrolytic cell comprised of two metal electrodes placed in an electricity-conducting solution [5]. Since the power transmitted from the sun can be regarded as eternal compared to a human's lifespan, PV power generation technology has grown rapidly through the years of development. This development is not only reflected in the increased efficiency of the power conversion from solar energy to electrical energy, it is also reflected in the continuously increasing penetration of PV power generation in power systems around the world [6], [7].

However, even though the penetration of PV power generation increased in the past few decades, the percentage of PV power generation in total power generation from all sources still remains as low as 1 2\% worldwide [1]. The reasons why it is difficult to integrate a significant amount of PV power generation are mainly because of night time zero generation and system stability issues due to its intermittent nature.

PV power generation is directly linked to solar irradiance where the PV panels are installed. It is obvious that PV power generation cannot operate during the night because there is no sun. This means that PV power generation cannot generate power consistently throughout the day and night. Therefore, if PV power generation is to make a large penetration in the power system, possible solutions need to be proposed to meet nighttime power consumption. Many research works [8], [9] have been analyzing how to compensate for the power generation at night when PV power generation is zero. Most of the research works proposed the use of energy storage systems (ESSs) which can store energy during the period when the generation is larger than the demand [9]. Since night time is a rather long period, the most important factors are the capacity of the ESSs and the energy management issue. These types of problems can be solved ultimately by providing ESSs with excessive capacities when the price of the ESS is reduced to such a level that installing such large ESSs becomes economically viable. 
Additionally, the solar irradiance can also change significantly during the day. Figure 1-1 shows the solar irradiance detected from a PV panel on the rooftop of the School of Electrical and Electronic Engineering in Nanyang Technological University (NTU). From Figure 1-1, the variations in solar irradiance are quite significant. Since the output from PV power generation is proportional to the solar irradiance on the PV panels [10], it is inevitable that PV power generation will fluctuate under such an unstable solar irradiance.

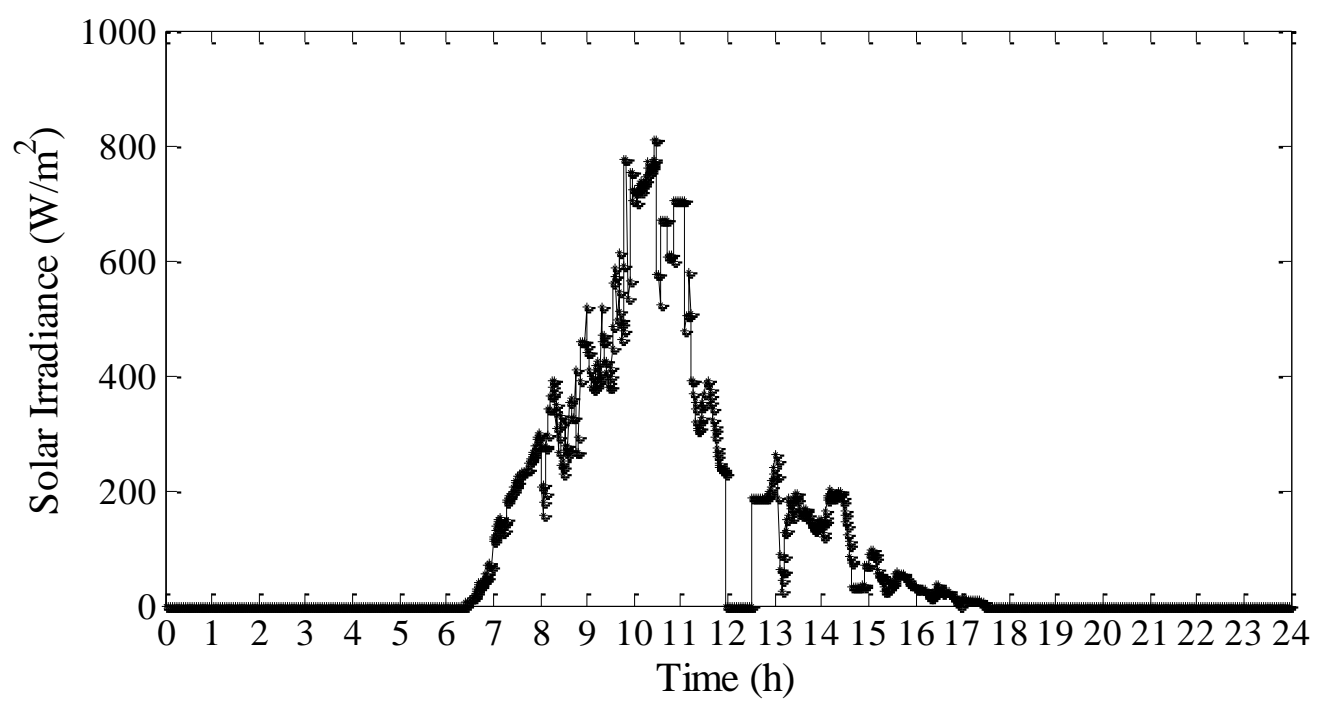

Figure 1-1: A solar irradiance sample on the rooftop inside the NTU campus.

\subsubsection{Integration of Wind Power Generation}

As another RES, wind energy is also very promising to be integrated into power systems with high penetration rate. Since the first power generating windmill, built by James Blyth in 1887 [11], wind power generation has developed for over a century. New types of wind power generation are being discovered and investigated around the world. Offshore wind power generation [12] is now very popular in research facilities and universities, and all these developments show us a bright future for wind power generation.

However, many problems occur during the integration of wind power generation. Like PV power generation, wind power generation can also be greatly affected by external 
environmental conditions such as wind speed, wind direction, etc. Solutions to these kinds of problems are already implemented in industrial wind turbines (WTs). The WTs nowadays can track the wind direction and maintain a constant speed when the wind speed varies.

Apart from external condition problems, grid integration problems are complicated and challenging as well. The direct output from wind power generation is usually in the form of low level AC power. To supply power to a power system, the WTs are usually connected to the distribution network through a rectifier and an inverter. However, if one WT uses one rectifier and one inverter to operate normally, it will be very expensive to increase the penetration of wind power generation. Therefore, researchers discovered that introducing a DC network in the integration process of wind power generation [12] can greatly decrease the number of inverters which are needed. Figure 1-2 shows the integration of wind power generation through a DC network.

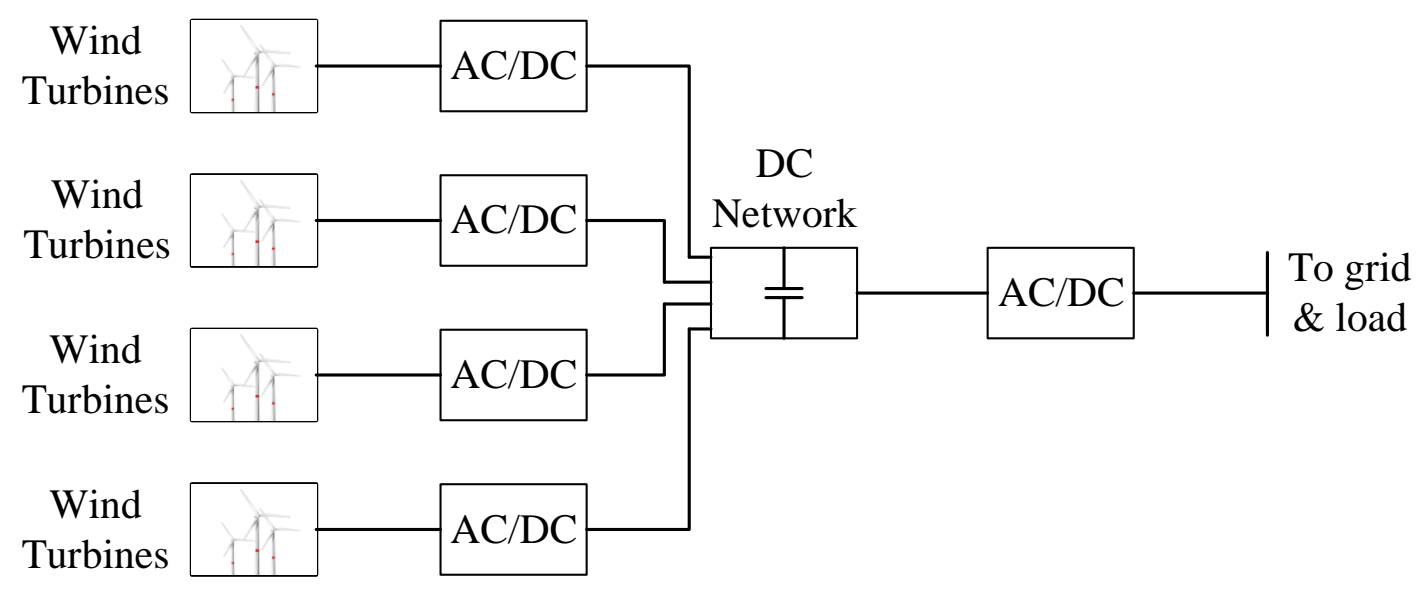

Figure 1-2: Integration of wind power generation through a DC network.

From Figure 1-2, this kind of network configuration will reduce the use of inverters. One of the biggest advantages of this configuration is that it reduces the installation and maintenance costs. With such a configuration, large penetration of wind power generation becomes more realistic and economical. However, this configuration will also bring stability problems to the operation of the distribution network. Since the number of inverters is reduced, the operation of the inverters is critical to wind power generation. As shown in Figure 1-2, when a technical failure occurs in the inverter 
between the DC network and the grid, the distribution network will suddenly lose all the WTs. If the wind power penetration in the distribution network is relatively high, a blackout may happen when the energy management system (EMS) in the distribution network cannot solve the power imbalance problem.

\subsubsection{Integration of Energy Storage System (ESS)}

Apart from RESs, there are many other types of DG units. The most commonly used DG units in a microgrid, other than RESs, are ESSs.

ESSs in power systems refer to devices which store energy that can be used to supply electrical power to power systems under certain circumstances (e.g., power imbalance [13], reactive power compensation [14], etc.). There are many different types of ESSs found in related research works, such as pumped hydro ESS [15], flywheel ESS [16], and battery ESS (BESS) [17]. Among these different types of ESSs, BESS is the most commonly used one in recent research works.

BESS utilizes a storage battery (SB) to store electrical energy, which can be supplied directly to the distribution system through a DC/DC converter and an inverter [9]. Because an SB can generate power within a short response time as it is a power electronic device, SB is widely used in the research of microgrids to solve some stability problems [18]. However, there are some technical issues associated with BESS which must be addressed.

The energy capacity of an SB should be fixed before installation, which means that the SB cannot generate power infinitely. Therefore, it is very important to implement a battery energy management system (BEMS) to control the charging and discharging of the SB. Managing the state of charge (SOC) of an SB using various methods [19], [20] can reduce the required capacity of the $\mathrm{SB}$, reduce charging cost and sustain normal operation of the microgrid.

Apart from the energy management problem mentioned above which has been investigated for a long time, the control algorithms for the inverter which are used by 
most of the research works have not changed much in the past few decades. Proportional integral derivative (PID) control algorithm and proportional-resonant (PR) control algorithm are the two main algorithms which are widely used for inverter control. For these control algorithms, tuning of gains (i.e., proportional gain, integral gain, etc.) is necessary and very crucial because the response time and the system stability greatly rely on the selection of the gains. In addition, it is difficult to implement any constraints in the control since constraints are not supported naturally. Besides, these controllers can only work together with pulse-width-modulation (PWM) generators, which makes the control system complicated and expensive. Compared to the energy management problem, the control algorithm problem has a direct impact on the system stability. Therefore, it would be more meaningful to study and investigate the control algorithm for the inverter.

\subsubsection{Integration of Micro-Turbine (MT)}

Micro-turbines (MTs) are small combustion turbines which have the power capacity of $25 \mathrm{~kW}$ to $1 \mathrm{MW}$ [21]. Different MTs use different types of fuels in the combustion chamber. Mostly, the commercial MTs nowadays use non-renewable energy sources, such as natural gas and coal. The energy efficiency of an MT commonly ranges from $20 \%$ to the low $30 \%$ range [21], which is lower than a combined cycle gas turbine. However, as a type of DG, MT can be installed near the load which can greatly reduce the installation cost. Also, the emissions of MT can be controlled to an extremely low level [21].

MT is connected to a power system through a rectifier and an inverter because the AC output of an MT is usually different from the operating voltage and frequency of the distribution network, which can be seen in Figure 1-3. Since a rectifier and an inverter are important components which connect an MT to the grid and load, the control algorithms of these two electronic devices need to be studied. 


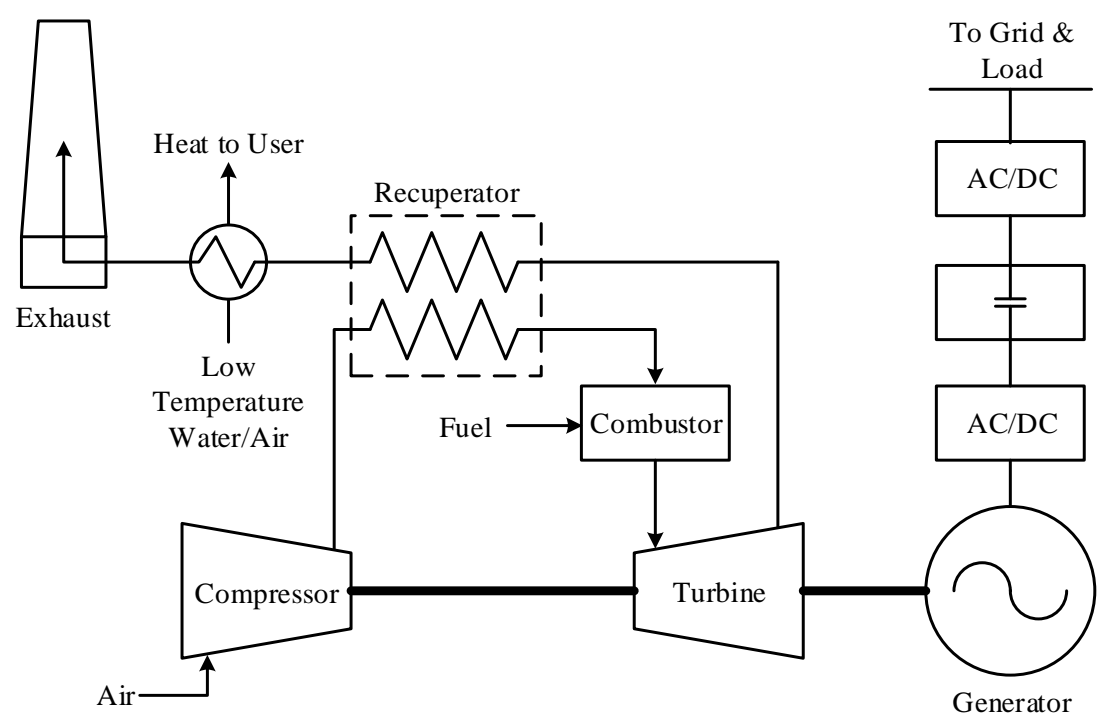

Figure 1-3: A schematic diagram of an MT connecting to grid and load.

The function of the rectifier in the configuration of Figure 1-3 is usually to maintain the DC link voltage at the desired level, the control algorithm is then to achieve the maximum power point tracking and to govern the speed of the rotating generator. As the focus of this thesis is to study the microgrid operation and its control strategy, such maximum power point tracking and speed control are not considered to study here, and the attention is given to the control algorithm of the DG inverters.

\subsection{Introduction to the Microgrid Concept}

Traditional power systems typically consist of four parts: generation, transmission, distribution, and load demand [22]. Therefore, these four parts form a gigantic network to deliver the electricity generated from online generators to end users. To solve the energy problem mentioned in Section 1.1, the integration of DG units is necessary and crucial. However, the increasing integration of DG units will bring many opportunities as well as challenges [23], [24]:

- Accommodating different types of DG units, some of which have intermittent power generation characteristics.

- Developing energy management capability to support plug-and-play functionality for different potential DG units.

- Designing demand side management (DSM) with advanced energy market functionality to encourage customer participation which can improve the system stability. 
- Maintaining system stability with the increasing number of DG inverters by designing new topologies, developing new operation and control strategies, and proposing new local control algorithms for inverter control.

Nonetheless, traditional power systems are usually developed systems with large power and energy capacities. Modifying the facilities in traditional power systems for the integration of different DG units are usually very costly.

Different definitions can be found [25], [26] for microgrid. Generally speaking, a microgrid is simply a small scaled network formed with distributed electrical power generation, storages, and loads. This aggregation of DG units and loads acts as a single controllable entity from the distribution network's perspective. Because of this, a microgrid can operate either in grid-connected condition or in islanded condition. This will require the operation and control system of the microgrid to be able to handle both operating conditions without causing a black-out.

There are many microgrid projects (finished or ongoing) that can be found worldwide. The objectives of these projects are mainly to test and develop the microgrid concept through state-of-the-art technology, and research and demonstration activities. In Singapore, under the support of the Economic Development Board (EDB) and the National Environment Agency (NEA), Renewable Energy Integration DemonstratorSingapore (REIDS), a flagship initiative led by Nanyang Technological University (NTU) to build the first and largest microgrid in the tropical region, has started in 2016 [27]. The strategy of REIDS is to promote research and development (R\&D) of microgrid technology to solve engineering, economic, environmental and societal problems, to demonstrate state-of-the-art microgrid systems, to test equipment performances, to reuse the developed solutions for Southeast Asia to promote green energy and to electrify remote communities and islands. In the project, various types of DG units are to be tested including PV panels, WTs, tidal turbines, diesel generators, BESSs, and hydrogen power-to-power system (fuel cell, electrolyzer, and storage).

United States (US) has invested a lot in microgrids. In 2016, the US Department of Energy (DOE) spent more than $\$ 10$ million in microgrid R\&D in the new $\$ 220$ million grid modernization program [28]. Finished in 2012, the Santa Rita Jail Microgrid 
project was funded by the US DOE. It aimed to demonstrate the use of the microgrid to reduce the peak demand in normal condition and sustain the operation in islanded condition. The microgrid includes WTs, rooftop PV panels, backup diesel generators, fuel cell system and BESS [29].

Microgrids can be categorized in different manners. Principally, based on the network configuration and the power types, microgrids can be divided into two large categories: AC microgrids and DC microgrids [30]. The examples of these two different microgrids are shown in Figure 1-4.

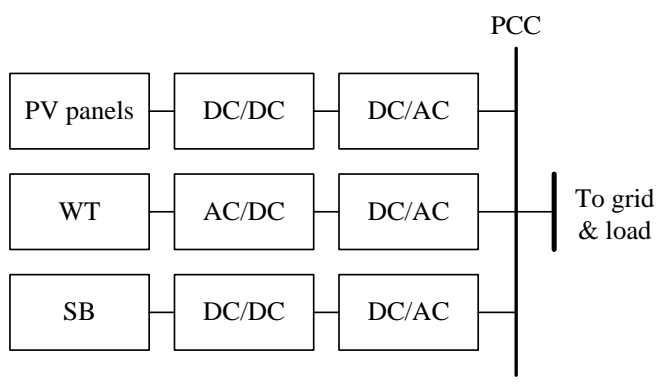

(a)

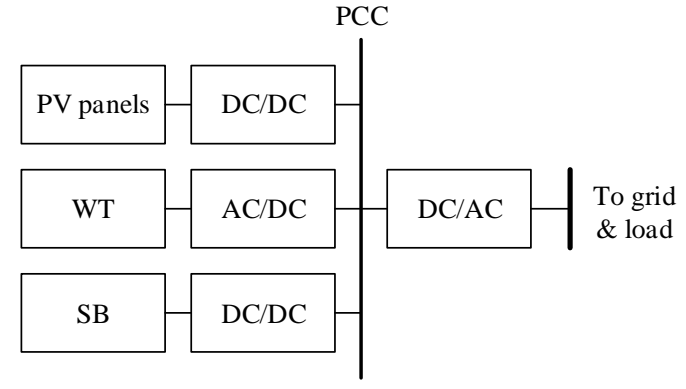

(b)

Figure 1-4: (a) An AC microgrid with PV panels, WT and SB; (b) a DC microgrid with PV panels, WT and SB.

Illustrated in Figure 1-4 (a), in an AC microgrid, different DG units are connected to the point of common coupling (PCC) through electronic devices, such as DC/DC converters and inverters to convert the power generated from the DG units to AC power [31]. Currently, AC microgrids are commonly found in various projects [32]. This is because AC power standards have been set up and used for a long time. In different countries, AC networks are widely used in transmission and distribution systems. Additionally, most of the equipment manufactured are usually according to different AC standards to be able to enter different markets. Although AC microgrids are more common and easier to build using existing industrial equipment, it is obvious that redundant inverters are used which is not economical. Aside from the economic reason, more and more loads fundamentally consume DC power. Many apparatuses, such as cell phones and computers, usually include inverters in the power modules [33] to convert the existing $\mathrm{AC}$ power to $\mathrm{DC}$ power for consumption. 
Therefore, the concept of DC microgrids has become popular in recent years. Although a DC microgrid generally has a DC grid powered with DC power, the microgrid is usually a hybrid microgrid with available DC power and AC power at the same time [34], [35]. By doing so, DC loads can be connected directly to the DC grid, while AC loads can still be connected to the PCC after the inverter.

As can be seen from the above, inverter operation and control in microgrids are important for both AC microgrids and DC microgrids. This is because the operation of a microgrid relies on DG units, while most of the DG units require DG inverters to interface with the PCC in both AC and DC microgrids.

DG inverter operation and control are globally governed by microgrid EMS as indicated in Figure 1-5. The EMS forecasts the DG units' generation and load consumption, oversees the operating status and states of the entire microgrid, and makes decisions and optimizations based on different measurements of the states according to different economic, systematic and environmental requirements. Generally, EMS will adopt different operation and control strategies to maintain normal operation and solve different problems that occur in the microgrid [26]. EMS will send control signals, such as voltage reference, frequency reference, real power reference, reactive power reference, to all the DG units in the microgrid. EMS will need to sustain the operation of the microgrid in both grid-connected and islanded conditions through the control strategies defined in it. Additionally, EMS will also activate load shedding schemes in certain situations usually when the total generation of all the DG units cannot meet the load demand, or for other optimization purposes. 


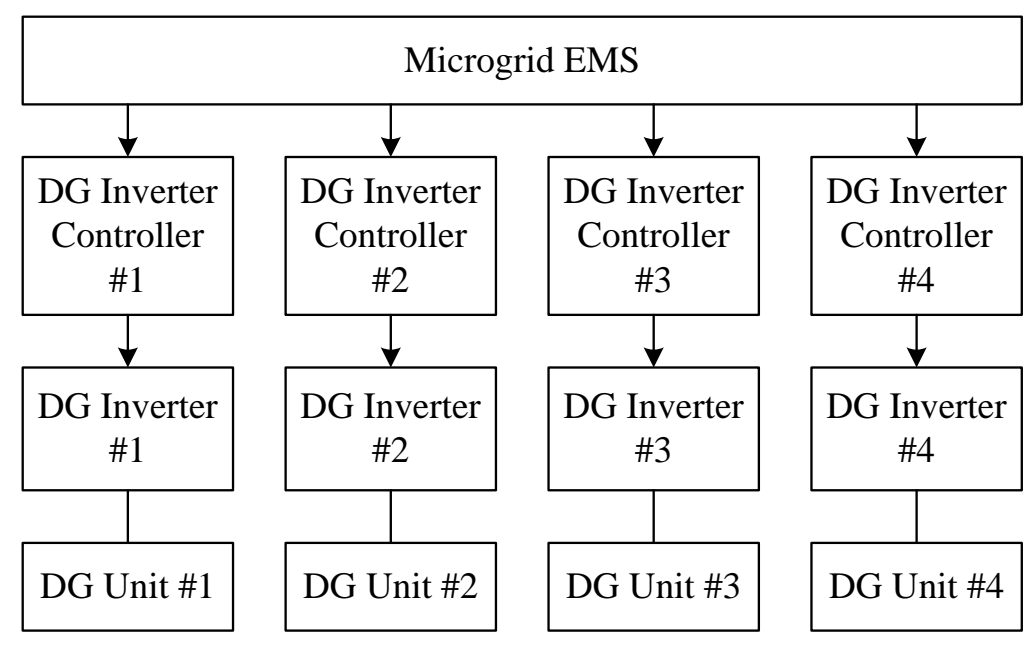

Figure 1-5: An example of the operation and control structure in a microgrid

It is observed that the operation and control strategies within EMS are closely linked to energy management, which can be inferred as a centralized approach. In a microgrid, centralized control is essential for microgrid operation and optimization.

At a lower level of the operation and control structure in a microgrid, which is shown in Figure 1-5, the DG inverter controller has the objectives to maintain the operation of each DG inverter, and regulate the voltage and power outputs of the DG unit [36]. In a centralized approach, the DG inverter controllers will receive set points directly from the microgrid EMS and regulate their outputs [37]. However, to achieve automation in a microgrid, a decentralized approach in the control structure is usually preferred [38]. Instead of relying heavily on the communication network and the continuous operation of the microgrid EMS, a decentralized approach in the DG inverter controller only uses local data which can be detected at the DG inverter side to calculate its own references.

As mentioned above, the operation and control of DG inverters in a microgrid is important to maintain the operation of the microgrid itself. However, there are many challenges in controlling DG inverters in microgrids because of the following unique characteristics:

- Modeling and control are sensitive in microgrids. In a traditional power system, the capacity of the system itself is usually very large, and the system 
can sustain huge disturbances. However, in a microgrid, small disturbances can lead to the collapse of an entire system. Therefore, the modeling and control of DG inverters need to be designed carefully to take into consideration such a high-level sensitivity.

- Parallel operation of DG inverters cannot be achieved through only decentralized control in both grid-connected and islanded conditions. In microgrids, parallel operation of DG inverters is very common since most of the DG units are connected to the network through DG inverters. For the operation of microgrids, DG inverters usually need to adopt different control algorithms in grid-connected and islanded conditions because of the lack of distribution networks in islanded condition.

- No rotating mechanism results in no mechanical inertia in islanded condition. For traditional generator, the output frequency is directly linked to the rotating mechanism. The energy stored in the mechanical inertia that comes with this rotating mechanism can help to regulate the system and sustain the operation. However, in islanded condition, DG inverters have no rotating mechanism. This makes microgrids usually exist without any mechanical inertia. The power system, especially the frequency, is greatly affected by the lack of inertia.

- The operation of DG inverters needs to be guaranteed in DC microgrids. As mentioned above, DC microgrids have attracted more and more attention recently. However, with the decrease in the number of DG inverters, the reliability of these DG inverter needs to be improved as they are the only link to the upstream distribution networks which, under normal condition, constantly deliver the necessary power to microgrids.

To overcome such challenges, the operation and control of DG inverters need to be investigated, and the following points need to be addressed:

- Adopt a dynamic model of DG inverter and use an advanced control algorithm. To model the system accurately, the dynamic model of DG inverters should be investigated and tested. More importantly, a more advanced control algorithm which corrects the control signals with feedbacks in a smaller time scale should be implemented. 
- Combine centralized and decentralized approaches in inverter control. As the control algorithms for DG inverters are usually different in grid-connected and islanded conditions, a compromised control algorithm using a decentralized approach with centralized supervision should be incorporated for DG inverters.

- Incorporate system model in DG inverters to emulate mechanical inertia for an islanded microgrid. Since the absence of mechanical inertia causes many stability problems in an islanded microgrid, system frequency model needs to be incorporated to emulate the characteristics of a traditional generator. This feature will allow DG units to work as generators, and to adopt frequency control methods in islanded condition.

- Design carefully the configuration and control strategy for DG inverters in DC microgrids. To guarantee the normal operation of the DG inverters which is the links in DC microgrids to the distribution network, multiple inverter configurations should be investigated, and related operation and control strategies need to be incorporated to sustain operation of the DG inverter links.

By considering the key points mentioned above, the operation and control of DG inverters can be ensured for the operation of the microgrid. In both grid-connected and islanded conditions, DG inverters should be controlled according to different control algorithms to sustain the operation of the microgrid [39], and this will need to be governed by EMS to manage the entire microgrid. Although islanded microgrids are regarded as an advantage of the microgrid concept in integrating different DG units, the operation and control within the microgrid need to be carefully designed to avoid the collapse of the system during unintentional islanding operations. Moreover, the parallel operation in both grid-connected and islanded conditions are important, and decentralized control algorithms are usually preferred.

\subsection{Research Motivation}

The worldwide energy problems can only be solved through the utilization of RESs. As a promising solution, microgrids are being demonstrated and investigated. Microgrids offer many advantages over traditional power systems to integrate different DG units which are geographically scattered, to sustain operation during islanded 
conditions to increase system stability and power quality, and to encourage customer participation in power system management.

In Southeast Asia, there are numerous remote areas which have limited or no electricity access. Geographically, there are many islands in this region. Because of the economic situation and government policies, many places need to be electrified [40]. However, due to economical (expensive), environmental (such as typhoons) and geographical (very far from national power networks) reasons, electrification by extending national power networks is either impossible or time-consuming. Therefore, microgrids are the best solutions for these areas. By using local RESs, different DG units can be installed and connected to microgrids. Building a transmission-less power system with the decreasing cost of the DG equipment can greatly benefit local inhabitants with low electricity cost and low environmental impact.

On the other hand, in big cities around the world, microgrids are being built to increase the system reliability [41], and to reduce fuel consumption [42]. Similar to the microgrids for electrification mentioned above, these microgrids also integrate local RESs. Different DG units are installed to harness energy from different RESs and are regulated by microgrid EMSs.

With the number of microgrids increasing worldwide, the operation and control of the microgrids needs to be addressed to limit the stability issues microgrids can cause, and use microgrids to overcome different kinds of problems brought about by different RESs. As mentioned before, fundamentally, most DG units are governed by DG inverters through DG inverter controllers. Although using advanced control algorithms can guarantee the stability of the power output of a single DG inverter, the operation and control of all DG inverters within a microgrid needs to be investigated to ensure the parallel operation of such DG inverters. Additionally, the potential of using DG inverters to overcome different problems caused by RESs and loads should be considered as well, such as generation intermittency and harmonic distortion problems. This will require the operation and control of the DG inverters to include decentralized control within the local DG inverter controllers themselves and centralized control in the microgrid EMS. A well-designed control strategy consists of a control strategy in 
the EMS, and an inverter local control algorithm in the local controller needs to be investigated and tested.

For islanded conditions, a decentralized control approach is still preferred. However, in a microgrid with all inverter-interfaced DG units, no mechanical inertia exists, resulting in the non-existence of the relationship between real power and frequency [43]. An appropriate combination of centralized and decentralized control approaches needs to be adopted during islanded condition to enable the decentralized control algorithm, e.g., frequency control algorithm.

Although most of the microgrids mentioned above are AC microgrids, DC microgrids need to be investigated as well because of obvious economic reasons and application needs. In Singapore, poultry farms are the sources for farming meat or eggs for food. These poultry farms use big ventilation fans to regulate the temperature in the farms [44], [45] for the domesticated birds. Therefore, there are opportunities to harness the excessive wind energy generated by these ventilation fans [46]. Even though the wind generated by the ventilation fans does not bring generation intermittency issues, new system configurations need to be studied to further reduce the cost of constructing a microgrid for a farm. DC microgrids have the advantage over AC microgrids as fewer inverters are required. Therefore, a DC microgrid is more suitable for the poultry farms to harness the wind power.

In a DC microgrid, the connection between the DC grid and the PCC is very important in maintaining system stability. To improve the reliability of the microgrid, multiple DG inverters should be considered in the microgrid configuration. Therefore, the parallel operation of these DG inverters needs to be investigated, and operation and control strategies need to be designed for such an implementation of DG inverters in a DC microgrid.

\subsection{Objectives of This Thesis}

Aligned with the research motivations mentioned above, this thesis focuses on the design and development of operation and control strategies for DG inverters during both grid-connected and islanded conditions in both AC and DC microgrids. 
The first objective of this thesis is to design the control algorithm for the local DG inverter controller and an operation strategy in the microgrid EMS for an AC microgrid. With this operation and control strategy, the DG inverters possess the following functions:

1) Allow parallel operation of the DG inverters, and regulate the real and reactive power outputs according to references during grid-connected condition.

2) Allow parallel operation of the DG inverters, and sustain the operation of the microgrid during islanded condition by activating backup DG units within a short response time. Adopt a droop control algorithm for load sharing among backup DG units.

3) Compensate for harmonic distortion existing in the load currents, and improve the power quality of the upstream distribution network.

As the research studies were carried out in an AC microgrid mentioned above, the operation and control of such an inverter-interfaced microgrid during islanded condition need further analysis. The second objective of this thesis is to design a combined centralized and decentralized operation and control strategy for an inverterinterfaced AC microgrid during islanded condition. This control strategy should control the DG inverters to perform the following duties:

1) Allow the DG inverters to sustain operation after the microgrid is disconnected from the upstream distribution network.

2) Adopt modified frequency control methods by incorporating a system frequency model and using a frequency based model predictive control (MPC) local control algorithm.

3) Sustain the operation of the microgrid during islanded condition by proposing a detailed control strategy in the EMS with load shedding capability.

The final objective of this thesis is to extend the inverter operation and control to DC microgrids by designing and developing a DC microgrid in the poultry farm to harness the excessive wind energy while improving the power quality and reliability of the harnessed power by a proper operating and control strategy. This inverter control strategy in the DC microgrid should possess the following features: 
1) Enable parallel operation of multiple DG inverters which link the DC grid and the PCC.

2) Reduce the effect of the switching on or off of the WTs to the microgrid operation.

3) Sustain the operation of the DC microgrid during islanded condition.

\subsection{Contributions of This Thesis}

The main contributions of this thesis can be summarized as follows:

1) This thesis investigates an AC microgrid consisting of MT, PV arrays, lithiumion SB and proton exchange membrane fuel cell (PEMFC) stacks. A centralized control approach is proposed for parallel operation of the DG inverters, which consists of two parts: inverter control algorithms in the local DG inverter controllers, and centralized control strategy in the microgrid EMS. The inverter control algorithm uses a newly developed MPC algorithm with two different control modes, i.e., current control mode (CCM) and voltage control mode (VCM). The newly developed MPC algorithm consists of steadystate subproblem and transient subproblem which have not been addressed by other researchers before. The centralized control strategy aims to manage the operation of the microgrid by using the EMS to send control signals to the DG inverter controllers. This approach allows local MPC algorithms to handle the operation of the microgrid without communication between different DG inverter controllers and loads. However, if problems occur that cannot be solved by local DG inverter controllers, the centralized control strategy will be activated by the EMS through a high-speed and reliable communication network among the EMS, DG inverter controllers and loads. During both gridconnected and islanded conditions, the EMS will be able to regulate power sharing and compensate for harmonic distortion caused by the load current. Different from other research works, the harmonic compensation function is a new development using the MPC algorithm.

2) This thesis analyzes an islanded $A C$ microgrid with inverter-interfaced $D G$ units, including PV arrays, MT and SB. A control strategy is proposed with a combined centralized and decentralized approach. The proposed control strategy aims to utilize frequency control methods under no mechanical inertia 
situation in the islanded microgrid. A frequency model is proposed which will be incorporated in the microgrid EMS. The EMS will send the voltage magnitude and frequency reference signals through a high-speed and reliable communication network among the EMS, DG inverter controllers and loads to the chosen primary DG unit, such as PV, which will regulate the voltage and frequency in the islanded microgrid. Other backup DG units, such as SB and MT, will adopt a decentralized frequency droop control by implementing a frequency droop based MPC algorithm. A modified secondary frequency control is also proposed in the EMS to restore the frequency to its nominal value. This zero-inertia circumstance is thoroughly studied and analyzed in the thesis in which not many research works are conducted. As RESs continue to increase its penetration, these analyses will be very important in improving the stability and reliability of the system.

3) This thesis also extends the research of the operation and control of DG inverters to a DC microgrid for an application to harness the excess wind energy in a poultry farm. This idea is unique compared to the conventional DC network configuration. It could bring increased energy efficiency and possible energy saving. A double-inverter configuration is proposed to connect the DC grid to the PCC to increase the system stability and reliability. A double-mode (VCM and CCM) MPC algorithm is proposed for the DG inverter controller. Parallel operation of the two inverters is guaranteed by the proposed MPC algorithm. The EMS will send reference signals to these two DG inverters through a high-speed and reliable communication network in order for the MPC algorithm to control their operation. Aside from this, the proposed control algorithm can also maintain the operation during various conditions, such as failure of one inverter, reconnection of one WG, and islanded operation. The double-inverter configuration with double-mode MPC algorithm can handle this unique application effectively, and improve the reliability of the inverter operation.

\subsection{Organization of This Thesis}

This thesis consists of six chapters arranged as follows:

\section{Chapter 1: Introduction}


This chapter describes the overall background information of DG units and the microgrid concept including the current applications, existing projects, advantages, challenges, and possible development directions. It also provides the motivation, objectives, and contributions of this thesis. The organization of the thesis is provided as well.

\section{Chapter 2: Literature Review}

This chapter records different descriptions and research works that are relevant to the operation and control of DG inverters. An overall vision relating to DG inverter operation and control is presented. Real power control and management is discussed, and related research works are reviewed in comparison to traditional power systems. Research works about reactive power control and management are also discussed, and the solutions to these operation problems are reviewed. A description of the research works relating to MPC is provided in this chapter. Operation and control strategies in local controllers and EMS from previous research works for traditional power systems are reviewed. The research works regarding the operation and control of DG inverters for both grid-connected and islanded conditions are also reviewed.

\section{Chapter 3: Centralized Control for Parallel Operation of Distributed Generation Inverters in Microgrids}

In this chapter, a centralized operation and control strategy is proposed for the DG inverters in an AC microgrid. This control strategy enables the DG units in the microgrid to sustain operation during both grid-connected and islanded conditions with the DG inverters working in parallel. The proposed operation and control strategy uses an MPC algorithm for the inverter local control, and an operation strategy incorporated in the EMS for coordination of DG units. Using DG units to compensate for harmonic distortions is also discussed in this chapter.

Chapter 4: Operation and Control of Distributed Generation Inverters in an Islanded Microgrid 
This chapter focuses on analyzing the operation of an islanded AC microgrid with inverter-interfaced DG units. A frequency model is proposed to be implemented in the control strategy incorporated in the EMS. An improved droop control based MPC algorithm is utilized for the DG inverters. Moreover, a control strategy is proposed for the DG inverters to regulate the frequency in an islanded microgrid. The proposed strategy, which includes a load shedding scheme, enables the use of a decentralized frequency droop control in a zero mechanical inertia situation.

\section{Chapter 5: Control and Operation of a DC Grid Based Wind Power Generation System in a Microgrid}

This chapter presents the design of a DC grid based wind power generation system in a poultry farm. The proposed system allows flexible operation of multiple parallelconnected wind generators by eliminating the need for voltage and frequency synchronization. An MPC algorithm that offers better transient response for different operating conditions is proposed for the local control of the DG inverters. The proposed microgrid with the operation and control strategy is capable of sustaining operation under different conditions with better system stability and reliability.

\section{Chapter 6: Conclusion and Recommendations}

This chapter discusses the overall assessment of the thesis. Areas for future research are proposed as well. 


\section{CHAPTER 2 LITERATURE REVIEW}

\subsection{Operation and Control in Microgrids}

As mentioned in the previous chapter, DG units are closely linked to RESs and ESSs. From the nature of the generation, almost all the DG units need to use power electronic converters to be connected to microgrids and distribution networks. For the DG units that generate DC power, such as PV, SB, PEMFC, etc., DC/DC converters are usually used first to regulate the input DC voltages for DG inverters and to control the voltage over the DG units themselves for different purposes such as maximum power point tracking [47], [48]. After using the DC/DC converters, DG inverters are used to convert DC power to AC power. For the DG units that generate AC power, such as WT, MT, etc., rectifiers are usually used first to convert the AC power to DC power. One of the major reasons to do so is to avoid any synchronization problem [49] when connecting these DG units to microgrids and distribution networks. After using the rectifiers, DG inverters are used to convert the DC power back to AC power. Therefore, the control of the DG inverters is critical in microgrids' operation because they basically manage all the DG units. It is of great value to conduct research on this topic.

\subsubsection{Centralized and Decentralized Approaches}

The approaches for operation and control of the microgrid can be categorized into two kinds: centralized approach and decentralized approach. However, these two approaches are usually restricted in the same hierarchical control structure. In general, the hierarchical operation and control of a microgrid, which is shown in Figure 2-1 [50], includes many elements with different applications [51], [52]:

- Local controllers which include DG unit controllers (indicated as microsource controllers (MCs)) and load controllers. These two local controllers are used to control DG converters, mostly inverters, and load demand respectively.

- Microgrid EMS (indicated as microgrid central controllers (MGCCs)) is used for control of the entire microgrid for all the DG units and loads.

- Distributed management system (DMS), which is responsible for sustaining the operation of the entire distribution network to which this microgrid belongs. It 
manages the operation of the entire medium voltage (MV) and low voltage

(LV) areas in which multiple microgrids may exist [52].

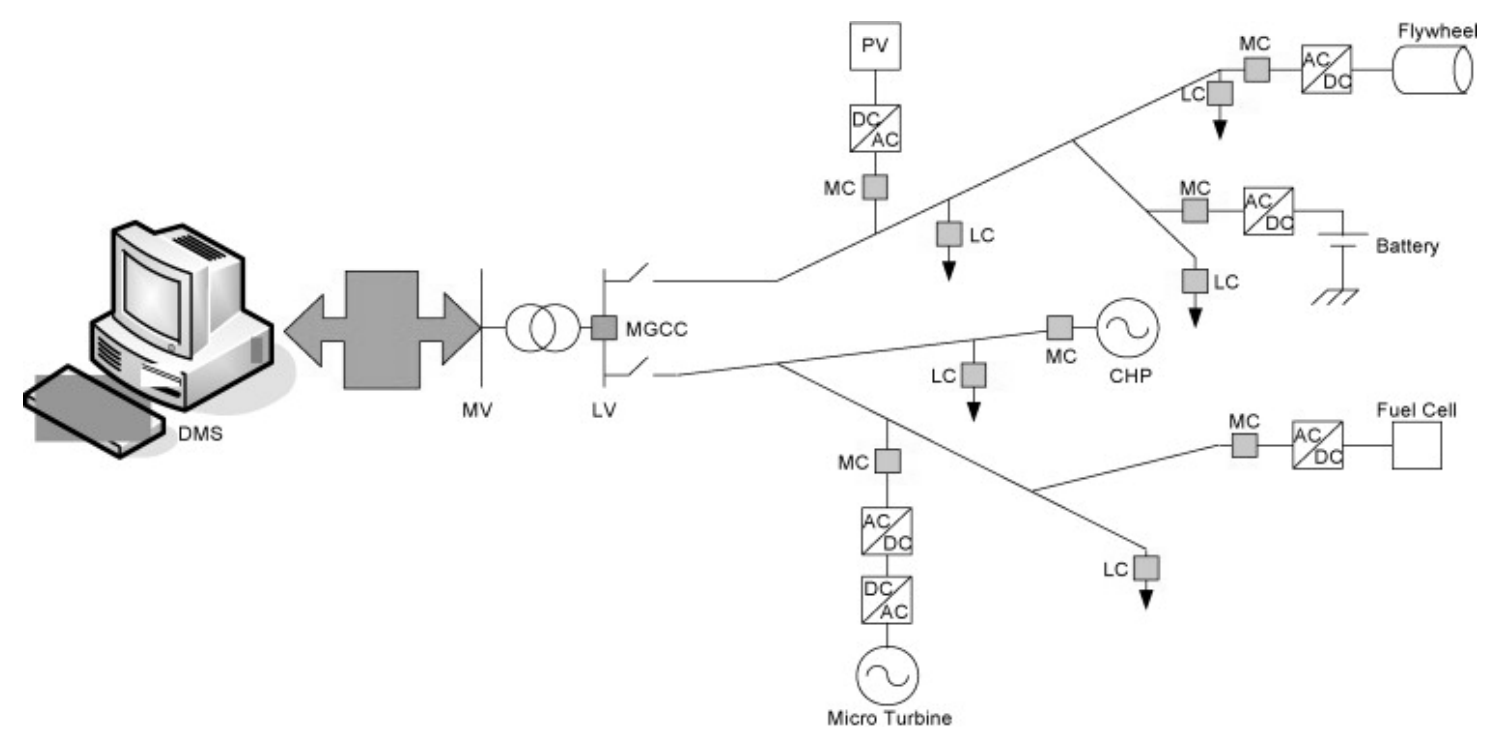

Figure 2-1: Hierarchical control of a microgrid [50].

Although centralized and decentralized control approaches have the same hierarchical control structure, the emphasis on the operation and control itself is different from one to the other.

In a centralized control approach, the microgrid EMS has the absolute authority to govern the microgrid. Optimizations and intelligent control algorithms can only be found in EMS, which sends reference signals to all the DG unit controllers and load controllers. In [53], a centralized control technique is proposed in the microgrid EMS to control the power flow in a three-phase four-wire microgrid. This proposed control technique uses a master-and-slave organization in which the EMS processes and optimizes real, reactive and unbalance power terms, and sends reference signals to all DG units. By doing so, the power quality at the PCC is enhanced, the voltage profile through the lines is improved, and the overall distribution losses are reduced. A multistage centralized control scheme for an islanded microgrid with high penetration of plug-in electric vehicles (PEVs) is proposed in [54]. The proposed control scheme utilizes a droop-based multistage optimal power flow (OPF) in the EMS to minimize load shedding during power generation shortage, to guarantee PEVs customers' satisfaction and to minimize the generation cost of the microgrid. In [55], a centralized 
control scheme using a nonlinear MPC algorithm is proposed to be applied to an islanded microgrid. The nonlinear MPC algorithm uses the SOC of the batteries, active power generation from the DG units and forecasted loads to identify upcoming active power imbalances and initiate automatic load shedding over noncritical loads. The EMS in this research performs real-time monitoring of active power generation from the DG units and load demand at each node of the microgrid.

From the above-mentioned research works, using such centralized operation and control strategies require very reliable communication networks to receive measurements from sensors and meters and send reference control signals to local controllers. In addition, the calculation burden on the microgrid EMS is heavy as all the optimizations are done within the EMS, and, sometimes, the EMS will need to handle real-time monitoring.

Therefore, to avoid the problems in the centralized approach and to maximize autonomy of the DG units and loads, decentralized operation and control strategies are proposed in many research works. As shown in Figure 2-1, in decentralized operation and control strategies, the main responsibility is given to the local controllers to integrate advanced control strategy and algorithms to sustain the operation of the microgrid without relying on the EMS. In [56], to improve the stability and security of the microgrid, a decentralized continuous coordinated control is proposed. This control scheme is based on the multi-agent system (MAS) by integrating the outer loop droop controller with the inner loop prescribed performance controller in the lower level decentralized coordination control in the MAS. To promote autonomy in a microgrid with communication constraints, a decentralized MAS based frequency control strategy is proposed and investigated in [57]. In the microgrid under study, each agent can only communicate with its adjacent agents. The control strategy needs entire system states (real power, voltage, frequency, etc.) which can be obtained by smart meters to integrate primary and secondary frequency control and multi-stage load shedding to restore the system frequency back to its nominal value. A DC microgrid consisting of PV, ESS has been investigated in [58]. A decentralized control strategy, which achieves power sharing considering the effects of line resistances, is proposed. The control strategy integrates several different working modes in the DC microgrid to 
ensure the stability of the system. The SOC of the batteries is also considered to provide reliable and prolonged operation.

However, as the operation and control of microgrids continue to develop, only using centralized control approach or decentralized control approach does not seem to be the robust solution for microgrids under different situations. Therefore, researchers are exploring possibilities to incorporate these two different approaches for the operation and control of microgrids. Similar to that shown in Figure 1-5, authors in [59] propose a design of a microgrid EMS for optimal operation taking into consideration the distribution network and associated constraints. In this design, the microgrid central controller, which is mostly referred to as the microgrid EMS, works together with the local controllers to compute an optimal operating schedule. Even though this method proposed in [59] combines centralized and decentralized approach, the communication burden in its communication network is intense, and this could cause potential reliability problems.

\subsubsection{Operation and Control of Grid-Connected and Islanded Microgrids}

Aside from the centralized and decentralized strategies, one of other biggest characteristics of the operation and control of a microgrid is that there are two operating conditions, grid-connected and islanded conditions. As one of the advantages of the microgrid concept, the ability to operate in these two different operating conditions can definitely increase the reliability of the system. However, the operation and control strategies towards these two operating conditions vary from one to the other.

In the grid-connected condition of a microgrid, the upstream distribution network will supply a certain percentage of total power consumed by the loads, while the DG units will supply the remaining power. The upstream distribution network is usually regarded as an infinite bus which can absorb power fluctuations and solve power shortage problems. Therefore, the operation and control strategy in grid-connected microgrids are usually very flexible. However, power sharing problems and additional functions, such as compensating for unbalanced loads, which can be provided by grid- 
connected microgrids are still important. In [60], a distributed control scheme is proposed for grid-connected AC microgrids. In this control strategy, each DG unit is equipped with a local observer to communicate with each other for reference control signals. A local tracking controller is also proposed for each DG unit to track the computed reference control signal. The operational performance of droop control strategy in a grid-connected microgrid is analyzed in [61]. An enhanced power flow control strategy is proposed without extra sensors. The proposed control strategy consists of the feedforward of the grid voltage magnitude and the grid frequency to reduce the impact from the fluctuation of the upstream distribution network. It also includes a voltage magnitude control loop to improve control accuracy of reactive power flow. In addition, the system stability is enhanced by this enhanced power flow control strategy. Aside from power sharing and power flow control problems, a current control strategy for the DG inverters in grid-connected microgrids to compensate for unbalanced or nonlinear loads is proposed in [62]. This control strategy can effectively compensate for the harmonic current content of the upstream distribution grid and microgrid by using no compensation devices such as active, passive and LCL filters.

On the contrary, there are many challenges in the operation and control of islanded microgrids. Without the upstream distribution network, an islanded microgrid needs to use proper operation and control strategy to avoid the collapse of the microgrid. A novel operation and control strategy for an islanded microgrid to regulate the frequency and voltage using multiple ESSs is proposed in [63]. This control strategy combines the $v-f$ droop control and the $P-Q$ droop control. Without involving the line parameters uncertainty, this control strategy can distribute real and reactive power precisely, and improve system stability. In [64], a control strategy to achieve harmonic current sharing in an islanded microgrid is proposed. This control strategy directly controls the inherent impedance of the DG inverters without using any communication infrastructure. In addition, a droop technique to distribute the harmonics among the different DG units in the microgrid is provided.

It can be seen from the above description that researchers are dedicated to proposing and improving different operation and control strategies to solve different problems which may occur in both grid-connected and islanded conditions. Furthermore, linking the two operating conditions to the centralized and decentralized approaches will 
provide a clearer global vision on the operation of the microgrid. Previous research works usually separate completely these two operating conditions. Operation and control strategies usually cannot apply to both operating conditions as the system characteristics are different.

\subsection{Real Power Control and Management in Microgrids}

Real power control and management is one of the most important aspects of operation and control of microgrids because real power (or active power) is the power that does the actual work, e.g., creating heat, lifting loads, etc.

\subsubsection{Local Control in DG Inverters}

In traditional power systems, generators are the major power sources to generate electricity using fossil fuels such as coal, natural gas, etc. Each generator has its own rotating mechanism which is powered by a prime mover. Therefore, it is obvious that generated electrical power is directly related to this rotating mechanism which is the link between mechanical energy, produced by fossil fuels, and electrical energy. Also, the rotational speed is directly linked to real power output of the generator which can be indicated by the frequency of the power system. Any real power imbalance between generation and load demand in the power system will cause the rotational speed of the online generators to change, thus resulting in system frequency variation. Because of this characteristic, frequency control methods are widely used in traditional power systems for real power control for generators.

Among various control methods, load frequency control (LFC) is one of the most commonly used frequency control method in traditional power system. The main objective of LFC is to maintain the system frequency within an acceptable range by controlling the real power outputs of the generators which are connected to the grid and the tie-lines' real power exchanges [65]. In LFC algorithm, the system frequency could be formulated as a function of real power relationship between power generation and load. 
Figure 2-2 shows the block diagram of a dynamic LFC for an interconnected power system. In this figure, $\Delta P_{\text {ref }}$ represents the change in the setting of real power output by LFC controller, $\Delta P_{g}$ represents the change in the setting of real power output of the generator, $\Delta P_{V}$ represents the change in the real power output from the valve side, $\Delta P_{T}$ represents the change in the real power output from the turbine, $\Delta P_{L}$ represents the change in the real power of the load, $\Delta f$ represents the change in the system frequency, and $R$ is the droop characteristic for the frequency/real power relationship. The transfer functions in the figure, namely $G_{H}, G_{T}$, and $G_{P}$, are the transfer functions for the hydraulic process, turbine rotating process and real power/frequency relationship, respectively.

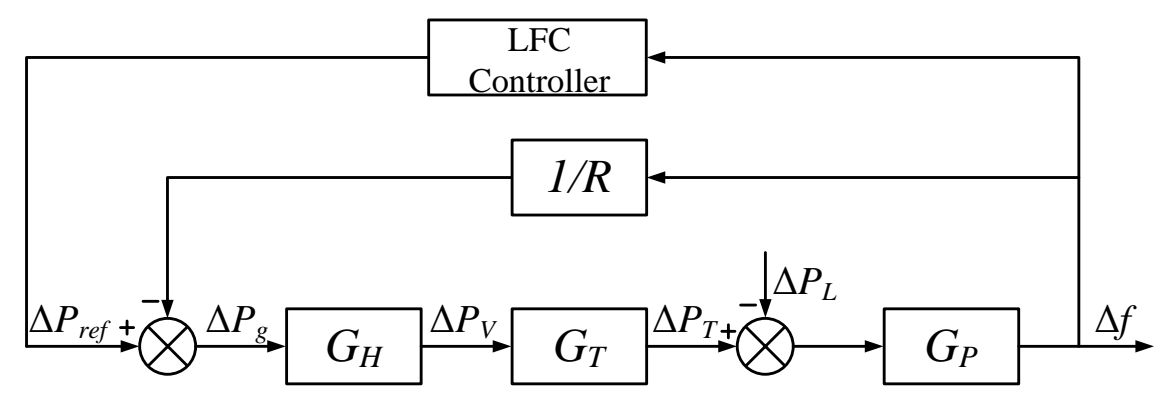

Figure 2-2: General block diagram of the dynamic LFC.

From Figure 2-2, aside from the LFC controller in the feedback control loop, there is a frequency droop control automatically integrated for the generators to adjust their real power output. Similarly, for a grid-connected microgrid, the DG inverters can use artificial decentralized frequency droop control to achieve load sharing between the different DG units. Figure 2-3 shows the real power versus frequency $(P-f)$ droop characteristics, which are usually incorporated in the frequency droop control of a DG unit. The relationship is given by:

$$
f=f_{0}-K_{P}\left(P-P_{0}\right)
$$

where $f$ is the operating frequency of the DG unit, $f_{0}$ is the nominal frequency, $K_{P}$ is the droop slope of the DG unit which is usually determined based on historical data of the steady-state operation [66], [67], $P$ is the actual real power output of the DG unit, 
and $P_{0}$ is the nominal reference real power output based on the power rating of the DG unit. This is to maintain the system frequency within an allowable range in the power system that the microgrid belongs to. From Figure 2-3, it is observed that when there is a problem in the power system which causes the system frequency to drop, e.g., a sudden increase in the load, the DG unit will self-regulate to boost up its real power output to $P_{1}$ to help maintain the operation of the system. However, the power system will stay in under-frequency condition as $f_{1}<f_{0}$. Illustrated in Figure 2-3, the operating point of the system has shifted from Point $a$ to Point $b$ because of the frequency drop. The primary droop control cannot restore the system frequency back to its nominal value of $f_{0}$. Therefore, a secondary frequency control should be utilized in the system to change the system operation state from Line 1 to Line 2, thus moving the operation point to Point $\mathrm{c}$ and restoring the system frequency to $f_{0}$.

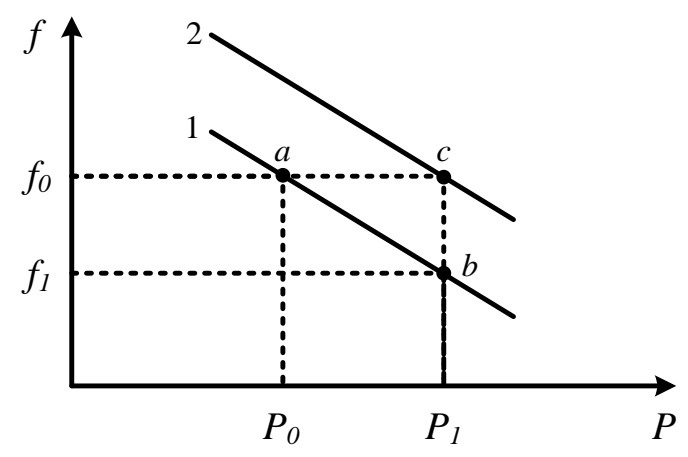

Figure 2-3: Real power versus frequency $(P-f)$ droop characteristics.

However, in an islanded microgrid, because of the loss of the rotating mechanism of the online generators and the fact that the DG units are usually connected to the microgrid through DG inverters, the natural relationship between the system frequency and the real power does not exist anymore. Without mechanical inertia, an islanded microgrid cannot adopt the mentioned decentralized frequency droop control. Autonomous control strategies using $P-f$ droop characteristics are proposed in [68]. These droop characteristics are modified to be adjusted locally in the local DG inverter controllers in real-time, based on the available PV power, load demand and the SOC of the battery. The control strategies are using multi-loop controllers without the need of an EMS. Yet, the calculation burden for the local controllers become quite heavy to include such control strategies. A robust control designed for both grid-connected and 
islanded conditions can be found in [69]. This control scheme includes two levels of control: one centralized controller which computes and sends real and reactive power reference signals to all the DG units, and the local DG inverter controllers which use droop control. The control scheme can sustain the operation of the microgrid during islanding transients as well. In [70], a coordinated architecture of an islanded AC microgrid with a smooth switching droop control strategy is proposed. This proposed control strategy uses a decentralized approach for the power management by executing frequency bus-signaling. It ensures flexible power control of each DG unit with seamless mode changes between VCM and CCM in the DG inverter controllers.

\subsubsection{Real Power Management in Microgrid EMS}

As observed from LFC in traditional power systems, aside from a local frequency droop characteristic integrated in the control of a generator, an LFC controller is also needed which considers the configuration of the power systems such as tie-lines. Similarly, in microgrid real power management, control strategies are also proposed in microgrid EMS to manage the real power generation and consumption.

In [71], a real power management strategy, based on an ultra-short-term power prediction and a feed-forward control, is proposed in the microgrid EMS. In a microgrid consisting of WT, BESS, PV, diesel generator and factory load; the control strategy is proposed, as shown in Figure 2-4. It can be seen in this figure that the EMS uses real-time load detection and microgrid network states, conducts an ultra-shorttime prediction, controls all the DG units in a feed-forward fashion by sending the reference control signal to the local controllers.

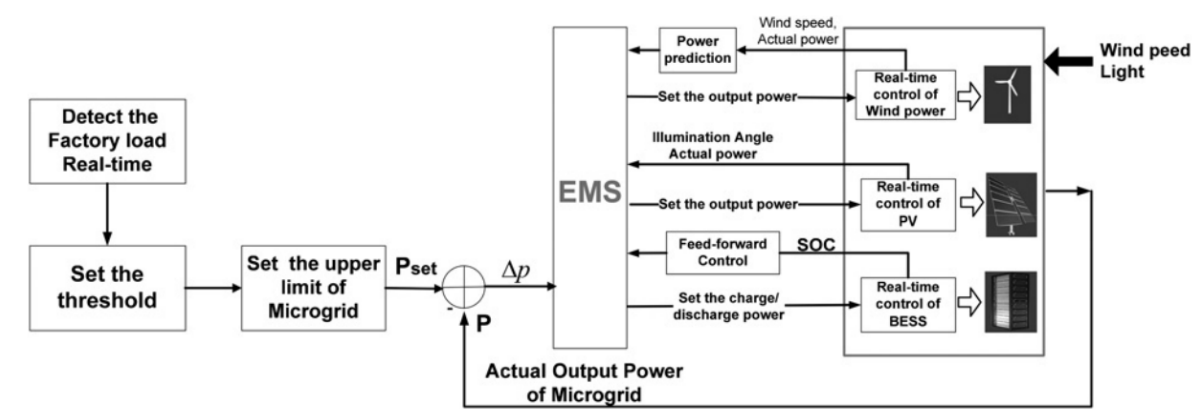

Figure 2-4: Control strategy proposed in the EMS [71]. 
In the strategy proposed as Figure 2-4, the local controllers of the DG inverters do not perform additional functions other than maintaining their real power outputs at the reference values provided by the microgrid EMS. Similarly, in [72], a real power control strategy is proposed for a laboratory scale microgrid in the microgrid EMS. It is a DC microgrid consisting of WT, PV, SB, and AC load. The proposed control strategy in the EMS coordinates the control of the DC/DC converters and DG inverters. As illustrated in Figure 2-5, the proposed control strategy regulates the real power generated by the DG units by using system states to calculate real power generation and consumption, and SOC of the SB. Corresponding actions, such as charging the SB, shedding load, etc., are proposed to sustain the operation of the microgrid under study.

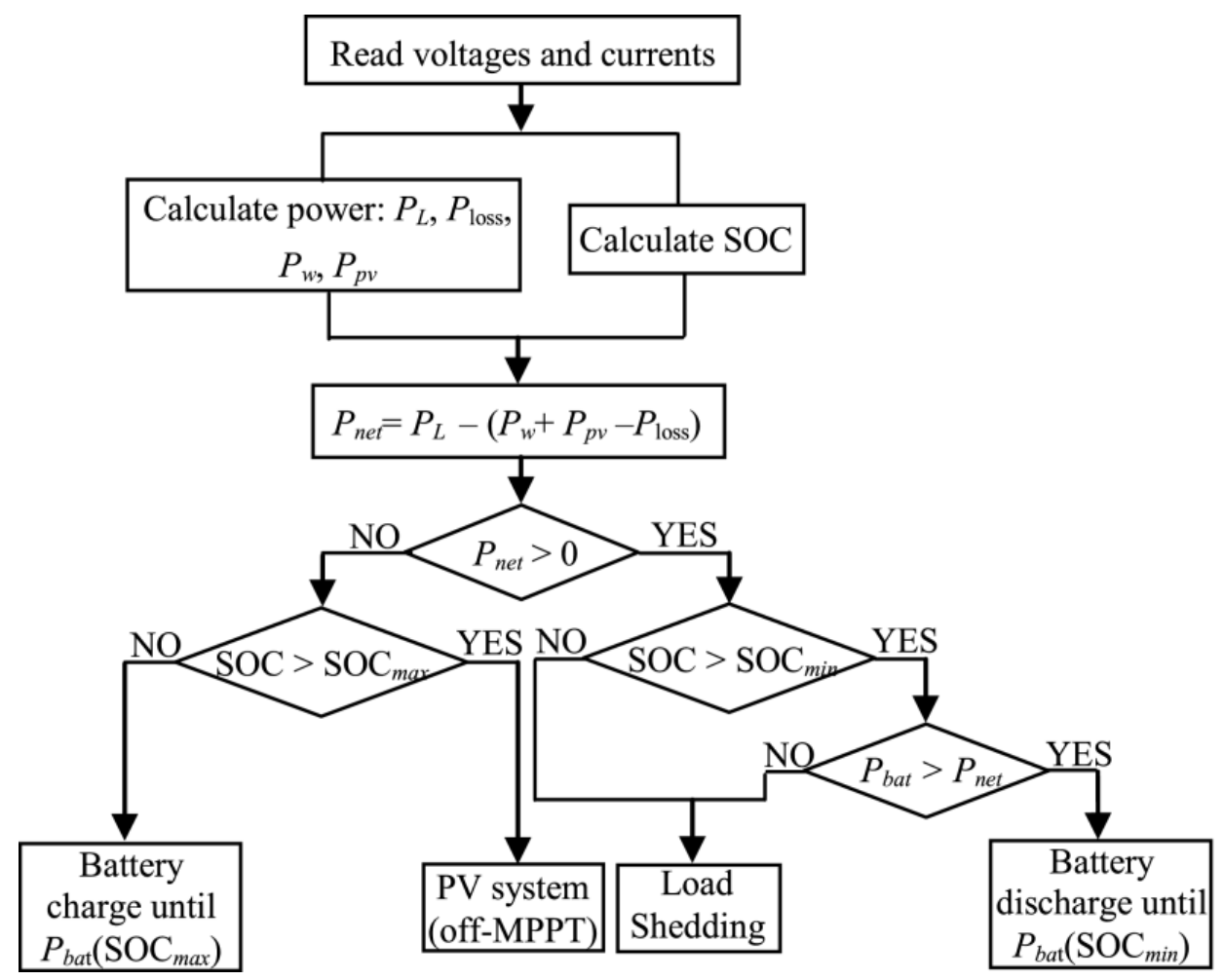

Figure 2-5: Control strategy proposed in the microgrid EMS [72].

Such control strategy diagrams will be further detailed in a later section. However, using such control strategies in the EMS to control and manage real power by sending different reference signals is very common. 


\subsection{Reactive Power Compensation in Microgrids}

In traditional power systems, reactive power is linked with the voltage magnitude of the electrical bus. Even though reactive power cannot do actual work as real power, it is necessary that for a specific bus, the reactive power is balanced. Also, the above statements are also applicable for microgrids. As microgrids are usually small scaled distribution networks, the line impedances are quite small, sometimes they are neglected in research works [73], [74]. Therefore, the reactive power balance in a microgrid is usually considered all together, which means that the injected reactive power (capacitive) should be equal to the absorbed reactive power (inductive) in the microgrid.

There are several different methods to compensate for reactive power in traditional power systems:

- Shunt capacitor: After first used in 1914 [75], the shunt capacitor injects reactive power into the power system to compensate for the lagging current drawn by the load. However, a fixed capacitor could cause either overcompensation or under-compensation. To solve this problem, a switched capacitor [76] is used to achieve variable reactive power compensation. Yet, this method is based on mechanical switches and relays, and it is sluggish and unreliable. The inrush current it generates can be very high, and the maintenance cost can be quite expensive.

- Synchronous condenser: A synchronous condenser is simply a synchronous machine which is synchronized to a power system which is adjusted either to generate or absorb reactive power as required. It can provide continuous reactive power support with a high temporary overload capacity. However, because of the use of a synchronous generator, it requires a significant amount of capital cost as well as starting and protective equipment. In addition, the losses in a synchronous condenser can be very high.

- Static VAR compensator (SVC): An SVC is a power electronic device which can compensate for a wide reactive power range with a faster response time [77]. It is usually labeled under two different categories: thyristor controlled reactor and thyristor switched capacitor. 
- Static synchronous compensator (STATCOM): A STATCOM is an inverter based device which converts a DC input to an AC output to compensate for the active and reactive needs of the power system. The STATCOM has better performance than the SVC in terms of compensation characteristics and response time.

As it can be seen from the above-mentioned methods in traditional power systems, STATCOM is very close to the DG unit configuration with DG inverter to control the output. Therefore, most research works use DG units to compensate for the reactive power by controlling the DG inverters to share the absorbed reactive power by the load or the upstream distribution network. In [78], a stochastic reactive power compensation control strategy is developed to control the DG inverters to compensate for reactive power for the microgrid depending on the power generation and consumption data. This centralized control approach can handle the intermittency of RESs in the microgrid. A reactive power compensation technique, based on voltage sag and power flow analysis, is proposed in [79]. This application is designed for the microgrid which feeds single-phase loads with feeders located far from each other covering small communities. It uses a droop based centralized control algorithm to generate reactive current references for the different DG units. It also relies on a communication network for which the data transfer requirements are provided in this research.

However, reactive power compensation methods in traditional power system suggest that the reactive power and the harmonics are usually locally compensated to keep the online generators working at unity power factor. In a microgrid, without any reactive power compensation equipment, the DG units should compensate for the reactive power consumption of the load. For full compensation, the summarized ratings of the DG units in a microgrid should be capable of compensating for all the reactive power absorbed by the load. To share the reactive power generation among the DG units, voltage droop control is usually adopted. Figure 2-6 shows the relationship between reactive power versus voltage $(Q-v)$ droop characteristics, which are usually incorporated in the voltage droop control of a DG unit. The relationship is given by: 


$$
v=v_{0}-K_{Q}\left(Q-Q_{0}\right)
$$

where $v$ is the actual voltage magnitude at the output terminal of the DG unit, $v_{0}$ is the nominal voltage magnitude, $K_{Q}$ is the droop slope of the DG unit which is usually determined based on historical data of the steady-state operation [66], [67], $Q_{0}$ is the nominal reference reactive power output based on the power rating of the DG unit, and $Q$ is the actual reactive power output of the DG unit.

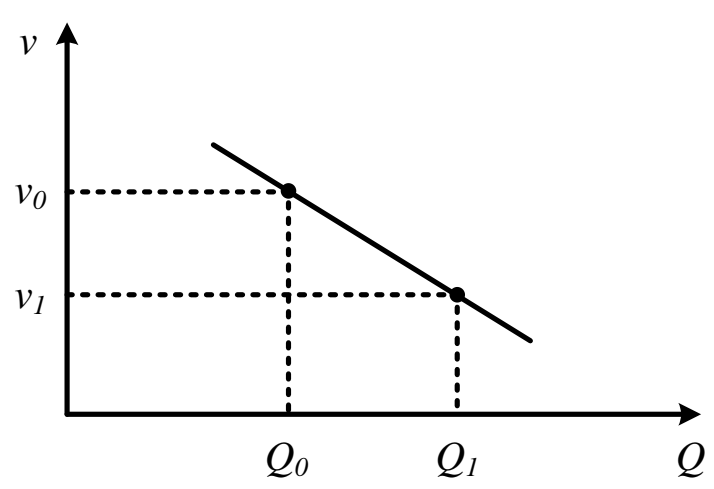

Figure 2-6: Reactive power versus voltage $(Q-v)$ droop characteristics.

Similar to the real power operation and control, DG units in microgrid are most likely to work in CCM during grid-connected condition while the online generators are maintaining the voltage of the upstream distribution network which can represent an infinite bus. The voltage magnitude at the PCC will be affected along with the change in reactive power supplied by the upstream network. Therefore, the DG inverters can adjust their reactive power output accordingly. However, during islanded condition, losing the connection to the upstream distribution network will make the DG units work in VCM where the DG inverters control the voltage magnitude and frequency of their outputs. If line impedances are not neglected in the islanded microgrid, they are mainly resistive compared to traditional power systems in which the transmission lines are mainly inductive. These will change the relationship between reactive power and voltage. To solve this reactive power compensation problem in islanded condition, a power sharing controller with droop characteristics that consider resistive line impedances are proposed in [80]. The controller consists of three control loops including $P / Q$ sharing control loop, a resistive virtual impedance loop, and an inner 
voltage control loop. In [81], the authors propose a stationary frame control method for voltage unbalance compensation in an islanded microgrid. The proposed control strategy includes real and reactive power droop controllers with a virtual impedance loop, voltage and current controllers and an unbalanced compensator.

\subsection{DG Inverter Control Methods}

For the operation and control of microgrids, the hierarchical control structure in Figure 2-1 shows that in order to manage and control real and reactive power, as mentioned in the previous section, control algorithms in the DG inverter controllers are the fundamental elements. For the DG inverter control, based on the different frames to formulate the problem, it can be categorized into the following groups: synchronous reference frame $(d q 0)$ control, stationary reference frame $(\alpha \beta \gamma)$ control, and natural frame $(a b c)$ control.

- Synchronous reference frame $(d q 0)$ : This frame is a transformation of the three-phase AC system into two directional DC quantities. When the rotating frame is aligned with phase $a$, the transformation can be illustrated as follows:

$$
\left[\begin{array}{l}
v_{d}(t) \\
v_{q}(t) \\
v_{0}(t)
\end{array}\right]=\frac{2}{3}\left[\begin{array}{ccc}
\cos (\omega t) & \cos \left(\omega t-\frac{2 \pi}{3}\right) & \cos \left(\omega t+\frac{2 \pi}{3}\right) \\
-\sin (\omega t) & -\sin \left(\omega t-\frac{2 \pi}{3}\right) & -\sin \left(\omega t+\frac{2 \pi}{3}\right) \\
\frac{1}{2} & \frac{1}{2} & \frac{1}{2}
\end{array}\right]\left[\begin{array}{l}
v_{a}(t) \\
v_{b}(t) \\
v_{c}(t)
\end{array}\right]
$$

where $v_{d}, v_{q}$ and $v_{0}$ are the voltages transformed in the $d q 0$ frame as dcomponent, q-component and 0-component, respectively, and $v_{a}, v_{b}$ and $v_{c}$ are the voltages in the natural $a b c$ frame as phase $a$, phase $b$ and phase $c$, respectively. For a balanced system, $v_{0}$ is zero. The transformation is to reduce the complexity of the control and filtering for a complicated three-phase power system. A typical control algorithm in the $d q 0$ frame is the classic PI control, whose transfer function can be generally represented as follows [82]: 


$$
G_{P I}^{d q 0}(s)=\left[\begin{array}{cc}
K_{p}+\frac{K_{i}}{s} & 0 \\
0 & K_{p}+\frac{K_{i}}{s}
\end{array}\right]
$$

where $K_{p}$ is the proportional gain of the PI controller, and $K_{i}$ is the integral gain of the PI controller. A general configuration using PI control in the $d q 0$ frame for the DG inverter is shown in Figure 2-7.

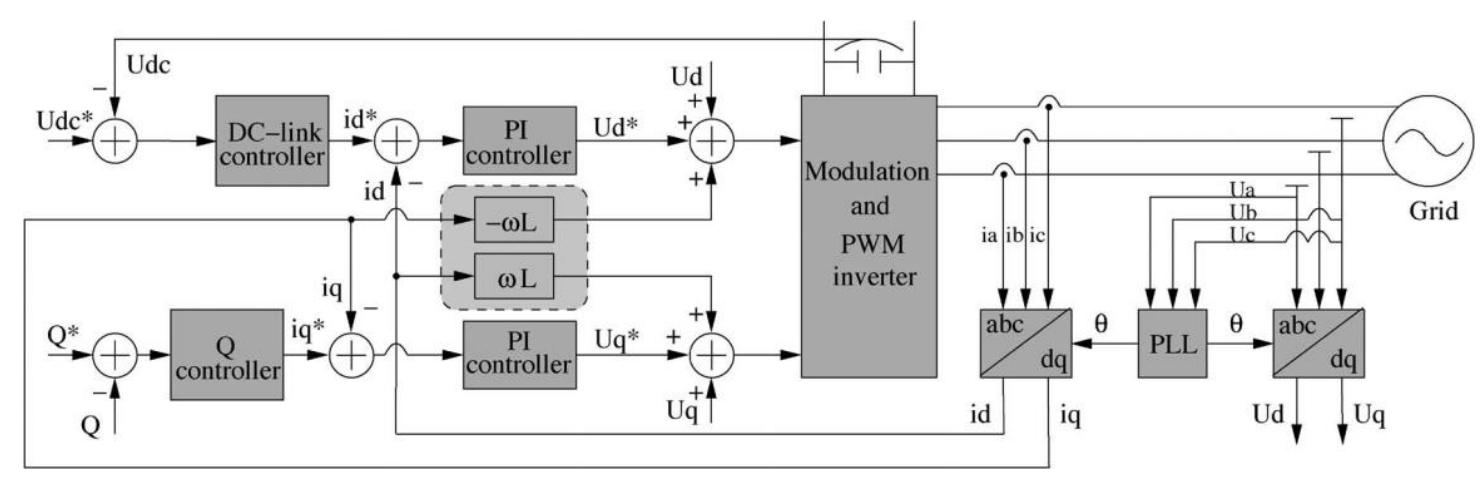

Figure 2-7: General PI control configuration in the $d q 0$ frame [83].

- Stationary reference frame $(\boldsymbol{\alpha} \boldsymbol{\beta} \gamma)$ : This frame is through a transformation known as Clarke transformation. It is similar to the transformation of the $d q 0$ frame; the general transformation can be described as follows [83]:

$$
\left[\begin{array}{l}
i_{\alpha}(t) \\
i_{\beta}(t) \\
i_{\gamma}(t)
\end{array}\right]=\frac{2}{3}\left[\begin{array}{ccc}
1 & -\frac{1}{2} & -\frac{1}{2} \\
0 & \frac{\sqrt{3}}{2} & -\frac{\sqrt{3}}{2} \\
\frac{1}{2} & \frac{1}{2} & \frac{1}{2}
\end{array}\right]\left[\begin{array}{l}
i_{a}(t) \\
i_{b}(t) \\
i_{c}(t)
\end{array}\right]
$$

where $i_{\alpha}, i_{\beta}$ and $i_{\gamma}$ are the currents of the $\alpha$-axis component, $\beta$-axis component, and $\gamma$-axis component, respectively in $\alpha \beta \gamma$ frame, and $i_{a}, i_{b}$ and $i_{c}$ are currents in natural reference frame as phase $a$, phase $b$ and phase $c$, respectively. In a symmetrical power system, $i_{\gamma}$ is zero. PR control method is usually implemented in the $\alpha \beta \gamma$ frame. The general transfer function of a PR control can be expressed as follows [84]: 


$$
G_{P R}^{\alpha \beta \gamma}(s)=\left[\begin{array}{cc}
K_{p}+\frac{K_{i} s}{s^{2}+\omega^{2}} & 0 \\
0 & K_{p}+\frac{K_{i} s}{s^{2}+\omega^{2}}
\end{array}\right]
$$

where $\omega$ is the resonance $\mathrm{AC}$ frequency, $K_{p}$ is the proportional gain, and $K_{i}$ is the integral gain. Also, a typical control configuration using the PR control method for the DG inverters is shown in Figure 2-8. It is observed that it is quite similar to the configuration of the PI control.

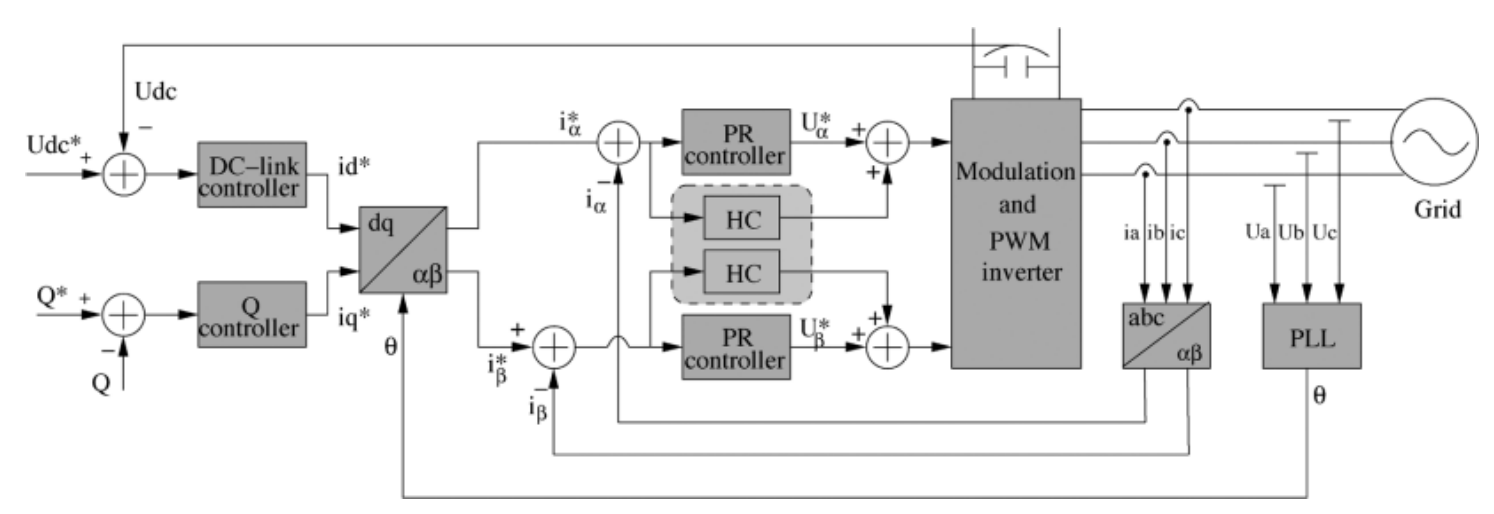

Figure 2-8: General PR control configuration in the $\alpha \beta \gamma$ frame [83].

- Natural frame $(\boldsymbol{a b c})$ : This frame is basically used to naturally distinguish among the three phases. Even though the three-phase system is a dynamic system in which currents and voltages are all sinusoidal waveforms in time domain, the $a b c$ frame is a structure where nonlinear controllers such as hysteresis or deadbeat controls are preferred due to their high dynamics [83]. In this frame, PI controller is possible which is detailed in [85]. PR control method is also straightforward, extending the existing transfer function. Other than these two, hysteresis control can be implemented by obtaining the fixed switching frequency [86], [87]. In addition, deadbeat control can be implemented in the $a b c$ frame. But deadbeat control will introduce one sample time delay as it regulates the current to reach its reference at the end of the next switching period [88]. Usually by introducing an observer, this delay can be compensated. 


\subsection{MPC Implementation in Microgrid}

It can be seen in the previous section, the commonly used DG inverter local control algorithms are PI, PR, hysteresis, and deadbeat controls. Even though these dynamic controllers are verified in industrial implementations, and research works which improve these control algorithms are pushing the controls to their limits [89], [67], it will be very difficult to add advanced features and complicated operational strategies within these control algorithms to the local DG inverter controllers. In addition, as the development of information technology has improved greatly during the last decades, advanced control algorithms are becoming more and more popular in academic research works as well as industrial implementations.

As one of the most promising advanced control algorithms, the MPC algorithm has been implemented in various areas including electrical power systems. MPC generally refers to any control algorithm which uses mathematical equations to model a specific system and solve an optimization problem within this specific system. The models that are used in MPC are intended to represent the behaviors of real and complex dynamic systems. A general mathematical discrete model of a system can be expressed in a state-space form as follows:

$$
\left\{\begin{array}{c}
\mathbf{x}(k+1)=\mathbf{A x}(k)+\mathbf{B u}(k) \\
\mathbf{y}(k)=\mathbf{C} \mathbf{x}(k)+\mathbf{D u}(k)
\end{array}\right.
$$

where $\mathbf{x}$ is the system state variable matrix, $\mathbf{y}$ is the output matrix, $\mathbf{u}$ is the control signal matrix, and $\mathbf{A}, \mathbf{B}, \mathbf{C}$ and $\mathbf{D}$ are coefficient matrices to model the system dynamics. MPC uses the model to predict a certain amount of time defined by the algorithm. A cost function $J$ is then defined to formulate a control problem into an optimization problem which needs to be solved by minimizing the cost function $J$. Therefore, $J$ is usually formulated as the summation of the deviations between different system states and their reference values. By minimizing $J$, reference control signals can then be calculated using various kinds of methods to solve the optimization problem. For the system in (2.7), a possible general cost function can be defined as follows: 


$$
J=\sum_{i=1}^{N_{p}} w_{y_{i}}\left(r_{i}-y_{i}\right)^{2}+\sum_{i=1}^{N_{p}} w_{u_{i}}\left(u_{i-1}-u_{i}\right)^{2}
$$

where $y_{i}$ is the $i$ th state variable in $\mathbf{y}, r_{i}$ is the reference value for the $i$ th state variable, $u_{i}$ is the $i$ th control signal ( $u_{0}$ is usually equal to zero) in $\mathbf{u}, w_{y_{i}}$ is the weight coefficient for $y_{i}, w_{u_{i}}$ is the weight coefficient for $u_{i}$, and $N_{p}$ is the prediction horizon. From (2.8), MPC can predict and consider multiple time steps into the future, defined in $N_{p}$. However, in most cases, the control signals generated from this optimization, which means $u_{i}\left(i=1,2, \ldots, N_{p}\right)$ are not all executed. An $N_{c}$ which satisfies $N_{c} \leq N_{p}$ is usually defined such that in the system where $N_{p}$ control signals are generated from the optimization, only $N_{c}$ control signals will be executed which refers to $u_{i}\left(i=1,2, \ldots, N_{c}\right)$. This methodology is commonly known as the rolling horizon control or receding horizon control. In addition, because MPC algorithm transforms the operation problem of a system to an optimization problem, it is very easy to incorporate additional constraints, as many optimization algorithms naturally support constraints such as mixed integer linear programming.

Because of the use of the optimization problem formulation which involves heavy calculation, MPC is mostly introduced in microgrid operation management that does not need real-time monitoring of the system. An MPC based home energy management system for a residential microgrid is proposed in [90] by using various information, such as load demand, the electricity tariff, and the real power generation of the DG units. This operation strategy functions at a sampling time of 1 hour, and it can successfully reduce the overall cost of the microgrid. Similarly, as in [91], an advanced control strategy based on a two-layer MPC for optimal microgrid operation is proposed. This MPC control algorithm uses mixed integer nonlinear programming to formulate the problem in the nonlinear system model presented in this paper. The first layer of the MPC algorithm determines the optimal power flow while the second layer adjusts the ON/OFF status of the diesel generator. Another microgrid operation management strategy is proposed in [92] using MPC to efficiently optimize the microgrid operation according to the operating cost while satisfying a time-varying request and system constraints. This MPC algorithm is formulated using mixed integer linear programming. 
From the abovementioned, it is clear that many researchers are using MPC algorithm for the operation management of the microgrid. However, as information technology continues to develop, the computational capability of a local controller is sufficient to handle simple optimization problems. Therefore, research works focusing on implementing MPC algorithm in local controller for DG inverter in microgrid become more and more popular.

Among different MPC algorithms for DG inverters, the finite control set MPC (FCSMPC) [93] is one of the most representative algorithms, which is already implemented in hardware setups. Regarding the control of the DG inverters, the fundamental control signals are the switching signals for the switches in a three-phase DG inverter. For traditional local control algorithms, such as PI control, PR control, etc., a PWM generator is necessary to generate the direct switching signals for all the switches. Therefore, to avoid using a PWM generator and generate the switching signals directly from the local controller which uses MPC algorithm, the FCS-MPC algorithm, which simplifies the network configuration by analyzing the voltage space vectors, is well acknowledged.

The FCS-MPC basically generates the control signals for all the switches by using the possible finite control actions which refer to the switching states. A general configuration of a three-phase DG inverter is represented as in Figure 2-9. From Figure 2-9, there are six switches in total in the DG inverter. The six switches are divided into three pairs controlling the output of the three phases. In each pair, the switches cannot be opened or closed at the same time. Therefore, there are eight control sets in total which can be given to these switches. 


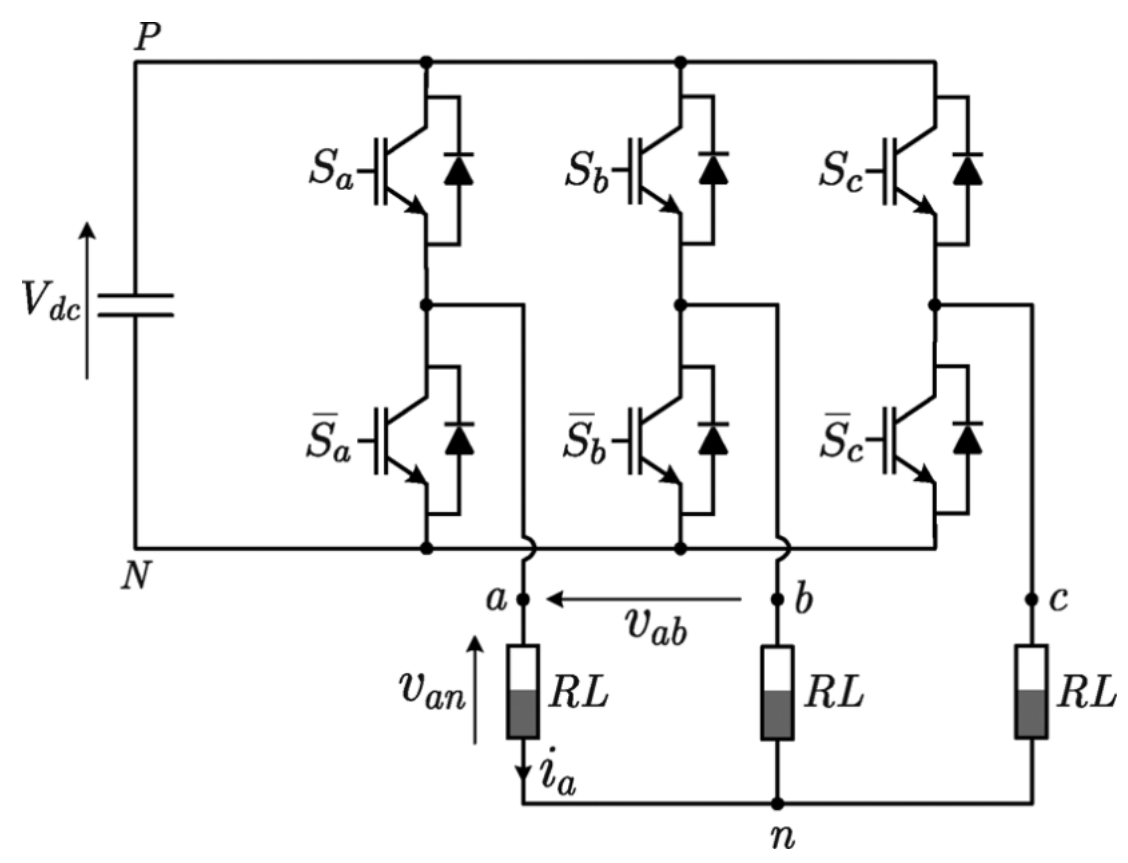

Figure 2-9: A general three-phase DG inverter configuration with RL load [93].

To formulate the eight control sets, the switching signals can be represented as follows:

$$
\begin{aligned}
& S_{1}=\left\{\begin{array}{l}
1, \text { if } S_{a}=1 \text { and } \bar{S}_{a}=0 ; \\
0, \text { if } S_{a}=0 \text { and } \bar{S}_{a}=1 ;
\end{array}\right. \\
& S_{2}=\left\{\begin{array}{l}
1, \text { if } S_{b}=1 \text { and } \bar{S}_{b}=0 ; \\
0, \text { if } S_{b}=0 \text { and } \bar{S}_{b}=1 ;
\end{array}\right. \\
& S_{3}=\left\{\begin{array}{l}
1, \text { if } S_{c}=1 \text { and } \bar{S}_{c}=0 ; \\
0, \text { if } S_{c}=0 \text { and } \bar{S}_{c}=1 ;
\end{array}\right.
\end{aligned}
$$

where 1 represents the closed state of a switch, and 0 represents the opened state of a switch. Transforming into a vectorial form, the switching signals can then be expressed as follows:

$$
\mathbf{S}=\frac{2}{3}\left(S_{1}+\boldsymbol{\alpha} S_{2}+\boldsymbol{\alpha}^{2} S_{3}\right)
$$

where $\boldsymbol{\alpha}=e^{j \frac{2 \pi}{3}}$, and $\mathbf{S}$ is the switching vector for the DG inverter. Therefore, the output voltage of the DG inverter can then be expressed as follows with (2.12): 


$$
\mathbf{v}_{D G}=V_{d c} \mathbf{S}
$$

where $\mathbf{v}_{D G}$ is the output voltage of the DG inverter, and $V_{d c}$ is the DC voltage of the DG unit. Formulating the control problem into such a finite control set problem can greatly reduce the computational requirement of the MPC algorithm. The DG inverter will then be modeled using Kirchhoff's voltage and current laws, and a cost function will be defined to control the preferred system state variables.

Many research works have been published regarding the FCS-MPC. A fixed switching frequency scheme is proposed for the FCS-MPC in [94]. The algorithm proposed divides the algorithm into two parts: the offline calculation and evaluation of the coefficients of the equations, and the online monitoring of the controller. The power quality of the output of the DG inverter can still be guaranteed, compared to PWM generators. In [95], an FCS-MPC algorithm for the DG inverter with an LCL filter is proposed. The authors compare different approaches of using the FCS-MPC, such as using inverter internal equivalent current feedback and using inverter output current feedback. The proposed FCS-MPC algorithm can provide high dynamic performance and potential additional functions, such as multivariable control, reduced switching losses and low harmonic distortion. Also in [96], an effective method to simplify the FCS-MPC is proposed. Because of the use of equivalent transformation and the specialized sector distribution method, the proposed method can greatly reduce the computational requirement of the FCS-MPC without affecting the control performance.

The implementation of MPC algorithm in inverter control is usually neglected because of its complexity in computation. [94]-[96] focuses more on how to use simplified MPC algorithm to reduce such computational burden, and advanced control strategies are not found in them.

\subsection{Operation and Control Strategy in Microgrid EMS}

Aside from the abovementioned different control algorithms in controlling local inverters or optimizing the operation of the microgrid, another important aspect which 
significantly affects the microgrid is the operation and control strategy which is integrated in microgrid EMS. These strategies define the operating principles of all the DG units and loads in microgrids to sustain the operation.

\subsubsection{Operation and Control Strategy Diagram}

Control strategy is usually in the form of a diagram as a flowchart of different actions. Similar to Figure 2-5, many research works have been published to propose different operation and control strategies in the microgrid EMS. In [97], an operation and control strategy is proposed in a microgrid consisting of WT, diesel generator, ESS and a sea water desalination system as a flexible load. This control strategy uses hourly wind speed forecast data, to maximize the utilization of wind energy using the ESS. Figure 2-10 explains the operation and control strategy for both the ESS and the WT. In the system, the authors define the SOC of the ESS into four demarcation points, maximum SOC $\left(S O C_{\mathrm{max}}\right)$, SOC high point $\left(S O C_{\mathrm{high}}\right)$, SOC low point $\left(S O C_{\text {low }}\right)$ and minimum SOC $\left(S O C_{\min }\right)$ where $100 \%>S O C_{\max }>S O C_{\text {high }}>S O C_{\text {low }}>S O C_{\min }>0 \%$. The operation of the microgrid includes two major operating modes: WT/diesel generator/battery mode (WDBM) and WT/battery mode (WBM). From Figure 2-10, the operation and control strategy is adjusted every 10min according to the SOC of ESS. The EMS will adjust the operating modes as well as the ON/OFF status of the units within the desalination system accordingly. 


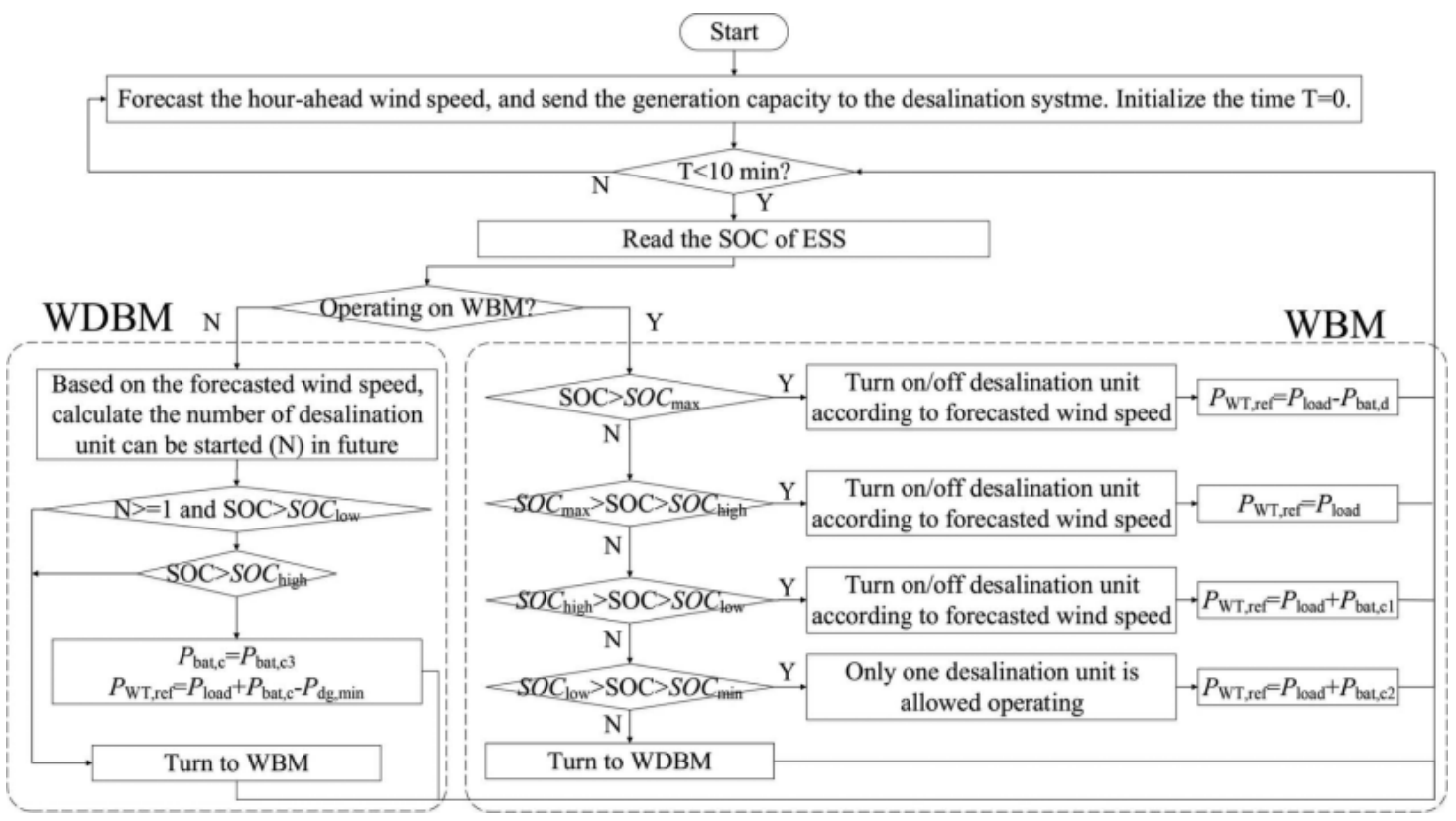

Figure 2-10: Operation and control strategy proposed in [97].

In [98], an operation and management strategy for an islanded microgrid consisting of a primary DG unit and a PV/battery system is proposed. Integrating a proposed power flow control and a DG inverter control, the proposed control strategy is illustrated in Figure 2-11. It is clear that the strategy is based on the SOC of the battery, similar to the aforementioned paper. The SOC of the battery is divided into three demarcation points, maximum SOC $\left(S O C_{\max }\right)$, a predefined limit $\left(\overline{S O C}_{\max }\right)$, and minimum SOC $\left(S O C_{\min }\right)$. When the SOC is smaller than SOCmin, the priority to charge the battery is higher than the priority for power sharing. When the $\mathrm{SOC}$ is between $S O C_{\min }$ and $S O C_{\max }$, the PV will take responsibility to share power within the microgrid while the excessive power will be stored in the battery. When the SOC is between $\overline{S O C}_{\max }$ and $S O C_{\max }$, the PV/battery system will supply maximum available power to the microgrid as long as the load demand is high enough; otherwise, the generation of this system will match the load by adjusting the battery. When the SOC is larger than $S O C_{\max }$, the PV will not operate in maximum power point tracking (MPPT) mode. The excessive power generated by the PV will be curtailed using the proposed inverter control. The proposed operation and control strategy utilize multi-loop control and multi-segment adaptive droop control without relying on communications or a state machine. 


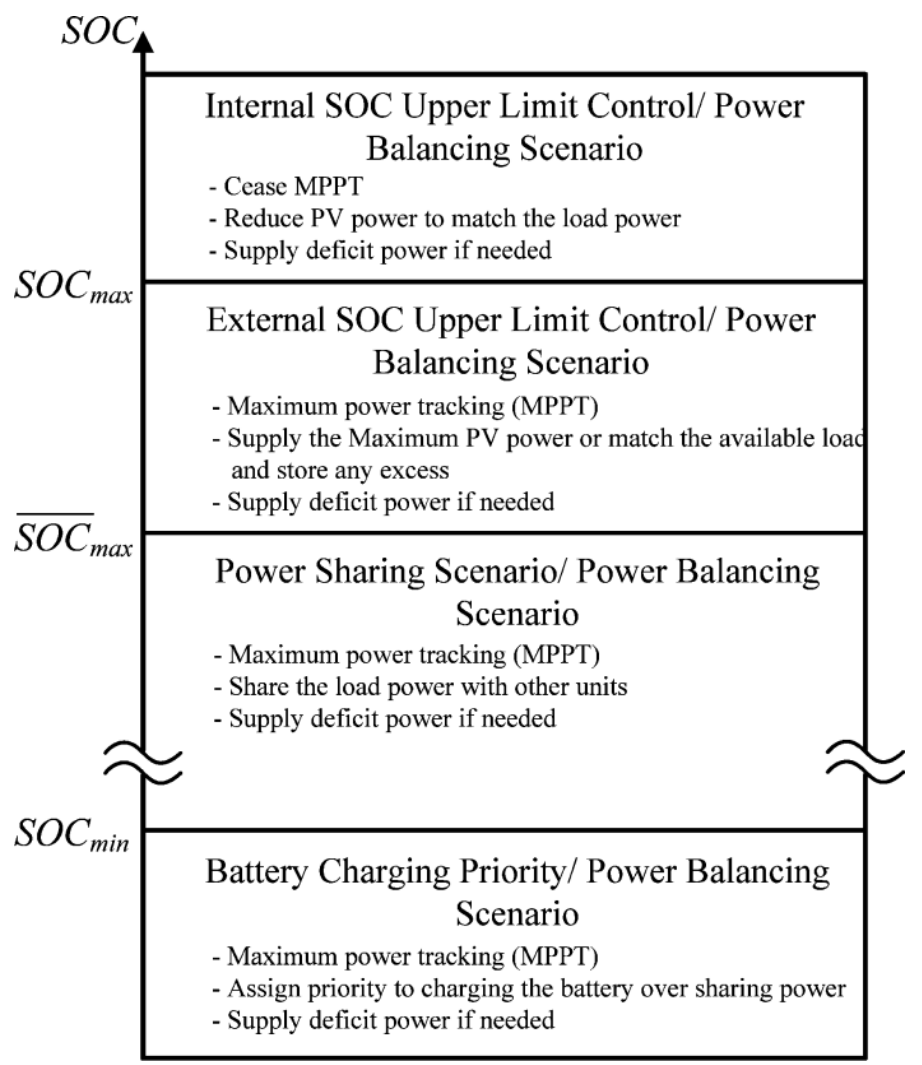

Figure 2-11: Operation and control strategy for a PV/battery system [98].

Similarly, in [70], the operation and control algorithm proposed for islanded microgrids, which includes RESs and ESSs, is shown in Figure 2-12. It is rather straight forward since the authors propose to use the droop based control algorithm.

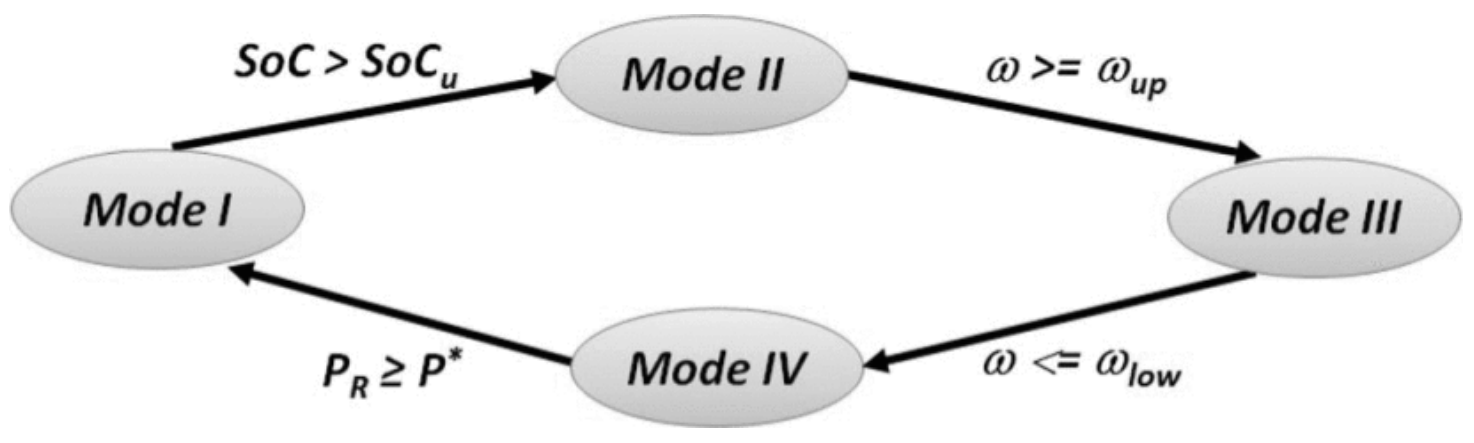

Figure 2-12: Operation and control strategy proposed in [70].

The control strategy is based on the conditions of the SOC of the ESSs and the frequency of the islanded microgrid. In Figure 2-12, Mode I refers to the mode when at least one ESS is working in VCM while all RESs are working in power control 
mode (PCM) which is basically CCM. Mode II refers to the mode when all the ESSs and RESs are working in PCM. Mode III refers to the mode when at least one RES is working in VCM while the ESSs are working in PCM. Mode IV refers to the mode when all the ESSs and RESs are working in PCM.

\subsubsection{Load Shedding Strategy}

In the operation and control strategies in microgrid EMS, load control is also one of the most important aspects of microgrid operation. From [97], the control strategy includes the control of the flexible load of desalination units. However, flexible loads cannot always be found in microgrids, and the most common method to control the load is through load shedding.

Load shedding is an energy management technique already applied in traditional power systems [99], [100]. Load shedding is usually activated during extreme conditions when total generation cannot meet load demand. However, nowadays power systems adopt automatic load shedding schemes based on system frequency or bus voltage. In [101], under-voltage load shedding is discussed. A proposed undervoltage load shedding scheme for a $500-\mathrm{kV}$ bus line is proposed as follows:

- $5 \%$ of the load is shed at a voltage $10 \%$ below the lowest allowed voltage with a $1.5 \mathrm{~s}$ delay;

- $5 \%$ of the load is shed at a voltage $8 \%$ below the lowest allowed voltage with a 3s delay;

- $5 \%$ of the load is shed at a voltage $8 \%$ below the lowest allowed voltage with a 6 delay.

From the proposed scheme, it is observed that the load will be automatically shed at the bus line according to the voltage level, and the objective is to restore the bus voltage back to its allowed range.

The Israel Electric Corporation's automatic under-frequency load shedding can be found in [102], which is illustrated in Table 2-1. From Table 2-1, there are 18 stages in total for the load shedding scheme, with each stage shedding a certain amount of load 
according to historical data which contains approximately 30 cases of forced outages of large generating units that activate the load shedding system per year. (N.W.C. refers to the national water carrier which is basically water pumps that consume 90MW of real power.)

Table 2-1: Israel Electric Corporation Under-Frequency Load Shedding Scheme [102]

\begin{tabular}{|c|c|c|c|}
\hline Stage No. & Frequency $(\mathrm{Hz})$ & Delay (s) & Load (\%) \\
\hline 1 & 49.4 & $\mathrm{~d} f / \mathrm{d} t=-0.4$ & 3.1 \\
\hline 2 & 49.4 & 0.2 & N.W.C \\
\hline 3 & 49.4 & 0.5 & N.W.C \\
\hline 4 & 49.0 & 0.2 & 5.5 \\
\hline 5 & 49.0 & 0.4 & 1.5 \\
\hline 6 & 48.8 & 0.2 & 3.8 \\
\hline 7 & 48.8 & 0.4 & 1.5 \\
\hline 8 & 48.6 & 0.3 & 4.0 \\
\hline 9 & 48.6 & 0.6 & 1.1 \\
\hline 10 & 48.6 & 0.8 & 5.5 \\
\hline 11 & 48.6 & 1.0 & 1.4 \\
\hline 12 & 48.2 & 0.3 & 1.2 \\
\hline 13 & 48.2 & 0.4 & 8.8 \\
\hline 14 & 48.2 & 1.0 & 3.8 \\
\hline 15 & 48.2 & 0.4 & 0.4 \\
\hline 16 & 48.0 & 1.0 & 2.3 \\
\hline 17 & 47.8 & 0.5 & 9.1 \\
\hline 18 & 47.8 & 1.0 & 1.7 \\
\hline
\end{tabular}

From Table 2-1, the amount of load to be shed at each stage usually relies on the historical data in the power system. These two load shedding schemes, i.e., undervoltage load shedding and under-frequency load shedding, both use historical data to determine the load to be shed at each stage. This can reduce the calculation while guaranteeing the voltage or frequency is restored back to its nominal operating range. However, it will be impossible for new systems to have historical data which can be 
used as references. Therefore, there are research works [103], [104] which use realtime system states of all DG units to calculate the amount of load that needs to be shed.

For microgrids, load shedding is usually activated due to economic optimization or, like traditional power systems, extreme conditions when load demand cannot be met by total power generation. Aside from adapting the existing industrial methods mentioned above, new methods have been proposed to solve unique situations in microgrids with DG units. In [105], a dynamic load shedding strategy for an islanded microgrid with limited generation capability from the DG units is proposed. This strategy is based on the Markov decision process which can obtain the optimal load shedding strategy that has the maximum expected economic benefit. A new method to use nonlinear programing to optimize the under-frequency load shedding strategy in a microgrid is proposed in [106]. Generic algorithm is used to minimize the amount of load to be shed and guarantees the lowest frequency deviation. Tree-encoding chromosomes are incorporated to ensure the load shedding amounts reduce gradually from the first stage to the last stage. The probability of each possible scenario is considered in the proposed load shedding scheme. In [107], a centralized underfrequency load shedding strategy is proposed with several control modules, including an event calculator module (ECM), a frequency calculator module (FCM), a distribution state estimator module (DSEM) and a load shedding controller module (LSCM). The ECM will monitor the real-time measurements, detect the status of the microgrid and send the data and even report to the FCM, DSEM, and LSCM. The FCM calculates the frequency based on synchronized measurements of the grid frequency, and sends it to the LSCM. The DSEM estimates the load demand at all the buses in the microgrid. The weighted least square algorithm is used. The LSCM calculates the power imbalance in the microgrid. By using this method, the microgrid can sustain operation during different scenarios by adjusting the amount of load to be shed.

\subsection{Network Configuration in DC Based Microgrids}

In AC microgrids, DG units are connected to microgrid mostly through DG inverters, together with other converters. Therefore, even though the configuration of a microgrid network can be different from another, the operation and control of the 
microgrid in the local controllers and the microgrid EMS can still be similar. However, in DC microgrids, different configurations, especially in terms of the DG inverters, can result in totally different operation and control strategies.

In [108], a DC microgrid based wind farm architecture, in which each wind energy conversion unit consists of a matrix converter, a high frequency transformer and a single-phase rectifier, is proposed. In addition, all the wind energy conversion units are connected to a three-phase AC output through a single inverter. The system can be seen in Figure 2-13. It is observed that this proposed topology uses only one cable for each wind energy conversion unit to be connected to the AC network. However, it is very risky to use only one inverter to connect many WTs to the AC network due to reliability issues.

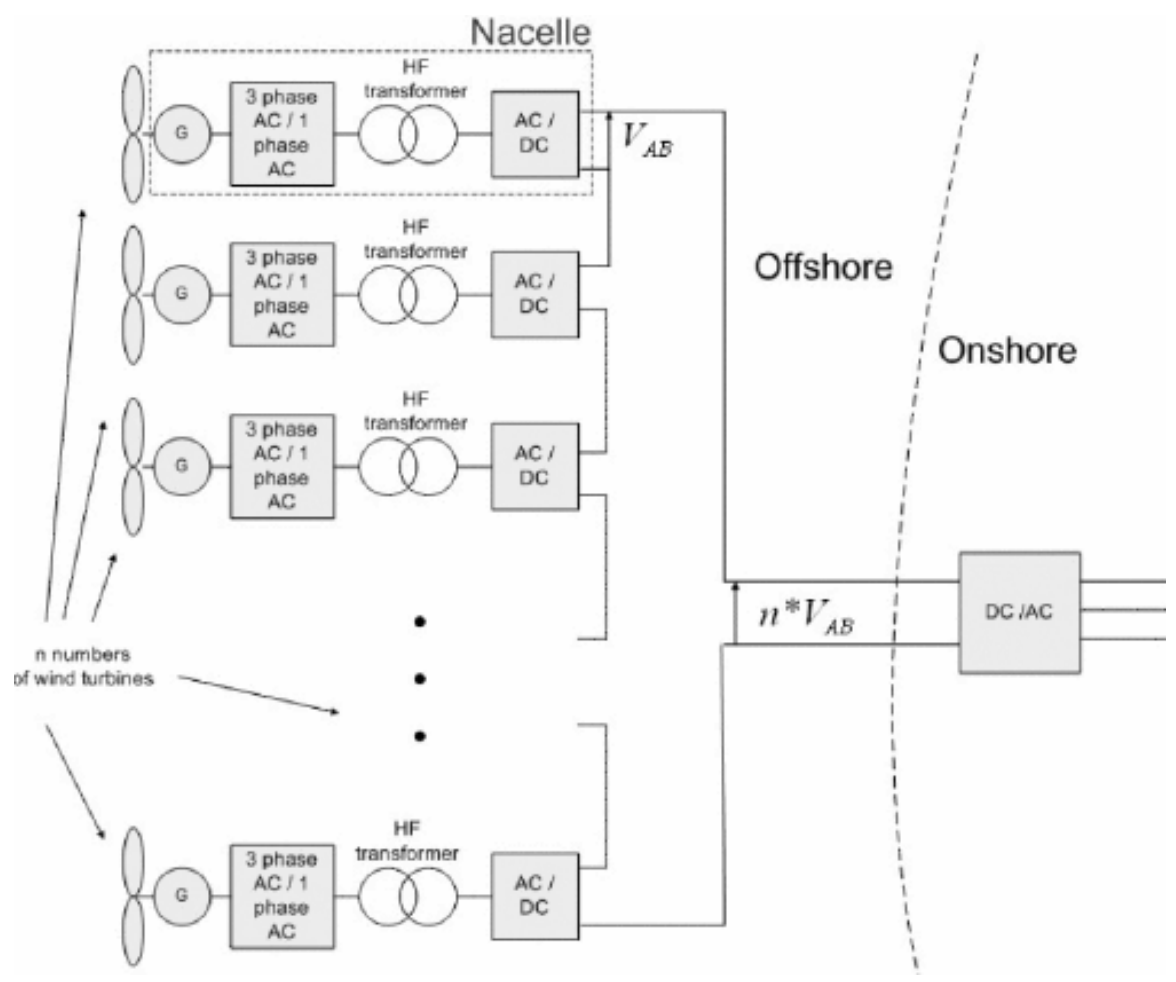

Figure 2-13: DC microgrid network proposed in [108]

Similarly, in [109], a DC microgrid based wind farm architecture, in which the WTs are clustered into groups of four with each group connected to a rectifier, is proposed. All the groups are then connected to the AC network through one inverter. With the proposed architecture, the failure of one rectifier will result in all four WTs of the 
same group to be out of service, and the failure of the inverter will result in all the groups to be out of service.

In [110], a hybrid AC/DC grid architecture that consists of both $\mathrm{AC}$ and DC networks connected by a bidirectional converter is proposed as shown in Figure 2-14. Hierarchical control algorithms are incorporated to ensure smooth power transfer between the AC microgrid and the DC microgrid under various operating conditions. However, failure of the bidirectional converter will result in the isolation of the DC microgrid from the AC microgrid.

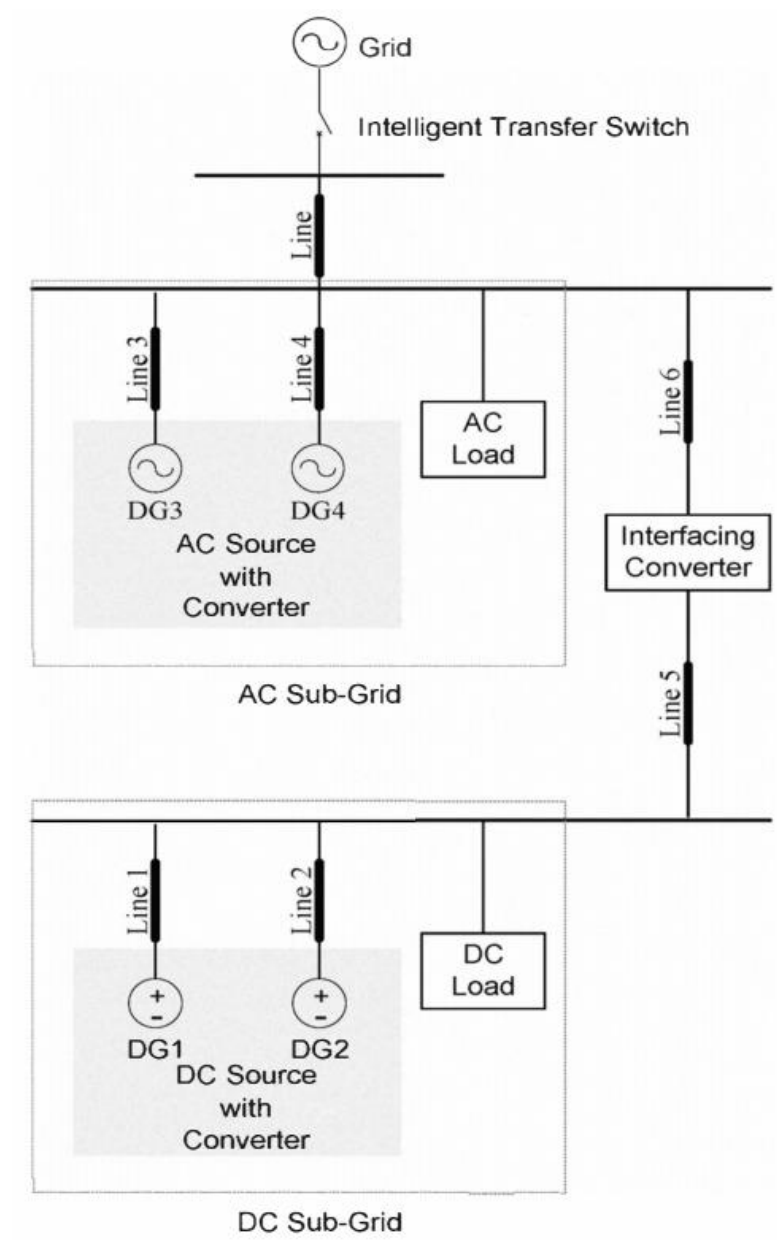

Figure 2-14: Hybrid DC/AC microgrid configuration proposed in [110]

It can be noticed from the above research works [108]-[110], conventional DC network configurations can be categorized into two groups, i.e., one-inverter-one-DG 
and one-inverter-multiple-DG. However, the former one suffers from large burden in cost, while the latter one reduces the system reliability drastically.

\subsection{Summary}

From the above literature review, it is indicated that inverter operation in a microgrid is incorporated in all aspects of operation and control of the microgrid. Whether in a centralized or decentralized approach in the operation of the microgrid, the control algorithm and operating strategy of the DG inverter need to be addressed. Although the emphasis on inverter operation is more on the decentralized approach than the centralized approach, there are many important aspects still controlling the DG inverter in the centralized approach in terms of power quality and system stability. For both grid-connected and islanded conditions, a DG inverter is to be controlled in different working modes to sustain the operation of the microgrid. This thesis focuses on both operating conditions to ensure the proposed control strategies can efficiently handle the two conditions. In terms of power balance, real power management and reactive power compensation should be considered in the DG inverter control, and droop control strategies are commonly used for the operation and coordination in real and reactive power sharing between the DG inverters. System voltage and frequency stability relies on the operation of the EMS as well as the local DG inverter controllers. Traditional local inverter control algorithms, such as PI, PR, etc., can attain the performance requirements, but advanced control algorithms, such as MPC, can improve the performance and provide additional functions, such as harmonic compensation. These advanced control algorithms can be utilized in a centralized approach or a decentralized approach. Apart from the local DG inverter control algorithms, the operation and control strategies in the microgrid EMS are crucial in managing the operation of the DG inverters. With a proper control strategy, the DG inverters can operate not only continuously, but also economically. Additionally, load control strategies, such as load shedding, can be implemented together with the operation and control of DG inverters to further improve system reliability. Typical load shedding schemes are very complicated, and they are required to be adjusted for better implementation in microgrids. Therefore, a combined centralized and decentralized approach for the DG inverters in a microgrid with operation and control strategies and advanced local inverter control algorithms can guarantee system 
stability and reliability. And this thesis investigates further in the combined centralized and decentralized approach in the operation. In particular, for a DC microgrid, the configuration of the DG inverters will affect the operation of the microgrid. Thus, choosing the proper DG inverter configuration is equally important as well as designing operation and control strategies and local inverter control algorithms for the DC microgrid. This thesis investigates the network configuration of the DC microgrid. The proposed DC microgrid configuration can increase the reliability and reduce the cost compared to a conventional DC microgrid configuration. 


\section{CHAPTER 3 CENTRALIZED CONTROL FOR PARALLEL OPERATION OF DISTRIBUTED GENERATION INVERTERS IN MICROGRIDS [111]}

\section{Nomenclature}

$P_{j}$

$Q_{j}$

$P_{L}$

$Q_{L}$

$u_{j}$

$u_{s}$

$u_{t}$

$V_{d c j}$

$L_{f j}$

$C_{f j}$

$R_{f j}$

$i_{L j}$

$i_{L f j}$

$i_{L h j}$

$I_{L f j}$

$I_{L h j}$

$\varphi_{L f j}$

$\varphi_{L h j}$

$v_{G}$

$i_{L f j, p}$

$i_{L f j, q}$

$i_{D G j}$

$v_{D G j}$

$i_{G j}$

$R_{l j}$

$L_{l j}$

$i_{j}$

$\omega_{j}$

Real power delivered by the DG and $j=1,2,3,4$.

Reactive power delivered by the DG and $j=1,2,3,4$.

Real power consumption of the load.

Reactive power consumption of the load.

Control signal for the DG inverter and $j=1,2,3,4$.

Steady-state control signal.

Transient control signal.

DC link voltage of the DG inverter and $j=1,2,3,4$.

Inductance of the LC filter in the DG inverter and $j=1,2,3,4$.

Capacitance of the LC filter in the DG inverter and $j=1,2,3,4$.

Loss of the DG inverter and $j=1,2,3,4$.

Load current and $j=1,2$.

Fundamental component of load current and $j=1,2$.

Harmonic component of load current and $j=1,2$.

Peak amplitude of fundamental component of load current and $j=1,2$.

Peak amplitude of harmonic component of load current and $j=1,2$.

Phase angle of the fundamental components of $i_{L j}$.

Phase angle of the harmonic components of $i_{L j}$.

Distribution grid voltage

Instantaneous fundamental phase component of $i_{L f j}$.

Instantaneous quadrature phase component of $i_{L f j}$.

Output current of the DG inverter and $j=1,2,3,4$.

Output voltage of the DG inverter and $j=1,2,3,4$.

Grid current at the PCC and $j=1,2$.

Line resistance from the microgrid to the upstream utility substation.

Line inductance from the microgrid to the upstream utility substation.

Current passing through $L_{f j}$ and $j=1,2,3,4$.

Frequency of the output voltage and $j=1,2,3,4$. 


$\begin{array}{ll}k_{P, j} & \text { Droop characteristics for real power and } j=1,2,3,4 . \\ k_{Q, j} & \text { Droop characteristics for reactive power and } j=1,2,3,4 . \\ P_{r e f, j} & \text { Real power reference of droop characteristics and } j=1,2,3,4 . \\ Q_{r e f, j} & \text { Reactive power reference of droop characteristics and } j=1,2,3,4 . \\ e_{s} & \text { Defined tracking error for optimization. }\end{array}$

\subsection{Introduction}

This chapter presents a centralized operation and control strategy with an MPC local inverter control algorithm that enables the parallel operation of DG inverters in a microgrid. The newly developed MPC algorithm transforms the control problem into two subproblems that refer to the steady-state subproblem and the transient subproblem. By separating and solving these two subproblems, reduced computational burden can be achieved as well as harmonic compensation. The proposed operation and control strategy in microgrid EMS coordinates the operation of DG inverter power sharing and load in both grid-connected and islanded conditions. Several simulation studies of different test scenarios are conducted to evaluate the proposed operation and control strategy and local control algorithm. The simulation results prove the effectiveness of the proposed operation and control strategy and the MPC algorithm in maintaining the microgrid operation under different situations.

\section{2 $\quad$ System Description and Modeling}

\subsubsection{System Description}

The proposed configuration of the microgrid, which can both work in grid-connected and islanded conditions, is shown in Figure 3-1. Within this microgrid are contained several components: an MT with a power capacity of $15 \mathrm{kVA}$, a PV array with a power capacity of $5 \mathrm{kWp}$, a Li-ion SB with an energy capacity of $20 \mathrm{Ah}$, and a PEMFC with a power capacity of $5 \mathrm{~kW}$. The PV array operates as a main DG unit that supplies power whenever solar irradiance exists; the MT also operates as a main DG unit in the microgrid. The SB and the PEMFC stack operate as back-up DG units both for the PV array and for the power shortage, in general, for the microgrid. The SB can 
be charged during off-peak hours by the distribution grid when the electricity price is low. Alternatively, the SB can delivery power to the microgrid during peak hours for peak shaving.

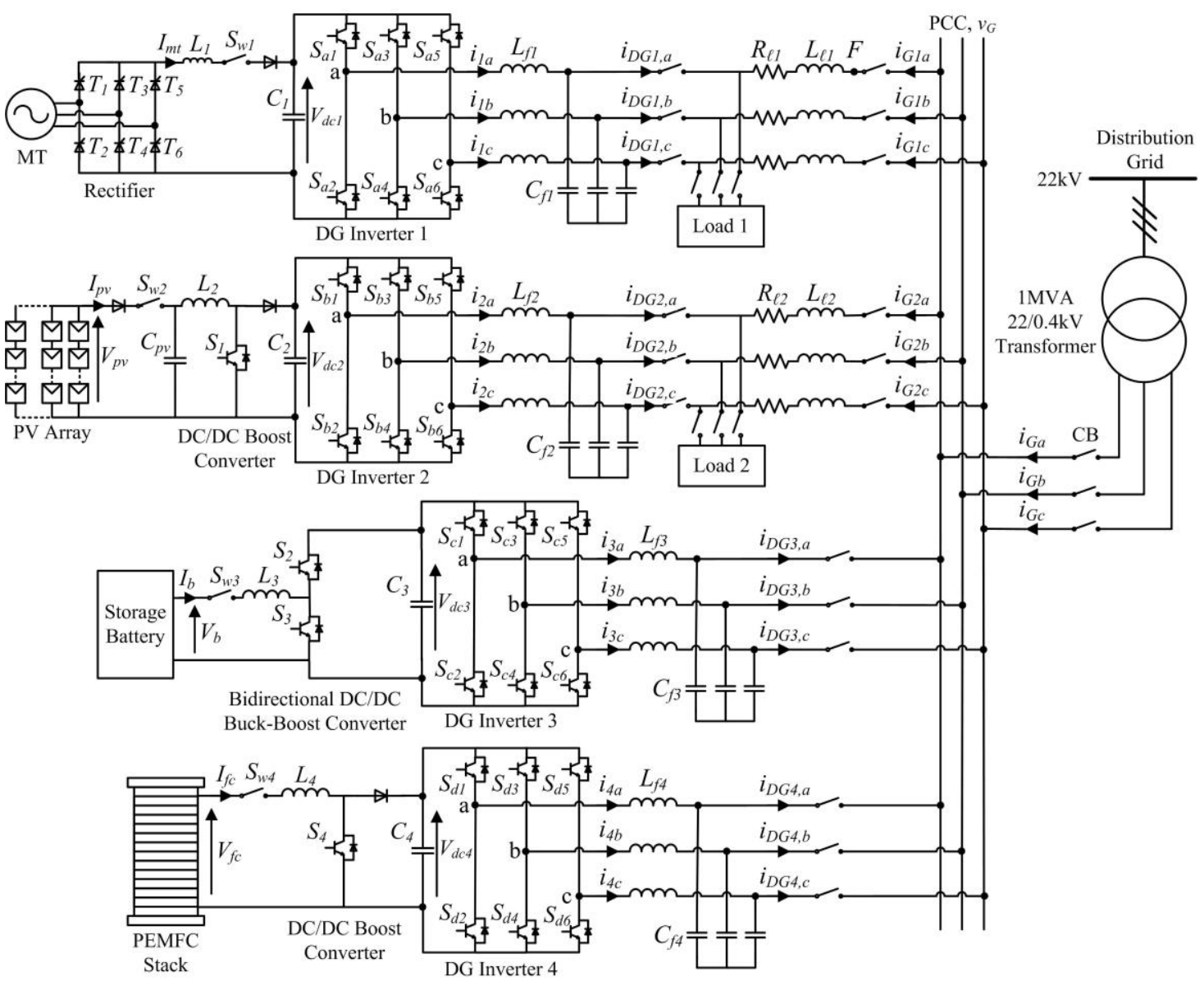

Figure 3-1: Overall configuration of the proposed microgrid architecture.

The distribution grid is connected to the microgrid at the PCC through a circuit breaker (CB) in grid-connected condition. The main DG units (i.e., the PV array and the MT) deliver real and reactive power and provide voltage support. This can reduce the stress of the distribution grid in terms of power generation and power delivery. In terms of harmonic distortion of load currents caused by the increase of power electronics equipment in the load, the DG units also can provide harmonic compensation in the load currents. By doing so, these harmonics will not affect the upstream distribution network that is connected to the PCC. 
The microgrid will operate in islanded condition when the $\mathrm{CB}$ cuts the connection between the microgrid and the distribution grid when a fault occurs in the upstream distribution network. This leaves the DG units as the sole power sources for the microgrid. If the main DG units are not able to cover the load demand of the microgrid, the back-up DG units (i.e., the SB and the PEMFC stack) will be activated to deliver the required power, including real power and reactive power, to the microgrid to maintain its operation.

\subsubsection{Grid Operation}

All the dc-link voltages of the 4 DG units are regulated by their respective rectifier, $\mathrm{dc} / \mathrm{dc}$ boost converter, and bidirectional dc/dc buck-boost converter, as shown in Figure 3-1. The respective DG unit is assumed to deliver the necessary power to the dc-link to achieve power balance.

During grid-connected condition, the distribution grid is maintaining the power balance of the overall distribution system. Nonetheless, if the microgrid operates in islanded condition, the power balance in the microgrid is regulated by the SB and the PEMFC stack according to the power balance equation as follows:

$$
P_{m t}+P_{p v}+P_{b}+P_{f c}=P_{L}
$$

where $P_{m t}$ and $P_{p v}$ are the real power delivered by the MT and the PV array, respectively, $P_{b}$ is the real power delivered by the SB that is subject to

$$
P_{b} \leq\left|P_{b, \max }\right|
$$

$P_{f_{c}}$ represents the real power delivered by the PEMFC stack, and $P_{L}$ represents the real power consumption of the load. The SB has its constraints in terms of the stored energy that are related to the SOC that can be expressed as:

$$
S O C_{\min } \leq S O C \leq S O C_{\max }
$$


Currently, the SOC of the SB cannot be directly measured, yet several methods [112][114] can be used to estimate it. During grid-connected condition, the SB can be charged during off-peak hours if its SOC is below $S O C_{\max }$. During islanded condition, the SB can be charged, discharged or in idle depending on its SOC. A droop control method, which will be further illustrated in Section 3.2.4, is used to ensure that the load consumption is shared appropriately. The flowcharts in Figures 3-2 and 3-3 illustrate the operations of the SB and the PEMFC stack during grid-connected and islanded conditions, respectively.

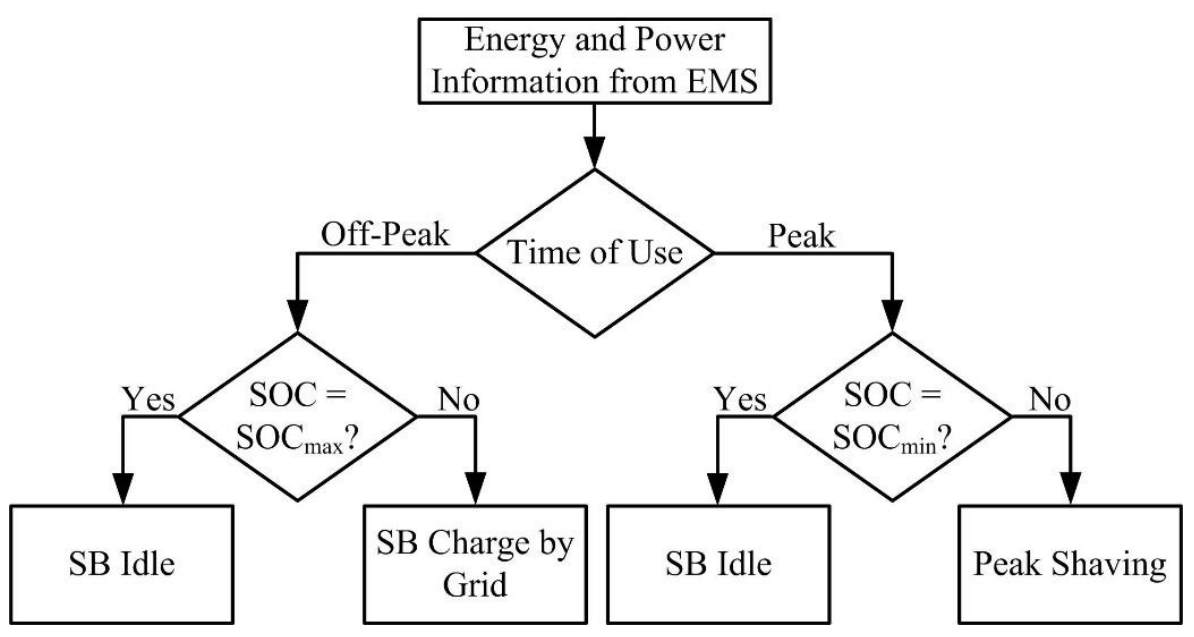

Figure 3-2: Operation of the SB during grid-connected condition. 


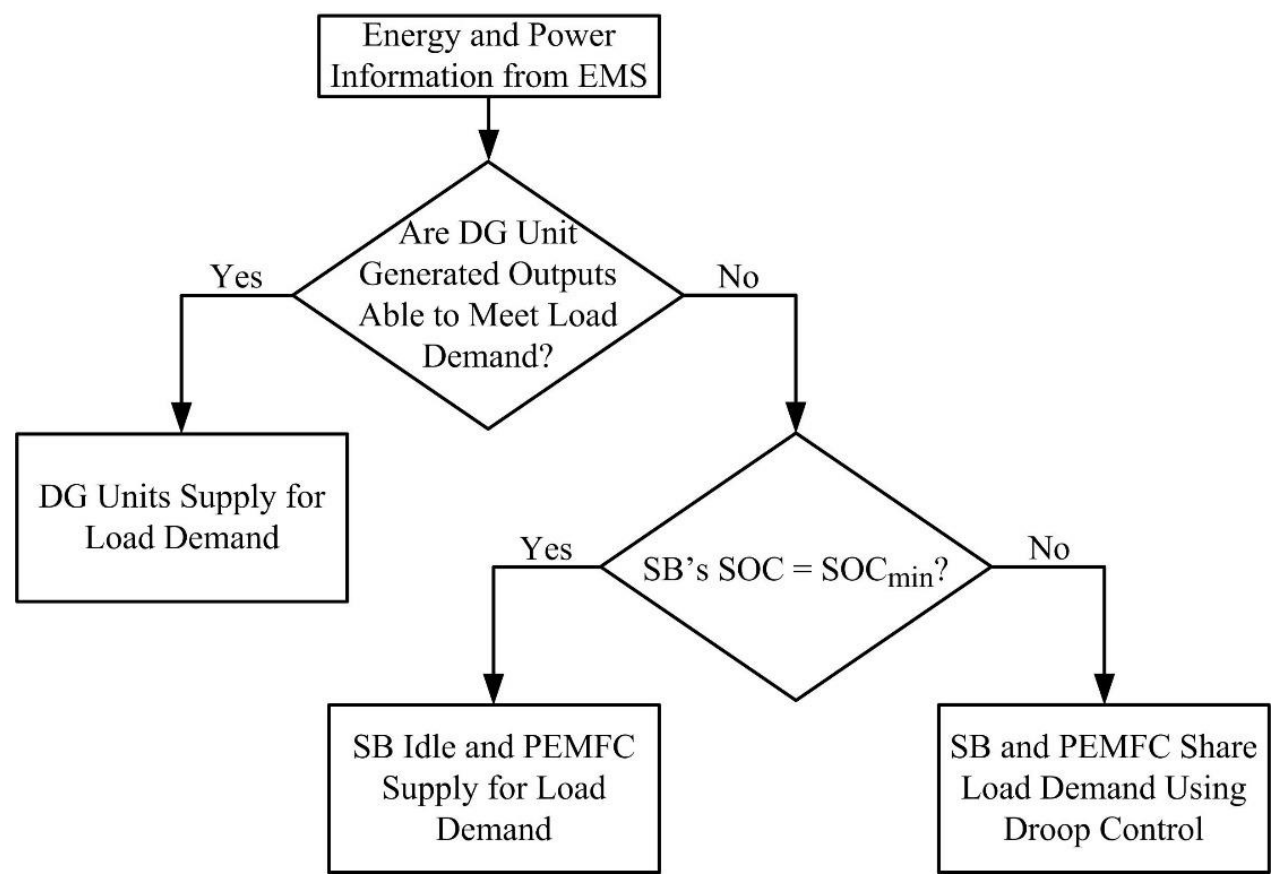

Figure 3-3: Operation of the SB and PEMFC stack during islanded condition.

\subsubsection{DG Inverter Modeling}

During grid-connected condition, the single-phase representation of the DG inverter of the MT or PV array can be illustrated in Figure 3-4. Additionally, the DG inverters of the SB and the PEMFC stack can be illustrated in Figure 3-5 as the single-phase representation during islanded condition [80], [115], [116].

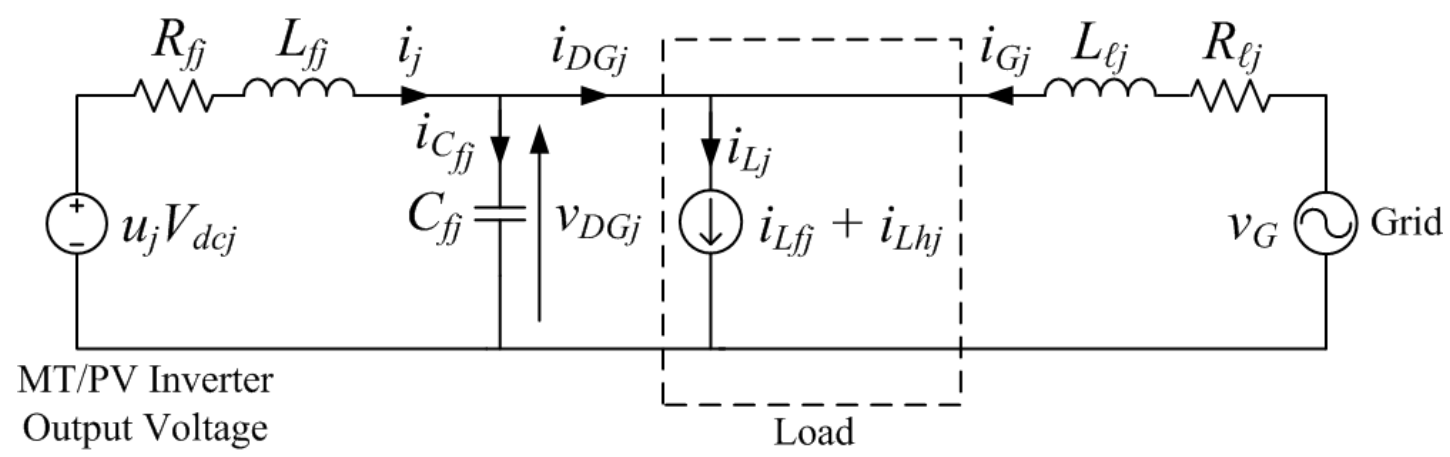

Figure 3-4: Equivalent single-phase representation of the DG inverter of MT $(j=1)$ or $\mathrm{PV}$ array $(j=2)$ during grid-connected condition. 


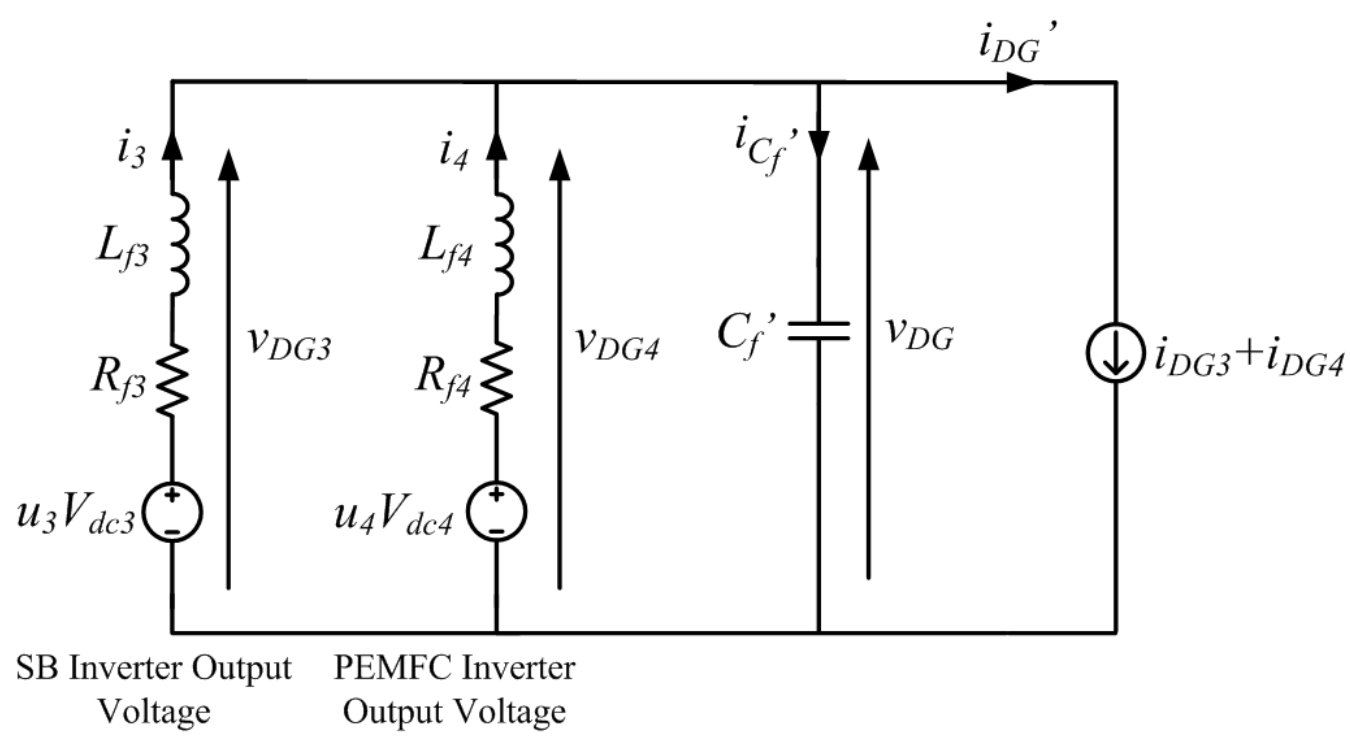

Figure 3-5: Equivalent single-phase representation of the DG inverters of the SB ( $j=3)$ and the PEMFC stack $(j=4)$ during islanded condition.

The controlled voltage, which is the output of the DG inverter, is represented as $u_{j} V_{d c j}$, where $u_{j}$ is the control signal and $j=1,2,3,4$. As shown in Figure 3-4, an LC filter, which is given by $L_{f j}$ and $C_{f j}$, is added after the DG inverter. This is to eliminate the high switching frequency harmonics from the DG inverter. The loss of the DG inverter is modeled as a resistance $R_{f j}$. The load current $i_{L j}(j=1,2)$ is represented by two parts (i.e., the fundamental $i_{L f j}$ and the harmonic $i_{L h j}$ ) which have peak amplitudes of $I_{L f j}$ and $I_{L h j}$ respectively:

$$
\begin{aligned}
i_{L j} & =i_{L f j}+i_{L h j}=I_{L f j} \sin \left(\omega t-\varphi_{L f j}\right)+\sum_{h=3,5, \ldots}^{N} I_{L h j} \sin \left(h \omega t-\varphi_{L h j}\right) \\
& =I_{L f j} \sin (\omega t) \cos \left(\varphi_{L f j}\right)-I_{L f j} \cos (\omega t) \sin \left(\varphi_{L f j}\right)+\sum_{h=3,5, \ldots}^{N} I_{L h j} \sin \left(h \omega t-\varphi_{L h j}\right) \\
& =i_{L f j, p}+i_{L f j, q}+i_{L h j}
\end{aligned}
$$

where $\varphi_{L f j}$ and $\varphi_{L h j}$ represent the phase angles of the fundamental and the harmonic parts of $i_{L j}$, respectively, with respect to the phase angle of the distribution grid voltage $v_{G}$, and $i_{L f j, p}$ and $i_{L f j, q}$ are the instantaneous fundamental phase and quadrature components of $i_{L f j}$. The output current $i_{D G}$ of the DG inverter of the MT or PV array is 
set to maintain unit power factor at the PCC, compensate for the harmonics of the load currents, and achieve load sharing simultaneously. Therefore, these output currents can be summarized as follows:

$$
i_{D G j}=\left(i_{L f j, p}-i_{G j}\right)+i_{L f j, q}+i_{L h j}
$$

where $i_{G j}(j=1,2)$ is the current of the grid at the PCC. As shown in Figure 3-4, the voltage source $v_{G}$ which represents a utility substation is linked to the microgrid and load through a line resistance $R_{l j}$ and line inductance $L_{l j}$ during grid-connected condition. During grid-connected condition, the DG inverters in the microgrid share the load consumption, and the grid voltage is certain. Therefore, CCM is applied to the DG inverter of the MT or the PV array to regulate the output current. AS shown in Figure 3-5, during islanded condition, the output voltage is required to be regulated to a sine wave with a fixed magnitude, which can be achieved through VCM.

As shown in Figure 3-6, the state-space model for the DG inverters could be derived using Kirchhoff's voltage and current laws:

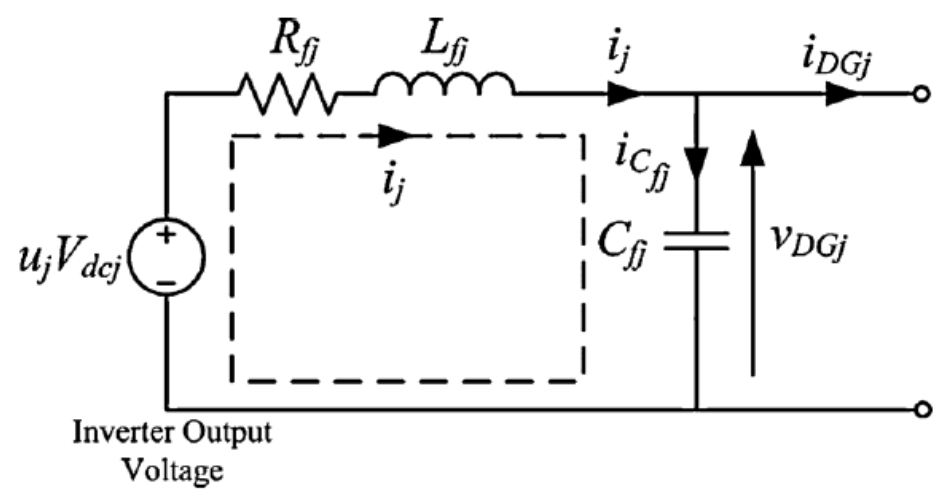

Figure 3-6: Single-phase representation of the DG inverter during both grid-connected and islanded conditions.

$$
\begin{gathered}
\frac{d i_{j}}{d t}=-\frac{R_{j}}{L_{f j}} i_{j}-\frac{1}{L_{f j}} v_{D G j}+\frac{V_{d c j}}{L_{f j}} u_{j} \\
\frac{d v_{D G j}}{d t}=-\frac{1}{C_{f j}} i_{j}-\frac{1}{C_{f j}} i_{D G j}
\end{gathered}
$$


where $i_{j}$ is the current that passes through $L_{f j}$. Therefore, the DG inverter model during grid-connected condition can be written as follows:

$$
\begin{gathered}
\dot{x}_{g j}=A_{g j} x_{g j}+B_{g j 1} v_{j}^{\prime}+B_{g j 2} u_{j} \\
y_{g j}=C_{g j} x_{g j}+D_{g j 1} v_{j}^{\prime}+D_{g j 2} u_{j}
\end{gathered}
$$

where the subscripts $g$ and $j$ are the models of the DG inverter $j$ during grid-connected condition $(j=1,2)$, and $A_{g j}=-\frac{R_{j}}{L_{f j}} ; B_{g j 1}=\left[-\frac{1}{L_{f j}} 0\right] ; B_{g j 2}=\frac{V_{d c j}}{L_{f j}} ; C_{g j}=1 ;$ $D_{g j 1}=\left[\begin{array}{ll}0 & -C_{f j}\end{array}\right] ; D_{g j 2}=0 ; x_{g j}=i_{j}$ is the state; $v_{j}^{\prime}=\left[v_{D G j} \frac{d v_{D G j}}{d t}\right]^{T}$ is the exogenous input; $u_{j}$ is the control signal with $-1 \leq u_{j} \leq 1$; and $y_{g j}=i_{D G j}$ is the output, which will be regulated according to reference signals. In the simulation studies in this chapter, DG inverters 3 and 4 are not used during grid-connected condition. However, if they were to be used, they would have the same model with $v_{D G 3}=v_{D G}=v_{G}$.

Figure 3-5 shows the islanded condition. The microgrid will solely supply the overall load demand. Therefore, the VCM should be adopted by the DG inverter(s) to regulate the PCC voltage with a desired magnitude and frequency generated by the droop controller. The state-space model for DG inverters of the SB and the PEMFC stack can be written as follows:

$$
\dot{x}_{i}=A_{i} x_{i}+B_{i 1} i^{\prime}+B_{i 2} u
$$

where the subscript $i$ denotes islanded condition and

$$
A_{i}=\left[\begin{array}{ccc}
-\frac{R_{3}}{L_{f 3}} & 0 & -\frac{1}{L_{f 3}} \\
0 & -\frac{R_{4}}{L_{f 4}} & \frac{1}{L_{f 4}} \\
\frac{1}{C_{f}^{\prime}} & \frac{1}{C_{f}^{\prime}} & 0
\end{array}\right] ; B_{i 1}=\left[\begin{array}{c}
0 \\
0 \\
-\frac{1}{C_{f}^{\prime}}
\end{array}\right] ; B_{i 2}=\left[\begin{array}{cc}
\frac{V_{d c 3}}{L_{f 3}} & 0 \\
0 & \frac{V_{d c 4}}{L_{f 4}} \\
0 & 0
\end{array}\right]
$$


with $C_{f}^{\prime}=C_{f 3}+C_{f 4} ; x_{i}=\left[\begin{array}{lll}i_{3} & i_{4} & v_{G}\end{array}\right]^{T} ; i^{\prime}=i_{D G 3}+i_{D G 4} ; u=\left[\begin{array}{ll}u_{3} & u_{4}\end{array}\right]^{T}$. Since $i_{D G 3}+i_{D G 4}=i_{G 1}+i_{G 2}$ and $i_{G j}=i_{L j}-i_{D G j}(j=1,2)$, it follows from (3.7) and (3.8) that, neglecting the line impedances within DG inverters 1 and 2 (hence $v_{D G j}=v_{G}$ ), a unified model can be obtained for all DG inverters during islanded conditions:

$$
\begin{gathered}
\dot{x}_{i j}=A_{i j} x_{i j}+B_{i j 1} i_{j}^{\prime}+B_{i j 2} u_{j} \\
y_{i j}=C_{i j} x_{i j}+D_{i j 1} i_{j}^{\prime}+D_{i j 2} u_{j}
\end{gathered}
$$

where $j=1,2,3,4$ and

$$
A_{i j}=\left[\begin{array}{cc}
-\frac{R_{j}}{L_{f j}} & -\frac{1}{L_{f j}} \\
\frac{1}{C_{f}^{\prime}} & 0
\end{array}\right] ; B_{i j 1}=\left[\begin{array}{c}
0 \\
-\frac{1}{C_{f}^{\prime}}
\end{array}\right] ; B_{i j 2}=\left[\begin{array}{c}
\frac{V_{d c j}}{L_{f j}} \\
0
\end{array}\right] ; C_{i j}=\left[\begin{array}{cc}
0 & 1 \\
1-\frac{C_{f j}}{C_{f}^{\prime}} & 0
\end{array}\right] ; D_{i j 1}=\left[\begin{array}{c}
0 \\
\frac{C_{f j}}{C_{f}^{\prime}}
\end{array}\right] ;
$$

$D_{g j 2}=\left[\begin{array}{l}0 \\ 0\end{array}\right] ; \quad$ with $\quad C_{f}^{\prime}=\sum_{n=1}^{4} C_{f n} \quad ; \quad x_{i j}=\left[\begin{array}{ll}i_{j} & v_{D G j}\end{array}\right]^{T} \quad$ is the state vector; $i_{j}^{\prime}=\sum_{m} i_{L m}-\sum_{n \neq j} i_{n}$ is the exogenous input of the DG inverter $j ; u_{j}$ is the control input, with $-1 \leq u_{j} \leq 1$; and $y_{i j}=\left[\begin{array}{ll}v_{D G j} & i_{D G j}\end{array}\right]^{T}$ is the output, which will be regulated according to references. Even though it is called VCM, both $v_{D G j}$ and $i_{D G j}$ will be regulated to guarantee the power delivered. In addition, the exogenous input $i_{j}$ cannot be measured directly because it includes quantities outside the inverter, as shown above. However, $i_{j}{ }_{j}$ can be obtained by applying a Kalman filter to the output $y_{i j}$. It will be detailed in Section 3.3.

\subsubsection{Droop Control for Parallel Operation of Inverters}

A fault occurs in the upstream network which results in the opening of the $\mathrm{CB}$, which isolates the microgrid from the grid. Consequently, the only power sources that can supply the loads are the DG units shown in Figure 3-1. The real and reactive power demand $\Delta P$ and $\Delta Q$ will be shared among $n$ DG inverters as follows: 


$$
\begin{gathered}
\Delta P=\sum_{j=1}^{n} \Delta P_{j} \\
\Delta Q=\sum_{j=1}^{n} \Delta Q_{j}
\end{gathered}
$$

where $\Delta P_{j}$ and $\Delta Q_{j}$ are the real and reactive power variations of the $j$ th DG inverter, respectively

The droop control method proposed in [66], [117] is used for the sharing of the real and reactive power within the microgrid. It regulates the magnitude and frequency of $v_{D G j}$ as the $j$ th DG inverter is operating in VCM:

$$
\begin{gathered}
\omega_{j}=\omega_{r e f}-k_{P, j} \times\left(P_{r e f, j}-P_{j}\right) \\
v_{D G j}=v_{r e f}-k_{Q, j} \times\left(Q_{r e f, j}-Q_{j}\right)
\end{gathered}
$$

where $P_{j}$ and $Q_{j}$ represent the actual active and reactive power outputs of the $j$ th DG unit; $k_{P, j}$ and $k_{Q, j}$ are the droop characteristics of the $j$ th DG unit; $\omega_{j}$ and $v_{D G j}$ are the frequency and magnitude of the output voltage of the $j$ th DG unit; $P_{r e f, j}$ and $Q_{r e f, j}$ are the real and reactive power references based on power rating of the DG unit; and $\omega_{\text {ref }}$ and $v_{r e f}$ are the references for the frequency and magnitude of the output voltage during islanded condition.

\subsection{Model Predictive Control Design of DG Inverters}

Some research works can be found that focus on the application of MPC for inverter control. In [93], the authors present a Finite Control Set Model Predictive Control scheme. This scheme can achieve successful control over different converters without additional modulation techniques or internal cascade control loops. However, the authors do not consider the parallel operation of the inverters. In [118], the feasibility of MPC in controlling parallel-connected inverters are investigated. Nonetheless, the authors focus mainly on the application of the inverters in uninterruptible power supplies that are in standalone operation. Nonetheless, limited research works can be 
found on the implementation of MPC for parallel operation of DG inverters in microgrids.

A newly developed MPC algorithm for paralleled DG inverters is proposed in this research using the mathematical model developed in Section 3.2. This MPC algorithm decomposes the MPC optimization into two subproblems: a steady-state subproblem and a transient subproblem. A moving horizon method is adopted to solve both problems in grid-connected and islanded conditions of the proposed microgrid. This approach can greatly reduce the computational time, and therefore, it is designed for fast sampling systems to regulate periodic signals.

Whether the microgrid operates in grid-connected or islanded condition, the discretized state-space model of the DG inverters can be written as follows:

$$
\begin{gathered}
x^{+}=A x+B_{1} w+B_{2} u \\
y=C x+D_{1} w+D_{2} u
\end{gathered}
$$

where the superscript + represents the time-shift operator (the sampling interval is set to be $T_{s}$ ), and $\mathrm{w}$ is the periodic exogenous signal. As understood, for any periodic signal that has a finite number of harmonics, this signal can be further expressed as an autonomous finite dimensional LTI state-space model. For instance, for a periodic signal whose fundamental frequency is $\omega$ and that consists of only odd harmonics, the $A$-matrix of the corresponding state-space model then can be written as $\left[\begin{array}{cc}\cos \left(h \omega T_{s}\right) & \sin \left(h \omega T_{s}\right) \\ -\sin \left(h \omega T_{s}\right) & \cos \left(h \omega T_{s}\right)\end{array}\right]$ where $\quad h=1, \quad 3, \quad 5, \quad \ldots, \quad$ and the $C$-matrix is $\left[\begin{array}{lllllll}1 & 0 & 1 & 0 & \ldots & 1 & 0\end{array}\right]$. Additionally, the initial state of this autonomous model can determine the magnitude and phase angle of this periodic signal. Therefore, the exogenous signal $w$ in (3.17) and (3.18) and the reference $\mathrm{d}$ can be presented as:

$$
\begin{gathered}
\xi^{+}=A_{\xi} \xi \\
w=C_{w} \xi \\
d=C_{d} \xi
\end{gathered}
$$


for some $A_{\xi}, C_{w}$ and $C_{d}$ as described above. When in grid-connected condition, the DG inverter of the MT or the PV array is operating in CCM. The reference $d$, which $i_{D G}$ is required to track, and the output $y$ typically should consist of the same order of harmonics as $i_{L}$ (or some harmonics of $i_{L}$ will contaminate the distribution grid). Additionally, this order of harmonics is derived from the required real and reactive power outputs of the DG unit. However, the DG inverters of the SB and the PEMFC stack operate in VCM during islanded condition. Furthermore, $y=\left[v_{D G} i_{D G}\right]^{\mathrm{T}}$ for the DG units and the first part of the reference $d$, which is tracked by $v_{D G}$, is typically a sinusoidal signal. The frequency and magnitude of this waveform are determined by (3.13) and (3.14), respectively.

The exogenous system is shown in the state-space model in (3.19) - (3.21). This research only considers odd harmonics up to the 29th order. However, this proposed method can be used to consider even harmonics or high-order harmonics. The sets of the Fourier coefficients of $w$ and $d$ are indicated as the exogenous state $\xi$. This exogenous state can be identified through a Kalman filter based observer. During islanded condition, although $w$ cannot be measured, it can be obtained through the observer by combining the exogenous system with the DG inverter model in (3.11) and (3.12) using the measurement of $y$ and the known $d$.

The simulation studies in this research use a sampling interval of $T_{s}=0.2 \mathrm{~ms}$. This sampling interval selected is actually considered very fast in terms of traditional MPC application. Yet it is necessary for such a problem that intends to deal with high-order harmonics. According to [119], such a sampling in the range of tens of $\mathrm{kHz}$ is possible with state-of-the-art technology.

The control signal $u$ in (3.17) and (3.18) is divided into two components:

$$
u=u_{s}+u_{t}
$$

where $u_{s}$ is the steady-state control signal, and $u_{t}$ is the transient control signal. It is assumed that $u \rightarrow u_{s}$ and $u_{t} \rightarrow 0$ asymptotically. These two components both will be 
considered in the MPC algorithm. The steady-state part will adopt a dynamic MPC algorithm, while the transient part will adopt a more conventional finite-horizon approach. This is because the conventional finite-horizon approach is relatively more computationally intensive. The steady-state part will be calculated through a different algorithm that uses proper trade-offs for best possible performance for a low complexity. By doing so, a smaller $T_{s}$ then can be chosen for such a fast sampling system.

\subsubsection{Steady-State Subproblem}

In the steady-state subproblem, $x \rightarrow x_{s}, y \rightarrow y_{s}$ and $u \rightarrow u_{s}$ asymptotically. According to (3.17) and (3.18), the following equations can be obtained:

$$
\begin{aligned}
& x_{s}^{+}=A x_{s}+B_{1} w+B_{2} u_{s} \\
& y_{s}=C x_{s}+D_{1} w+D_{2} u_{s}
\end{aligned}
$$

subject to

$$
\left|u_{s}\right| \leq 1
$$

Ideally, $y_{s}$ should equal the reference $d$; i.e., defining a tracking error as follows:

$$
e_{s}=y_{s}-d=0
$$

However, this ideal situation cannot always be realized because of the constraint shown in (3.25). A dynamic MPC algorithm is used to generate the steady-state control $u_{s}$ as follows:

$$
\begin{aligned}
& \hat{\xi}^{+}=A_{\hat{\xi}} \hat{\xi} \\
& u_{s}=C_{\hat{\xi}} \hat{\xi}
\end{aligned}
$$


where the matrices $A_{\hat{\xi}}$ and $C_{\hat{\xi}}$ are defined offline, but the initial state $\hat{\xi}$ at time $k$ will be calculated online using a moving horizon. Therefore, $x_{s}$ can be expressed as:

$$
x_{s}=X \xi+\hat{X} \hat{\xi}
$$

where the matrices $X$ and $\hat{X}$ satisfy the following:

$$
\begin{aligned}
& X A_{\xi}=A X+B_{1} C_{w} \\
& \hat{X} A_{\xi}=A \hat{X}+B_{2} C_{\hat{\xi}}
\end{aligned}
$$

Additionally, both $y_{s}$ and $e_{s}$ are linear functions of $\xi$ and $\hat{\xi}$ as follows:

$$
\begin{aligned}
y_{s} & =C x_{s}+D_{1} w+D_{2} u_{s} \\
& =\left(C X+D_{1} C_{w}\right) \xi+\left(C \hat{X}+D_{2} C_{\hat{\xi}}\right) \hat{\xi} \\
e_{s} & =y_{s}-d \\
& =y_{s}-C_{d} \xi \\
& =\left(C X+D_{1} C_{w}-C_{d}\right) \xi+\left(C \hat{X}+D_{2} C_{\hat{\xi}}\right) \hat{\xi}
\end{aligned}
$$

The exogenous state $\xi$ is estimated online by the Kalman-based observer. Therefore, the follow optimization problem

$$
\begin{gathered}
\min _{\hat{\xi}(k)} \sum_{i} e_{s}(k+i)^{T} Q e_{s}(k+i) \\
\text { subject to }\left|u_{s}(k+i)\right| \leq 1
\end{gathered}
$$

then can be solved by obtaining the optimal control state $\hat{\xi}(k)$ and, accordingly, the steady-state control $u_{s}(k)$, where the summation in (3.34) is for a period of $0.02 \mathrm{~s}$, given that the $Q$ is a given positive definite matrix. The model presented in (3.27) and (3.28) is a prediction model for future $u_{s}$, while executive $u_{s}$ will be recomputed from (3.34) 
with a moving horizon. The matrices $A_{\hat{\xi}}$ and $C_{\hat{\xi}}$ only can be determined under the conditions below:

1) Systems (3.27) and (3.28) are observable.

2) $A_{\hat{\xi}}$ has the same order of harmonics as $A_{\xi}$ in (3.19).

3) A transformation matrix $T$ exists, such that $\hat{\xi}=T \xi$ for all time and it always results in $e_{s}=0$; i.e., the optimization

$$
\min _{\hat{\xi}(k)} \sum_{i} e_{s}(k+i)^{T} Q e_{s}(k+i)
$$

will have value zero attained at $\hat{\xi}(k)=T \xi(k)$.

A unique optimizer $\hat{\xi}(k)$ will be guaranteed with the above conditions. In addition, when the constraint $\left|u_{s}\right| \leq 1$ is not violated, the dynamic control laws stated in (3.27) and (3.28) are equivalent to the optimal state-feedback law given by $u_{s}=C_{\hat{\xi}} T \xi$.

\subsubsection{Transient Subproblem}

The transient signals $u_{t}=u-u_{s}, x_{t}=x-x_{s}$ and $y_{t}=y-y_{s}$ are defined to solve the transient subproblem. According to (3.17), (3.18), (3.23) and (3.24), $u_{t}, x_{t}$, and $y_{t}$ should satisfy the following:

$$
\begin{gathered}
x_{t}^{+}=A x_{t}+B_{2} u_{t} \\
y_{t}=C x_{t}+D_{2} u_{t}
\end{gathered}
$$

The objective is to make $y_{t} \rightarrow 0$ as soon as possible, subject to

$$
\left|u_{s}+u_{t}\right| \leq 1
$$

Such a transient problem can be solved by a conventional MPC algorithm that adopts a finite horizon with a terminal cost. Specifically, for the given positive definite matrices $Q$ and $R$ and the chosen length of control horizon $N_{u}$, the following optimization should be solved: 


$$
\begin{aligned}
& \min _{u(k+i)} \sum_{i=1}^{N_{u}}\left(y_{t}(k+i)^{T} Q y_{t}(k+i)+u_{t}(k+i)^{T} R u_{t}(k+i)\right) \\
& \quad+x_{t}\left(N_{u}+1\right)^{T} P_{T} x_{t}\left(N_{u}+1\right) \\
& \quad \text { subject to }\left|u_{s}(k+i)+u_{t}(k+i)\right| \leq 1
\end{aligned}
$$

The matrix $A$ in the present case is stable. Therefore, an appropriate selection of $P_{T}$ is the weighted observability Gramian obtained from the following Lyapunov equation:

$$
A^{T} P_{T} A-P_{T}+C^{T} Q C=0
$$

This makes the optimization in (3.39) equivalent to the following optimization:

$$
\begin{gathered}
\min _{u(k+i)} \sum_{i=1}^{\infty}\left(y_{t}(k+i)^{T} Q y_{t}(k+i)+u_{t}(k+i)^{T} R u_{t}(k+i)\right) \\
\text { subject to }\left|u_{s}(k+i)+u_{t}(k+i)\right| \leq 1 \text { for } i \leq N_{u} \\
\text { and } u_{t}(k+i)=0 \text { for } i>N_{u}
\end{gathered}
$$

It can be seen from (3.41) that this optimization requires $u_{s}$ and $x_{s}$. These two can be obtained through the steady-state subproblem. It also requires the information of the system state $x$. This can be estimated using a Kalman-based observer on (3.17) and (3.18). Figure 3-7 shows the overall configuration of the proposed control algorithm, which includes the steady-state control signal and the transient control signal. The control and prediction horizons of the transient MPC are both set to 10 with a carefully determined terminal cost. The steady-state MPC does not have a control horizon as it is a dynamic MPC algorithm. The prediction horizon is set to 50 which corresponds to half of the fundamental period of $50 \mathrm{~Hz}(50 * 0.2 \mathrm{~ms}=0.01 \mathrm{~s})$. Note that the objective of the steady-state MPC is to provide the reference signals for voltage and current, and it is possible to update at a lower frequency if required. 


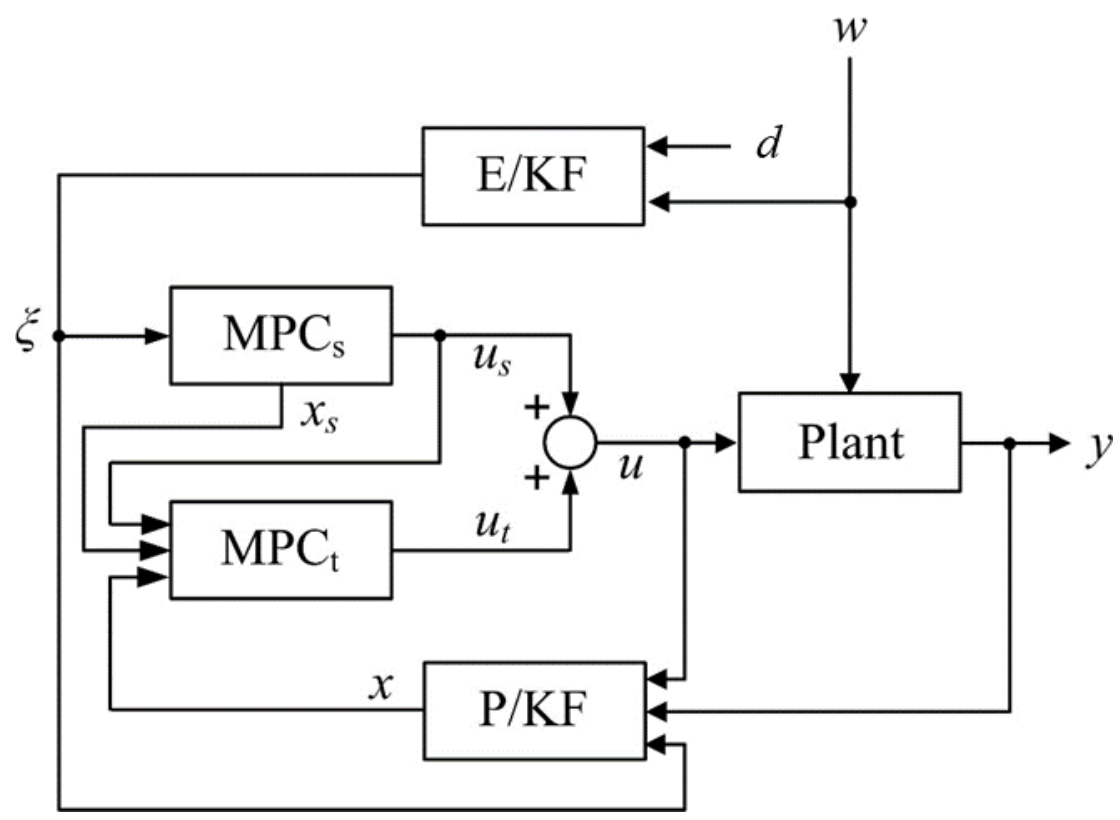

Figure 3-7: Overall MPC controller for the DG inverter with E/KF denoting the exogenous Kalman filter and P/KF denoting the plant Kalman filter.

\subsection{Simulation Studies}

Matlab/Simulink is used for the simulation studies of this chapter. Different scenarios in both grid-connected and islanded conditions are tested in simulation environment. Both linear load and nonlinear load are considered in the simulation studies. A threephase dimmer load is introduced as Load 1; it has real power demand of $P_{L, l}=15 \mathrm{~kW}$ and reactive power demand of $Q_{L, 1}=9.7 \mathrm{kVAr}$. In the Matlab/Simulink environment, a current source is used to simulate this dimmer load using the experimental results of different light dimmers described in [120]. A three-phase RL load is introduced as Load 2; it has real power demand of $P_{L, 2}=5 \mathrm{~kW}$ and reactive power demand of $Q_{L, 2}=3$ kVAr. Table 3-1 includes the system parameters in the simulated system. The detailed parameters of the distribution lines and the transformer are obtained using the data in [121]. Rough estimation has been made for DG internal resistance, as it is generally unknown. 
Table 3-1: Parameters of the Proposed System

\begin{tabular}{ll}
\hline \hline \multicolumn{1}{c}{ Parameter } & \multicolumn{1}{c}{ Value } \\
\hline Distribution grid voltage & $v_{G}=230 \mathrm{Vrms}$ (phase) \\
DC-link voltage & $V_{d c}=800 \mathrm{Vdc}$ \\
Distribution line impedance & $R_{\ell}=7.5 \mathrm{~m} \Omega, L_{\ell}=25.7 \mu \mathrm{H}$ \\
Transformer impedance & $R_{T}=1.9 \mathrm{~m} \Omega, L_{T}=28.6 \mu \mathrm{H}$ \\
LC filter & $L_{f}=1.2 \mathrm{mH}, C_{f}=20 \mu \mathrm{F}$ \\
DG inverter loss resistance & $R_{f}=0.01 \Omega$ \\
\hline \hline
\end{tabular}

\subsubsection{Test Case 1: Power Quality Improvement with Load Sharing During Grid-Connected Condition}

To reduce the power delivered from the upstream network and the generation burden, the main DG units in the microgrid are required to supply a certain amount of power to the loads during grid-connected condition. This scenario is demonstrated in Test Case 1 with the power flow managed among the upstream distribution network, the DG inverters and the loads. DG inverter 1, which is the MT, is required to supply $50 \%$ of $P_{L, 1}$, while DG inverter 2 , which is the PV, is required to supply $60 \%$ of $P_{L, 2}$. The remaining real power load demand is supplied by the distribution grid. As mentioned in Section 3.2, the SB is charged during the off-peak period when the cost of power generation from the distribution grid is low. The PEMFC stack is in idle mode in case of a sudden demand for power. Test Case 1 is also conducted to demonstrate that the DG units are able to compensate for the harmonics in the load currents, and improve the power quality of the distribution network. For this purpose, DG inverter 1, which is shown in Figure 3-1, is required to compensate for the harmonics in the load current of Load 1, as it contains a nonlinear three-phase dimmer load. By doing so, the harmonics will be compensated locally and will not cause any harmonic problem for the microgrid and the upstream network.

Figure 3-8 shows the total load current absorbed by Load $1 i_{L 1}$, the current supplied by DG inverter 1 to Load $1 i_{D G l}$, and the current supplied by the grid to Load $1 i_{G l}$ for $0<t<0.2 \mathrm{~s}$. It can be seen that during the initialization period of $0<t<0.06 \mathrm{~s}, i_{G 1}$ is gradually stabilized to steady-state because the DG inverter controller needs 
approximately three cycles to follow the reference steadily. In this period, a large transient inrush current is observed in $i_{D G 1}$, as shown in Figure 3-8. In this research, it is assumed that the DG inverter can withstand such a large transient current. The total harmonic distortion (THD) of $i_{L 1}$ in steady-state is $41.7 \%$. With the implementation of the proposed MPC control to regulate the output current of DG inverter 1, the THD of $i_{G l}$ is now improved to approximately $0.4 \%$, as shown in Figure 3-8.
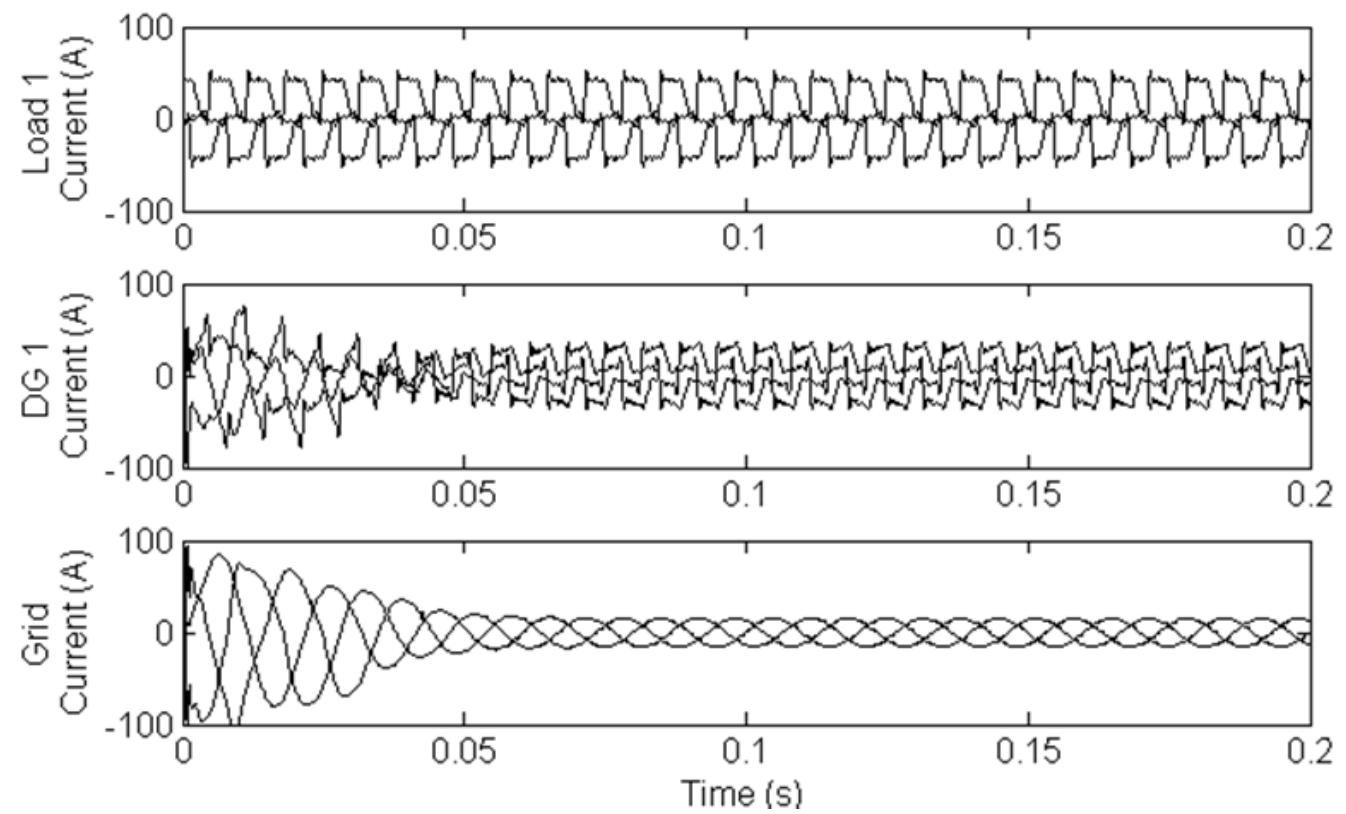

Figure 3-8: Waveforms of three-phase Load 1 current $i_{L 1}$ (top), three-phase DG 1 current $i_{D G l}$ (middle), and three-phase grid current $i_{G l}$ (bottom).

In addition, to guarantee a unity power factor at the distribution grid side, the DG inverter 1 will be controlled to deliver the entire reactive component $i_{L f l, q}$ of $i_{L 1}$ as in (3.5). The result can be seen in Figure 3-9, which shows the zoom-in waveforms of the grid voltage $v_{G}$ (the voltage has been scaled down by a factor of 0.25 for comparison purposes) and $i_{G 1}$ for $0<t<0.14 \mathrm{~s}$. It can be seen that $i_{G 1}$ is in phase with that of $v_{G}$ with this power factor correction function. 


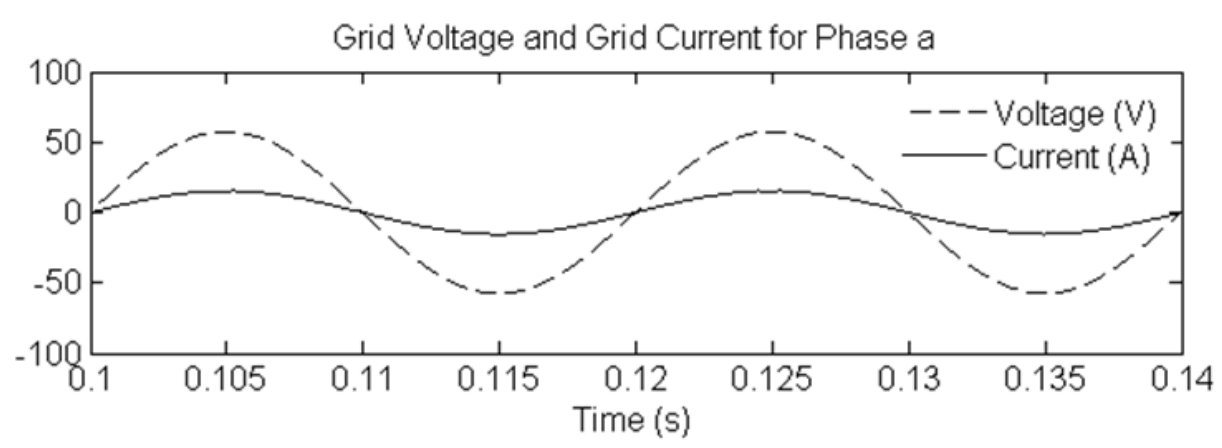

Figure 3-9: Waveforms of grid voltage $v_{G}$ and grid current $i_{G 1}$ for phase a.

The real and reactive power delivered by DG inverter 1 and 2 for $0<t<0.2 \mathrm{~s}$ are shown in Figures 3-10 and 3-11, respectively. For real power balance, DG inverter 1 and 2 supply $7.5 \mathrm{~kW}\left(50 \%\right.$ of $\left.P_{L, 1}\right)$ and $3 \mathrm{~kW}\left(60 \%\right.$ of $\left.P_{L, 2}\right)$ respectively. This proves that the proposed MPC algorithm can track and control the inverters to deliver the required real power. Similarly, for reactive power compensation, DG inverter 1 and 2 are required to inject all the reactive power of $12.7 \mathrm{kVAr}$ to be absorbed by the loads to maintain unity power factor at the grid side. Figure 3-12 shows the real and reactive power delivered by the upstream distribution network to Loads 1 and 2, and to charge the SB for $0<t<0.2 \mathrm{~s}$. It can be seen that the upstream distribution network delivers 7.5 $\mathrm{kW}\left(50 \%\right.$ of $\left.P_{L, 1}\right)$ to Load $1,2 \mathrm{~kW}\left(40 \%\right.$ of $\left.P_{L, 2}\right)$ to Load 2, and an additional power of $1.5 \mathrm{~kW}$ to the SB. Additionally, it can be seen that as DG inverter 1 and 2 have compensated for all the reactive power required by the loads, the reactive power supplied by the distribution grid is zero, which ensures unity power factor at the grid side. 

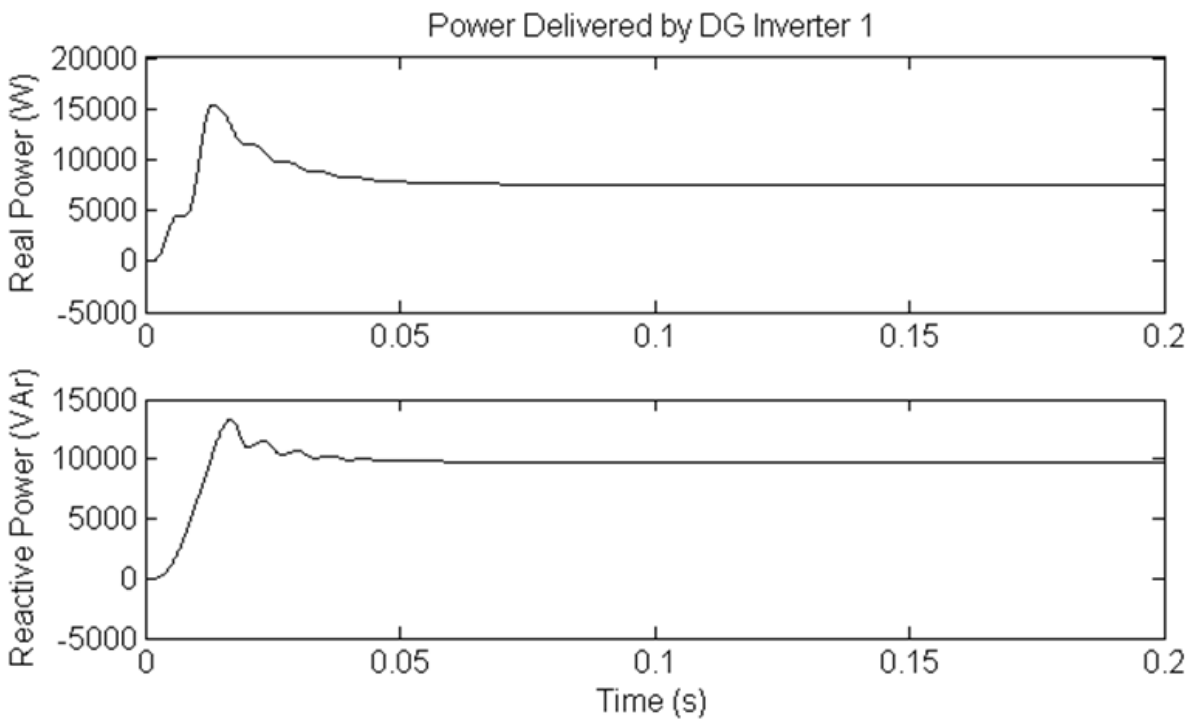

Figure 3-10: Real (top) and reactive (bottom) power delivered by DG inverter 1.
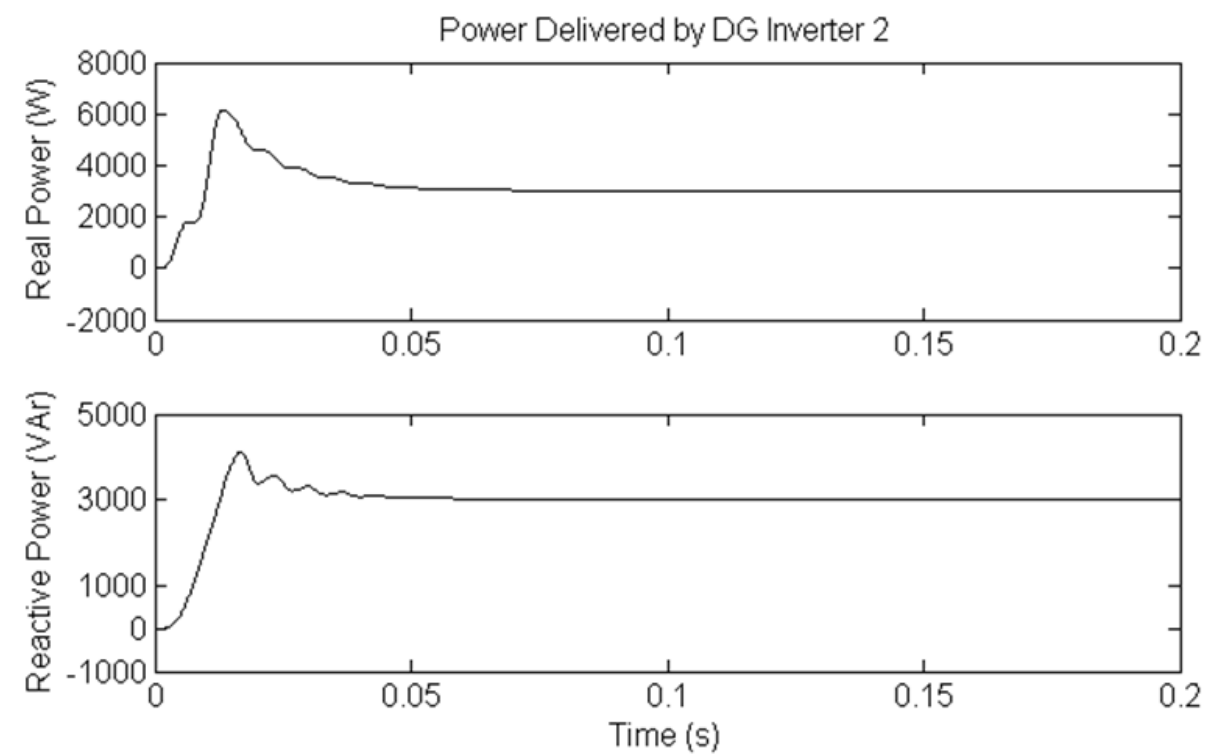

Figure 3-11: Real (top) and reactive (bottom) power delivered by DG inverter 2. 

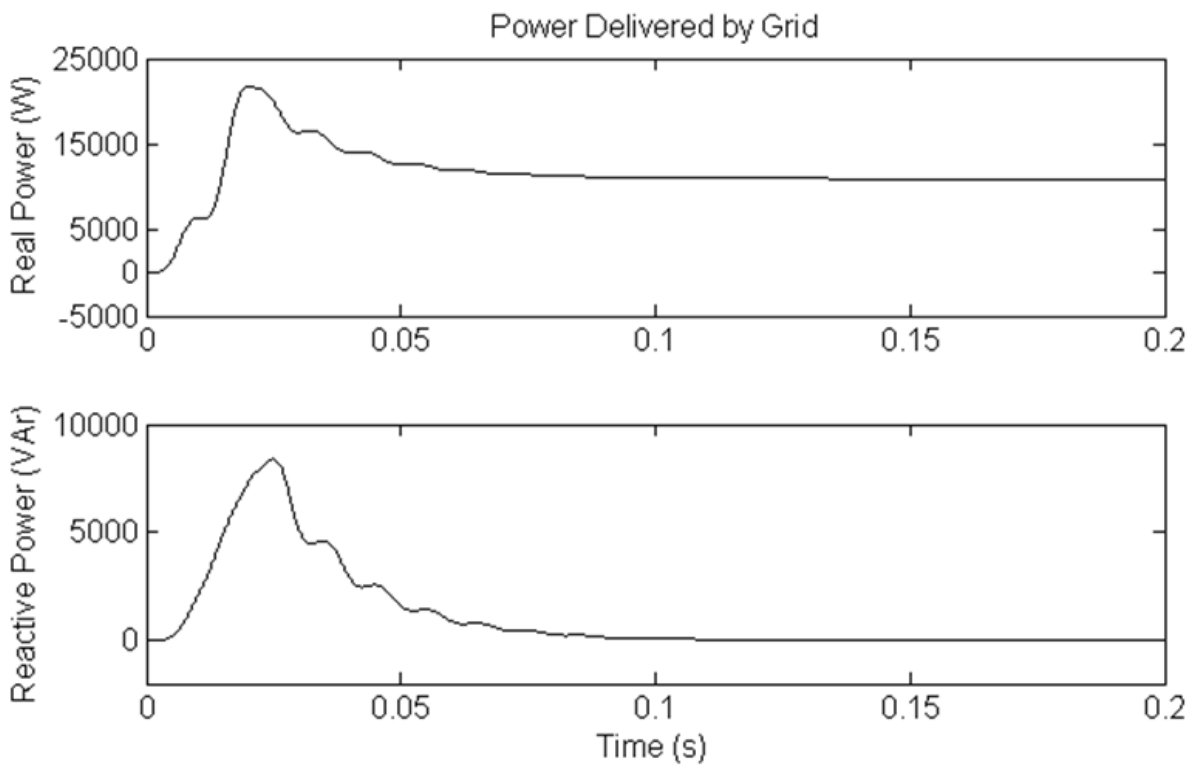

Figure 3-12: Total real (top) and reactive (bottom) power delivered by the grid to Loads 1 and 2, and to charge the SB.

\subsubsection{Test Case 2: Load Sharing during Islanded condition with Droop Control}

In Test Case 2, the backup DG units (i.e., the SB and the PEMFC stack) are activated when the microgrid switches to islanded condition. It demonstrates that the backup DG units can share the real and reactive power when the operating condition changes. For $0<t<0.2 \mathrm{~s}$, the microgrid stays in grid-connected condition. At $t=0.2 \mathrm{~s}$, a fault occurs in the upstream network of the distribution grid, and the CB, which is shown in Figure 31 , is opened to cut the connection between the microgrid and the upstream network.

As shown in Figure 3-13 which is the real and reactive power delivered by DG inverter 1 for $0.1<t<0.4 \mathrm{~s}$, the MT increases its real power generation from $7.5 \mathrm{~kW}$ to approximately $11.4 \mathrm{~kW}$ during $0.2<t<0.4 \mathrm{~s}$. The reactive power generated by the MT remains at $9.7 \mathrm{kVAr}$ which is the same in Test Case 1 . The real and reactive power delivered by DG inverter 2 for $0.1<t<0.4 \mathrm{~s}$ is shown in Figure 3-14. It can be seen that the PV maintains its real and reactive power generations at $3 \mathrm{~kW}$ and $3 \mathrm{kVAr}$ throughout this period. This is because the PV is constantly working in MPPT mode. From the above-mentioned analysis, it can be observed that the real power generation from the MT and the PV cannot meet the total real power load demand. Hence, the SB and PEMFC stacks are controlled to deliver the required real power to the load. This 
can be achieved through the frequency and voltage droop control method through a centralized approach by sending the frequency and voltage references to all DG units. As the MT and the PV are compensating for all the reactive power required by the load, the backup DG units are not required to provide reactive power sharing. This can avoid inaccurate reactive power sharing due to network cable impedances [66], [117], [122], and also reduce the transmission line losses.
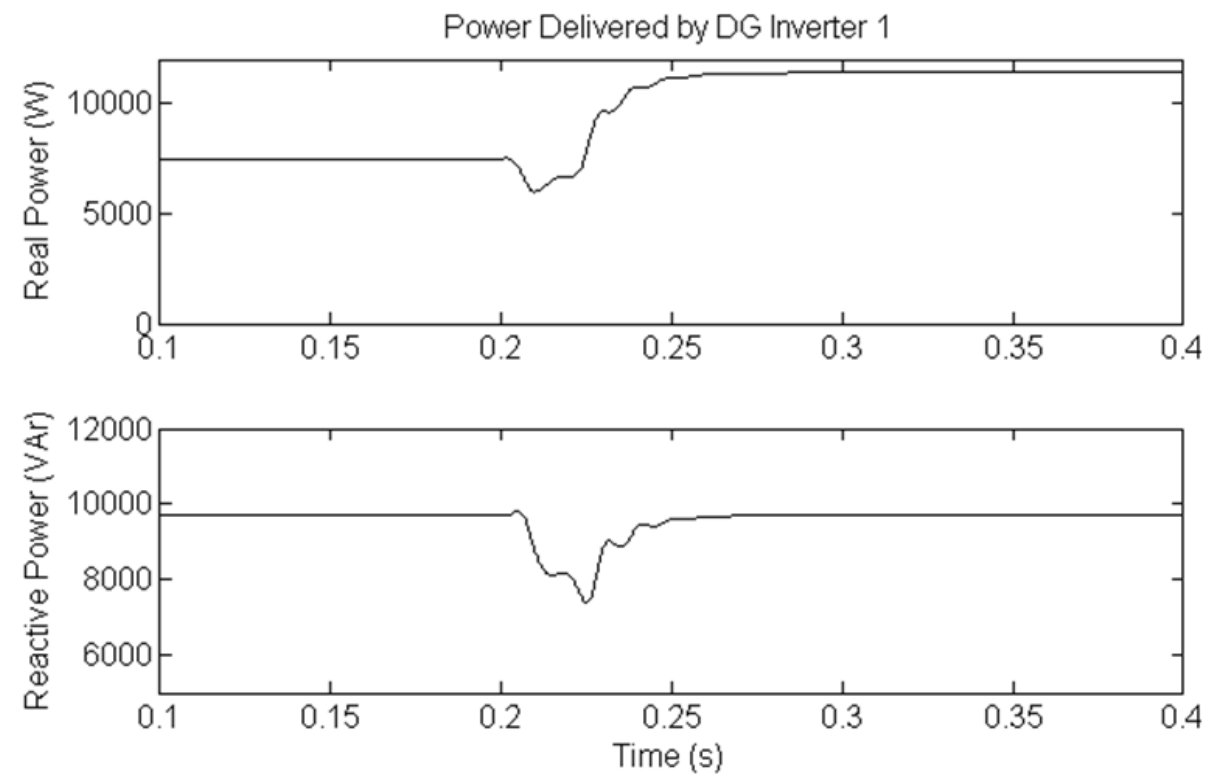

Figure 3-13: Real (top) and reactive (bottom) power delivered by DG inverter 1.
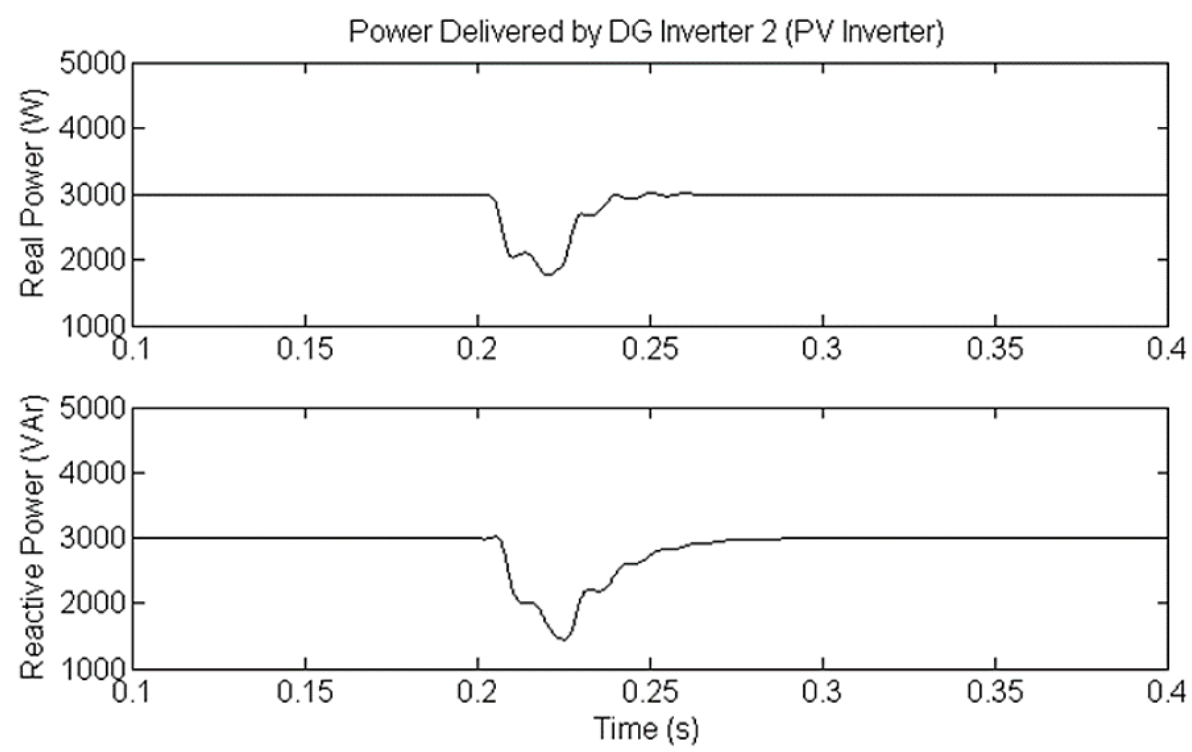

Figure 3-14: Real (top) and reactive (bottom) power delivered by DG inverter 2. 
Figures 3-15 and 3-16 show the real power delivered by DG inverter 3 and 4, respectively, for $0.1<t<0.4 \mathrm{~s}$. For $0<t<0.2 \mathrm{~s}$, both the SB and the PEMFC stacks are in idle mode. After the microgrid transitions into islanded condition at $t=0.2 \mathrm{~s}$, DG inverter 3 and 4 increase their real power generation to approximately $3.28 \mathrm{~kW}$ and $2.28 \mathrm{~kW}$ respectively. It can be seen from both waveforms that the proposed MPC algorithm can make the DG inverters track and achieve steady-state operation in about 0.5 cycle. Figure 3-17 shows the waveform of $v_{D G}$, in which it can be observed that the frequency of $v_{D G}$ drops from its nominal value of $50 \mathrm{~Hz}$ to $49.8 \mathrm{~Hz}$ to realize the frequency droop control for real power sharing.

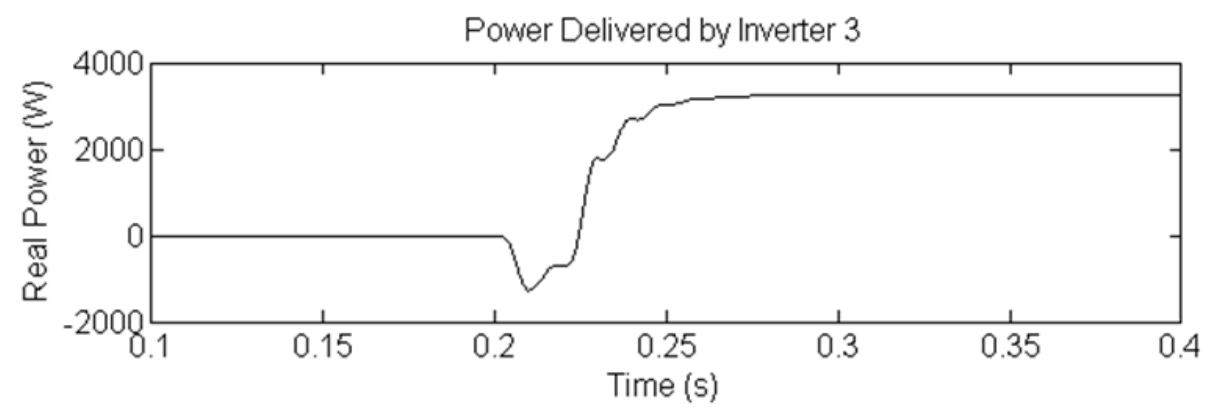

Figure 3-15: Real power delivered by DG inverter 3.

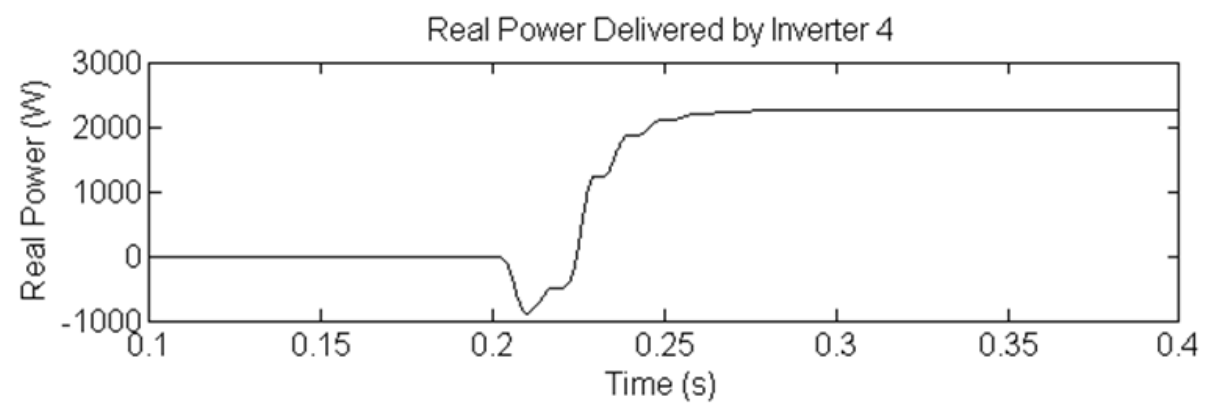

Figure 3-16: Real power delivered by DG inverter 4 .

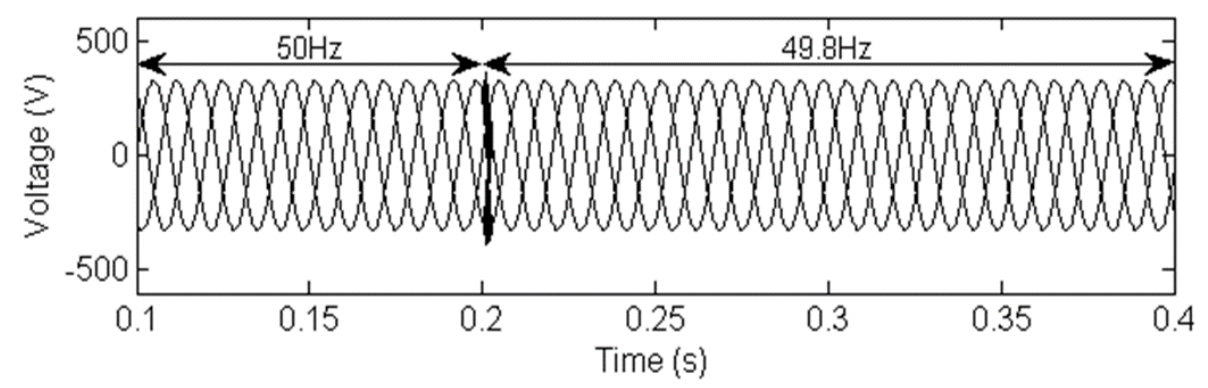

Figure 3-17: Inverter output voltage $v_{D G}$ of DG units 3 and 4. 


\subsubsection{Test Case 3: Investigation on Network Voltage Changes}

In Singapore's low-voltage distribution network, operators are required to maintain the voltage of the distribution network within $\pm 6 \%$ of its nominal value. In the proposed microgrid configuration, which is shown in Figure 3-1, all the DG units are connected to the PCC directly through the distribution line. Therefore, during islanded condition, the change of the real and reactive power flows will affect the voltage profiles for the loads that are connected to the microgrid at the PCC. The voltage change $\Delta V$ can be formulated as follows:

$$
\Delta V=\frac{P R+Q X}{V}
$$

where $R$ and $X$ are the resistance and inductance of the distribution line, and $V$ is the voltage at the load side. It can be concluded from (3.42) that the voltage at the load side can be greatly affected by both the resistance and inductance of the distribution line [123].

In Test Case 3, the voltage at PCC is investigated when different load demands are connected to the PCC during islanded condition. Specifically, instead of Load 1 and Load 2, an initial load of $2 \mathrm{~kW}$ and $1.24 \mathrm{kVAr}$ is connected to the microgrid at the PCC as shown in Figure 3-1. To analyze the effect of the real and reactive power demand on the PCC voltage, a step size of $1 \mathrm{~kW}$ and $0.62 \mathrm{kVAr}$ of load is added to the PCC every $0.1 \mathrm{~s}$ for $0<t<1 \mathrm{~s}$. The choices of real and reactive power demand step to be $1 \mathrm{~kW}$ and $0.62 \mathrm{kVAr}$, respectively, are to maintain the power factor at approximately 0.85 .

The result is shown in Figure 3-18. It can be observed from this figure that each increment causes a significant voltage drop in per unit (p.u.) value in the voltage at PCC as explained by (3.42). The voltage has dropped from about 0.96 p.u. at the initial condition to about 0.75 p.u. Therefore, the conclusion can be made that in islanded condition, the increases of the real and reactive power demands will increase the voltage drop to at the PCC. To solve this problem, different solutions can be used. For 
instance, a traditional technique is to use a tap-changing transformer that can control the voltage at the PCC [124].

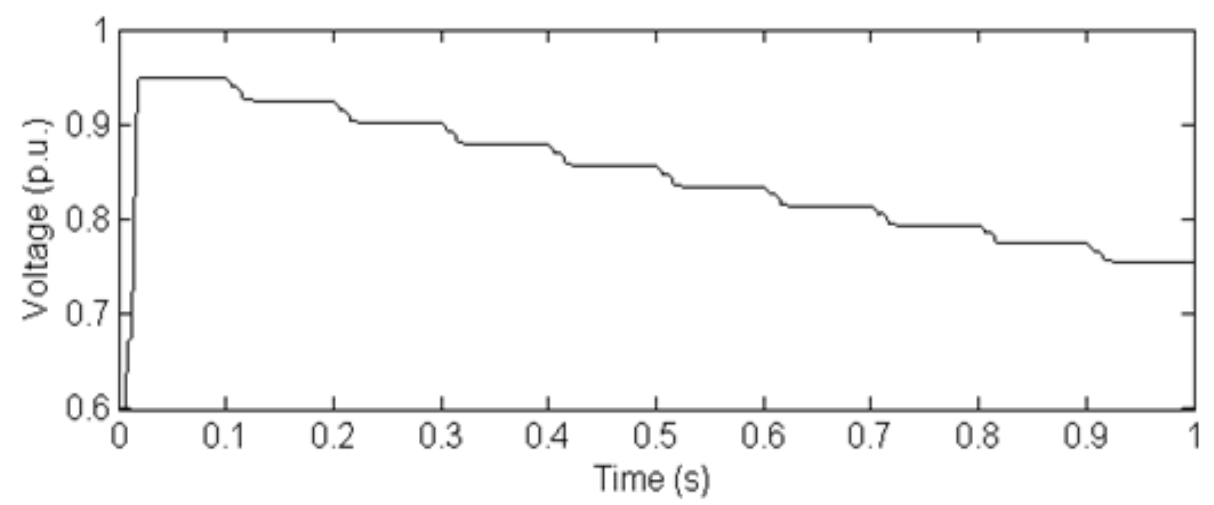

Figure 3-18: Voltage profile (in p.u.) for changes in the real and reactive power of the load demand.

\subsubsection{Test Case 4: Investigation on Increased Fault Level}

As the DG units are connected to the microgrid through DG inverters, the fault level of the low-voltage distribution network will be altered. A more complicated flow of fault currents will occur during any fault happening in the microgrid. Test Case 4 investigates the fault level based on the proposed microgrid configuration. The results can affect the operations of the CB in Figure 3-1 and the distribution lines.

Test Case 4 features a scenario in which a fault occurs on the distribution line that connects the microgrid and the MT (Point F), as shown in Figure 3-1 in gridconnected condition. Figure 3-19 is the equivalent single-line diagram of the microgrid shown in Figure 3-1 to illustrate the situation in this test. It can be seen that fault currents will be drawn from the PV, the MT, and the distribution network. Different values of currents and voltages at different points of the distribution network during the fault at Point $F$ can be found in Table 3-2. The fault currents from DG inverter 1 and 2, and the grid for $0<t<0.1 \mathrm{~s}$ are shown in Figures 3-20, 3-21, and 3-22, respectively. According to relevant literature [117], [124], the DG inverter can only supply a certain amount of short-circuit current typically from 1.5 p.u. to 2 p.u. Therefore, in this test case, the fault currents drawn from the DG inverters of the MT and the PV are restricted to 2 p.u. It then can be concluded that the integration of the 
MT and the PV in the distribution network will increase the fault level at point $F$ by about $2.69 \%$ during grid-connected condition.

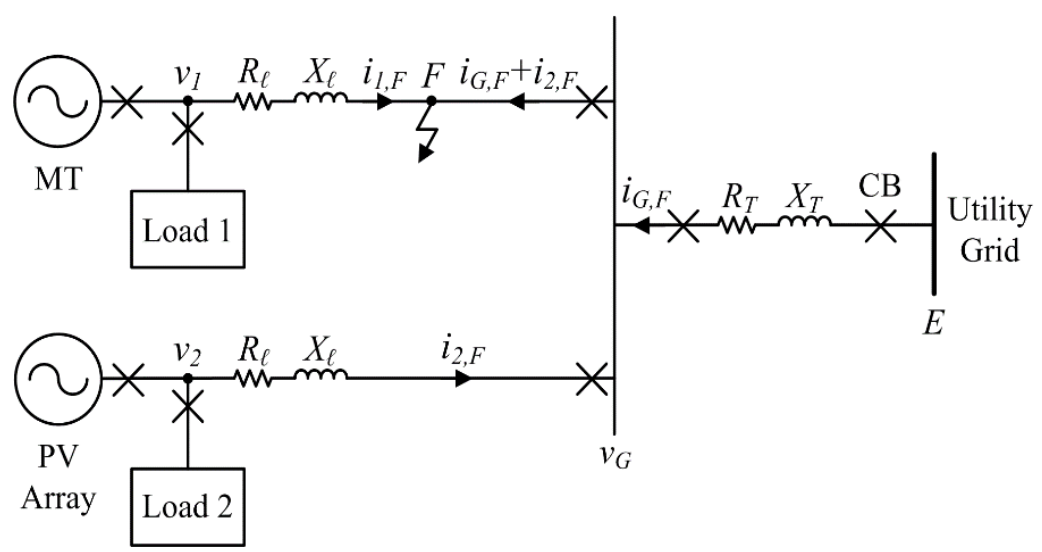

Figure 3-19: Equivalent single-line diagram of the proposed microgrid during fault analysis.

Table 3-2: Numerical Values of Currents and Voltages during Fault

\begin{tabular}{ll}
\hline \hline \multicolumn{1}{c}{ Parameter } & \multicolumn{1}{c}{ Value (p.u.) } \\
\hline Distribution grid voltage (high-voltage side) & $E=1 \angle 0.0402^{\circ}$ \\
Distribution grid voltage (low-voltage side) & $v_{G}=1 \angle 0^{\circ}$ \\
Output voltage of DG inverter 1 & $v_{1}=0.9997 \angle-0.0217^{\circ}$ \\
Output voltage of DG inverter 2 & $v_{2}=0.9998 \angle-0.0145^{\circ}$ \\
Fault current from distribution grid & $i_{G, F}=251 \angle-77.81^{\circ}$ \\
Fault current from DG inverter 1 (MT) & $i_{1, F}=2 \angle-47.14^{\circ}$ \\
Fault current from DG inverter 2(PV array) & $i_{2, F}=2 \angle-47.13^{\circ}$ \\
\hline
\end{tabular}

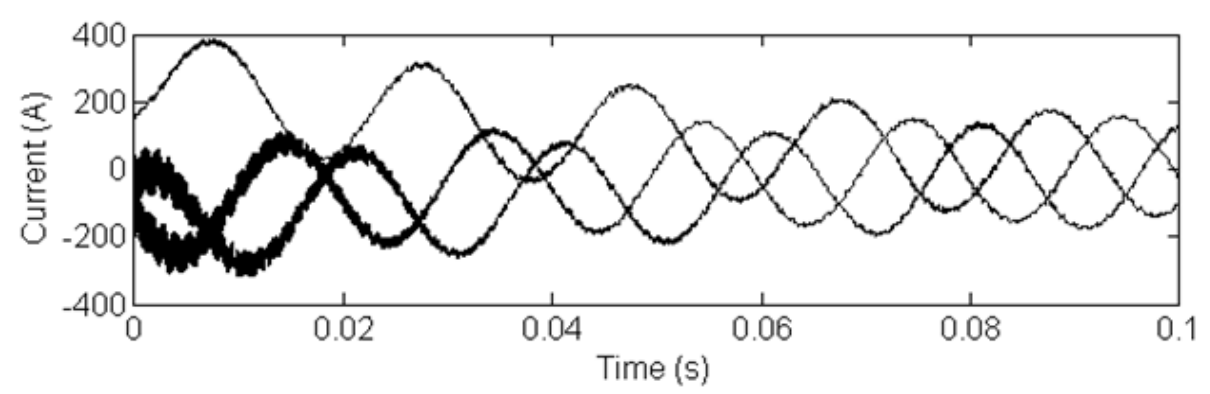

Figure 3-20: Fault current from DG inverter 1. 


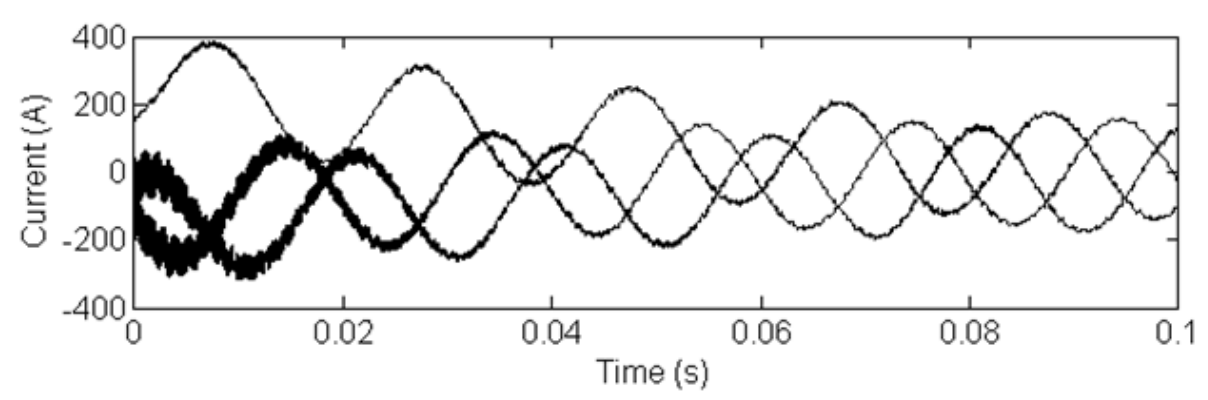

Figure 3-21: Fault current from DG inverter 2.

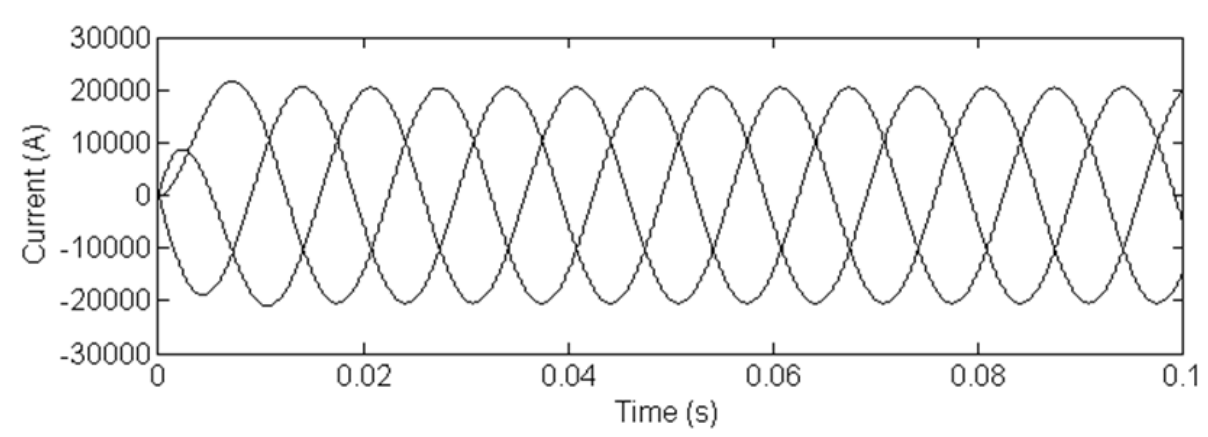

Figure 3-22: Fault current from distribution grid.

To reduce the fault level, one possible solution is simply to improve the short-circuit capabilities of the $\mathrm{CB}$ and distribution lines. However, this will cause an increase in the cost of constructing this microgrid. Another method is to introduce artificial reactances between the DG units and the distribution network. However, due to time constraints, this point will not be discussed in this thesis.

\subsection{Concluding Remarks}

In this chapter, a centralized control approach using an energy management strategy and an MPC based algorithm for the parallel operation of the DG inverters in an AC microgrid in both grid-connected and islanded conditions has been proposed. During grid-connected condition, the operation of the inverters is controlled to work in the $\mathrm{CCM}$ to control the real and reactive power to be delivered to the load. During islanded condition, the inverters then are controlled to work in VCM to regulate the voltage outputs of the DG inverters. The control strategy proposed in the EMS regulates the operation of the paralleled DG inverters, especially during islanded conditions, to use frequency droop control for power sharing. The proposed MPC algorithm decomposes the control problem of the DG inverter into two subproblems. 
Kalman filters are integrated to calculate certain required references for the MPC algorithm based local DG inverter controller. This centralized operation and control approach is incorporated in the simulation of four test cases. The simulation results verify the effectiveness of the control approach. The alteration of the voltage profiles and fault levels are investigated using the proposed microgrid. Further studies can be extended to solve these problems in the future. As this research purely relies on the simulation results, experimental studies using hardware to validate the proposed control approach are needed. 


\section{CHAPTER 4 OPERATION AND CONTROL OF DISTRIBUTED GENERATION INVERTERS IN AN ISLANDED MICROGRID [125]}

\section{Nomenclature}

$\Delta f$

$\Delta P_{\text {apg }}$

$\Delta P_{a b g}$

$\Delta P_{p g}$

$\Delta P_{b g}$

$\Delta P_{L}$

D

H

$f_{0}$

$K_{d p}$

$P_{0}$

$f_{d}$

$P_{\text {cap }}$

$T_{d}$

$P_{\text {diff }}$

$P_{b g 1 \_o p}$

$P_{b g 2 \_o p}$

$P_{b g 1 \_c a p}$

$P_{b g 2 \_c a p}$

$E_{b g 1}$

$E_{b g 2}$

$\mathbf{v}_{s}$

$L_{f}$

$C_{f}$

$\mathbf{i}_{k}$

$\mathbf{i}_{D G}$

Change in the system frequency.

Change in the actual real power generation of the primary DG unit.

Change in the actual real power generations of the backup DG units.

Reference value of the change in the real power of the primary DG unit.

Reference values of the changes in the real power of the backup DG units.

Change in the real power delivered to the load.

Emulated load damping constant of the system.

Emulated inertia constant of the generator.

Nominal system frequency.

Droop control setting.

Nominal real power output of the backup DG units.

Threshold frequency for the backup DG units to activate droop control.

Capacity of each backup DG unit.

Time interval to activate secondary frequency control.

The power difference between the real power delivered by the primary DG unit and the load.

Optimal real power generation reference for backup DG unit 1.

Optimal real power generation reference for backup DG unit 2.

Capacity of backup DG unit 1 .

Capacity of backup DG unit 2.

Relative economic factor of backup DG unit 1.

Relative economic factor of backup DG unit 2.

Output three-phase voltage of the inverter.

Inductance of the LC filter of the inverter.

Capacitance of the LC filter of the inverter.

Three-phase current flowing through $L_{f}$.

Three-phase output current of the inverter. 
$\mathbf{v}_{D G} \quad$ Three-phase output voltage of the inverter.

$\Delta f_{l s} \quad$ Difference between the actual frequency of the microgrid and the allowable lower frequency limit.

$\Delta f_{\text {th }} \quad$ Predetermined frequency deviation threshold for load shedding.

$\Delta P_{t h} \quad$ Real power shedding step.

$K_{l s} \quad$ Load shedding coefficient.

\subsection{Introduction}

In traditional power systems which consist mainly of rotating synchronous generators, any power imbalance between generation and demand will cause the generators to change their operating speed, thus resulting in system frequency variation. However, an islanded microgrid which consists of only inverter-interfaced DG units will exhibit a non-inertia characteristic, and the controllers of the DG inverters will maintain the frequency of the microgrid at the predetermined reference. Because of this, during islanded condition, although there is a difference between generation and demand, the frequency will only fluctuate within acceptable range. Thus, the primary DG unit will suffer from overloading.

To analyze and propose a feasible control strategy for an islanded AC microgrid with inverter-interfaced DG units, a combined centralized and decentralized approach is discussed in this chapter. The key research aspects are listed as follows:

1) Analysis is conducted in this research to model the system frequency of a microgrid. A synthetic frequency model is proposed to be implemented in the EMS for droop control in islanded condition.

2) A droop control based MPC algorithm is proposed for the DG inverters in the microgrid. The MPC algorithm uses a fixed set of equations in both gridconnected and islanded conditions.

3) A control strategy is proposed in the EMS to sustain the operation of a microgrid in both grid-connected and islanded modes. The proposed control strategy also includes a load shedding scheme. 


\subsection{Microgrid Configuration under Study}

This research work focuses on the analysis of a microgrid with inverter-interfaced DG units. This kind of microgrid is not designed to be working in islanded condition for too long with certain percentage of RES penetration. The microgrid configuration for this research is proposed as shown in Figure 4-1. In the configuration, the PCC is connected to a $22 \mathrm{kV}$ infeed through a 1 MVA $22 / 0.4 \mathrm{kV}$ transformer. The PV arrays installed are connected to the PCC through a DC/DC boost converter and a DG inverter. The DC/DC boost converter stabilizes the voltage across the capacitor $C_{p v 2}$, and the DG inverter controls the output current and voltage during grid-connected and islanded conditions. All the line resistances and reactances are neglected because all the DG units and the local load are located within a relatively small area. In this research, the PV is assumed to work in normal condition during islanding because one of the objectives of this chapter is to investigate the implementation of frequency control when the upstream network is disconnected.

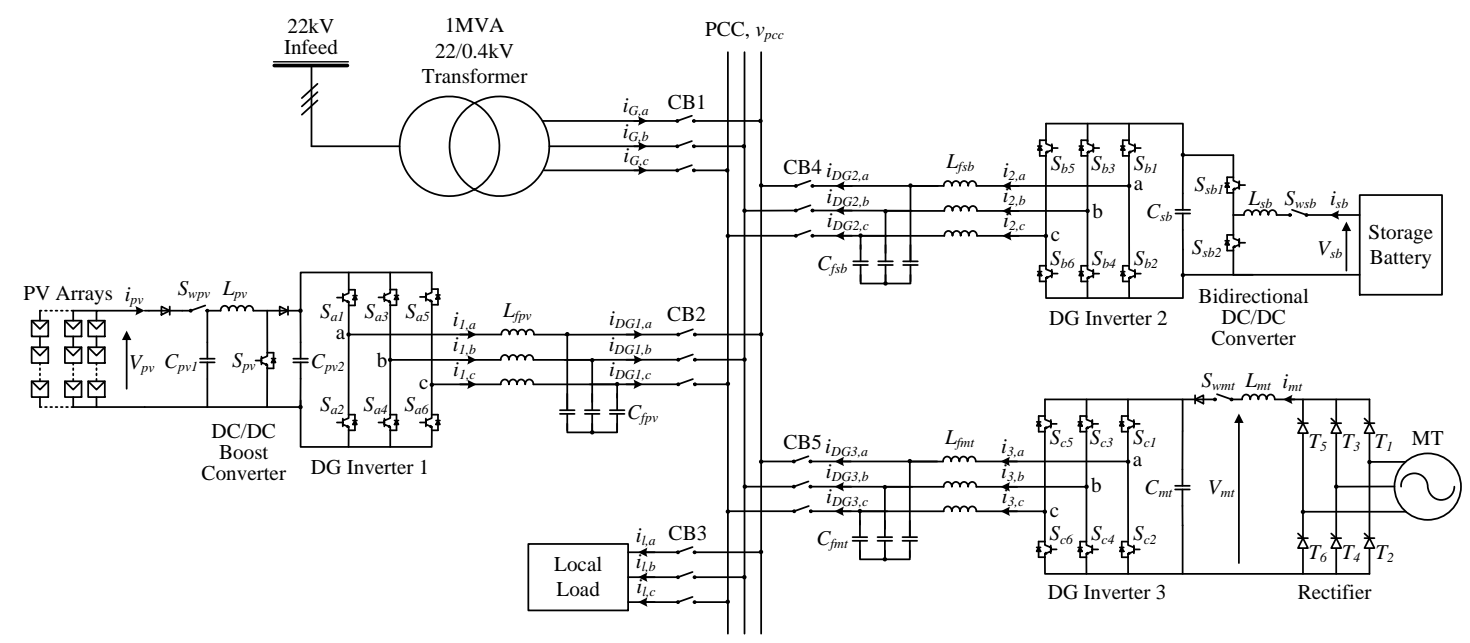

Figure 4-1: Proposed microgrid configuration under study.

Together with the local load, there are two more DG units which are connected to the PCC. The reason to choose SB and MT is that they have very fast response capability. The SB is connected to the PCC through a bidirectional DC/DC converter and a DG inverter. In discharging mode, the bidirectional DC/DC converter stabilizes the voltage across the capacitor $C_{s b}$, and the DG inverter controls the output current and voltage during grid-connected and islanded conditions. In charging mode, the bidirectional DC/DC converter controls the charging current or charging voltage of the SB under 
different charging modes, and the DG inverter is working as a rectifier to stabilize the voltage across the capacitor $C_{s b}$. However, in this research, the charging mode of the SB is not considered because the focus is on the analysis of the microgrid during islanded situation when the SB is working in discharging mode. The SOC of the SB has the following energy constraints: $\mathrm{SOC}_{\min } \leq \mathrm{SOC} \leq \mathrm{SOC}_{\max }$. Under certain situation, if the PV output is higher than the load, the SB will be charged to absorb the overproduction from the PV provided the SOC of the SB is lower than its maximum value. If the SB is fully charged, the excessive PV power generation will be curtailed by the DG inverter of the PV.

The SB can be charged at grid-connected condition during off-peak periods. In this research, it is assumed that at the start of the islanding, the SOC of the SB is at least sufficient to supply power to the microgrid for the duration of the simulation.

Similarly, the MT is connected to the PCC through a rectifier and a DG inverter. The rectifier stabilizes $V_{m t}$, and the DG inverter controls the output current and voltage during grid-connected and islanded conditions.

\subsection{Modeling and Control of Frequency in Microgrid during Islanded Condition}

As described in Section 2.2, $P-f$ droop control and secondary frequency control are largely implemented in traditional power systems because they are a very effective way of controlling different online generators. The frequency is naturally related to the rotational speeds of the online generators which vary according to the supply/demand of power in traditional power systems. However, in an islanded inverter feed microgrid, this relationship between the frequency and the supply/demand of power does not exist anymore.

Therefore, in order to implement a control strategy in a microgrid in both gridconnected and islanded conditions, a frequency model is proposed for the EMS of the proposed microgrid to emulate the frequency characteristics of a synchronous generator during islanded condition. With the implementation of the frequency model, different frequency control methods which consist of $P-f$ droop control and secondary 
frequency control can therefore be proposed for the islanded microgrid. The $P-f$ droop control is used to balance real power generation and demand, while the secondary frequency control is used to restore nominal frequency.

\subsubsection{Frequency Model}

To emulate the frequency behavior of a synchronous generator during islanded condition, a frequency model, which is shown in Figure 4-2, is proposed in this research. Apart from the frequency control methods that will be introduced later, the frequency model can be expressed as follows which are extracted from LFC equations [65]:

$$
\begin{gathered}
\Delta f(s)=G_{P}(s)\left[\Delta P_{a p g}(s)+\Delta P_{a b g}(s)-\Delta P_{L}(s)\right] \\
G_{p g}(s)=\frac{\Delta P_{a p g}}{\Delta P_{p g}}=\frac{1}{1+s T_{p g}} \\
G_{b g}(s)=\frac{\Delta P_{a b g}}{\Delta P_{b g}}=\frac{1}{1+s T_{b g}} \\
G_{P}(s)=\frac{f_{0}}{f_{0} D+2 s H}=\frac{1 / D}{1+s\left(2 H / f_{0} D\right)}=\frac{K_{P}}{1+s T_{P}}
\end{gathered}
$$

where $\Delta f$ represents the change in the system frequency, $G_{P}$ represents the transfer function from real power to system frequency, $\Delta P_{a p g}$ represents the change in the actual real power generation of the primary DG unit, $\Delta P_{a b g}$ represents the change in the actual real power generation of the backup DG units, $\Delta P_{p g}$ represents the reference value of the change in the real power of the primary DG unit, $\Delta P_{b g}$ represents the reference values of the changes in the real power of the backup DG units, $\Delta P_{L}$ represents the change in the real power delivered to the load, $G_{p g}$ represents the transfer function from $\Delta P_{p g}$ to $\Delta P_{a p g}, T_{p g}$ represents the time delay constant in the transfer function $G_{p g}, G_{b g}$ represents the transfer function from $\Delta P_{b g}$ to $\Delta P_{a b g}, T_{b g}$ represents the time delay constant in the transfer function $G_{b g}, D$ represents the emulated load damping constant of the system, $H$ represents the emulated inertia constant of the generator, $f_{0}$ represents the nominal system frequency, and $K_{P}$ and $T_{P}$ are the gain and time constant of the transfer function respectively. 
The emulated inertia parameters, $D$ and $H$ mentioned above, in the frequency model aim to emulate the inertia characteristics of a synchronous generator. There are a few methods to estimate these inertia parameters [126]-[128], which make it feasible to implement the frequency model in the EMS.

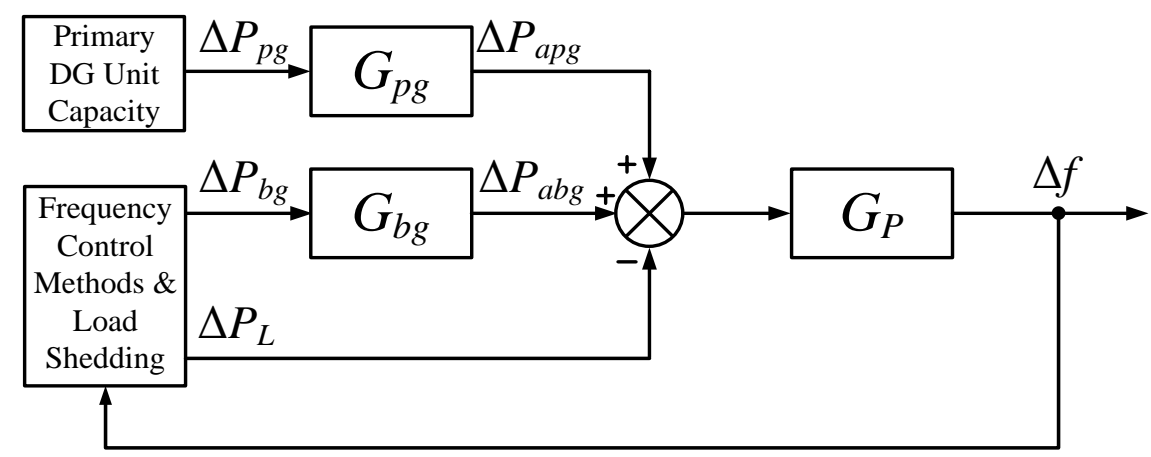

Figure 4-2: Frequency model of an islanded microgrid.

The frequency model is critical for this research because the control strategy is based on frequency control based algorithm which will be implemented to handle islanded situation in an inverter-interfaced microgrid. Without the frequency model, which can be seen from Figure 4-3(a), the frequency of an islanded microgrid will not follow the frequency characteristics of a synchronous generator. This is because the inverter controller can maintain the frequency of the islanded microgrid even when the generation is less than the demand which will cause overloading to the DG units. With the frequency model, which can be seen from Figure 4-3(b), the frequency will adopt the frequency characteristics of a synchronous generator in which the frequency drops when the generation is less than the demand. Load shedding is usually initiated in traditional power systems to restore the frequency back to its nominal value. This avoids overloading to the DG units. 


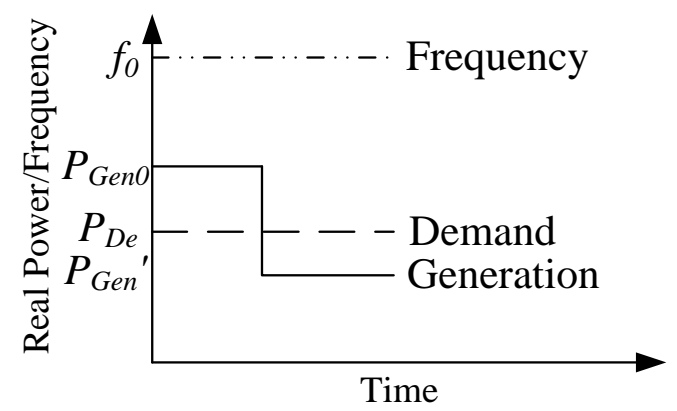

(a)

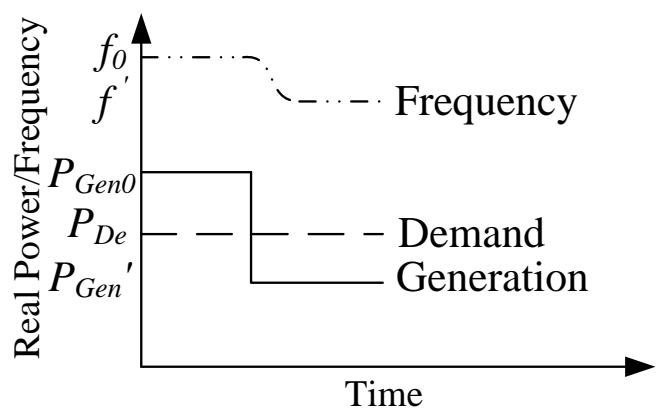

(b)

Figure 4-3: Relationship between frequency and real power: (a) without frequency model; (b) with frequency model.

\subsubsection{P-f Droop Control}

During grid-connected condition, the online generators have a frequency characteristic which is usually acquired through historical data. The inverters of all DG units will adopt a certain $P-f$ droop for droop control.

During islanded condition, with the frequency model described above, the DG inverter of one chosen primary DG unit such as PV, will be provided with the reference of the calculated frequency according to (4.1)-(4.4). It will be responsible for maintaining the frequency during islanded condition.

For the other backup DG units such as SB and MT, $P-f$ droop control is proposed as shown in Figure 4-2. By detecting the frequency in the microgrid, the backup DG units will adjust their real power outputs according to the droop algorithm expressed as follows:

$$
\begin{gathered}
P=\left\{\begin{array}{l}
P_{0}, \text { if } f \geq f_{d} \\
P_{0}-K_{d p}\left(f-f_{d}\right), \text { if } f \leq f_{d}
\end{array}\right. \\
\text { subject to: } P \leq P_{\text {cap } .} .
\end{gathered}
$$

where $K_{d p}$ represents the droop control setting, $P_{0}$ represents the nominal real power output of the backup DG units which is set to be zero initially and will be defined by the secondary frequency control continuously, $f$ is the frequency of the microgrid, $f_{d}$ is 
the threshold frequency for the backup DG units to activate droop control, and $P_{c a p}$ is the capacity of each backup DG unit.

By implementing the above control strategy with primary DG unit maintaining microgrid frequency and backup DG units working in droop control, the power imbalance problem can be solved. However, the system will operate in underfrequency operation. In order to restore the frequency back to its nominal value of $50 \mathrm{~Hz}, \mathrm{a}$ secondary frequency control needs to be incorporated.

\subsubsection{Modified Secondary Frequency Control}

Secondary frequency control is usually performed using automatic generation control (AGC) in traditional power systems. In this research, a modified secondary frequency control for a microgrid in islanded condition is proposed. The time interval $T_{d}$ for traditional AGC is usually 20 seconds to 10 minutes. However, longer time interval will result in longer underfrequency operation time. Therefore, in the microgrid under study, $T_{d}$ is chosen to be 10 seconds for faster response and reasonable computation burden.

After a delay of $T_{d}$, the power difference $P_{\text {diff }}$ between the real power delivered by the primary DG unit and the load is calculated as follows:

$$
P_{\text {diff }}=P_{p g}-P_{L}
$$

The EMS will compare $P_{\text {diff }}$ with the total capacity of the backup DG units, and shed excessive load if necessary. Through the EMS, the references for different backup DG units will then be determined by solving the optimization problem to minimize the following cost function $H_{s}$ : 


$$
\begin{aligned}
H_{s}= & W_{P}\left\{\left[P_{b g 1_{-} o p}-P_{d i f f} P_{b g 1_{-} c a p} /\left(P_{b g 1_{-} c a p}+P_{b g 2_{-} c a p}\right)\right]^{2}\right. \\
& \left.+\left[P_{b g 2_{\_} o p}-P_{d i f f} P_{b g 2_{-} c a p} /\left(P_{b g 1_{-} c a p}+P_{b g 2_{-} c a p}\right)\right]^{2}\right\} \\
& +W_{C}\left(E_{b g 1} P_{b g 1_{-} o p}+E_{b g 2} P_{b g 2_{-} o p}\right)
\end{aligned}
$$

where $W_{P}$ and $W_{C}$ are different weights, $P_{b g 1_{-} o p}$ is the optimal real power generation reference for backup DG unit $1, P_{b g 2}$ op is the optimal real power generation reference for backup DG unit 2, $P_{b g 1 \_c a p}$ is the capacity of backup DG unit $1, P_{b g 2}$ cap is the capacity of backup DG unit 2, and $E_{b g 1}$ and $E_{b g 2}$ are the relative economic factors of backup DG unit 1 and backup DG unit 2 respectively.

The control signals will be sent to the inverters of the backup DG units to reset their generation references. Therefore, the system frequency of the microgrid will be restored back to its nominal value of $50 \mathrm{~Hz}$ by the proposed secondary frequency control.

\subsection{Proposed MPC Algorithm for DG Inverters}

MPC has been a promising control algorithm for many applications, and it is also a very powerful control algorithm for inverters due to its many advantages. As a digital control algorithm, MPC is suitable for power electronic devices. Although the computation burden of MPC algorithm is higher than other simpler control algorithms, the FSC-MPC algorithm proposed in [93], [129] can reduce the computation burden of the traditional MPC which is performed by using quadratic programming. Therefore, MPC is chosen to be the control algorithm for the DG inverters in this research, and (2.9)-(2.13) are applicable in the MPC algorithm.

In [93], [129] the MPC algorithm uses one-step scheme which means it only calculates and executes one time step control signals. Unlike other implementations of MPC, the authors prove that one-step scheme is sufficient for VCM in inverter control. However, when it comes to CCM which is used in islanded condition, a one-step scheme is not sufficient to ensure that the output current follows the reference. 
In this research, the modeling of the inverter is determined by applying Kirchhoff's current and voltage laws to the inverter model in Figure 4-4, the following equations can be obtained:

$$
\begin{gathered}
\mathbf{v}_{s}=\mathbf{i}_{k} R_{\text {loss }}+L_{f} \frac{d \mathbf{i}_{k}}{d t}+\mathbf{v}_{D G} \\
\mathbf{i}_{k}=C_{f} \frac{d \mathbf{v}_{D G}}{d t}+\mathbf{i}_{D G}
\end{gathered}
$$

where $R_{\text {loss }}$ represents the internal loss of the inverter, $L_{f}$ and $C_{f}$ are the inductance and capacitance of the LC filter respectively, $\mathbf{i}_{k}$ is the three-phase current which flows through $L_{f}$, and $\mathbf{i}_{D G}$ and $\mathbf{v}_{D G}$ are the three-phase output current and voltage of the inverter respectively.

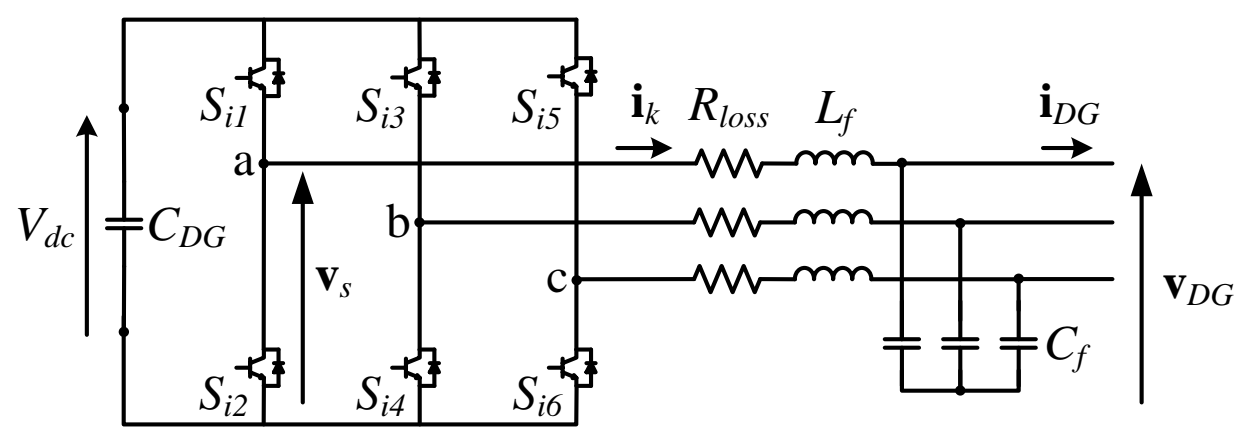

Figure 4-4: Three-phase DG inverter model.

Compared to the sampling frequency, the output current $\mathbf{i}_{D G}$ is changing slowly. Therefore, the following assumption is made:

$$
\frac{d \mathbf{i}_{D G}}{d t}=0
$$

By rearranging (4.8)-(4.10) into a state-space form, the continuous-time state-space model can be obtained as follows:

$$
\frac{d \mathbf{x}}{d t}=\mathbf{A x}+\mathbf{B u}
$$




$$
\mathbf{y}=\mathbf{C x}
$$

where $\mathbf{x}=\left[\begin{array}{lll}\mathbf{i}_{k} & \mathbf{v}_{D G} & \mathbf{i}_{D G}\end{array}\right]^{\mathrm{T}}, \mathbf{y}=\left[\begin{array}{ll}\mathbf{i}_{D G} & \mathbf{v}_{D G}\end{array}\right]^{\mathrm{T}}, \mathbf{u}=\mathbf{v}_{s}$,

$$
\mathbf{A}=\left[\begin{array}{ccc}
-R_{\text {loss }} / L_{f} & -1 / L_{f} & 0 \\
1 / C_{f} & 0 & -1 / C_{f} \\
0 & 0 & 0
\end{array}\right], \mathbf{B}=\left[\begin{array}{c}
1 / L_{f} \\
0 \\
0
\end{array}\right], \mathbf{C}=\left[\begin{array}{lll}
0 & 0 & 1 \\
0 & 1 & 0
\end{array}\right]
$$

The continuous state-space model described in (4.11) and (4.12) is then discretized using zero-order hold with a sampling time of $T_{s}=5 \times 10^{-6} \mathrm{~s}$. The discrete state-space model can be obtained as follows:

$$
\begin{gathered}
\mathbf{x}(k+1)=\mathbf{A}_{d} \mathbf{x}(k)+\mathbf{B}_{d} \mathbf{u}(k) \\
\mathbf{y}(k)=\mathbf{C}_{d} \mathbf{x}(k)
\end{gathered}
$$

where $\mathbf{A}_{d}, \mathbf{B}_{d}$ and $\mathbf{C}_{d}$ are discretized coefficients from $\mathbf{A}, \mathbf{B}$ and $\mathbf{C}$ in (4.11) and (4.12) using zero-order hold and sampling time $T_{s}$.

In [93], [129], the reference is used to determine the control signal $\mathbf{u}(k)$. Technically, with more steps of reference ahead of time to be considered, the control signal $\mathbf{u}(k)$ can be more accurate because of more information. However, having more steps of reference will result in an exponential increase in computational burden. Therefore, in this research, two steps of reference are used to ensure better control performance, while ensuring a reasonable computational burden. This point will also be verified in the case studies in Section 4.6 later. The controller obtains the control signals by minimizing the following cost function which tracks the error between the references and the outputs:

$$
\begin{gathered}
J=\{\mathbf{W}[\mathbf{r}(k)-\mathbf{y}(k)]\}^{\mathrm{T}}[\mathbf{r}(k)-\mathbf{y}(k)] \\
+\{\mathbf{W}[\mathbf{r}(k+1)-\mathbf{y}(k+1)]\}^{\mathrm{T}}[\mathbf{r}(k+1)-\mathbf{y}(k+1)] \\
\text { subject to } \mathbf{v}_{D G}=V_{d c} \mathbf{S}
\end{gathered}
$$


where $\mathbf{r}(k)$ is the reference matrix which includes reference current and voltage, $\mathbf{W}=\left[\begin{array}{cc}W_{i} & 0 \\ 0 & W_{v}\end{array}\right]$ is a weight matrix which adds different weights to the output current and voltage. By assigning a larger value to one of these two weights, the controller will give more effort in controlling output current (if $W_{i}$ is larger) or output voltage (if $W_{v}$ is larger).

It can be observed that the MPC algorithm uses a fixed set of equations in both gridconnected and islanded conditions. To ensure proper operation of the DG inverters during both operating conditions, both the output current and voltage are controlled. By choosing different sets of weights for the output current and voltage in (4.15), the controller can adjust itself into two different operation modes, i.e., VCM and CCM.

\subsection{Proposed Control Strategy in the EMS}

The objective of this research is to propose a control strategy for an islanded microgrid with inverter-interfaced DG units. To take advantages of the frequency control methods and the proposed MPC algorithm discussed in the previous sections, a complete control strategy is proposed in the EMS. With proper control strategy in the EMS, the control methods and algorithm are able to guarantee the operation of the microgrid in both operation modes.

Figure 4-5 illustrates the proposed control strategy that the EMS adopts for the microgrid under study. The EMS will detect and measure the energy and power information of the microgrid. If the microgrid stays in grid-connected condition, all the inverters will operate in droop control when the reference voltages and currents are calculated using droop algorithm in (4.5) with current and voltage measurements. 


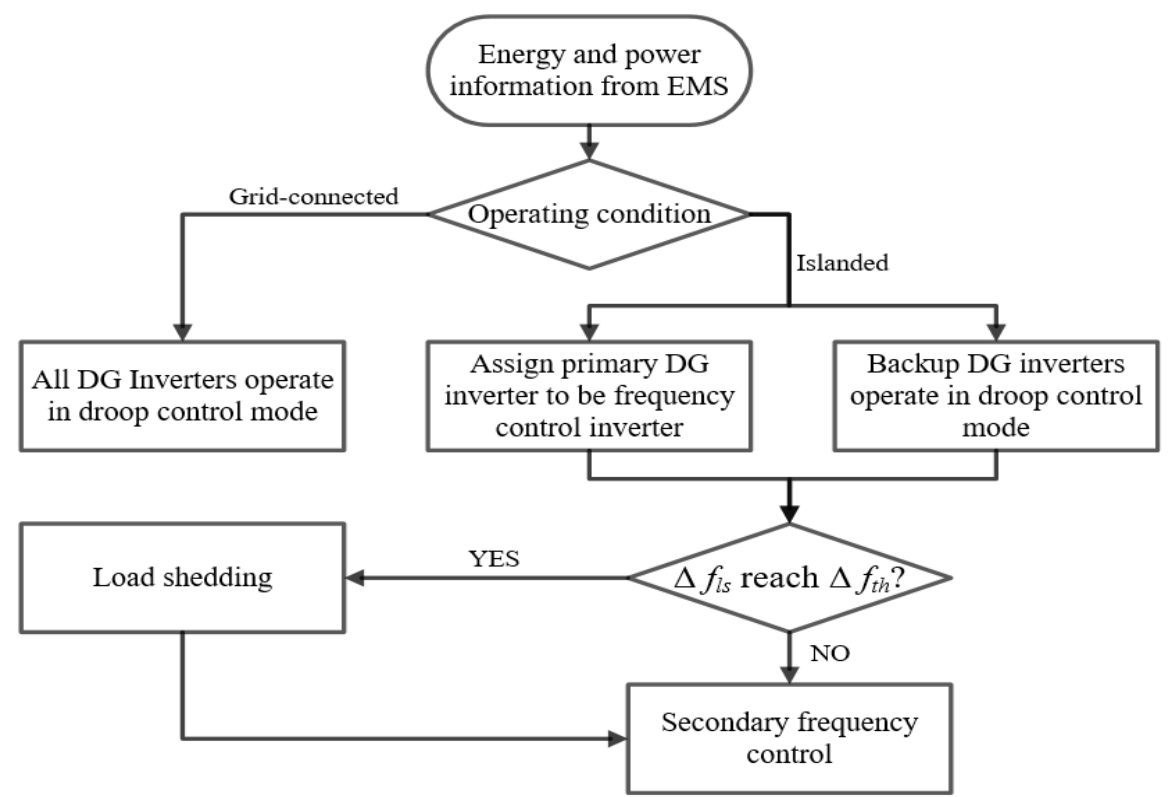

Figure 4-5: Proposed control strategy adopted by the EMS.

If the microgrid starts to be islanded from the upstream network, the microgrid will work in islanded condition with a delay. Though smart meters and power electronic devices such as inverters in the microgrid can respond in a very short time, a time delay $T_{d l}$ is still inevitable because of the signal transmission and the processing in the EMS. During $T_{d l}$, the inverters will operate as if they are still in grid-connected condition. However, in this research, both the output current and voltage are controlled as shown by (4.15), thus the operation of the microgrid can still be maintained during this period.

After $T_{d l}$, the microgrid will work in islanded condition. As illustrated in Figure 4-5, during islanded condition, one inverter will be chosen by EMS to control the frequency. The reference of this inverter will be calculated using the frequency model in (4.1) to (4.4) to work in VCM (in this research, VCM means the weight of the reference voltage in the cost function is larger than that of the reference current). The references of other inverters will be still calculated using droop algorithm in (4.5) with current and voltage measurements just like in grid-connected mode.

However, due to different loading conditions, the power supplied by the SB and MT may not be sufficient to meet the power demand of the islanded microgrid. Therefore, as illustrated in Figure 4-5, when the frequency drops until the frequency difference 
$\Delta f_{l s}$, which is the difference between the actual frequency of the microgrid and the allowable lower frequency limit of $49.8 \mathrm{~Hz}$, reaches a predetermined frequency deviation threshold $\Delta f_{t h}$, the load shedding is activated. This load shedding scheme is proposed as follows:

- Shed $\Delta P_{t h}$ real power of the load demand when $\Delta f_{l s}$ reaches $\Delta f_{t h} . \Delta P_{t h}$ is the predetermined real power shedding step.

- Shed $K_{l s} \times \Delta P_{t h}$ real power of the load demand when $\Delta f_{l s}$ reaches $K_{l s} \times \Delta f_{t h}$. During this period, the non-critical load will be shed.

- Shed $2 K_{l s} \times \Delta P_{t h}$ real power of the load demand when $\Delta f_{l s}$ reaches $2 K_{l s} \times \Delta f_{t h}$. During this period, some of the critical load will be shed.

The economic analysis of the load shedding can be found in [130], [131], which offer possible ways to realize the proposed load shedding schedule with real-time pricing and critical levels of load. The load shedding coefficient $K_{l s}$ is usually defined based on historical data in a certain power system.

After the operations mentioned above, the proposed secondary frequency control will be activated at $t=T_{d}$ to restore the frequency to the nominal value of $50 \mathrm{~Hz}$. All the DG units will continue to supply real power to the islanded microgrid according to the optimal dispatch algorithm as detailed in (4.7).

Note that this microgrid is not designed to be working in islanded mode for too long as it is an on-grid microgrid, grid connection is supposed to be resumed before the islanded microgrid collapses. When grid connection is resumed, the microgrid is assumed to work just as before islanding. This part can be easily realized as the grid is considered as an infinite bus. This research will not engage this process.

For the coordinated control of voltage magnitude, it is essential for the microgrid to compensate the reactive power locally to guarantee the upstream network working at unity power factor. Therefore, at least one of the DG units' capacity will be chosen to be large enough to fully compensate the reactive power consumption of the load by design. During grid-connected and islanded conditions, the reactive power will always be supplied by the DG unit which is large enough to compensate it. This reactive 
power control is more straightforward and effective. Firstly, it does not require a specific controller which saves processing resources. Secondly, by compensating the reactive power all the time will guarantee the power factor of upstream network. Lastly, the proposed MPC algorithm can handle this reactive power control effectively as it only needs calculated references to control the inverters. This approach is validated in Chapter 3.

\subsection{Simulation Studies}

Simulation studies are conducted to analyze the frequency of the islanding of the microgrid with the EMS described above. The configuration of the microgrid under study is shown in Figure 4-1, and the system parameters are given in Table 4-1. It is assumed to be tested during a sunny daytime. The PV, which is always working at maximum power point, is also chosen to be the one to compensate the reactive power and to control the frequency during islanded condition by the EMS.

Table 4-1: Parameters Used in the Simulation Studies

\begin{tabular}{ll}
\hline \hline \multicolumn{1}{c}{ Parameter } & \multicolumn{1}{c}{ Value } \\
\hline Capacity of PV Power Generation & $S_{P V_{-} c a p}=15 \mathrm{kVA}$ \\
Capacity of SB Power Generation & $S_{S B_{-} c a p}=10 \mathrm{kVA}$ \\
Capacity of MT Power Generation & $S_{M T_{-} c a p}=7.5 \mathrm{kVA}$ \\
Relative Economic Factor of the SB & $E_{S B}=1$ \\
Relative Economic Factor of the MT & $E_{M T}=1.5$ \\
Local Load during Light Load & $P_{L_{-} l}=27 \mathrm{~kW}$ \\
& $Q_{L_{-} l}=9 \mathrm{kVAr}$ \\
Local Load during Heavy Load & $P_{L_{-} h}=59 \mathrm{~kW}$ \\
Nominal Voltage at PCC & $Q_{L_{-} h}=12 \mathrm{kVAr}$ \\
Nominal System Frequency & $v_{P C C}=230 \mathrm{~V}$ rms $($ phase $)$ \\
Emulated Inertia Constant & $f_{0}=50 \mathrm{~Hz}$ \\
Emulated Load Damping Constant & $H=3 \mathrm{~s}$ \\
Inverter Internal Loss & $D=10$ \\
Inductance of the LC Filter in Inverter & $R_{l o s s}=0.01 \Omega$ \\
& $L_{f}=1.2 \mathrm{mH}$
\end{tabular}


Capacitance of the LC Filter in Inverter

$C_{f}=20 \mu \mathrm{F}$

Response Time of EMS

$T_{d l}=0.1 \mathrm{~s}$

Time Interval for Secondary Frequency Control

$T_{d}=10 \mathrm{~s}$

Droop Control Activating Frequency for PV

$f_{d} P V=50 \mathrm{~Hz}$

Droop Control Activating Frequency for SB

$f_{d} S B=49.98 \mathrm{~Hz}$

Droop Control Activating Frequency for MT

$f_{d \_} M T=49.90 \mathrm{~Hz}$

Droop Control Setting for PV

$K_{d_{-} P V}=0 \mathrm{~kW} / \mathrm{Hz}$

Droop Control Setting for SB

$K_{d \_} S B=-100 \mathrm{~kW} / \mathrm{Hz}$

Droop Control Setting for MT

$K_{d \_M T}=-100 \mathrm{~kW} / \mathrm{Hz}$

Frequency Deviation Threshold

$\Delta f_{\text {th }}=0.2 \mathrm{~Hz}$

Real Power Shedding Step

$\Delta P_{t h}=5 \mathrm{~kW}$

Load Shedding Coefficient

$K_{l s}=2$

\subsubsection{Test Case 1: Islanding during Light Load Condition}

During light load condition, the real power delivered by the PV in the microgrid is able to supply a high percentage of the local load. In this test case, the real and reactive power consumption of the local load are $27 \mathrm{~kW}$ and $9 \mathrm{kVAr}$ respectively as shown in Table 4-1. Within $15 \mathrm{~s}$, the maximum power point of PV is assumed to be constant.

The real and reactive power delivered by the PV are shown in Figures 4-6 and 4-7 respectively. It can be seen that for $0 \leq t<3 \mathrm{~s}$, the PV DG unit is delivering its maximum power output of $15 \mathrm{kVA}$ with the real power generation of $12 \mathrm{~kW}$ and the reactive power generation of $9 \mathrm{kVAr}$. Therefore, the grid provides the remaining $15 \mathrm{~kW}$ as shown in Figure 4-6, and the reactive power of the load is fully supplied by the PV DG unit shown in Figure 4-7. The real power delivered by the SB and MT are shown in Figure 4-8. The frequency and the root mean square (RMS) values of the PCC voltage are maintained at the nominal values of $50 \mathrm{~Hz}$ and $230 \mathrm{~V}$ respectively, as shown in Figures 4-9 and 4-10 respectively. 


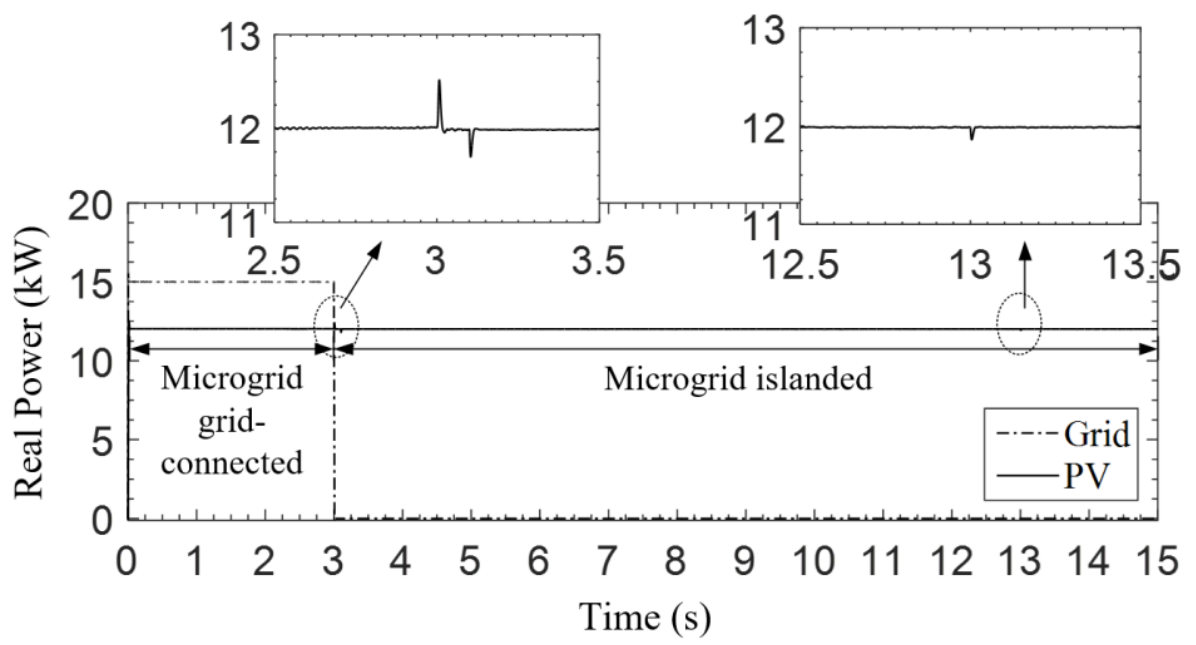

Figure 4-6: Real power delivered by the grid and PV in Test Case 1.

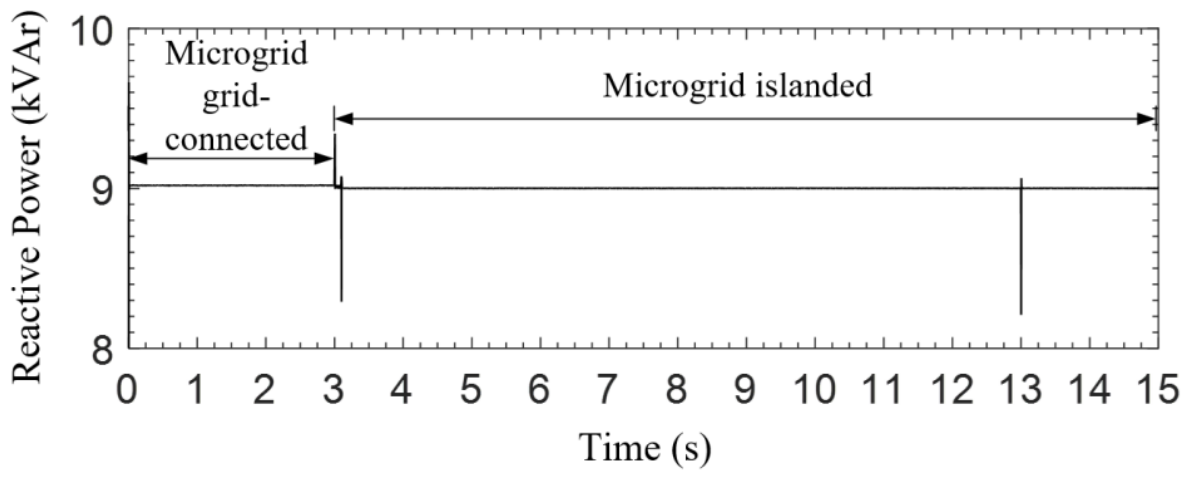

Figure 4-7: Reactive power delivered by the PV in Test Case 1.

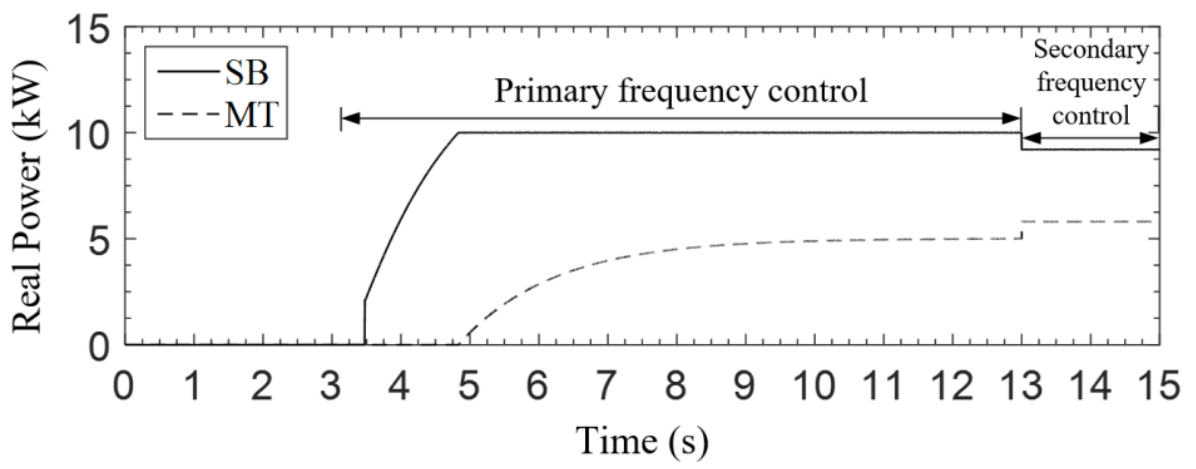

Figure 4-8: Real power delivered by the SB and MT in Test Case 1. 


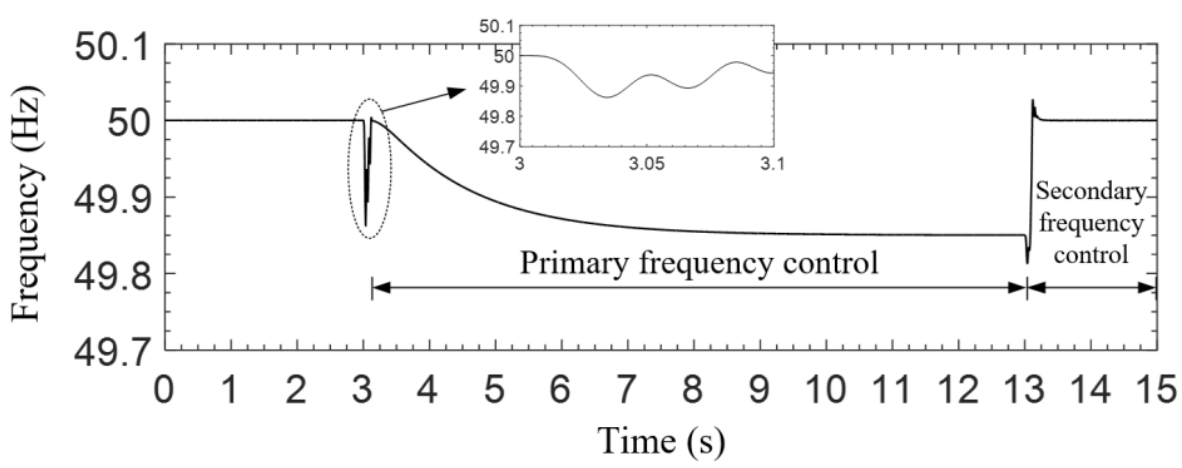

Figure 4-9: Frequency of the microgrid in Test Case 1.

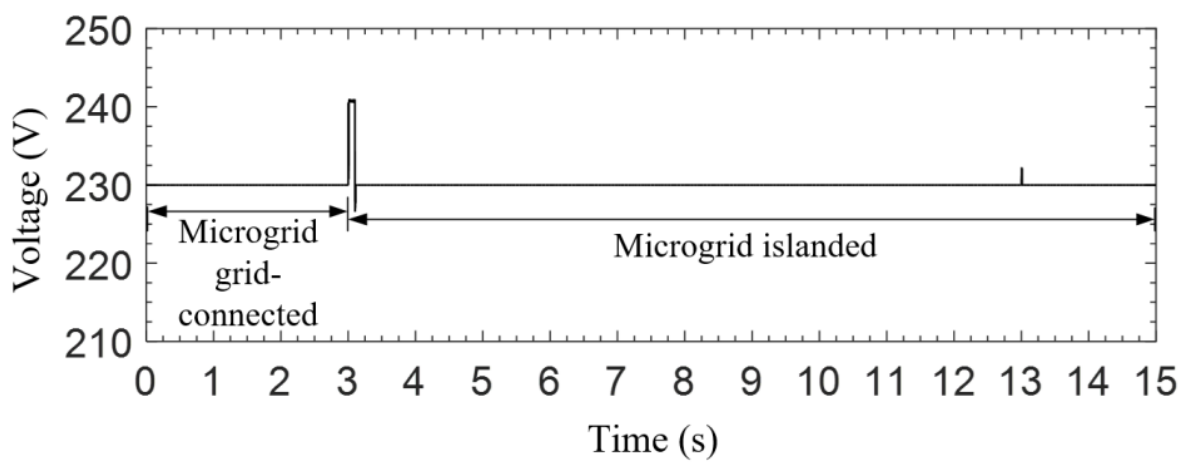

Figure 4-10: RMS value of the PCC voltage in Test Case 1.

From Figure 4-9, it can be seen that the frequency stays at $50 \mathrm{~Hz}$ during gridconnected condition. The power generated by PV and the power supplied from upstream network can supply all the consumption of the load. During this period of 15 $\mathrm{s}$, the PV power generation is assumed to be constant.

At $t=3 \mathrm{~s}$, a fault occurs on the upstream network. As illustrated in Figure 4-1, CB1 trips, isolating the microgrid while sending a tripping signal to the EMS. As shown in Figure 4-6, the real power delivered by the grid immediately decreases to zero. However, as mentioned in Section 4.5, because of $T_{d l}$, for $3 \leq t<3.1 \mathrm{~s}$, the DG inverter of the PV still operates in CCM. With the implementation of the MPC algorithm, the output current and voltage can still be maintained within their acceptable ranges.

It can be seen from Figure 4-9 enlarged frequency waveform for $3.0 \leq t \leq 3.1 \mathrm{~s}$ that without the $P-f$ droop control being activated, the frequency of the microgrid is fluctuating below $50 \mathrm{~Hz}$ but within the acceptable range of $\pm 0.2 \mathrm{~Hz}$ [132]. This is because the real power imbalance in the generation and demand intends to cause the 
frequency to drop, but the control algorithm of the PV inverter still tries to maintain the frequency at the predetermined value of $50 \mathrm{~Hz}$. If the proposed control strategy is not implemented as mentioned in the previous sections, the frequency will continue to fluctuate in a similar pattern, causing the PV DG unit to operate in overloading condition as the generation is less than the demand.

The EMS receives the isolating command at $t=3 \mathrm{~s}$, for $3.1 \leq t \leq 13 \mathrm{~s}$, the microgrid is operating under the proposed control strategy. As described in Section 4.5, from $t=3.1$ $\mathrm{s}$, the frequency of the microgrid is controlled by the DG inverter of the PV. As shown in Figure 4-9, the frequency is controlled to decrease gradually which emulates the effect of the inertia of a synchronous generator. In the meantime, the droop control based MPC algorithm in the SB and MT operates in response to the frequency change of the microgrid. As described in Table 4-1, the SB starts to supply real power to the microgrid when the frequency drops to $49.98 \mathrm{~Hz}$ at $t=3.51 \mathrm{~s}$ as shown in Figure 4-8. The decrement of $0.02 \mathrm{~Hz}$ from the nominal $50 \mathrm{~Hz}$ can avoid the SB being activated during a temporary frequency fluctuation. From Figure 4-8, it can be seen that when the $\mathrm{SB}$ is delivering real power at its maximum output of $10 \mathrm{~kW}$, the frequency of the microgrid continues to decrease as shown in Figure 4-9. Therefore, when the frequency drops to $49.9 \mathrm{~Hz}$ at $t=4.76 \mathrm{~s}$, the MT starts to deliver real power to the microgrid as shown in Figure 4-8. With the MT continuously increasing its real power generation to $5 \mathrm{~kW}$, the frequency of the microgrid controlled by the DG inverter of PV settles down to $49.85 \mathrm{~Hz}$ at $t=9.69 \mathrm{~s}$, which is within the acceptable range of \pm 0.2 $\mathrm{Hz}$ [132] as shown in Figure 4-9. With the proposed frequency control strategy, the reactive power generation of $\mathrm{PV}$ remains at $9 \mathrm{kVAr}$ both in grid-connected and islanded conditions as shown in Figure 4-7. As the reactive power generation and demand in the microgrid is always balanced, the RMS value of the PCC voltage is maintained at $230 \mathrm{~V}$ for $3.1 \leq t \leq 13 \mathrm{~s}$ as shown in Figure 4-10.

The microgrid was islanded at $t=3 \mathrm{~s}$. After $T_{d}=10 \mathrm{~s}$, the proposed secondary frequency control is activated at $t=13 \mathrm{~s}$ to restore the frequency of the microgrid to its nominal value of $50 \mathrm{~Hz}$ as shown in Figure 4-9. The EMS uses the proposed optimal dispatch algorithm (4.7) in Section 4.3 to determine the references of real power delivered by the SB and MT. From Figure 4-8, it can be seen that the SB and MT deliver $9.2 \mathrm{~kW}$ and $5.8 \mathrm{~kW}$ of real power to the microgrid respectively. The RMS value of the PCC 
voltage is still maintained at $230 \mathrm{~V}$, which is shown in Figure 4-10, because the reactive power generation of $\mathrm{PV}$ remains at $9 \mathrm{kVAr}$ as shown in Figure 4-7. The normal operation of the microgrid is therefore restored.

\subsubsection{Test Case 2: Islanding during Heavy Load Condition}

Under heavy load condition, the real power delivered by the PV can only supply $15 \%$ $(9 \mathrm{~kW})$ of the load according to Table $4-1$. The remaining $85 \%(50 \mathrm{~kW})$ of the load is supplied by the grid. For $0 \leq t<3 \mathrm{~s}$, the real and reactive power delivered by the PV are maintained at $9 \mathrm{~kW}$ and $12 \mathrm{kVAr}$ respectively as shown in Figures 4-11 and 4-12 respectively. The real power delivered by the grid is maintained at $50 \mathrm{~kW}$ as shown in Figure 4-11. The SB and MT work at standby mode without any real power output, which can be seen in Figure 4-13. The load stays at $59 \mathrm{~kW}$ which is shown in Figure 4-11. During this period, the frequency of the microgrid is maintained at $50 \mathrm{~Hz}$, shown in Figure 4-14. The RMS value of the PCC voltage will always be kept at $230 \mathrm{~V}$ because of the adopted reactive power control in the PV inverter.

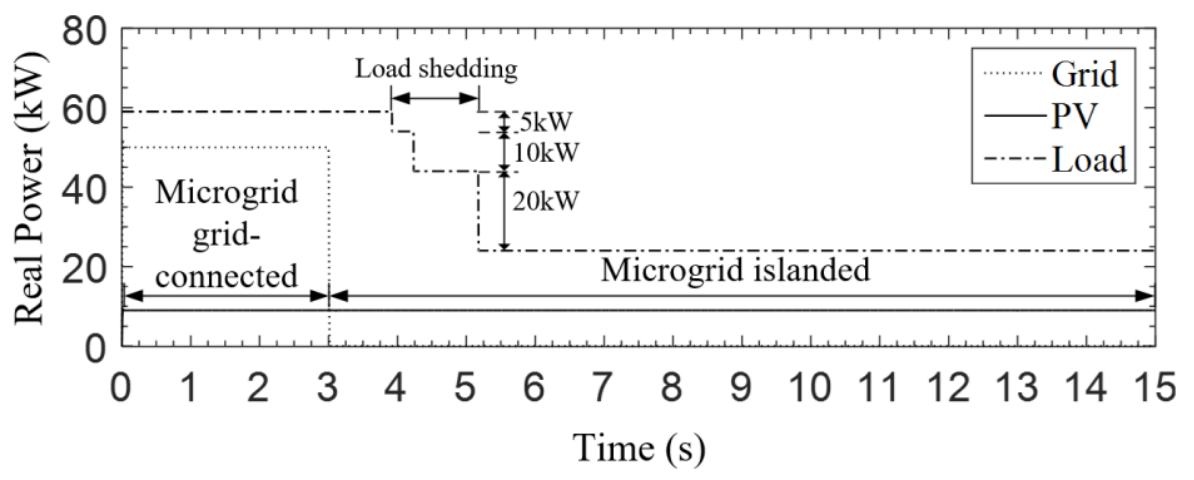

Figure 4-11: Real power delivered by the grid and PV, and load demand in Test Case 2. 


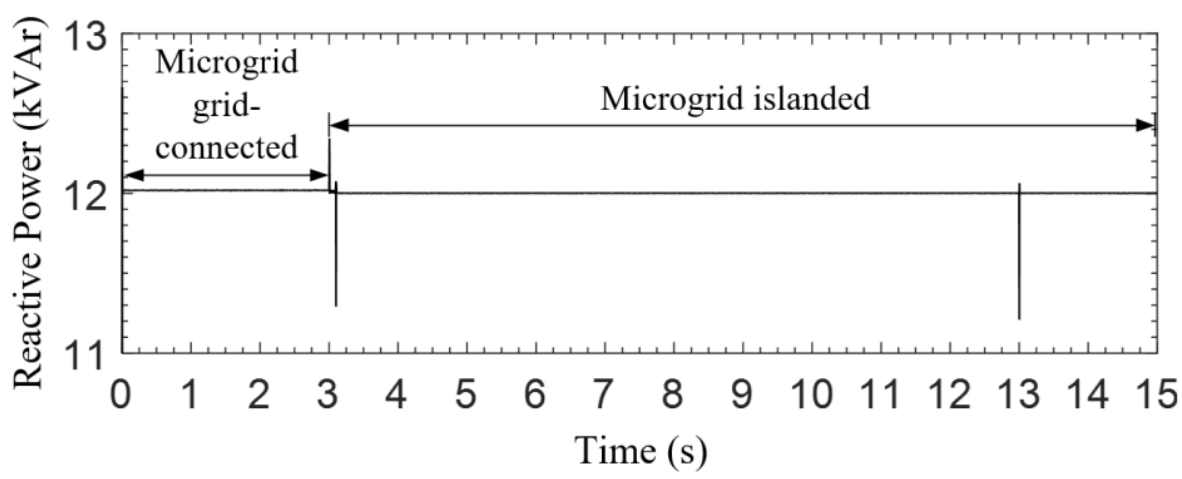

Figure 4-12: Reactive power delivered by the PV in Test Case 2.

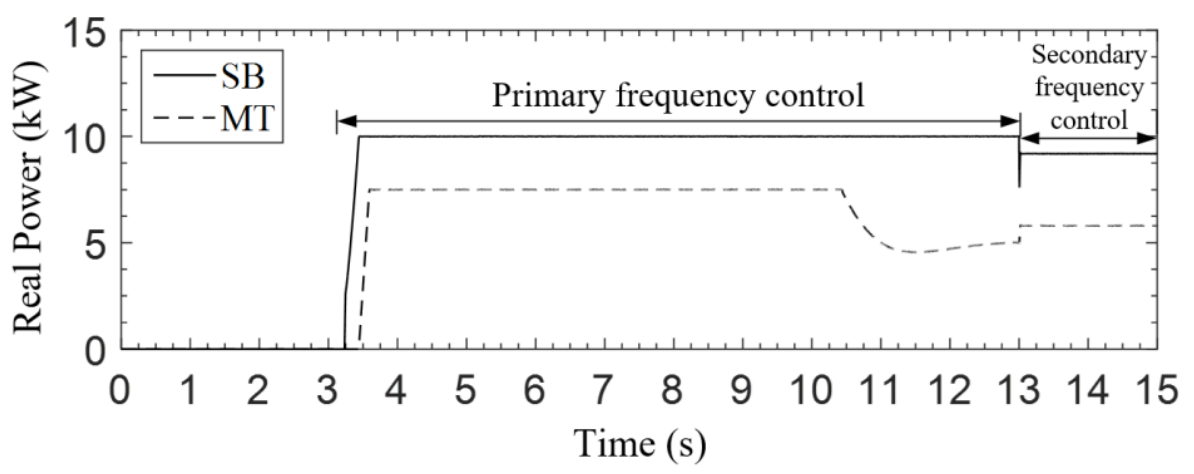

Figure 4-13: Real power delivered by the SB and MT in Test Case 2.

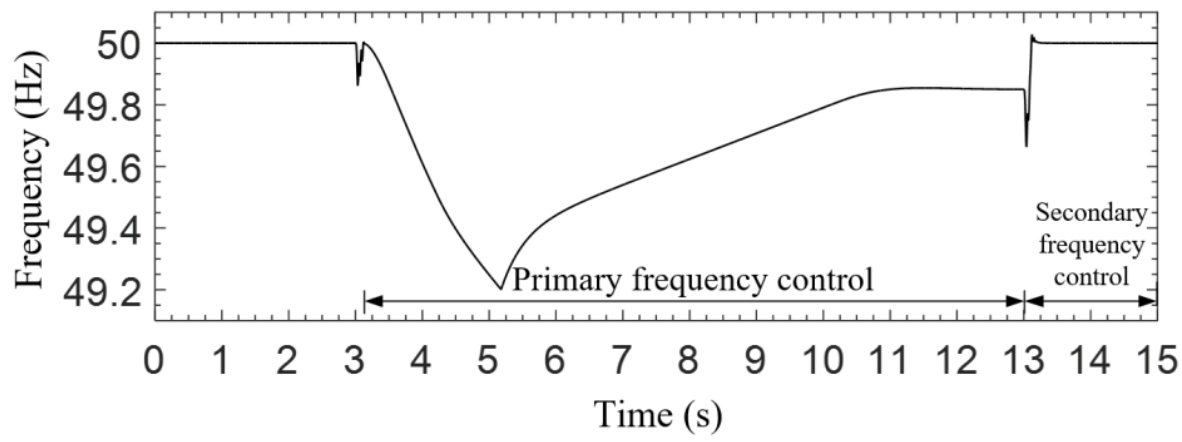

Figure 4-14: Frequency of the microgrid in Test Case 2.

At $t=3 \mathrm{~s}$, a fault occurs on the upstream network. The microgrid operator islands the microgrid to maintain its operation. It can be seen from Figure 4-11 that the real power of $50 \mathrm{~kW}$ delivered by the grid drops to zero immediately. As described in Test Case 1, for $3 \leq t<3.1 \mathrm{~s}$, the DG inverter of PV still works as if it is still grid-connected, while the proposed control strategy is not activated. The SB and MT are not delivering any real power, which can be seen from Figure 4-13. The real power load demand stays at $59 \mathrm{~kW}$ as shown in Figure 4-11. 
It can be seen from Figure 4-14 that the frequency fluctuates below $50 \mathrm{~Hz}$ but within the acceptable range of $\pm 0.2 \mathrm{~Hz}$ [132]. If the proposed control strategy is not implemented in this network, the frequency of the microgrid will continue to fluctuate even with an imbalance between real power supply and demand.

For $3.1 \leq t \leq 13 \mathrm{~s}$, the proposed droop control is activated. The SB starts to supply real power at $t=3.24 \mathrm{~s}$ as shown in Figure 4-13 when the frequency controlled by the DG inverter of PV drops to $f=49.98 \mathrm{~Hz}$ as shown in Figure 4-14. The MT starts to supply real power to the microgrid at $t=3.48 \mathrm{~s}$ when the frequency drops to $49.9 \mathrm{~Hz}$ as shown in Figure 4-13. However, with the real power outputs of all the DG units (i.e., $9 \mathrm{~kW}$ from the PV, $10 \mathrm{~kW}$ from the $\mathrm{SB}$ and $8 \mathrm{~kW}$ from the MT), the generation in the microgrid cannot meet the real power load demand of $59 \mathrm{~kW}$. The frequency of the microgrid, which is controlled by the frequency model, continues to decrease as shown in Figure 4-14. Therefore, load shedding is activated as explained in Section 4.5.

It can be observed from Figure 4-11 that three times of load shedding are performed for $3 \leq t \leq 13 \mathrm{~s}$ according to the load shedding scheme presented in Section 4.5. At $t=3.92 \mathrm{~s}$, the EMS sheds a load of $5 \mathrm{~kW}$ when $f=49.6 \mathrm{~Hz}$; at $t=4.24 \mathrm{~s}$, the EMS sheds a load of $10 \mathrm{~kW}$ when $f=49.4 \mathrm{~Hz}$; and at $t=5.18 \mathrm{~s}$, the EMS sheds a load of $20 \mathrm{~kW}$ when $f=49.2 \mathrm{~Hz}$. After the third load shedding, the frequency of the microgrid is restored to $49.82 \mathrm{~Hz}$ at $t=10.75 \mathrm{~s}$, which is within the acceptable range of $\pm 0.2 \mathrm{~Hz}$ [132]. During the process of restoring the frequency, the MT continues to adjust its operation according to the $P-f$ droop control until the generation meets the load demand as shown in Figure 4-13.

For $3 \leq t \leq 13 \mathrm{~s}$, the reactive power delivered by the PV remains at $12 \mathrm{kVAr}$ as shown in Figure 4-12, which is the reactive power load demand in this test case. Therefore, the PCC voltage can be kept at $230 \mathrm{~V}$ similar to Test Case 1.

At $t=13 \mathrm{~s}$, the proposed secondary frequency control is activated to restore the frequency of the microgrid to $50 \mathrm{~Hz}$ as in Test Case 1. With the implementation of the optimal dispatch algorithm defined in (4.7), the SB and MT deliver $9.2 \mathrm{~kW}$ and 5.8 
$\mathrm{kW}$ of real power to the microgrid respectively. The frequency of the microgrid is then restored back to its nominal value of $50 \mathrm{~Hz}$ as shown in Figure 4-14.

\subsubsection{Test Case 3: Comparison between MPC and PR in SB discharging}

As mentioned previously in Section 2.5, the advantage of MPC algorithm lies in the fact that it is easy for advanced features and complicated centralized control strategies to be included in the EMS as well as the improvements in DG inverter performance.

To verify the improvement in performance, an SB discharging test case is conducted in simulation. The microgrid is working in grid-connected condition, and the EMS sends a signal to the SB to discharge $7.5 \mathrm{~kW}$ to the load in order to meet the demand in a centralized control approach. The DG inverter of the SB is controlled by the proposed MPC algorithm and a fine-tuned PR control algorithm in this test case scenario. The results are observed as shown in Figure 4-15.

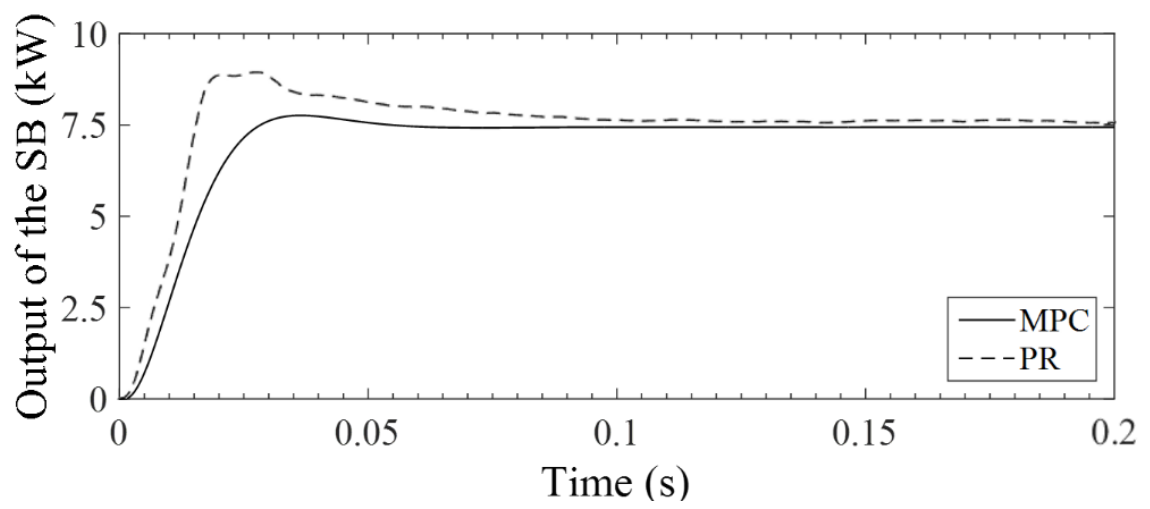

Figure 4-15: Comparison between MPC and PR in real power delivered by the SB in Test Case 3.

From Figure 4-15, it can be observed that:

- Settling time: 3 cycles for the proposed MPC algorithm; 4 cycles for the PR control algorithm.

- Overshoot: $3.3 \%$ for the proposed MPC algorithm; $20 \%$ for the PR control algorithm.

- Tracking error: $\pm 0.2 \%$ for the proposed MPC algorithm; $\pm 1.7 \%$ for the PR control algorithm. 
From this test case, it can be seen that the proposed MPC algorithm has a better performance compared to the conventional PR control algorithm.

\subsection{Concluding Remarks}

In this chapter, an inverter control strategy has been proposed for an islanded microgrid with inverter-interfaced DG units. A frequency model has been proposed to be incorporated into the EMS to emulate the frequency characteristics of a synchronous generator. Frequency control methods, including the proposed $P-f$ droop control and secondary frequency control, have been implemented in the EMS of the islanded microgrid. An EMS control strategy and a droop based MPC control algorithm have also been proposed to sustain the normal operation of the islanded microgrid. Simulation studies have been conducted to analyze the operation of an islanded microgrid with the proposed control strategy. It has been shown that the proposed control strategy can successfully maintain the operation of a microgrid during islanded condition. 


\section{CHAPTER 5 CONTROL AND OPERATION OF A DC GRID BASED WIND POWER GENERATION SYSTEM IN A MICROGRID [133]}

\section{Nomenclature}

$\begin{array}{ll}P_{w t, o p t} & \text { Optimized wind power. } \\ k_{\text {opt }} & \text { Optimized constant. } \\ \omega_{r, \text { opt }} & \text { Wind turbine speed for optimized power generation. } \\ C_{p, o p t} & \text { Optimized power coefficient of the turbine. } \\ \rho & \text { Air density. } \\ A & \text { Area swept by the rotor blades. } \\ \lambda_{o p t} & \text { Optimized tip speed ratio. } \\ v & \text { Wind speed. } \\ R_{b} & \text { Radius of the blade. } \\ e_{s a}, e_{s b}, e_{s c} & \text { Balanced three-phase AC voltage source to model the PMSG. } \\ R_{s} & \text { Series resistance of the PMSG. } \\ L_{s} & \text { Series inductance of the PMSG. } \\ i_{s a}, i_{s b}, i_{s c} & \text { Three-phase output currents of the PMSG. } \\ V_{d c} & \text { DC output voltage of the rectifier. } \\ R & \text { Inverter loss. } \\ L_{f} \text { and } C_{f} & \text { Inductance and capacitance of the LC filter of the inverter. } \\ i_{D G} & \text { Inverter output current. } \\ i & \text { Current flowing through } L_{f} . \\ i_{C_{f}} & \text { Current flowing through } C_{f} . \\ v_{D G} & \text { Inverter output voltage. } \\ u & \text { Control signal for the inverter. } \\ T_{s} & \text { Sampling time to discretize inverter model. } \\ & \end{array}$

\subsection{Introduction}

Along with the discussion in the previous two chapters regarding the control of DG inverter operation in microgrid, it can be seen that centralized approach and 
decentralized approach are tightly connected for AC microgrids. Even though to achieve automation, decentralized strategy is preferred, a combination of centralized and decentralized approach is necessary to maintain the operation of a microgrid during both grid-connected and islanded conditions.

In this chapter, the study of control strategy of DG inverter operation extends to DC microgrids. As mentioned in Chapter 2, the government of Singapore is looking for possibilities to harness the excessive wind power generated by the cooling fans in poultry farms to reduce the farms' demand on the grid. An alternative solution using a DC grid based distribution network where the AC outputs of the WGs in a poultry farm are rectified to a common voltage at the DC grid is proposed in this research. The most significant advantage of the proposed system is that only the voltage at the DC grid has to be controlled for parallel operation of several WGs without the need to synchronize the voltage, frequency and phase, thus allowing the WGs to be turned on or off anytime without causing any disruptions.

\subsection{System Description and Modeling}

\subsubsection{System Description}

The overall configuration of the proposed DC grid based wind power generation system for the poultry farm is shown in Figure 5-1. The system can operate either connected to or islanded from the distribution grid and consists of four $10 \mathrm{~kW}$ permanent magnet synchronous generators (PMSGs) which are driven by the variable speed WTs. The PMSG is considered in this chapter because it does not require a DC excitation system that will increase the design complexity of the control hardware. The three-phase output of each PMSG is connected to a three-phase converter (i.e., converters $\mathrm{A}, \mathrm{B}, \mathrm{C}$ and $\mathrm{D}$ ), which operates as a rectifier to regulate the DC output voltage of each PMSG to the desired level at the DC grid. The aggregated power at the DC grid is inverted by two inverters (i.e., inverters 1 and 2) with each rated at $40 \mathrm{~kW}$. Instead of using individual inverter at the output of each WG, the use of two inverters between the DC grid and the AC grid is proposed. This architecture minimizes the 
need to synchronize the frequency, voltage and phase, reduces the need for multiple inverters at the generation side, and provides the flexibility for the plug and play connection of WGs to the DC grid. The availability of the DC grid will also enable the supply of power to DC loads more efficiently by reducing another AC/DC conversion.

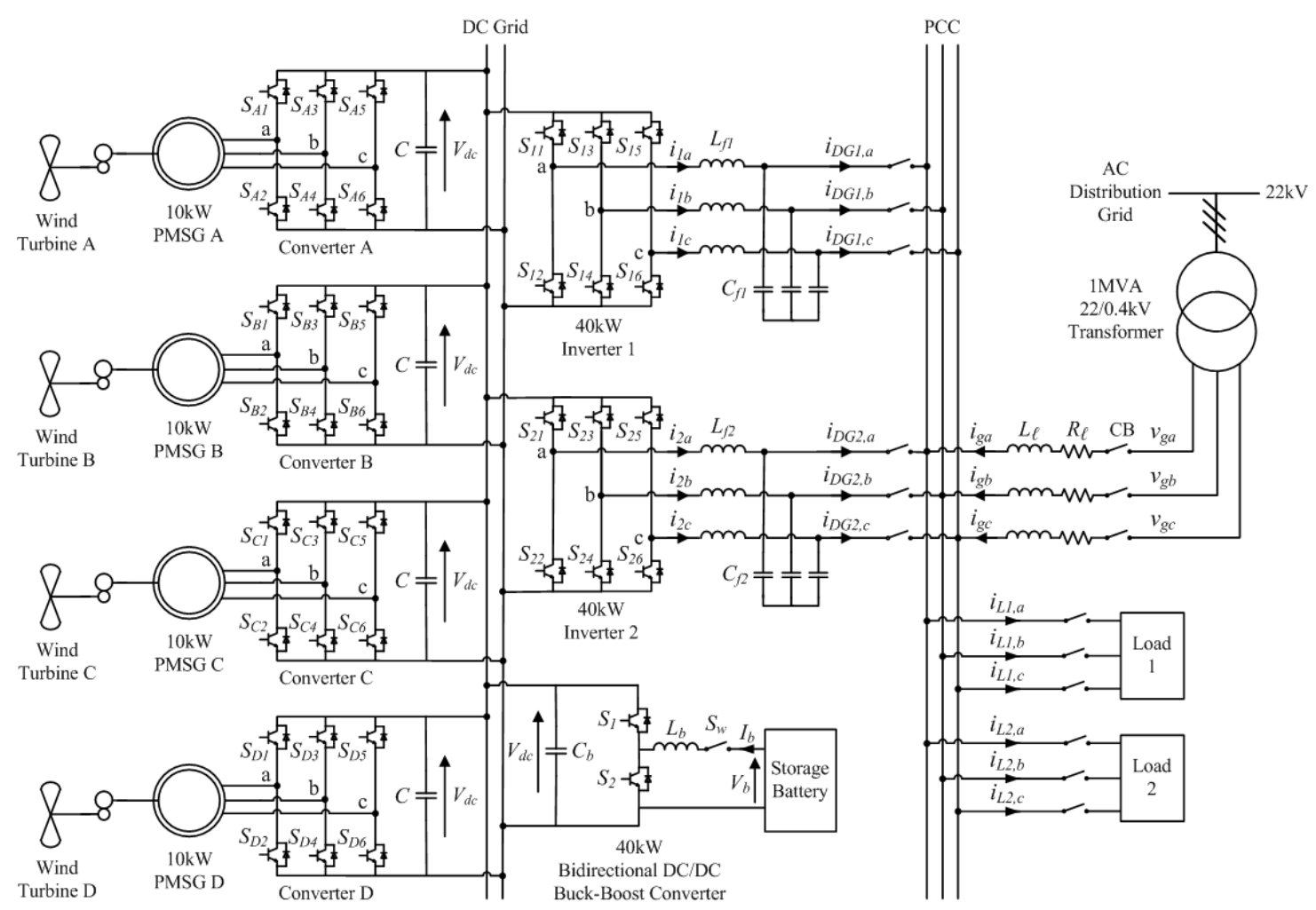

Figure 5-1: Overall configuration of the proposed DC grid based wind power generation system in a microgrid.

The coordination of the converters and inverters is achieved through a centralized EMS. The EMS controls and monitors the power dispatch by each wind generator and the load power consumption in the microgrid through a centralized server. To prevent excessive circulating currents between the inverters, the inverter output voltages of inverters 1 and 2 are regulated to the same voltage. Through the EMS, the output voltages of inverters 1 and 2 are continuously monitored to ensure that the inverters maintain the same output voltages. The centralized EMS is also responsible for other aspects of power management such as load forecasting, unit commitment, economic dispatch and optimum power flow. Important information such as field measurements from smart meters, transformer tap positions and $\mathrm{CB}$ status are all sent to the centralized server for processing through wireline/wireless communication. During 
normal operation, the two inverters will share the maximum output from the PMSGs (i.e., each inverter shares $20 \mathrm{~kW}$ ). The maximum power generated by each wind turbine is estimated from the optimal wind power $P_{w t, o p t}$ as follows [134]:

$$
\begin{gathered}
P_{w t, o p t}=k_{o p t}\left(\omega_{r, o p t}\right)^{3} \\
k_{o p t}=\frac{1}{2} C_{p, o p t} \rho A\left(\frac{R}{\lambda_{o p t}}\right)^{3} \\
\omega_{r, o p t}=\frac{\lambda_{o p t} v}{R_{b}}
\end{gathered}
$$

where $k_{\text {opt }}$ is the optimized constant, $\omega_{r, \text { opt }}$ is the wind turbine speed for optimum power generation, $C_{p, o p t}$ is the optimum power coefficient of the turbine, $\rho$ is the air density, $A$ is the area swept by the rotor blades, $\lambda_{\text {opt }}$ is the optimum tip speed ratio, $v$ is the wind speed and $R$ is the radius of the blade. When one inverter fails to operate or is under maintenance, the other inverter can handle the maximum power output of 40 $\mathrm{kW}$ from the PMSGs. Thus the proposed topology offers increased reliability and ensures continuous operation of the wind power generation system when either inverter 1 or inverter 2 is disconnected from operation. An $80 \mathrm{Ah} \mathrm{SB}$, which is sized according to [135], is connected to the DC grid through a $40 \mathrm{~kW}$ bidirectional DC/DC buck-boost converter to facilitate the charging and discharging operations when the microgrid operates connected to or islanded from the grid. The energy constraints of the SB in the proposed DC grid are determined based on the SOC limits given by

$$
\mathrm{SOC}_{\text {min }} \leq \mathrm{SOC} \leq \mathrm{SOC}_{\max }
$$

Although the SOC of the SB cannot be directly measured, it can be determined through the estimation methods as detailed in [113], [136]. With the use of a DC grid, the impact of fluctuations between power generation and demand can be reduced as the SB can swiftly come online to regulate the voltage at the DC grid. During off-peak periods when the electricity demand is low, the SB is charged up by the excess power 
generated by the WTs. Conversely, during peak periods when the electricity demand is high, the SB will supplement the generation of the WTs to the loads.

\subsubsection{System Operation}

When the microgrid is operating connected to the distribution grid, the WTs in the microgrid are responsible for providing local power support to the loads, thus reducing the burden of power delivered from the grid. The SB can be controlled to achieve different demand side management functions such as peak shaving and valley filling depending on the time-of-use (TOU) prices of electricity and SOC of the SB [9], [35], [137].

During islanded condition where the $\mathrm{CBs}$ disconnect the microgrid from the distribution grid, the WTs and the SB are only available sources to supply the load demand. The SB can supply for the deficit in real power to maintain the power balance of the microgrid as follows:

$$
P_{w t}+P_{s b}=P_{l o s s}+P_{l}
$$

where $P_{w t}$ is the real power generated by the WTs, $P_{s b}$ is the real power supplied by SB which is subjected to the constraint of the SB maximum power $P_{s b \text {, max }}$ that can be delivered during discharging and is given by

$$
P_{s b} \leq P_{s b, \max }
$$

$P_{l o s s}$ is the system loss, and $P_{l}$ is the real power that is supplied to the loads.

\subsubsection{AC/DC Rectifier Modeling}

Figure 5-2 shows the power circuit consisting of a PMSG which is connected to an AC/DC voltage source rectifier. The PMSG is modeled as a balanced three-phase AC voltage source $e_{s a}, e_{s b}, e_{s c}$ with series resistance $R_{s}$ and inductance $L_{s}$ [138], [139]. As 
shown in [129], the state equations for the PMSG currents $i_{s a}, i_{s b}, i_{s c}$ and the DC output voltage $V_{d c}$ of the converter can be expressed as follows:

$$
\begin{gathered}
L_{s} \frac{d i_{s}}{d t}=-R_{s} i_{s}+e_{s}-K S V_{d c} \\
C \frac{d V_{d c}}{d t}=i_{s}^{T} S-I_{d c}
\end{gathered}
$$

where $i_{s}=\left[\begin{array}{lll}i_{s a} & i_{s b} & i_{s c}\end{array}\right]^{T} ; e_{s}=\left[\begin{array}{lll}e_{s a} & e_{s b} & e_{s c}\end{array}\right]^{T} ; K=\left[\begin{array}{ccc}2 / 3 & -1 / 3 & -1 / 3 \\ -1 / 3 & 2 / 3 & -1 / 3 \\ -1 / 3 & -1 / 3 & 2 / 3\end{array}\right]$;

$S=\left[\begin{array}{lll}S_{a} & S_{b} & S_{c}\end{array}\right]^{T}$ is the AC/DC rectifier switching functions which are defined as

$$
S_{j}=\left\{\begin{array}{ll}
1, & S_{j} \text { is ON } \\
0, & S_{j} \text { is OFF }
\end{array} \quad \text { for } j=a, b, c\right.
$$

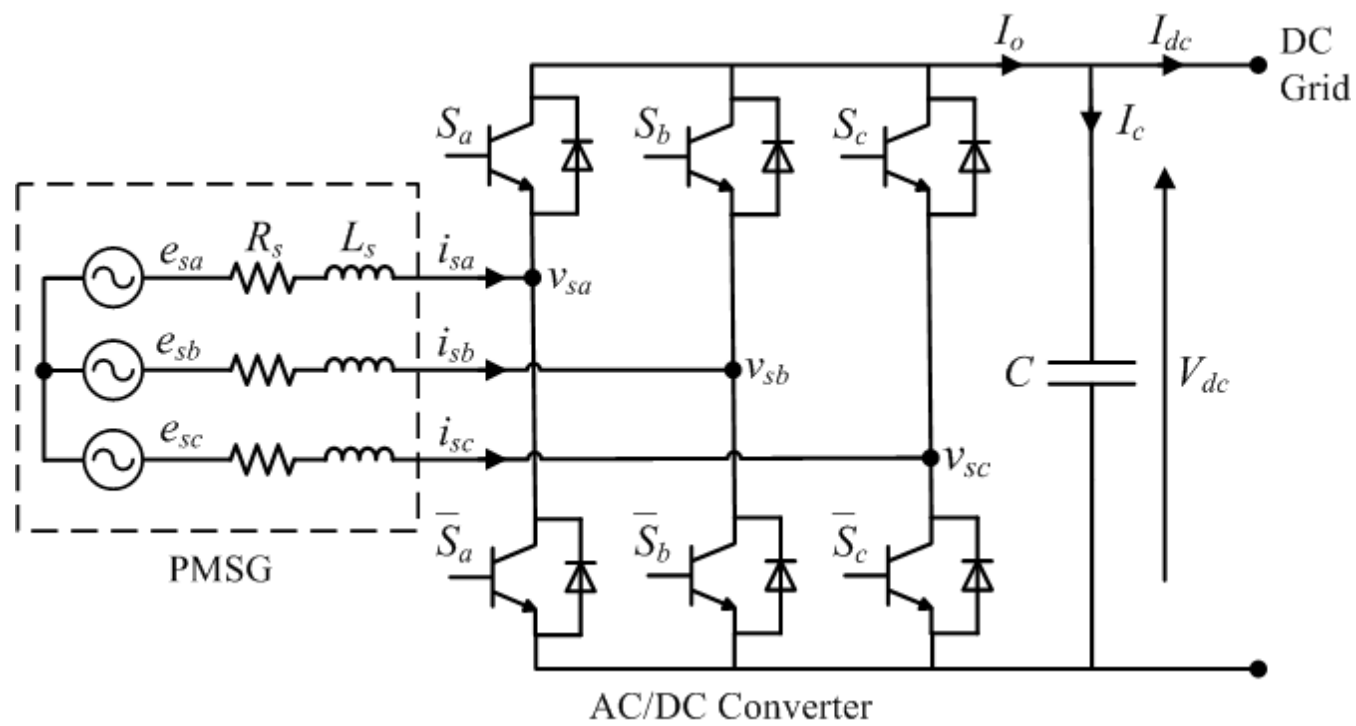

Figure 5-2: Power circuit of a PMSG connected to an AC/DC voltage source rectifier.

\subsubsection{DG Inverter Modeling}

The two 40kW three-phase DG inverters which connect the DC grid to the PCC are identical, and the single-phase representation of the three-phase DG inverter is shown in Figure 5-3. To derive a state-space model for the inverter, Kirchhoff's voltage and 
current laws are applied to loop $i$ and point $x$ respectively, and the following equations are obtained:

$$
\begin{gathered}
L_{f} \frac{d i}{d t}+i R+v_{D G}=u V_{d c} \\
i_{D G}=i-i_{C_{f}}
\end{gathered}
$$

where $V_{d c}$ is the DC grid voltage, $u$ is the control signal, $R$ is the inverter loss, $L_{f}$ and $C_{f}$ are the inductance and capacitance of the low-pass filter (LPF) respectively, $i_{D G}$ is the inverter output current, $i$ is the current flowing through $L_{f}, i_{C_{f}}$ is the current flowing through $C_{f}$, and $v_{D G}$ is the inverter output voltage.

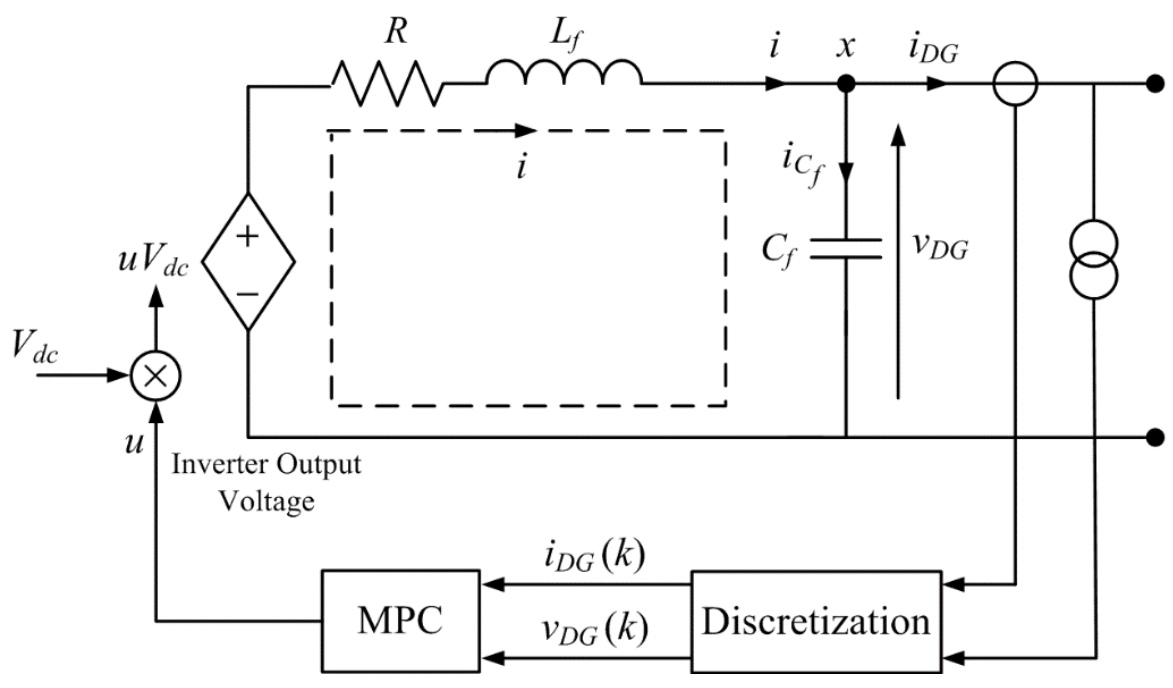

Figure 5-3: Single-phase representation of the three-phase DG inverter.

During grid-connected condition, the inverters are connected to the distribution grid and are operated in the CCM because the magnitude and the frequency of the output voltage are tied to the grid voltage. Thus, the discrete state-space equations for the inverter model operating in the CCM can be expressed with sampling time $T_{s}$ as follows:

$$
x_{g}(k+1)=A_{g} x_{g}(k)+B_{g 1} v_{g}(k)+B_{g 2} u_{g}(k)
$$




$$
y_{g}(k)=C_{g} x_{g}(k)+D_{g} v_{g}(k)
$$

where the subscript $g$ represents the inverter model during grid-connected condition, $k$ is the discretized present time step, and

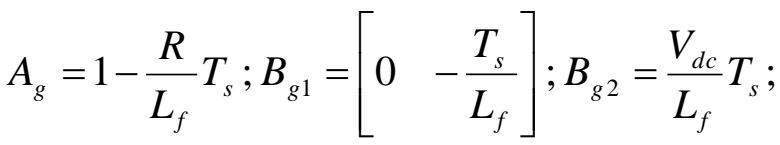

$$
\begin{aligned}
& C_{g}=1 ; D_{g}=\left[\begin{array}{ll}
\frac{C_{f}}{T_{s}} & -\frac{C_{f}}{T_{s}}
\end{array}\right] \text {; }
\end{aligned}
$$

$x_{g}(k)=i(k)$ is the state vector; $v_{g}(k)=\left[v_{D G}(k+1) \quad v_{D G}(k)\right]^{T}$ is the exogenous input; $u_{g}(k)$ is the control signal with $-1 \leq u_{g}(k) \leq 1$; and $y_{g}(k)=i_{D G}(k)$ is the output. The exogenous input of the inverter output voltage can be calculated using state estimation. In this chapter, the grid is set as a large power system, which means that the grid voltage is a stable three-phase sinusoidal voltage. Hence, when operating in the CCM, a three-phase sinusoidal signal can be directly used as the exogenous input.

During islanded condition, the inverters will be operated in the VCM. The voltage of the PCC will be maintained by the inverters when the microgrid is islanded from the grid. As compared to $T_{s}$, the rate of change of the inverter output current is much slower. Therefore, the following assumption is made when deriving the state-space equations for the inverter operating in the VCM [140]:

$$
\frac{d i_{D G}}{d t}=0
$$

Based on the above mentioned assumption, the discrete state-space equations of the inverter model operating in the VCM can be expressed as follows:

$$
\begin{gathered}
x_{i}(k+1)=A_{i} x_{i}(k)+B_{i} u_{i}(k) \\
y_{i}(k)=C_{i} x_{i}(k)
\end{gathered}
$$


where the subscript $i$ represents the model of the inverter during islanded condition and

$$
A_{i}=\left[\begin{array}{ccc}
1-\frac{R}{L_{f}} T_{s} & -\frac{T_{s}}{L_{f}} & 0 \\
\frac{T_{s}}{L_{f}} & 1 & -\frac{T_{s}}{C_{f}} \\
0 & 0 & 1
\end{array}\right] ; B_{i}=\left[\begin{array}{ccc}
\frac{V_{d c}}{L_{f}} T_{s} & 0 & 0
\end{array}\right] ; C_{i}=\left[\begin{array}{lll}
0 & 1 & 0
\end{array}\right] ;
$$

$x_{i}(k)=\left[i(k) \quad v_{D G}(k) \quad i_{D G}(k)\right]^{T}$ is the state vector; $u_{i}(k)$ is the control signal with $-1 \leq$ $u_{i}(k) \leq 1$; and $y_{i}(k)=v_{D G}(k)$ is the output. During islanded condition, the inverters are required to deliver all the available power from the PMSGs to the loads. Therefore, only the inverter output voltage is controlled and the output current is determined from the amount of available power.

\subsection{Control Design}

\subsubsection{Control Design for the AC/DC Rectifier}

Figure 5-4 shows the configuration of the proposed controller for each AC/DC voltage source rectifier which is employed to maintain the DC output voltage $V_{d c}$ of each converter and compensate for any variation in $V_{d c}$ due to any power imbalance in the DC grid. The power imbalance will induce a voltage error $\left(V_{d c}^{*}-V_{d c}\right)$ at the DC grid, which is then fed into a PI controller to generate a current reference $i_{d}^{*}$ for $i_{d}$ to track. To eliminate the presence of high frequency switching ripples at the DC grid, $V_{d c}$ is first passed through a first-order LPF. The current $i_{q}$ is controlled to be zero so that the PMSG only delivers real power. The current errors $\Delta i_{d}$ and $\Delta i_{q}$ are then converted into the $a b c$ frame and fed into a PR controller to generate the required control signals using PWM generator. 


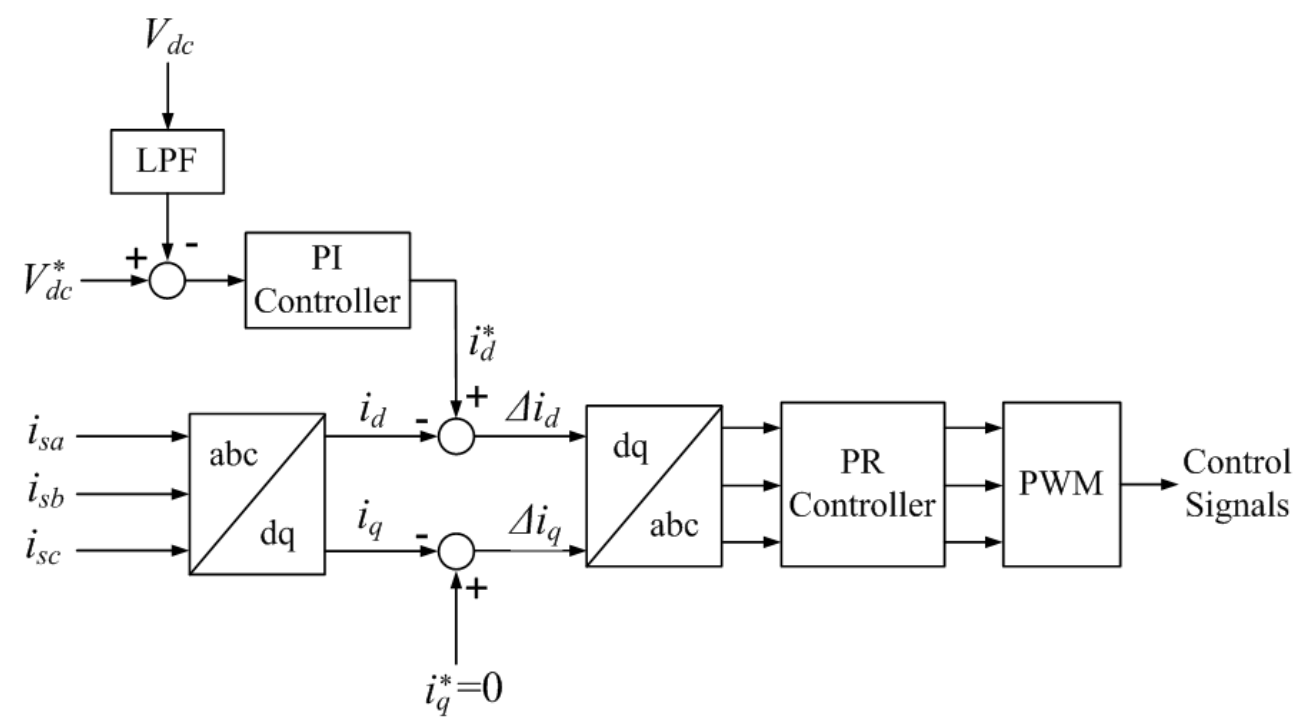

Figure 5-4: Configuration of the proposed controller for the AC/DC converter.

\subsubsection{Control Design for the DG Inverter}

In order for the microgrid to operate in both grid-connected and islanded modes of operation, a model-based controller using MPC is proposed for the control of the inverters. MPC is a model-based controller and adopts a receding horizon approach in which the optimization algorithm will compute a sequence of control actions to minimize the selected objectives for the whole control horizon, but only execute the first control action for the inverter. At the next time step, the optimization process is repeated based on new measurements over a shifted prediction horizon. By doing so, MPC can make the output track the reference at the next step, as well as plan and correct its control signals along the control process. This will guarantee a better transient response compared to conventional PID/PR controllers [129], [140]. To derive the control algorithm for the inverters, the state-space equations are transformed into augmented state-space equations by defining the incremental variables in the following format:

$$
\Delta \xi(k)=\xi(k)-\xi(k-1)
$$

where $\xi$ represents each variable in the inverter model, such as $v_{D G}, i_{D G}, i$ and $u$ as shown in Figure 5-3. 
By defining the incremental variables, the augmented state-space model for the inverter model operating in the CCM during grid-connected condition can be expressed as follows:

$$
\begin{gathered}
X_{g}(k+1)=A_{g_{-} a u g} X_{g}(k)+B_{g_{1} \_a u g} V_{g}(k)+B_{g 2_{-} a u g} U_{g}(k) \\
Y_{g}(k)=C_{g_{-} a u g} X_{g}(k)
\end{gathered}
$$

where

$A_{g_{-} a u g}=\left[\begin{array}{cc}1-\frac{R}{L_{f}} T_{s} & 0 \\ 1-\frac{R}{L_{f}} T_{s} & 1\end{array}\right] ; B_{g 1_{-} a u g}=\left[\begin{array}{ccc}0 & 0 & -\frac{T_{s}}{L_{f}} \\ -\frac{C_{f}}{T_{s}} & \frac{C_{f}}{T_{s}} & -\frac{T_{s}}{L_{f}}\end{array}\right] ; B_{g 2_{-} a u g}=\left[\begin{array}{cc}\frac{V_{d c}}{L_{f}} T_{s} & -\frac{V_{d c}}{L_{f}} T_{s} \\ \frac{V_{d c}}{L_{f}} T_{s} & -\frac{V_{d c}}{L_{f}} T_{s}\end{array}\right] ;$ $C_{g_{-} \text {aug }}=\left[\begin{array}{ll}0 & 1\end{array}\right] \quad ; \quad X_{g}(k)=\left[\Delta i(k) \quad i_{D G}(k)\right]^{T} \quad$ is $\quad$ the state vector; $V_{g}(k)=\left[\Delta v_{D G}(k+2) \quad \Delta v_{D G}(k+1) \quad \Delta v_{D G}(k)\right]^{T}$ is the exogenous input; $U_{g}(k)=\Delta u_{g}(k)$ is the control signal; and $Y_{g}(k)=i_{D G}(k)$ is the output.

Similarly, the augmented state-space model for the inverter model operating in the VCM during islanded condition can be expressed as follows:

$$
\begin{gathered}
X_{i}(k+1)=A_{i_{-} a u g} X_{i}(k)+B_{i_{-} \text {aug }} U_{i}(k) \\
Y_{i}(k)=C_{i_{-} a u g} X_{i}(k)
\end{gathered}
$$

where

$$
A_{i_{-} \text {aug }}=\left[\begin{array}{cccc}
1-\frac{R}{L_{f}} T_{s} & -\frac{T_{s}}{L_{f}} & 0 & 0 \\
\frac{T_{s}}{L_{f}} & 1 & -\frac{T_{s}}{L_{f}} & 0 \\
0 & 0 & 1 & 0 \\
\frac{T_{s}}{L_{f}} & 1 & \frac{T_{s}}{L_{f}} & 1
\end{array}\right] ; B_{i_{-} \text {aug }}=\left[\begin{array}{c}
\frac{V_{d c}}{L_{f}} T_{s} \\
0 \\
0 \\
0
\end{array}\right] ; C_{i_{-} \text {aug }}=\left[\begin{array}{llll}
0 & 0 & 0 & 1
\end{array}\right] ;
$$

$X_{i}(k)=\left[\begin{array}{llll}\Delta i(k) & \Delta v_{D G}(k) & \Delta i_{D G}(k) & v_{D G}(k)\end{array}\right]^{T}$ is the state vector; $U_{i}(k)=\Delta u_{i}(k)$ is the control signal; and $Y_{i}(k)=v_{D G}(k)$ is the output. 
For the control of the two augmented models in the CCM and the VCM, the following cost function is solved using quadratic programming in the proposed MPC algorithm [140]:

$$
J=\left(R_{s}-Y_{j}\right)^{T}\left(R_{s}-Y_{j}\right)+U_{j}^{T} Q U_{j}
$$

subject to the constraint

$$
-1 \leq u_{j}(k) \leq 1
$$

where $R_{S}$ is the set-point matrix, $Q$ is the tuning matrix for the desired closed-loop performance, $Y_{j}$ is the output of either the augmented model in the CCM or VCM (i.e., $Y_{g}$ or $\left.Y_{i}\right), U_{j}$ is the control signal of either the augmented model in the CCM or VCM (i.e., $U_{g}$ or $U_{i}$ ). The first part of the cost function is to compare the output of the augmented model $Y_{j}$ with the reference $R_{s}$ and to ensure that the output tracks the reference with minimum error. The second part of the cost function is to calculate the weighted factor of the control signal and to ensure that the control signal generated by the MPC algorithm is within the constraints. The quadratic programming will ensure that the optimal solution for the control signal deviation $\Delta u$ is achieved while minimizing the cost function $J$. After the control signal $u$ is generated by the MPC algorithm, it will be applied to the DG inverter as shown in Figure 5-3.

\subsection{Numerical Simulation Analysis}

The simulation model of the proposed DC grid based wind power generation system shown in Figure 5-1 is implemented in MATLAB/Simulink. The effectiveness of the proposed design concept is evaluated under different operating conditions when the microgrid is operating in the grid-connected or islanded mode of operation. The system parameters are given in Table 5-1. The impedances of the distribution line are obtained from [121]. In practical implementations, the values of the converter and inverter loss resistance are not precisely known. Therefore, these values have been coarsely estimated. 
Table 5-1: Parameters of the Proposed System

\begin{tabular}{ll}
\hline \hline Parameter & Value \\
\hline Distribution grid voltage & $v_{g}=230 \mathrm{~V}$ (phase) \\
DC grid voltage & $V_{d c}=500 \mathrm{~V}$ \\
PMSG stator impedance & $R_{S}=0.2 \Omega, L_{s}=2.4 \mathrm{mH}$ \\
Distribution line impedance & $R_{\ell}=7.5 \mathrm{~m} \Omega, L_{\ell}=25.7 \mu \mathrm{H}$ \\
Inverter LC filter & $L_{f}=1.2 \mathrm{mH}, C_{f}=20 \mu \mathrm{F}$ \\
Converter capacitor & $C=300 \mu \mathrm{F}$ \\
Converter and inverter loss resistance & $R=1 \mathrm{~m} \Omega$ \\
Load 1 rating & $P_{L 1}=35 \mathrm{~kW}, Q_{L 1}=8 \mathrm{kVAr}$ \\
Load 2 rating & $P_{L 2}=25 \mathrm{~kW}, Q_{L 2}=4 \mathrm{kVAr}$ \\
\hline
\end{tabular}

\subsubsection{Test Case 1: Failure of One Inverter During Grid-Connected Condition}

When the microgrid is operating in the grid-connected condition of operation, the proposed wind power generation system will supply power to meet part of the load demand. Under normal operating condition, the total power generated by the PMSGs at the DC grid is converted by inverters 1 and 2 which will share the total power supplied to the loads. When one of the inverters fails to operate and needs to be disconnected from the DC grid, the other inverter is required to handle all the power generated by the PMSGs. In this test case, an analysis on the microgrid operation when one of the inverters is disconnected from operation is conducted.

With each PMSG generating about $5.5 \mathrm{~kW}$ of real power, the total power generated by the four PMSGs is about $22 \mathrm{~kW}$ which is converted by inverters 1 and 2 into $20 \mathrm{~kW}$ and $8 \mathrm{kVAr}$ of real and reactive power respectively. Figures 5-5 and 5-6 show the waveforms of the real and reactive power delivered by inverters 1 and 2 for $0 \leq t<0.4 \mathrm{~s}$ respectively. For $0 \leq t<0.2 \mathrm{~s}$, both inverters 1 and 2 are in operation and each inverter delivers about $10 \mathrm{~kW}$ of real power and $4 \mathrm{kVAr}$ of reactive power to the loads. The remaining real and reactive power that is demanded by the loads is supplied by the grid which is shown in Figure 5-7. It can be seen from Figure 5-7 that the grid delivers $40 \mathrm{~kW}$ of real power and $4 \mathrm{kVAr}$ of reactive power to the loads for $0 \leq t<0.2 \mathrm{~s}$. The 
total real and reactive power supplied to the loads is about $60 \mathrm{~kW}$ and $12 \mathrm{kVAr}$ as shown in the power waveforms of Figure 5-8. The unsteady measurements observed in the power waveforms for $0 \leq t<0.08 \mathrm{~s}$ are because the controller requires a period of about 4 cycles to track the power references during the initialization period. As compared to conventional control strategies, it can be observed that the proposed MPC algorithm is able to quickly track and settle to the power reference. This is attributed to the optimization of the inverters through the model-based MPC control. Essentially, model-based control schemes are able to take into account the system parameters such that the overall performance can be optimized.
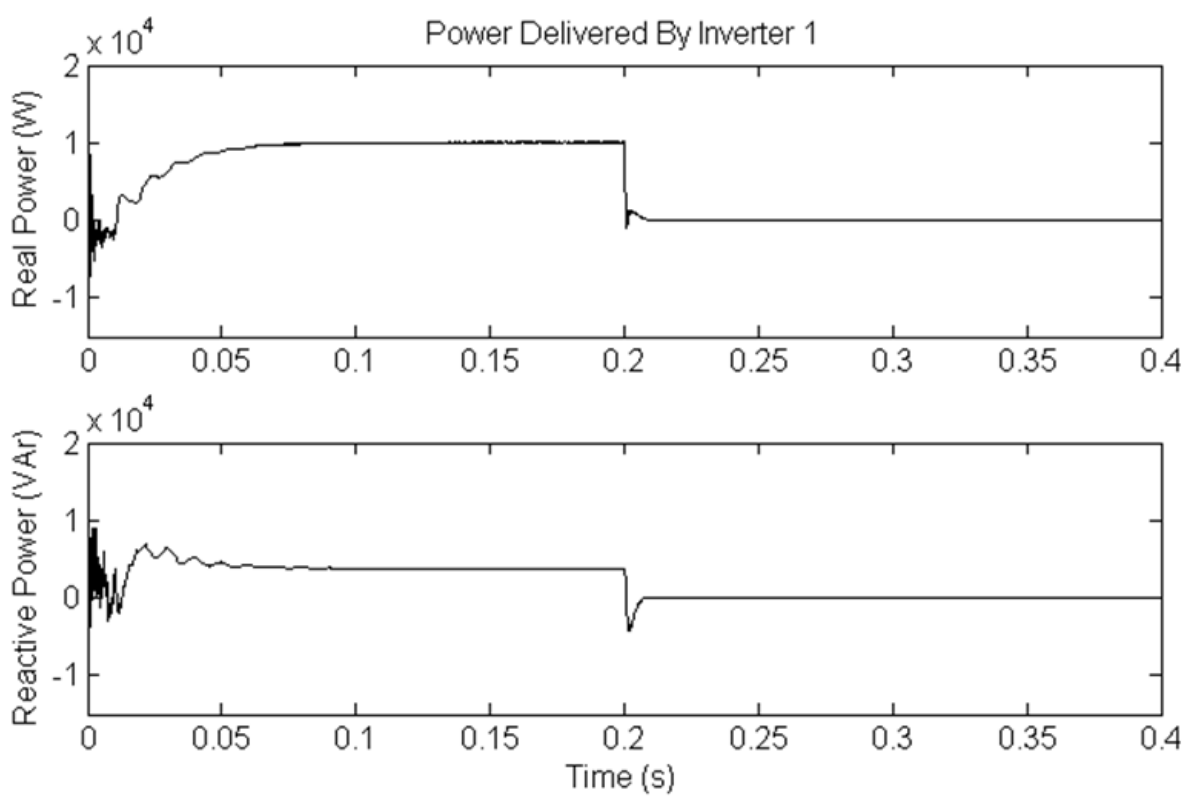

Figure 5-5: Real (top) and reactive (bottom) power delivered by inverter 1. 

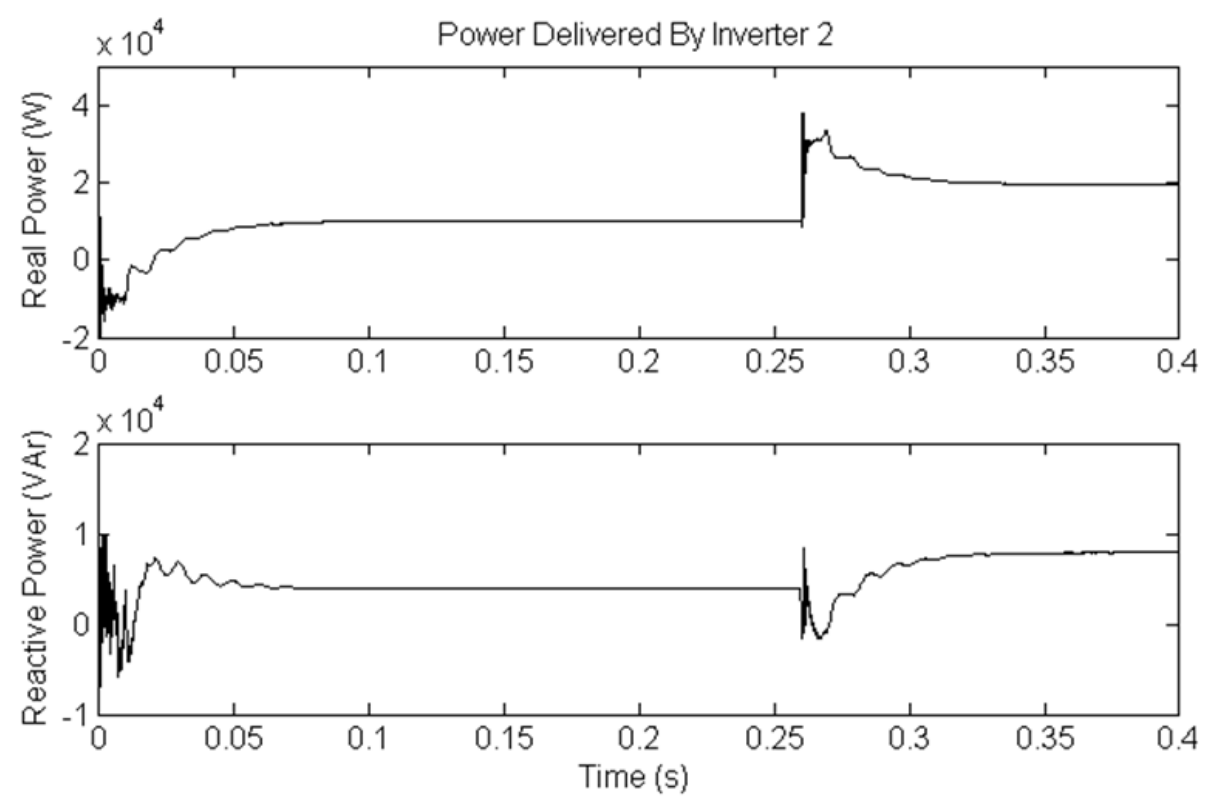

Figure 5-6: Real (top) and reactive (bottom) power delivered by inverter 2 .

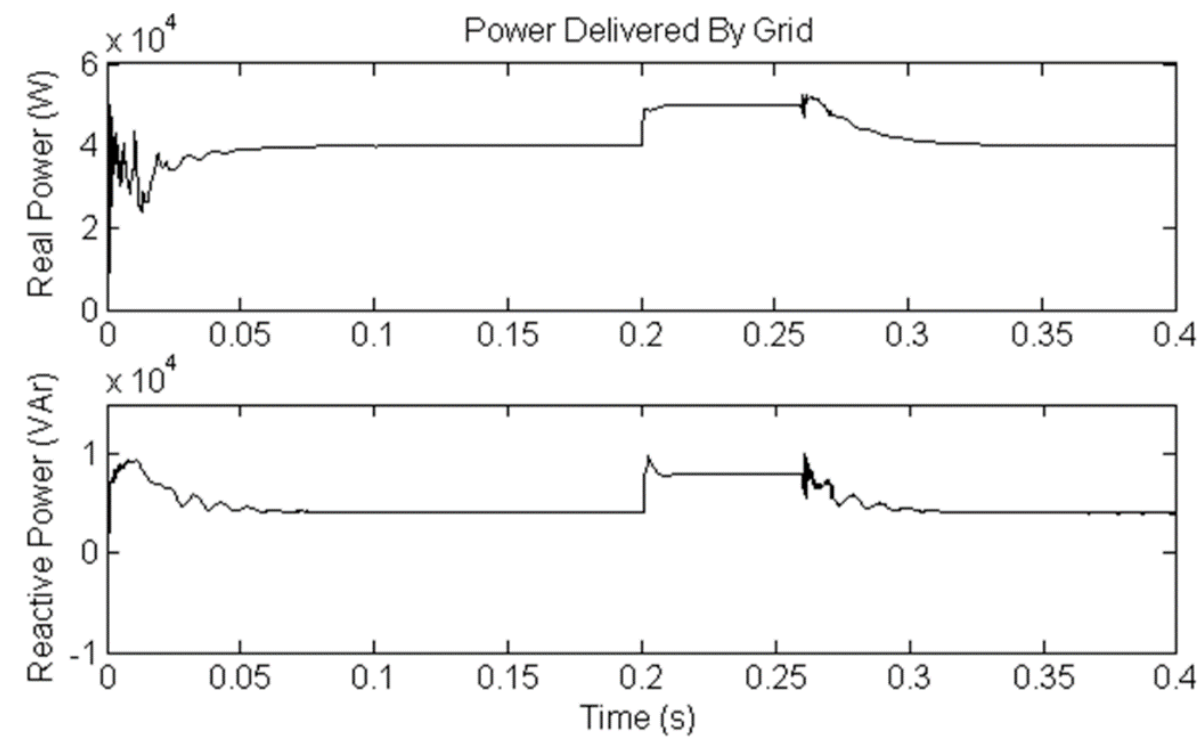

Figure 5-7: Real (top) and reactive (bottom) power delivered by the grid. 

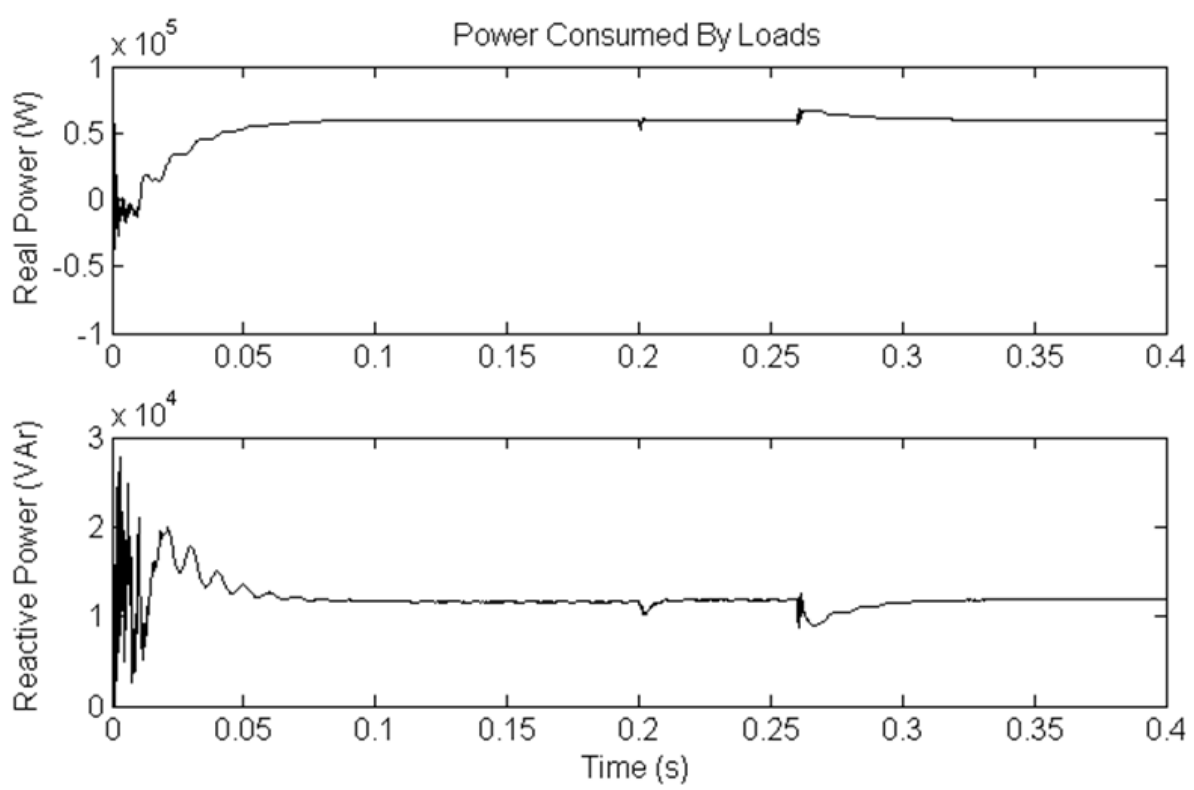

Figure 5-8: Real (top) and reactive (bottom) power consumed by the loads.

At $t=0.2 \mathrm{~s}$, inverter 1 fails to operate and is disconnected from the microgrid, resulting in a loss of $10 \mathrm{~kW}$ of real power and $4 \mathrm{kVAr}$ of reactive power supplied to the loads. As shown in Figure 5-5, the real and reactive power supplied by inverter 1 is decreased to zero in about half a cycle after inverter 1 is disconnected. This undelivered power causes a sudden power surge in the DC grid which corresponds to a voltage rise at $t=0.2 \mathrm{~s}$ as shown in Figure 5-9. To ensure that the load demand is met, the grid automatically increases its real and reactive power generation to $50 \mathrm{~kW}$ and 8 $\mathrm{kVAr}$ respectively at $t=0.2 \mathrm{~s}$, as shown in Figure 5-7. At $t=0.26 \mathrm{~s}$, the EMS of the microgrid increases the reference real and reactive power supplied by inverter 2 to 20 $\mathrm{kW}$ and $8 \mathrm{kVAr}$ respectively. A delay of 3 cycles is introduced to cater for the response time of the EMS to the loss of inverter 1. As shown in Figure 5-6, inverter 2 manages to increase its real and reactive power supplied to the loads to $20 \mathrm{~kW}$ and 8 $\mathrm{kVAr}$ for $0.26 \leq t<0.4 \mathrm{~s}$. At the same time, the grid decreases its real and reactive power back to $40 \mathrm{~kW}$ and $4 \mathrm{kVAr}$ as shown in Figure 5-7 respectively. The power balance in the microgrid is restored after 3 cycles from $t=0.26 \mathrm{~s}$. It is observed from Figure 5-9 that the voltage at the DC grid corresponds to a voltage dip at $t=0.26 \mathrm{~s}$ due to the increase in power drawn by inverter 2 and then returns to its nominal value of $500 \mathrm{~V}$ for $0.26 \leq t<0.4 \mathrm{~s}$. As observed in Figure 5-8, at $t=0.26 \mathrm{~s}$, the changes in power delivered by inverter 2 and the grid also cause a transient in the load power. 


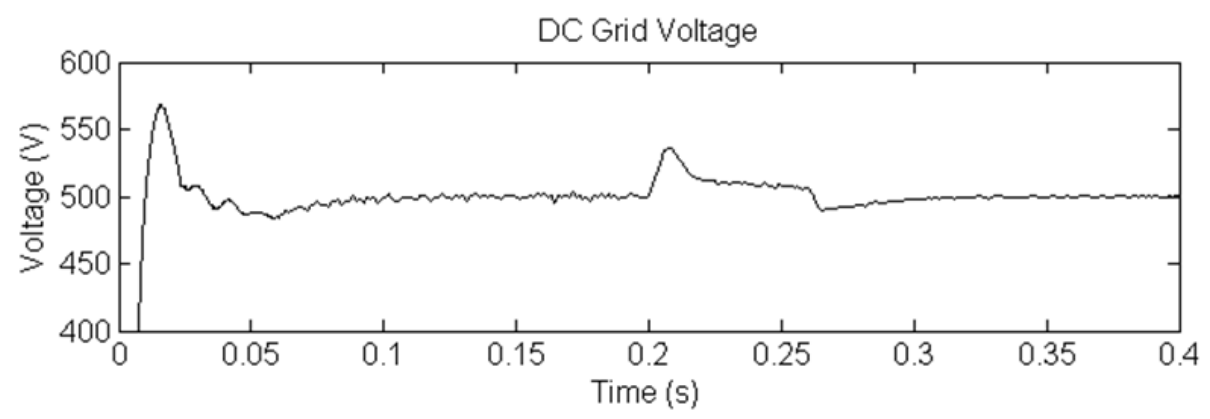

Figure 5-9: DC grid voltage.

\subsubsection{Test Case 2: Connection of AC/DC Rectifier during Grid-Connected Condition}

The most significant advantage of the proposed DC grid based wind power generation system is that it facilitates the connection of any PMSGs to the microgrid without the need to synchronize their voltage and frequency. This capability is demonstrated in this case study.

The microgrid operates connected to the grid and PMSG A is disconnected from the DC grid for $0 \leq t<0.2 \mathrm{~s}$ as shown in Figure 5-1. The real power generated from each of the remaining three PMSGs is maintained at $5.5 \mathrm{~kW}$ and their aggregated real power of $16.5 \mathrm{~kW}$ at the DC grid is converted by inverters 1 and 2 into $14 \mathrm{~kW}$ of real power and $8 \mathrm{kVAr}$ of reactive power. As shown in Figures 5-10 and 5-11, each inverter delivers real and reactive power of $7 \mathrm{~kW}$ and $4 \mathrm{kVAr}$ to the loads respectively. The rest of the real and reactive power demand of the loads is supplied by the grid as shown in Figure 5-12. It can be seen from Figure 5-12 that the grid delivers $46 \mathrm{~kW}$ of real power and $4 \mathrm{kVAr}$ of reactive power to the loads. 

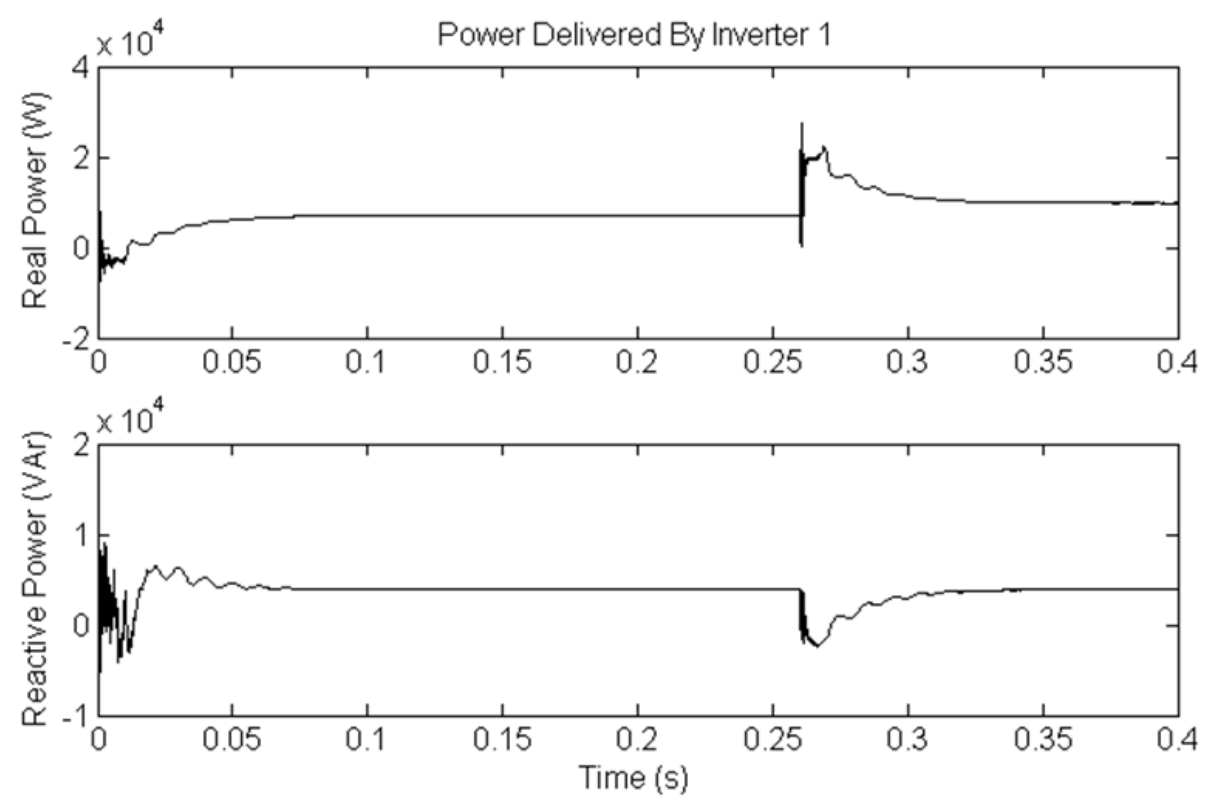

Figure 5-10: Real (top) and reactive (bottom) power delivered by inverter 1.
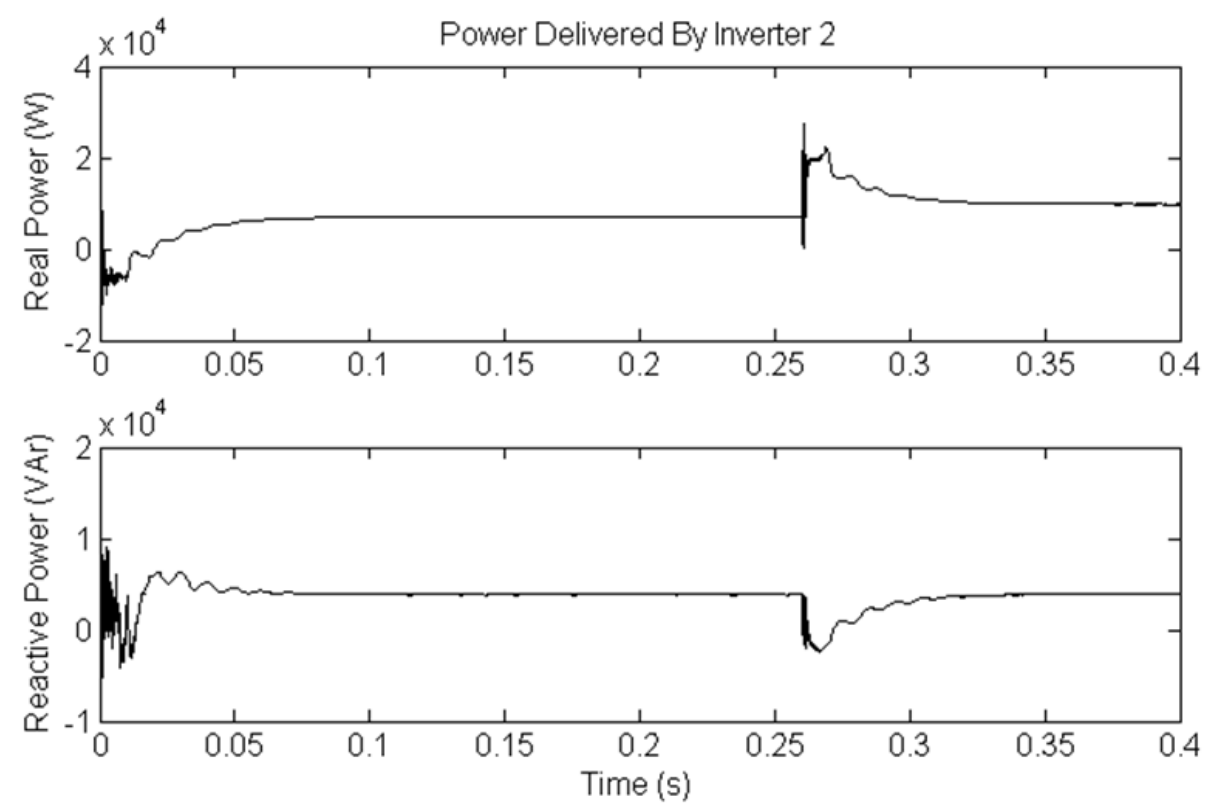

Figure 5-11: Real (top) and reactive (bottom) power delivered by inverter 2 . 

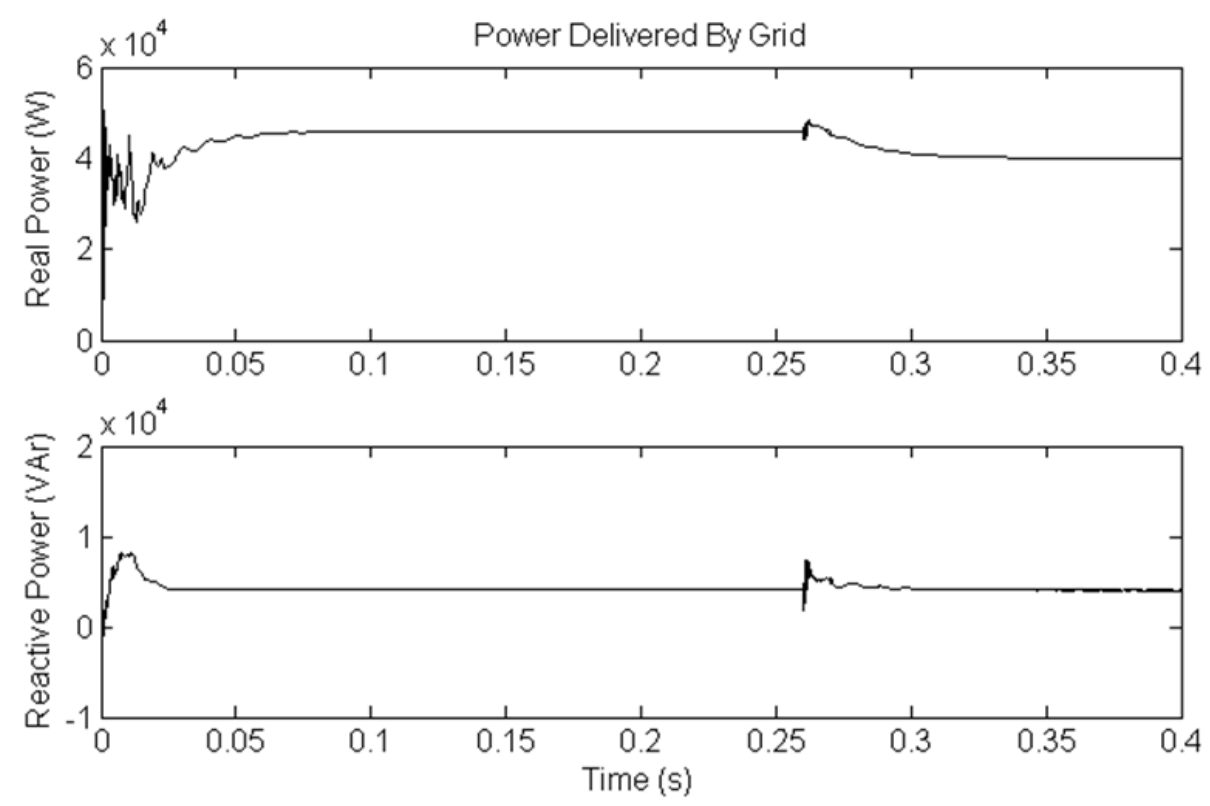

Figure 5-12: Real (top) and reactive (bottom) power delivered by the grid.

At $t=0.2 \mathrm{~s}$, PMSG A which generates real power of $5.5 \mathrm{~kW}$ is connected to the DC grid. This causes a sudden power surge at the DC grid and results in a voltage rise at $t=0.2 \mathrm{~s}$ as shown in the voltage waveform of Figure $5-13$. At $t=0.26 \mathrm{~s}$, the EMS increases the real power delivered by each inverter to $10 \mathrm{~kW}$ while the reactive power supplied by each inverter remains unchanged at $4 \mathrm{kVAr}$ as shown in Figures 5-10 and $5-11$. This causes a momentarily dip in the DC grid voltage at $t=0.26 \mathrm{~s}$ as observed in Figure 5-13 which is then restored back to its nominal voltage of $500 \mathrm{~V}$ for $0.26 \leq t<0.4$ s. The grid also simultaneously decreases its supply to $40 \mathrm{~kW}$ of real power for $0.26 \leq t<0.4 \mathrm{~s}$ while its reactive power remains constant at $4 \mathrm{kVAr}$ as shown in Figure $5-12$.

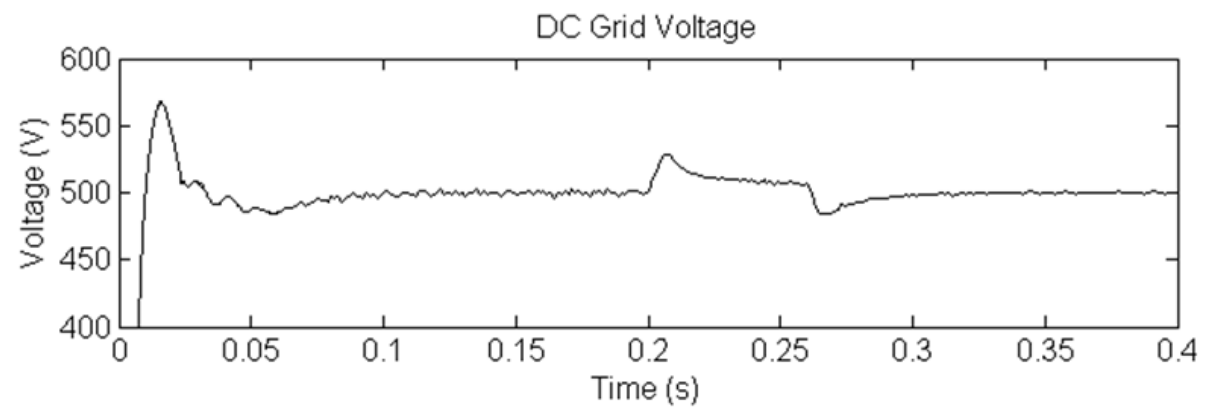

Figure 5-13: DC grid voltage. 


\subsubsection{Test Case 3: Islanded condition}

When the microgrid operates islanded from the distribution grid, the total generation from the PMSGs will be insufficient to supply for all the load demand. Under this condition, the SB is required to dispatch the necessary power to ensure that the microgrid continues to operate stably. The third case study shows the microgrid operation when it islands from the grid.

The microgrid is initially operating in the grid-connected condition. The grid is supplying real power of $40 \mathrm{~kW}$ and reactive power of $4 \mathrm{kVAr}$ to the loads for $0 \leq t<0.2$ $\mathrm{S}$ as shown in Figure 5-14 while each inverter is delivering real power of $10 \mathrm{~kW}$ and reactive power of $4 \mathrm{kVAr}$ to the loads as shown in Figures 5-15 and 5-16. At $t=0.2 \mathrm{~s}$, the microgrid is disconnected from the distribution grid by the CBs due to a fault occurring in the upstream network of the distribution grid. It can be seen from Figure 5-14 that the CBs fully separate the microgrid from the grid in about half a cycle, resulting in zero real and reactive power supplied by the grid for $0.2 \leq t<0.4 \mathrm{~s}$. With the loss of power supply from the grid, the power imbalance between the generation and load demand is detected by the EMS. To maintain the stability of the microgrid, the SB is tasked by the EMS to supply real power of $40 \mathrm{~kW}$ at $t=0.26 \mathrm{~s}$ as shown in Figure 5-17. At the same time, the real and reactive power delivered by each inverter is also increased by the EMS to $30 \mathrm{~kW}$ and $6 \mathrm{kVAr}$ as shown in Figures 5-15 and 5-16 respectively. Figure 5-18 shows the DC grid voltage where slight voltage fluctuations are observed at $t=0.26 \mathrm{~s}$. The initial voltage rise at $t=0.26 \mathrm{~s}$ is due to the power supplied by the SB while the subsequent voltage dip is due to the increase in power drawn by the inverters. 

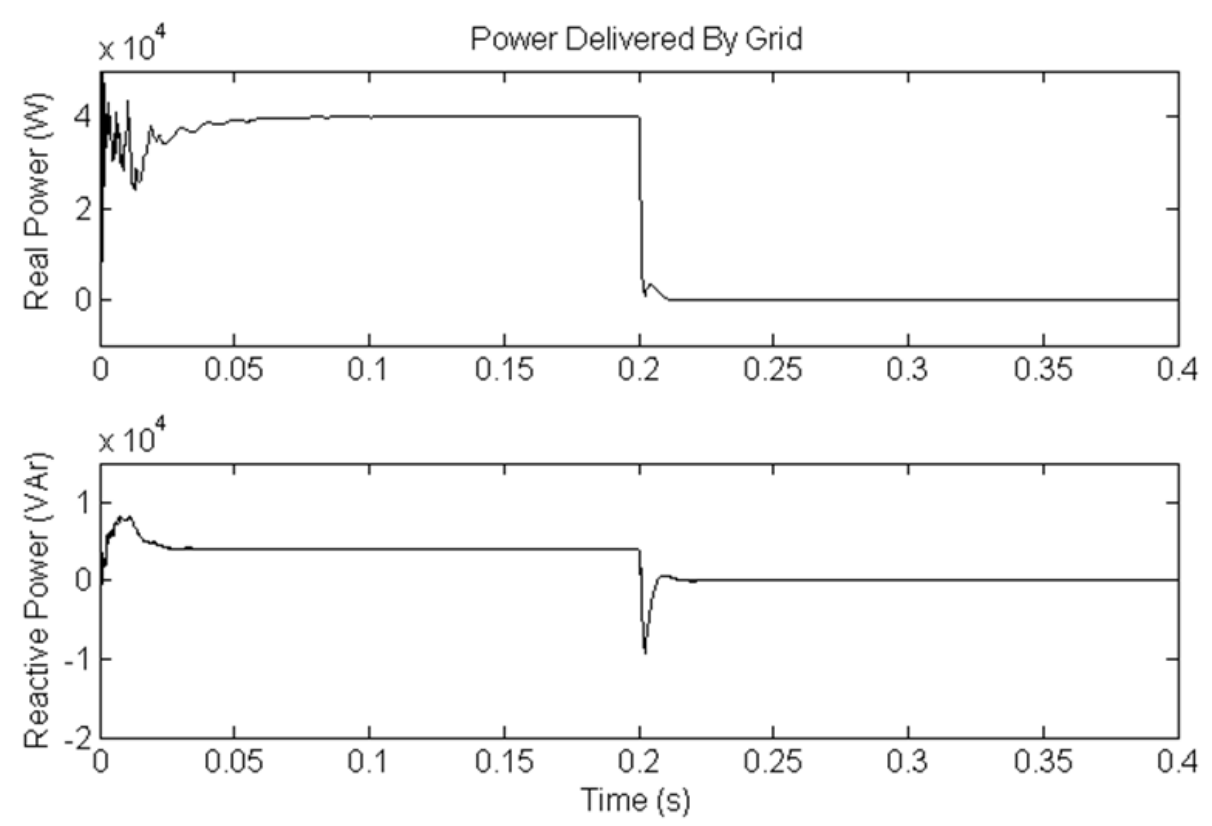

Figure 5-14: Real (top) and reactive (bottom) power delivered by the grid.
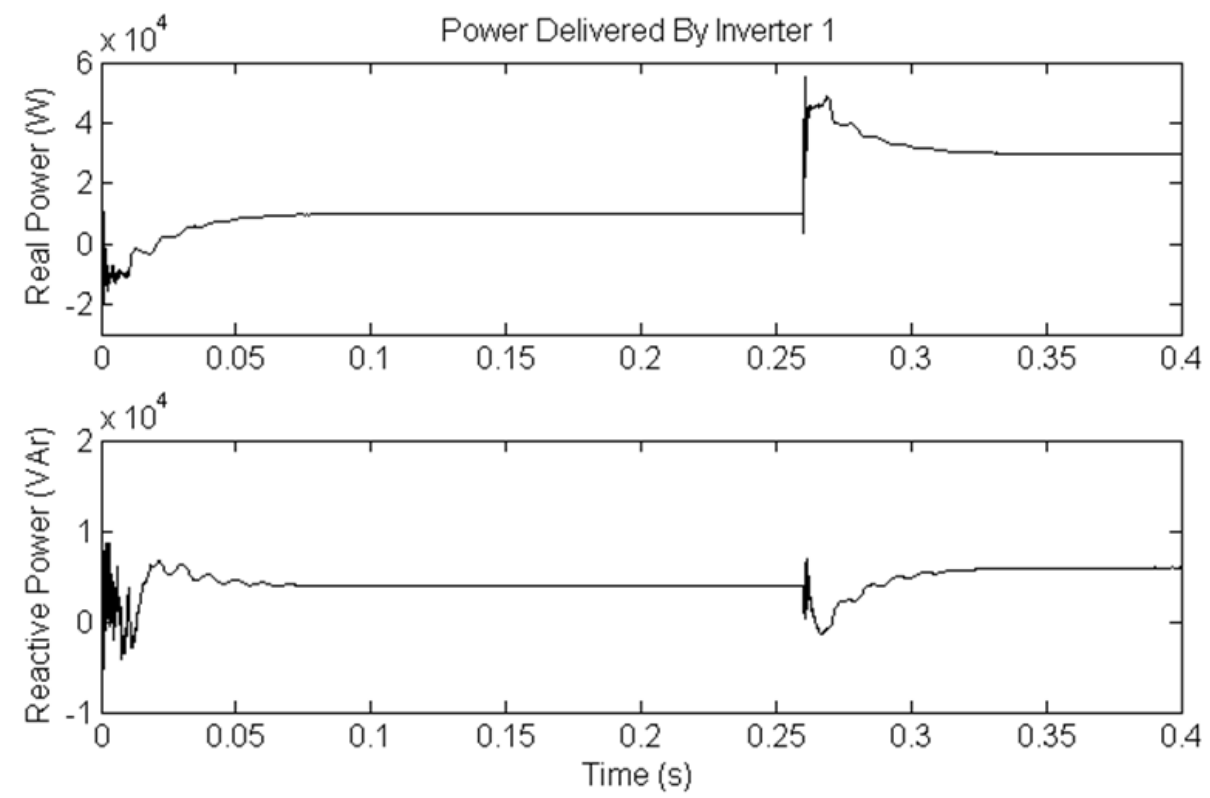

Figure 5-15: Real (top) and reactive (bottom) power delivered by inverter 1. 

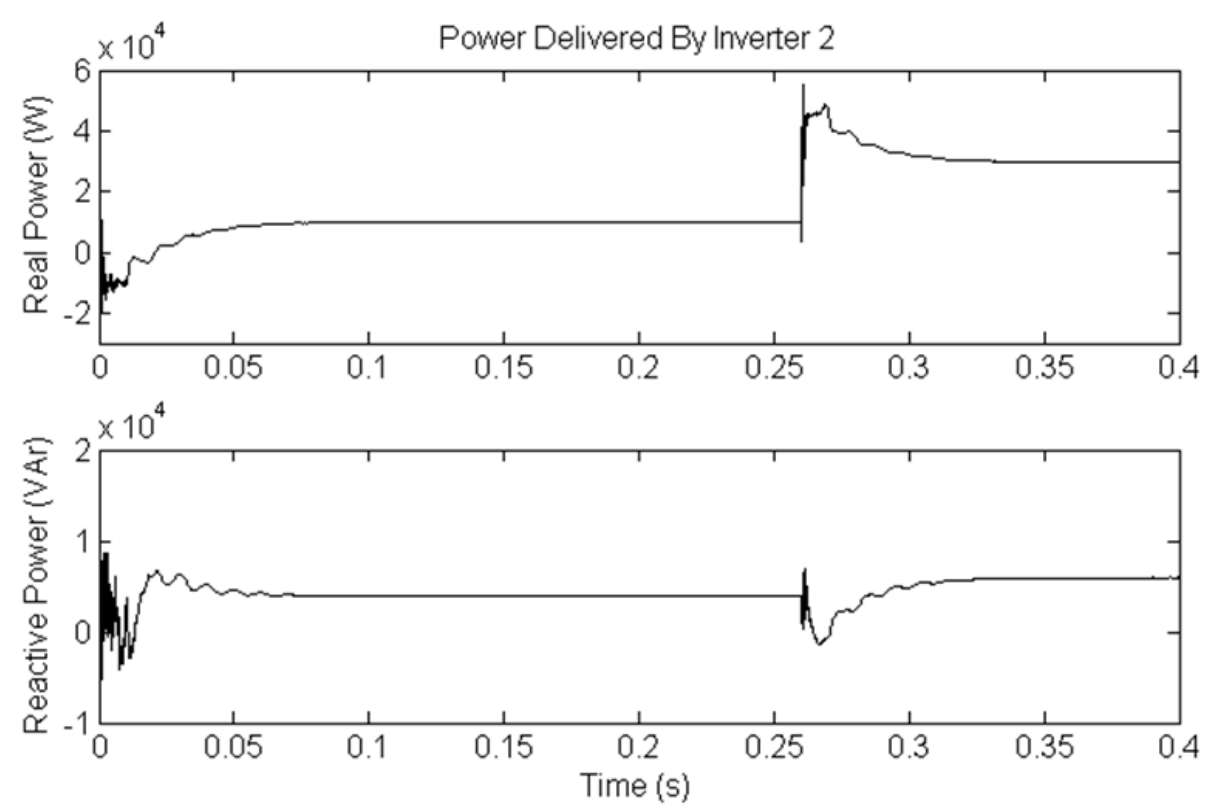

Figure 5-16: Real (top) and reactive (bottom) power delivered by inverter 2.

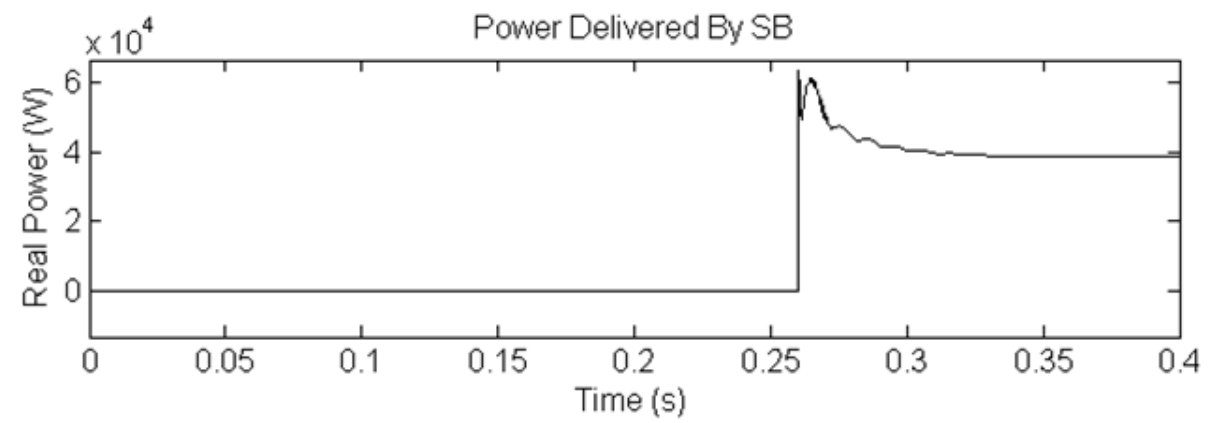

Figure 5-17: Real power delivered by SB.

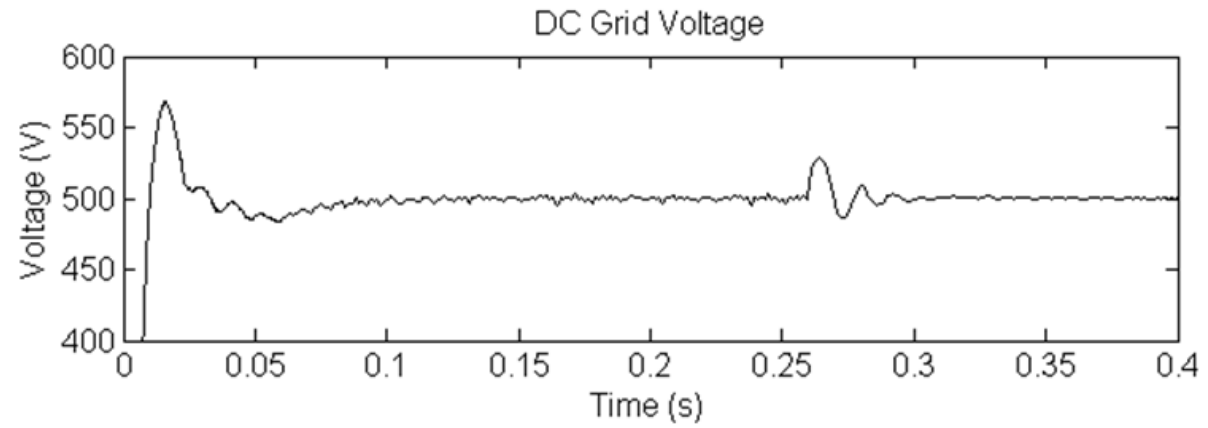

Figure 5-18: DC grid voltage. 


\subsection{Concluding Remarks}

In this chapter, the design of a DC grid based wind power generation system in a microgrid that enables parallel operation of several wind generators in a poultry farm has been presented. As compared to conventional wind power generation systems, the proposed microgrid architecture eliminates the need for voltage and frequency synchronization, thus allowing the wind generators to be switched on or off with minimal disturbances to the microgrid operation. The design concept has been verified through various test scenarios to demonstrate the operational capability of the proposed microgrid and the simulation results has shown that the proposed design concept is able to offer increased flexibility and reliability to the operation of the microgrid. However, the proposed control design still requires further experimental validation because measurement errors due to inaccuracies of the voltage and current sensors, and modeling errors due to variations in actual system parameters such as distribution line and transformer impedances will affect the performance of the controller in practical implementation. In addition, MPC relies on the accuracy of model establishment, hence further research on improving the controller robustness to modeling inaccuracy is required. The simulation results obtained and the analysis performed in this chapter serve as a basis for the design of a DC grid based wind power generation system in a microgrid. 


\section{CHAPTER 6 CONCLUSION AND RECOMMENDATIONS}

\subsection{Conclusions}

As more and more concerns are being raised because of the energy problem the world is facing, the integration of RESs and other kinds of DG units is critical, and it is becoming more and more common in different areas within different projects. Recognition of the microgrid concept has reached a new level worldwide. Demonstration projects and dedicated research works have investigated the different aspects of using AC and DC microgrids to integrate different DG units. To sustain the operation and improve the performance of different microgrids, various control strategies have been proposed. This is especially the case for DG inverters, which are one of the most common components in microgrids that are used to connect different DG units to the distribution network.

Centralized and decentralized approaches are used as ways to control inverter operations in microgrids, and their performances to maintain the operation of microgrids in different situations are analyzed. A centralized control strategy can control the operation of DG inverters in a more optimized manner. Various advanced algorithms can be used to perform optimization in a centralized way to control the operation of DG inverters. This results in an optimal operation in the microgrid, but it also places a heavy burden and a large responsibility on the communication network. To achieve autonomy, decentralized control strategy is used to control the operation of DG inverters in a localized manner. Usually, modified versions of traditional PI/PR control algorithms are used to enable the DG inverters to generate their own control signals within the local inverter controllers. The biggest challenge of decentralized control strategy is that microgrid will have low or no mechanical inertia during islanded condition when decentralized control strategy usually relies on local measurements, such as frequency, voltage, or power. To solve this problem, some research works are proposed using centralized control strategies or decentralized control strategies. In general, centralized and decentralized control strategies have their own advantages and disadvantages. This makes this topic interesting and worth investigating. 
In addition to different approaches used to control the operation of DG inverters, special attention is also given to the different operating conditions of the microgrid, i.e., grid-connected and islanded conditions. During both operating conditions, the operation of the microgrid needs to be sustained by controlling the operation of the DG inverters. During grid-connected condition, a microgrid is connected to the upstream distribution network which is considered to be an infinite bus with large power and energy capacities. During islanded condition, the control of the DG inverter operation is vital as DG inverters are the only components that can supply power generated from the DG units to the islanded microgrids.

Chapter 3 investigated the parallel operation of DG inverters in an AC microgrid, and it proposed a centralized control strategy with an MPC based local inverter control and a centralized control strategy in the EMS. The proposed MPC algorithm decomposed the DG inverter local control problem into two subproblems, i.e., steady-state subproblem and transient subproblem. By doing so, the MPC algorithm was able to track the reference control signals for the fast sampling LTI systems. A Kalman filter was introduced in the MPC algorithm to estimate the exogenous states that are needed for the optimization problem. Consequently, the Kalman filter could improve the power quality of the microgrid by enabling harmonic compensation for the harmonic currents of the load. In the various simulation test cases conducted in MATLAB/Simulink, the centralized control strategy proposed in the EMS was able to use the proposed MPC algorithm to accurately determine the power sharing between the different DG inverters during grid-connected and islanded conditions. Additionally, the impact of increasing the load in the distribution network on the PCC voltage was investigated in the proposed AC microgrid configuration. It was observed that the increments in real and reactive power load demand resulted in a significant drop in the PCC voltage. Moreover, the fault level due to the integration of DG units through the proposed microgrid was investigated as well. It verified that the increase in the fault currents would possibly result in using more expensive circuit breakers and distribution lines with larger capacities in comparison to a distribution network without DG units.

To further investigate the operation of DG inverters especially in an islanded AC microgrid with inverter-interfaced DG units that have no mechanical inertia, Chapter 4 
proposed a combined centralized and decentralized control strategy with a frequency droop based MPC DG inverter local control algorithm and a control strategy with a proposed load shedding scheme in the EMS. The frequency droop based MPC algorithm would allow most of the DG inverters to perform decentralized frequency droop control with one DG inverter controlling the frequency of the microgrid by receiving the reference control signal from the central controller. The proposed control strategy in the EMS would use the proposed frequency model, the frequency control method including the primary frequency control and the modified secondary frequency control, and the proposed load shedding scheme to maintain the operation of the DG inverters in an islanded AC microgrid. Several simulation test cases were conducted to verify the effectiveness of the proposed combined centralized and decentralized control strategy. The results proved that the DG inverters can operate effectively in an islanded AC microgrid with inverter-interfaced DG units.

Extending the study of the control of the inverter operation from AC microgrids to DC microgrids, Chapter 5 proposed a double-inverter configuration for a DC microgrid in order to harness the excessive wind energy in a poultry farm. The proposed configuration eliminated the need for voltage and frequency synchronization when integrating WTs, and it improved the system reliability in comparison to the single inverter configuration. In addition to the proposed microgrid configuration, an MPC algorithm was proposed for the CCM control and the VCM control of the two DG inverters. Through several simulation studies, the proposed DC microgrid configuration with the MPC control algorithm was able to maintain the operation of the microgrid under different situations. The results proved that the proposed configuration and the control of the inverter operation could offer increased flexibility and reliability to the operation of a DC microgrid.

This thesis also considered a few grid support functions of the DG inverters in gridconnected condition. Chapter 3 investigated the use of DG inverters for harmonic compensation. Chapter 4 analyzed the frequency control for frequency regulation. Chapters 4 and 5 studied the emergency power supply function when the microgrids are disconnected from the grid to operate in islanded condition. 


\subsection{Recommendations for Future Research}

Based on the limitations and the development of microgrid solutions, here list some of the areas and topics for future research works on inverter operation and microgrid solution:

1. As it can be observed that the proposed control strategies for inverter operations are only verified through simulation studies. It is not representative when it comes to hardware implementation. Therefore, the next step would be building the proposed AC and DC microgrids using hardware experiments or even in an experimental test bed. Control strategies and control algorithms proposed would need to be incorporated in the EMS central control and local programmable logic controllers to verify the performances through experimental results. Only when these control strategies are verified through real hardware setups, their feasibility and effectiveness can be proved. An example could be the high PV penetration in Singapore, which could cause potential stability problems. Singapore government plans to install rooftop PVs to $350 \mathrm{MW}$ by 2020 . Considering the intermittency of the PV and the loss of PV power generation due to weather conditions, the system voltage and frequency will be affected. To potentially overcome this problem, Energy Market Authority of Singapore launched a grant call in 2016 for testing a 2 MW battery test bed in a $22 \mathrm{KVA}$ substation. The main objective of this test bed is to understand the feasibility of deploying grid-level energy storage technologies to overcome the intermittency problem and to regulate the voltage and frequency.

2. Apart from grid-connected and islanded conditions, the transition period of the islanding process from grid-connected condition to islanded condition is worth investigating. Different control strategies are usually applied in grid-connected and islanded conditions. Therefore, during the transition period, due to the limitation resulted from the response time of sensors and power electronic devices, large voltage drop or frequency drop may occur in the microgrid as the DG inverters are still in the operating mode which is used in grid-connected condition. Especially during unexpected islanding process, the circuit breaker which links the microgrid with the upstream distribution network may open in 
a very short time to prevent the microgrid from collapse. This problem could be tackled in several different ways. One possible solution is to define a novel control strategy and algorithm for the grid-connected condition which can also sustain the operation of the microgrid during islanded condition. Additionally, as this thesis has only conducted analysis on applying the proposed control strategies in steady-state conditions, transient studies need to be carried out to verify different control strategies.

3. During the transition from grid-connected to isolated condition, other than the voltage and frequency drop problem, another critical issue is for DG inverters to maintain the voltage magnitude and phase with reference to that of the load voltage. Before the microgrid is islanded from the upstream distribution network, the DG inverters in the microgrid are working in CCM. They rely on the upstream distribution network to provide the voltage reference. When the circuit breaker that links the microgrid and the upstream distribution network opens, the DG inverters suddenly lose the voltage reference, and they are required to work in VCM to maintain the voltage. Therefore, how to maintain the magnitude and phase of the DG inverter output voltage with that of the load voltage is very crucial for the islanded microgrid. This problem would require further research to find the best control strategy to guarantee the islanding process is smooth.

4. Fault level study is very important for the design of protection system for the microgrid. In this thesis, Chapter 3 has demonstrated the increase of fault level in a microgrid. More detailed studies and experiments need to be conducted to address this issue in detail. Possible solutions could be proposed and verified to solve this issue in a microgrid with various kinds of DG units.

5. For a DC microgrid that uses multiple DG inverters to connect the DC bus to AC bus, coordination between DG inverters is very important. In this thesis, the double-inverter configuration is controlled by a centralized MPC algorithm for the coordination. However, in a more complicated situation with three and more DG inverters, a more advanced control algorithm can be developed to coordinate and control all the inverters, such as master and slave control algorithm, or decentralized control algorithm. This research would greatly benefit the operation of multiple DG inverters in a DC microgrid to improve its reliability. 


\section{REFERENCES}

[1] The BP statistical review of world energy 2016 [Online]. Available: https://www.bp.com/content/dam/bp/pdf/energy-economics/statistical-review2016/bp-statistical-review-of-world-energy-2016-full-report.pdf

[2] H. B. Puttgen, P. R. MacGregor, and F. C. Lambert, "Distributed generation: Semantic hype or the dawn of a new era?," IEEE Power and Energy Magazine, vol. 1, no. 1, pp. 22-29, 2003.

[3] P. Mahat, Z. Chen, B. Bak-Jensen, and C. L. Bak, "A Simple Adaptive Overcurrent Protection of Distribution Systems With Distributed Generation," IEEE Trans. Smart Grid, vol. 2, no. 3, pp. 428-437, 2011.

[4] T. Senjyu, Y. Miyazato, A. Yona, N. Urasaki, and T. Funabashi, "Optimal Distribution Voltage Control and Coordination With Distributed Generation," IEEE Trans. Power Delivery, vol. 23, no. 2, pp. 1236-1242, 2008.

[5] R. Williams, "Becquerel photovoltaic effect in binary compounds," The Journal of Chemical Physics, vol. 32, no. 5, pp. 1505-1514, 1960.

[6] Global market outlook for solar power 2015-2019 [Online]. Available: https://helapco.gr/pdf/Global_Market_Outlook_2015_-2019_lr_v23.pdf

[7] Snapshot of global PV 1992-2014. photovoltaic power systems programme [Online]. Available: http://www.iea-pvps.org/fileadmin/dam/public/report/ technical/PVPS_report_-_A_Snapshot_of_Global_PV_-_1992-2014.pdf

[8] H. Kanchev, D. Lu, F. Colas, V. Lazarov, and B. Francois, "Energy management and operational planning of a microgrid with a PV-based active generator for smart grid applications," IEEE Trans. Industrial Electronics, vol. 58, no. 10, pp. 4583-4592, Oct. 2011.

[9] C. A. Hill, M. C. Such, D. Chen, J. Gonzalez, and W. M. Grady, "Battery energy storage for enabling integration of distributed solar power generation," IEEE Trans. Smart Grid, vol. 3, no. 2, pp. 850-857, Jun. 2012.

[10] D. Rekioua and E. Matagne, "Modeling of solar irradiance and cells," in Optimization of Photovoltaic Power Systems: Springer, 2012, pp. 31-87.

[11] T. J. Price, "James Blyth-Britain's first modern wind power pioneer," Wind Engineering, vol. 29, no. 3, pp. 191-200, 2005.

[12] C. Meyer, M. Hoing, A. Peterson, and R. W. De Doncker, "Control and design of DC grids for offshore wind farms," IEEE Trans. Industry applications, vol. 43, no. 6, pp. 1475-1482, Nov. 2007.

[13] X. Ke, N. Lu, and C. Jin, "Control and size energy storage systems for managing energy imbalance of variable generation resources," IEEE Trans. Sustainable Energy, vol. 6, no. 1, pp. 70-78, Jan. 2015.

[14] V. Monteiro et al., "Assessment of a battery charger for electric vehicles with reactive power control," in Proc. 38th Annual Conference on IEEE Industrial Electronics Society (IECON), 2012, pp. 5142-5147.

[15] S. V. Papaefthymiou, E. G. Karamanou, S. A. Papathanassiou, and M. P. Papadopoulos, "A wind-hydro-pumped storage station leading to high RES penetration in the autonomous island system of Ikaria," IEEE Trans. Sustainable Energy, vol. 1, no. 3, pp. 163-172, Oct. 2010.

[16] H. Akagi and H. Sato, "Control and performance of a doubly-fed induction machine intended for a flywheel energy storage system," IEEE Trans. Power Electronics, vol. 17, no. 1, pp. 109-116, Jan. 2002. 
[17] K. Divya and J. Østergaard, "Battery energy storage technology for power systems-An overview," Electric Power Systems Research, vol. 79, no. 4, pp. 511-520, Apr. 2009.

[18] C. F. Lu, C. C. Liu, and C. J. Wu, "Dynamic modelling of battery energy storage system and application to power system stability," IEE ProceedingsGeneration, Transmission and Distribution, vol. 142, no. 4, pp. 429-435, Jul. 1995.

[19] X. Li, D. Hui, L. Wu, and X. Lai, "Control strategy of battery state of charge for wind/battery hybrid power system," in Proc. IEEE International Symposium on Industrial Electronics, 2010, pp. 2723-2726.

[20] X. Li, D. Hui, and X. Lai, "Battery energy storage station (BESS)-based smoothing control of photovoltaic (PV) and wind power generation fluctuations," IEEE Trans. Sustainable Energy, vol. 4, no. 2, pp. 464-473, Apr. 2013.

[21] M. S. Mahmoud and M. Fouad, Control and optimization of distributed generation systems. Springer, 2015.

[22] B. M. Weedy, B. J. Cory, N. Jenkins, J. Ekanayake, and G. Strbac, Electric Power Systems. John Wiley \& Sons, 2012.

[23] C. Wang and P. Li, "Development and challenges of distributed generation, the micro-grid and smart distribution system," Automation of Electric Power Systems, vol. 2, p. 004, Jan. 2010.

[24] J. P. Lopes, N. Hatziargyriou, J. Mutale, P. Djapic, and N. Jenkins, "Integrating distributed generation into electric power systems: a review of drivers, challenges and opportunities," Electric Power Systems Research, vol. 77, no. 9, pp. 1189-1203, Jul. 2007.

[25] W. Su and J. Wang, "Energy management systems in microgrid operations," The Electricity Journal, vol. 25, no. 8, pp. 45-60, Oct. 2012.

[26] N. Hatziargyriou, Microgrids: architectures and control. John Wiley \& Sons, 2013.

[27] About REIDS [Online]. Available: http://erian.ntu.edu.sg/REIDS/Pages /AboutREIDS.aspx

[28] E. Wood. US DOE channels over $\$ 10 \mathrm{M}$ to microgrids for grid R\&D [Online]. Available: https://microgridknowledge.com/microgrid-funding/

[29] C. Marnay, "A green prison: Santa Rita jail creeps towards zero net energy (ZNE)," Lawrence Berkeley National Laboratory, 2011.

[30] J. J. Justo, F. Mwasilu, J. Lee, and J.-W. Jung, "AC-microgrids versus DCmicrogrids with distributed energy resources: A review," Renewable and Sustainable Energy Reviews, vol. 24, pp. 387-405, 2013.

[31] J. Rocabert, A. Luna, F. Blaabjerg, and P. Rodriguez, "Control of power converters in ac microgrids," IEEE Trans. Power Electronics, vol. 27, no. 11, pp. 4734-4749, Nov. 2012.

[32] D. Fregosi et al., "A comparative study of dc and ac microgrids in commercial buildings across different climates and operating profiles," in Proc. IEEE First International Conference on DC Microgrids (ICDCM), 2015, pp. 159-164.

[33] Y. Li and J. Zheng, "A low-cost adaptive multi-mode digital control solution maximizing AC/DC power supply efficiency," in Proc. Applied Power Electronics Conference and Exposition (APEC), 2010, pp. 349-354.

[34] R. Majumder, "A hybrid microgrid with dc connection at back to back converters,” IEEE Trans. Smart Grid, vol. 5, no. 1, pp. 251-259, Jan. 2014. 
[35] L. $\mathrm{Xu}$ and D. Chen, "Control and operation of a dc microgrid with variable generation and energy storage," IEEE Trans. Power Delivery, vol. 26, no. 4, pp. 2513-2522, Oct. 2011.

[36] E. S. Hoff and L. E. Norum, "Inverter control for distributed generation," in Proc. Power Electronics and Motion Control Conference, 2006, pp. 544-549: IEEE.

[37] L. Hassaine, E. OLias, J. Quintero, and V. Salas, "Overview of power inverter topologies and control structures for grid connected photovoltaic systems," Renewable and Sustainable Energy Reviews, vol. 30, pp. 796-807, Feb. 2014.

[38] J. M. Guerrero, J. Matas, L. G. de Vicuna, M. Castilla, and J. Miret, "Decentralized control for parallel operation of distributed generation inverters using resistive output impedance," IEEE Trans. industrial electronics, vol. 54, no. 2, pp. 994-1004, Apr. 2007.

[39] J. C. Vasquez, J. M. Guerrero, A. Luna, P. Rodríguez, and R. Teodorescu, "Adaptive droop control applied to voltage-source inverters operating in gridconnected and islanded modes," IEEE Trans. Industrial Electronics, vol. 56, no. 10 , pp. 4088-4096, Oct. 2009.

[40] Southeast Energy Outlook 2015 [Online]. Available: https://www.iea.org/ publications/freepublications/publication/WEO2015_SouthEastAsia.pdf

[41] F. Resende, N. Gil, and J. Lopes, "Service restoration on distribution systems using Multi - MicroGrids,” European Trans. Electrical Power, vol. 21, no. 2, pp. 1327-1342, Mar. 2011.

[42] C. A. Hernandez-Aramburo, T. C. Green, and N. Mugniot, "Fuel consumption minimization of a microgrid," IEEE Trans. Industry Applications, vol. 41, no. 3, pp. 673-681, May 2005.

[43] W. Kühn, "Control and stability of power inverters feeding renewable power to weak ac grids with no or low mechanical inertia," in Proc. Power Systems Conference and Exposition, 2009, pp. 1-8.

[44] M. Czarick and J. Worley, "Wind turbines and tunnel fans," Poultry Housing Tips, vol. 22, no. 7, pp. 1-2, Jun. 2010.

[45] The poultry guide: environmentally control poultry farm ventilation systems for broiler, layer, breeders and top suppliers [Online]. Available: http://thepoultryguide.com/poultry-ventilation/

[46] Farm energy: energy efficient fans for poultry production [Online]. Available: http://farmenergy.exnet.iastate.edu

[47] G. Walker, "Evaluating MPPT converter topologies using a matlab PV model," Journal of Electrical \& Electronics Engineering, vol. 21, no. 1, pp. 49-56, Mar. 2001.

[48] R. F. Coelho, F. Concer, and D. C. Martins, "A study of the basic DC-DC converters applied in maximum power point tracking," in Proc. Brazilian Power Electronics Conference, 2009, pp. 673-678.

[49] F. Blaabjerg and Z. Chen, "Power electronics for modern wind turbines," Synthesis Lectures on Power Electronics, vol. 1, no. 1, pp. 1-68, Dec. 2005.

[50] R. Zamora and A. K. Srivastava, "Controls for microgrids with storage: Review, challenges, and research needs," Renewable and Sustainable Energy Reviews, vol. 14, no. 7, pp. 2009-2018, 9 Sept. 2010.

[51] J. A. P. Lopes, C. L. Moreira, and F. O. Resende, "Control strategies for microgrids black start and islanded operation," International Journal of Distributed Energy Resources, vol. 1, no. 3, pp. 241-261, 2005. 
[52] N. Hatziargyriou, A. Dimeas, and A. Tsikalakis, "Centralized and decentralized control of microgrids," International Journal of Distributed Energy Resources, vol. 1, no. 3, pp. 197-212, 2005.

[53] D. I. Brandao, T. Caldognetto, F. P. Marafão, M. G. Simões, J. A. Pomilio, and P. Tenti, "Centralized dontrol of distributed single-phase inverters arbitrarily connected to three-phase four-wire microgrids," IEEE Trans. Smart Grid, vol. 8, no. 1, pp. 437-446, Jan. 2017.

[54] M. M. A. Abdelaziz, M. F. Shaaban, H. E. Farag, and E. F. El-Saadany, "A multistage centralized control scheme for islanded microgrids with PEVs," IEEE Trans. Sustainable Energy, vol. 5, no. 3, pp. 927-937, Apr. 2014.

[55] L. I. Minchala-Avila, L. Garza-Castañón, Y. Zhang, and H. J. A. Ferrer, "Optimal energy management for stable operation of an islanded microgrid," IEEE Trans. Industrial Informatics, vol. 12, no. 4, pp. 1361-1370, Aug. 2016.

[56] C. Dou, M. Lv, T. Zhao, Y. Ji, and H. Li, "Decentralised coordinated control of microgrid based on multi-agent system," IET Generation, Transmission \& Distribution, vol. 9, no. 16, pp. 2474-2484, Nov. 2015.

[57] W. Liu, W. Gu, W. Sheng, X. Meng, Z. Wu, and W. Chen, "Decentralized multi-agent system-based cooperative frequency control for autonomous microgrids with communication constraints," IEEE Trans. Sustainable Energy, vol. 5, no. 2, pp. 446-456, Jan. 2014.

[58] A. Khorsandi, M. Ashourloo, and H. Mokhtari, "A decentralized control method for a low-voltage dc microgrid," IEEE Trans. Energy Conversion, vol. 29, no. 4, pp. 793-801, Jun. 2014.

[59] W. Shi, X. Xie, C. C. Chu, and R. Gadh, "Distributed optimal energy management in microgrids," IEEE Trans. Smart Grid, vol. 6, no. 3, pp. 11371146, May 2015.

[60] H. Cai and G. Hu, "Distributed power sharing control of grid-connected ac microgrid," in Proc. 55th Conference on Decision and Control (CDC), Dec. 2016, pp. 4290-4295.

[61] Y. Deng, Y. Tao, G. Chen, G. Li, and X. He, "Enhanced power flow control for grid-connected droop-controlled inverters with improved stability," IEEE Trans. Industrial Electronics, vol. PP, no. 99, pp. 1-10, Sept. 2016.

[62] A. A. M. Zin, A. Naderipour, M. H. Habibuddin, and J. M. Guerrero, "Harmonic currents compensator GCI at the microgrid," Electronics Letters, vol. 52, no. 20, pp. 1714-1715, Sept. 2016.

[63] X. Tang, X. Hu, N. Li, W. Deng, and G. Zhang, "A novel frequency and voltage control method for islanded microgrid based on multienergy storages," IEEE Trans. Smart Grid, vol. 7, no. 1, pp. 410-419, Dec. 2016.

[64] P. Sreekumar and V. Khadkikar, "Direct control of the inverter impedance to achieve controllable harmonic sharing in the islanded microgrid," IEEE Trans. Industrial Electronics, vol. 64, no. 1, pp. 827-837, Jan. 2017.

[65] C. L. Wadhwa, Electrical Power Systems. New Age International, 2005.

[66] Y. Li, D. M. Vilathgamuwa, and P. C. Loh, "Design, analysis, and real-time testing of a controller for multibus microgrid system," IEEE Trans. Power Electronics, vol. 19, no. 5, pp. 1195-1204, Sept. 2004.

[67] M. C. Chandorkar, D. M. Divan, and R. Adapa, "Control of parallel connected inverters in standalone ac supply systems," IEEE Trans. Industry Applications, vol. 29, no. 1, pp. 136-143, Jan. 1993.

[68] H. Mahmood, D. Michaelson, and J. Jiang, "Strategies for independent deployment and autonomous control of PV and battery units in islanded 
microgrids," IEEE Journal of Emerging and Selected Topics in Power Electronics, vol. 3, no. 3, pp. 742-755, Sept. 2015.

[69] M. J. Hossain, H. R. Pota, M. A. Mahmud, and M. Aldeen, "Robust control for power sharing in microgrids with low-inertia wind and PV generators," IEEE Trans. Sustainable Energy, vol. 6, no. 3, pp. 1067-1077, Jul. 2015.

[70] D. Wu, F. Tang, T. Dragicevic, J. C. Vasquez, and J. M. Guerrero, “A control architecture to coordinate renewable energy sources and energy storage systems in islanded microgrids," IEEE Trans. Smart Grid, vol. 6, no. 3, pp. 1156-1166, May 2015.

[71] J. Tian, Z. Liu, J. Shu, J. Liu, and J. Tang, "Base on the ultra-short term power prediction and feed-forward control of energy management for microgrid system applied in industrial park," IET Generation, Transmission \& Distribution, vol. 10, no. 9, pp. 2259-2266, Jun. 2016.

[72] A. Merabet, K. T. Ahmed, H. Ibrahim, R. Beguenane, and A. M. Y. M. Ghias, "Energy management and control system for laboratory scale microgrid based wind-PV-battery," IEEE Trans. Sustainable Energy, vol. 8, no. 1, pp. 145-154, Jan. 2017.

[73] T. Vandoorn, J. De Kooning, B. Meersman, and L. Vandevelde, "Review of primary control strategies for islanded microgrids with power-electronic interfaces," Renewable and Sustainable Energy Reviews, vol. 19, pp. 613-628, Mar. 2013.

[74] D. Chen, L. Xu, and L. Yao, "Dc voltage variation based autonomous control of dc microgrids," IEEE Trans. Power Delivery, vol. 28, no. 2, pp. 637-648, Apr. 2013.

[75] H. Frank and S. Ivner, "Thyristor-controlled shunt compensation in power networks," ASEA Journal, vol. 54, no. 5/6, pp. 121-127, 1981.

[76] J. J. Grainger and S. Civanlar, "Volt/Var control on distribution systems with lateral branches using shunt capacitors and voltage regulators part I: the overall problem," IEEE Trans. Power Apparatus and Systems, no. 11, pp. 3278-3283, Nov. 1985.

[77] C. E. Association and R. Mathur, Static compensators for reactive power control. Context Publications, 1984.

[78] V. Kekatos, G. Wang, A. J. Conejo, and G. B. Giannakis, "Stochastic reactive power management in microgrids with renewables," IEEE Trans. Power Systems, vol. 30, no. 6, pp. 3386-3395, Nov. 2015.

[79] R. Majumder, "Reactive power compensation in single-phase operation of microgrid," IEEE Trans. Industrial Electronics, vol. 60, no. 4, pp. 1403-1416, Apr. 2013.

[80] J. M. Guerrero, J. Matas, L. G. d. Vicuna, M. Castilla, and J. Miret, "Decentralized control for parallel operation of distributed generation inverters using resistive output impedance," IEEE Trans. Industrial Electronics, vol. 54, no. 2, pp. 994-1004, Apr. 2007.

[81] M. Savaghebi, A. Jalilian, J. C. Vasquez, and J. M. Guerrero, "Autonomous voltage unbalance compensation in an islanded droop-controlled microgrid," IEEE Trans. Industrial Electronics, vol. 60, no. 4, pp. 1390-1402, Apr. 2013.

[82] D. N. Zmood, D. G. Holmes, and G. H. Bode, "Frequency-domain analysis of three-phase linear current regulators," IEEE Trans. Industry Applications, vol. 37, no. 2, pp. 601-610, Apr. 2001. 
[83] F. Blaabjerg, R. Teodorescu, M. Liserre, and A. V. Timbus, "Overview of control and grid synchronization for distributed power generation systems," IEEE Trans. Industrial Electronics, vol. 53, no. 5, pp. 1398-1409, Oct. 2006.

[84] R. Teodorescu and M. Liserre, Grid converters for photovoltaic and wind power systems. John Wiley \& Sons, 2011.

[85] T. Erika and D. G. Holmes, "Grid current regulation of a three-phase voltage source inverter with an LCL input filter," IEEE Trans. Power Electronics, vol. 18, no. 3, pp. 888-895, May 2003.

[86] B. K. Bose, "An adaptive hysteresis-band current control technique of a voltage-fed PWM inverter for machine drive system," IEEE Trans. Industrial Electronics, vol. 37, no. 5, pp. 402-408, Oct. 1990.

[87] L. Malesani, P. Mattavelli, and P. Tomasin, "Improved constant-frequency hysteresis current control of VSI inverters with simple feedforward bandwidth prediction," IEEE Trans. Industry Applications, vol. 33, no. 5, pp. 1194-1202, Oct. 1997.

[88] P. Mattavelli, G. Spiazzi, and P. Tenti, "Predictive digital control of power factor preregulators with input voltage estimation using disturbance observers," IEEE Trans. Power Electronics, vol. 20, no. 1, pp. 140-147, Jan. 2005.

[89] G. Shen, D. Xu, L. Cao, and X. Zhu, "An improved control strategy for gridconnected voltage source inverters with an LCL filter," IEEE Trans. Power Electronics, vol. 23, no. 4, pp. 1899-1906, Jul. 2008.

[90] Y. Zhang, R. Wang, T. Zhang, Y. Liu, and B. Guo, "Model predictive controlbased operation management for a residential microgrid with considering forecast uncertainties and demand response strategies," IET Generation, Transmission \& Distribution, vol. 10, no. 10, pp. 2367-2378, Jul. 2016.

[91] J. Sachs and O. Sawodny, "A two-stage model predictive control strategy for economic diesel-PV-battery island microgrid operation in rural areas," IEEE Trans. Sustainable Energy, vol. 7, no. 3, pp. 903-913, Jul. 2016.

[92] A. Parisio, E. Rikos, and L. Glielmo, "A model predictive controlapproach to microgrid operation optimization," IEEE Trans. Control Systems Technology, vol. 22, no. 5, pp. 1813-1827, Sept. 2014.

[93] S. Kouro, P. Cortes, R. Vargas, U. Ammann, and J. Rodriguez, "Model predictive control-a simple and powerful method to control power converters," IEEE Trans. Industrial Electronics, vol. 56, no. 6, pp. 1826-1838, Jun. 2009.

[94] M. Tomlinson, H. d. T. Mouton, R. Kennel, and P. Stolze, "A fixed switching frequency scheme for finite-control-set model predictive control: concept and algorithm," IEEE Trans. Industrial Electronics, vol. 63, no. 12, pp. 7662-7670, Dec. 2016.

[95] N. Panten, N. Hoffmann, and F. W. Fuchs, "Finite control set model predictive current control for grid-connected voltage-source converters with LCL filters: a study based on different state feedbacks," IEEE Trans. Power Electronics, vol. 31, no. 7, pp. 5189-5200, Jul. 2016.

[96] C. Xia, T. Liu, T. Shi, and Z. Song, "A simplified finite-control-set modelpredictive control for power converters," IEEE Trans. Industrial Informatics, vol. 10, no. 2, pp. 991-1002, May 2014.

[97] L. Guo et al., "Energy management system for stand-alone wind-powereddesalination microgrid," IEEE Trans. Smart Grid, vol. 7, no. 2, pp. 1079-1087, Mar. 2016.

[98] H. Mahmood, D. Michaelson, and J. Jiang, "A power management strategy for PV/battery hybrid systems in islanded microgrids," IEEE Journal of Emerging 
and Selected Topics in Power Electronics, vol. 2, no. 4, pp. 870-882, Dec. 2014.

[99] S. Arnborg, G. Andersson, D. J. Hill, and I. A. Hiskens, "On undervoltage load shedding in power systems," International Journal of Electrical Power \& Energy Systems, vol. 19, no. 2, pp. 141-149, Feb. 1997.

[100] R. Faranda, A. Pievatolo, and E. Tironi, "Load shedding: a new proposal," IEEE Trans. Power Systems, vol. 22, no. 4, pp. 2086-2093, Nov. 2007.

[101] C. W. Taylor, "Concepts of undervoltage load shedding for voltage stability," IEEE Trans. Power Delivery, vol. 7, no. 2, pp. 480-488, Apr. 1992.

[102] D. Kottick and O. Or, "Neural-networks for predicting the operation of an under-frequency load shedding system," IEEE Trans. Power Systems, vol. 11, no. 3, pp. 1350-1358, Aug. 1996.

[103] J. A. Laghari, H. Mokhlis, M. Karimi, A. H. A. Bakar, and H. Mohamad, "A new under-frequency load shedding technique based on combination of fixed and random priority of loads for smart grid applications," IEEE Trans. Power Systems, vol. 30, no. 5, pp. 2507-2515, Sept. 2015.

[104] C. P. Reddy, S. Chakrabarti, and S. C. Srivastava, "A sensitivity-based method for under-frequency load-shedding," IEEE Trans. Power Systems, vol. 29, no. 2, pp. 984-985, Mar. 2014.

[105] H. Gao, Y. Chen, Y. Xu, and C. C. Liu, "Dynamic load shedding for an islanded microgrid with limited generation resources," IET Generation, Transmission \& Distribution, vol. 10, no. 12, pp. 2953-2961, Sept. 2016.

[106] Y. Y. Hong, M. C. Hsiao, Y. R. Chang, Y. D. Lee, and H. C. Huang, "Multiscenario underfrequency load shedding in amicrogrid consisting of intermittent renewables," IEEE Trans. Power Delivery, vol. 28, no. 3, pp. 1610-1617, Jul. 2013.

[107] M. Karimi, P. Wall, H. Mokhlis, and V. Terzija, "A new centralized adaptive under-frequency load shedding controller for microgrids based on a distribution state estimator," IEEE Trans. Power Delivery, vol. PP, no. 99, pp. 1-1, Jul. 2016.

[108] A. B. Mogstad, M. Molinas, P. K. Olsen, and R. Nilsen, "A power conversion system for offshore wind parks," in Proc. 34th Annual Conference of IEEE Industrial Electronics, Nov. 2008, pp. 2106-2112.

[109] D. Jovcic, "Offshore wind farm with a series multiterminal CSI HVDC," Electric Power Systems Research, vol. 78, no. 4, pp. 747-755, Apr. 2008.

[110] J. Chi, P. C. Loh, W. Peng, M. Yang, and F. Blaabjerg, "Autonomous operation of hybrid AC-DC microgrids," in Proc. IEEE International Conference on Sustainable Energy Technologies (ICSET), Dec. 2010, pp. 1-7.

[111] K. T. Tan, X. Y. Peng, P. L. So, Y. C. Chu, and M. Z. Q. Chen, "Centralized control for parallel operation of distributed generation inverters in microgrids," IEEE Trans. Smart Grid, vol. 3, no. 4, pp. 1977-1987, Dec. 2012.

[112] M. Charkhgard and M. Farrokhi, "State-of-charge estimation for lithium-ion batteries using neural networks and EKF," IEEE Trans. Industrial Electronics, vol. 57, no. 12, pp. 4178-4187, Dec. 2010.

[113] M. Coleman, C. K. Lee, C. Zhu, and W. G. Hurley, "State-of-charge determination from EMF voltage estimation: Using impedance, terminal voltage, and current for lead-acid and lithium-ion batteries," IEEE Trans. Industrial Electronics, vol. 54, no. 5, pp. 2550-2557, Oct. 2007. 
[114] W. Shen, C. Chan, E. W. Lo, and K. Chau, "Adaptive neuro-fuzzy modeling of battery residual capacity for electric vehicles," IEEE Trans. Industrial Electronics, vol. 49, no. 3, pp. 677-684, Jun. 2002.

[115] C. L. Chen, Y. Wang, J. S. Lai, Y. S. Lee, and D. Martin, "Design of parallel inverters for smooth mode transfer microgrid applications," IEEE Trans. Power Electronics, vol. 25, no. 1, pp. 6-15, Jan. 2010.

[116] A. Ghosh and G. Ledwich, Power quality enhancement using custom power devices. Springer Science \& Business Media, 2012.

[117] J. A. P. Lopes, C. L. Moreira, and A. G. Madureira, "Defining control strategies for microgrids islanded operation," IEEE Trans. Power Systems, vol. 21, no. 2, pp. 916-924, May 2006.

[118] K.-S. Low and R. Cao, "Model predictive control of parallel-connected inverters for uninterruptible power supplies," IEEE Trans. Industrial Electronics, vol. 55, no. 8, pp. 2884-2893, Aug. 2008.

[119] J. Mattingley, Y. Wang, and S. Boyd, "Receding horizon control," IEEE Control Systems, vol. 31, no. 3, pp. 52-65, Jun. 2011.

[120] K. H. Kwan, Y. C. Chu, and P. L. So, "Model-Based Ho Control of a Unified Power Quality Conditioner," IEEE Trans. Industrial Electronics, vol. 56, no. 7, pp. 2493-2504, Jul. 2009.

[121] C. Y. Teo, Principles and Design of Low Voltage Systems. Singapore: Byte Power Publications, 2015.

[122] A. Tuladhar, H. Jin, T. Unger, and K. Mauch, "Parallel operation of single phase inverter modules with no control interconnections," in Proc. Applied Power Electronics Conference and Exposition, 1997, pp. 94-100.

[123] C. L. Masters, "Voltage rise: the big issue when connecting embedded generation to long $11 \mathrm{kV}$ overhead lines," Power Engineering Journal, vol. 16, no. 1, pp. 5-12, Feb. 2002.

[124] N. Jenkins, N. Jenkins, J. Ekanayake, and G. Strbac, Distributed Generation. Institution of Engineering and Technology, 2010.

[125] X. Y. Peng and P. L. So, "Operation and control of distributed generation inverters in an islanded microgrid," submitted to IEEE Trans. Smart Grid, Jul. 2017.

[126] F. J. Lin and H. M. Su, "A high-performance induction motor drive with online rotor time-constant estimation," IEEE Trans. Energy Conversion, vol. 12, no. 4, pp. 297-303, Dec. 1997.

[127] P. M. Ashton, C. S. Saunders, G. A. Taylor, A. M. Carter, and M. E. Bradley, "Inertia estimation of the GB power system using synchrophasor measurements," IEEE Trans. Power Systems, vol. 30, no. 2, pp. 701-709, Mar. 2015.

[128] P. Wall and V. Terzija, "Simultaneous estimation of the time of disturbance and inertia in power systems," IEEE Trans. Power Delivery, vol. 29, no. 4, pp. 2018-2031, Aug. 2014.

[129] P. Cortes, G. Ortiz, J. I. Yuz, J. Rodriguez, S. Vazquez, and L. G. Franquelo, "Model predictive control of an inverter with output LC filter for UPS applications," IEEE Trans. Industrial Electronics, vol. 56, no. 6, pp. 18751883, 2009.

[130] M. Roozbehani, M. A. Dahleh, and S. K. Mitter, "Volatility of power grids under real-time pricing," IEEE Trans. Power Systems, vol. 27, no. 4, pp. 19261940, Nov. 2012. 
[131] D. T. Nguyen, M. Negnevitsky, and M. de Groot, "Modeling load recovery impact for demand response applications," IEEE Trans. Power Systems, vol. 28, no. 2, pp. 1216-1225, May 2013.

[132] Energy Market Authority of Singapore [Online]. Available: http://www.ema.gov.sg/

[133] K. T. Tan, B. Sivaneasan, X. Y. Peng, and P. L. So, "Control and operation of a dc grid-based wind power generation system in a microgrid," IEEE Trans. Energy Conversion, vol. 31, no. 2, pp. 496-505, Jun. 2016.

[134] N. Mendis, K. M. Muttaqi, S. Sayeef, and S. Perera, "Standalone operation of wind turbine-based variable speed generators with maximum power extraction capability," IEEE Trans. Energy Conversion, vol. 27, no. 4, pp. 822-834, Dec. 2012.

[135] S. X. Chen, H. B. Gooi, and M. Wang, "Sizing of energy storage for microgrids," IEEE Trans. Smart Grid, vol. 3, no. 1, pp. 142-151, Mar. 2012.

[136] T. Dragičević, J. M. Guerrero, J. C. Vasquez, and D. Škrlec, "Supervisory control of an adaptive-droop regulated DC microgrid with battery management capability," IEEE Trans. Power Electronics, vol. 29, no. 2, pp. 695-706, Feb. 2014.

[137] T. Kato, K. Tamura, and T. Matsuyama, "Adaptive storage battery management based on the energy on demand protocol," in Proc. IEEE Third International Conference on Smart Grid Communications (SmartGridComm), 2012, pp. 43-48.

[138] O. Gomis-Bellmunt, A. Junyent-Ferré, A. Sumper, and J. Bergas-Jané, "Control of a wind farm based on synchronous generators with a central HVDC-VSC converter," IEEE Trans. Power Systems, vol. 26, no. 3, pp. 16321640, Aug. 2011.

[139] W. Lu and B. T. Ooi, "Optimal acquisition and aggregation of offshore wind power by multiterminal voltage-source HVDC," IEEE Trans. Power Delivery, vol. 18, no. 1, pp. 201-206, Jan. 2003.

[140] L. Wang, Model predictive control system design and implementation using $M A T L A B \circledR$. Springer Science \& Business Media, 2009. 


\section{AUTHOR'S PUBLICATIONS}

\section{Journal Publications}

[1] K. T. Tan, X. Y. Peng, P. L. So, Y. C. Chu, and M. Z. Q. Chen, "Centralized control for parallel operation of distributed generation inverters in microgrids," IEEE Trans. Smart Grid, vol. 3, no. 4, pp. 1977-1987, Dec. 2012.

[2] K. T. Tan, B. Sivaneasan, X. Y. Peng, and P. L. So, "Control and operation of a dc grid based wind power generation system in a microgrid," IEEE Trans. Energy Conversion, vol. 31, no. 2, pp. 496-505, Jun. 2016.

[3] Y. Wang, K. T. Tan, X. Y. Peng, and P. L. So, "Coordinated control of distributed energy storage systems for voltage regulation in distribution networks," IEEE Trans. Power Delivery, vol. 31, no. 3, pp. 1132-1141, Jun. 2016.

[4] B. F. Wang, V. Kanamarlapudi, L. Xian, X. Y. Peng, K. T. Tan, and P. L. So, "Model predictive voltage control for single-inductor multiple-output DC-DC converter with reduced cross regulation," IEEE Trans. Industrial Electronics, vol. 63, no. 7, pp. 4187-4197, Jul. 2016.

[5] X. Y. Peng and P. L. So, "Operation and control of distributed generation inverters in an islanded microgrid," submitted to IEEE Trans. Smart Grid, Jul. 2017. (under review)

\section{Conference Publications}

[1] X. Y. Peng, P. L. So, and K. T. Tan, "An analysis on system frequency of a smart grid network with large-scale PV power generation," in Proc. IEEE PES Asia-Pacific Power and Energy Engineering Conference (APPEEC), Hong Kong, Dec. 2014, pp. 1-6. 